\title{
Pseudo-helicale und helicale Primärstrukturen aus spiroanellierten vier- und fünfgliedrigen Ringen: Synthesen und chiroptische Eigenschaften
}

\author{
Dissertation \\ Zur Erlangung des Doktorgrades \\ der Mathematisch-Naturwissenschaftlichen Fakultäten \\ der Georg-August-Universität Göttingen
}

vorgelegt von

Tien Widjaja

aus Surabaya, Indonesien

Göttingen 2005 
D 7

Referent: Prof. Dr. L. Fitjer

Korreferent: Prof. Dr. A. Zeeck

Tag der mündlichen Prüfung: 03.11.2005 
Die vorliegende Arbeit wurde unter der Leitung von Herrn Prof. Dr. L. Fitjer in der Zeit von März 2003 bis September 2005 am Organisch-Chemischen Institut der Georg-August Universität zu Göttingen durchgeführt.

An dieser Stelle möchte ich Herrn Prof. Dr. L. Fitjer für die Anregung zu diesem Thema, sein stetes Interesse am Fortgang der Arbeit, die ausgezeichneten Arbeitsbedingungen und zahlreiche fördernde Diskussionen danken. 
Meinen Eltern 


\section{INHALTSVERZEICHNIS}

\section{EINLEITUNG}

1 Versuche zur Cycloaddition von Keteniminiumsalzen an 1-Methylenspiro[3.3]heptan (21) und 1-Methylenspiro[4.4]nonan (25): Synthese von (5R*)-Trispiro[3.0.0.4.2.2]tetradecan [(5R*)-13)] und (5R*)-Trispiro[3.0.0.4.3.2]pentadecan $\left.\left[\left(5 R^{*}\right)-14\right)\right]$

1.1 Synthese der Vorprodukte

1.2 Versuche zur Cycloaddition von Keteniminiumsalzen an Methylenspiroalkane

1.3 Synthese von (5R*)-Trispiro[3.0.0.4.2.2]tetradecan [(5R*)-13] und (5R*)Trispiro[3.0.0.4.3.2]pentadecan [(5R*)-14]

2 Racemattrennung von (5R*)-Trispiro[3.0.0.4.2.2]tetradecan-11-on [(5R*)-24] durch 18 enantioselektive Reduktion: Synthese von (5R)-Trispiro[3.0.0.4.2.2]tetradecan [(5R)-13] und (5S)-Trispiro[3.0.0.4.3.2]pentadecan [(5S)-14]

2.1 Racemattrennung von (5R*)-Trispiro[3.0.0.4.2.2]tetradecan-11-on [(5R*)- 18 24]

2.2 Synthese von (5R)-Trispiro[3.0.0.4.2.2]tetradecan [(5R)-13] und (5S)-Trispiro[3.0.0.4.3.2]pentadecan [(5S)-14]

3 Synthesen von (5R*)-Trispiro[3.0.0.4.3.3]hexadecan [(5R*)-15] und rac-Trispiro[4.0.0.4.3.3]heptadecan (rac-8)

3.1 Synthesen über Dispiro[4.0.4.3]tridecan-1-on (30)

3.1.1 Ein neuer Zugang zu Dispiro[4.0.4.3]tridecan-1-on (30): Synthese und Umlagerung von Dispiro[3.1.4.3]tridecan-5-on (31)

3.1.2 Versuchte Cycloaddition von Dichlorketen an 1-Methylendispiro25 [3.0.4.3]tridecan (33) 
3.1.3 Sequentielle Transformation von 30 in $\left(4 R^{*}, 5 S^{*}\right)$-Trispiro-

[3.0.0.4.3.3]hexadecan-1-on [( $\left.\left.4 R^{*}, 5 S^{*}\right)-43\right]$ über $\left(1 S^{*}, 5 S^{*}\right)-1-(1-$ Ethoxycyclopropyl)dispiro[4.0.4.3]tridecan-1-ol [( $\left.\left.1 S^{*}, 5 S^{*}\right)-42\right]$ : Synthese von $\left(5 S^{*}\right)-\mathbf{1 5}$

3.1.4 Ringerweiterung von $\left(4 R^{*}, 5 S^{*}\right)-43$ über 1-Oxatetraspiro30 [2.0.0.0.4.3.3.2]octadecan [(3S*,4R*,5S*)-46]: Synthese von rac8

3.2 Synthesen von rac-15 und rac-8 über Dispiro[3.0.4.3]dodecan-1-on (51)

3.2.1 Synthese von $\mathbf{5 1}$

3.2.2 Sequentielle Transformation von $\left(4 S^{*}\right)-51$ in $\left(6 S^{*}, 7 S^{*}, 8 R^{*}\right)-8$ Brom-trispiro[4.0.0.4.3.3] heptadecan-12-on [(6S*,7S*,8R*)-54] und $\left(1 R^{*}, 4 S^{*}, 5 S^{*}\right)$-1-Brom-trispiro[3.0.0.4.3.3]hexadecan-16-on $\left[\left(1 R^{*}, 4 S^{*}, 5 S^{*}\right)-56\right]$ über ein Cyclopentenyl- bzw. Cyclobutenylcyclobutanol: verkürzte Synthese von rac-8

3.2.3 Sequentielle Transformation von (4S*)-51 in Trispiro-

[3.0.0.4.3.3]hexadecan-16-on (57) über ein $\beta$-Hydroxyselenid: verkürzte Synthese von (5S*)-15 $N, S$-Dimethyl-S-phenylsulfoximin und (-)-Diisopinocampheylchloroboran: Synthese von (5R)-Trispiro[3.0.0.4.3.3]hexadecan [(5R)-15] und $(P)$-Trispiro[4.0.0.4.3.3]heptadecan $[(P)-8]$

4.1 Racemattrennung von (4S*)-Dispiro[3.0.4.3]dodecan-1-on [(4S*)-51] mit 40 (+)-(S)-N,S-Dimethyl-S-phenylsulfoximin

4.2 Racemattrennung von (4S*)-Dispiro[3.0.4.3]dodecan-1-on [(4S*)-51] mit (-)-Diisopinocampheylchloroboran

4.3 Synthese von (5R)-Trispiro[3.0.0.4.3.3]hexadecan [(5R)-15] und $(P)$-Trispiro[4.0.0.4.3.3]heptadecan $[(P)-8]$

5 Kristallstrukturen von (1S,5'S,11'S)-(+)-79, $\left(4 R^{*}, 5 S^{*}\right)-\mathbf{9 8},\left(6 S^{*}, 7 S^{*}, 8 R^{*}\right)-\mathbf{5 4}$, $(\mathrm{SS}, 1 R, 4 R)-(+)-\mathbf{5 9},(\mathrm{S} S, 1 S, 4 S)-(+)-\mathbf{6 1}$ und $(1 S, 4 R)-(+)-\mathbf{1 0 4}$ 
ZUSAMMENFASSUNG

EXPERIMENTELLER TEIL

Allgemeine Angaben 56

Arbeitsvorschriften

SPEKTRENANHANG

IR-Spektren

${ }^{1} \mathrm{H}-\mathrm{NMR}-$ Spektren

105

${ }^{13}$ C-NMR-Spektren

ABBILDUNGSVERZEICHNIS

LITERATURVERZEICHNIS 


\section{EINLEITUNG}

Die interessante Frage, ob und in welchem Ausmaß die chiroptischen Eigenschaften helicaler Kohlenwasserstoffe aus spiroanellierten Ringen durch statische Parameter, wie Identitätsperiode, Ganghöhe und Länge der Helix, und/oder durch dynamische Phänomene, wie konformationelle Gleichgewichte beeinflusst wird, sollte am besten experimentell in Verbindung mit einer adäquaten theoretischen Behandlung beantwortbar sein. Zu diesem Zweck sollten helicale Strukturen aus Ringen unterschiedlicher Ringgröße, sowie, für fünfgliedrige und größere Ringe, mit unterschiedlicher Stellung der Spirozentren untersucht werden.

\section{Schema 1}

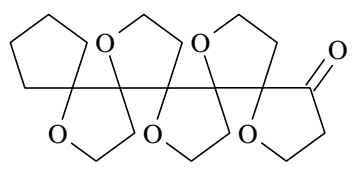

1

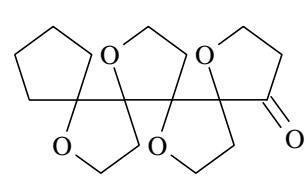

2

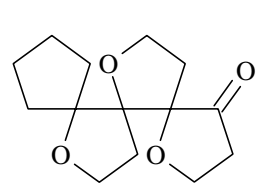

4

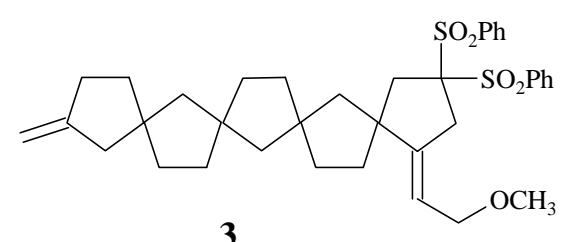

3

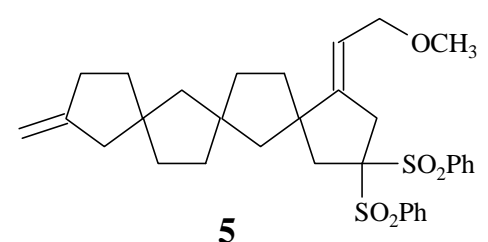

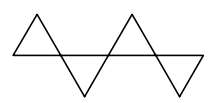

6

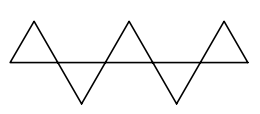

10

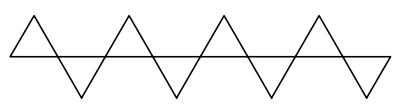

12

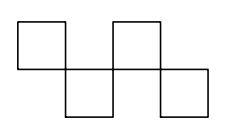

7

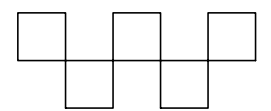

11

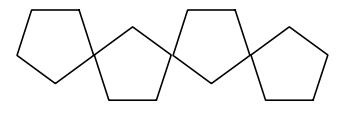

9

Wie am Beispiel der Minimalstrukturen helicaler Kohlenwasserstoffe aus spiroanellierten drei- (6), vier- (7) und fünfgliedrigen Ringen $(\mathbf{8}, \mathbf{9})$ gezeigt, gibt es für solche aus dreiund viergliedrigen Ringen lediglich eine, für solche aus fünfgliedrigen Ringen dagegen zwei 
Primärstrukturen (Schema 1). Von den aus Cyclopropanen aufgebauten Helices existieren Synthesen für $(P)$ - und $(M)-\mathbf{6},{ }^{1,2}(P)$ - und $(M)-\mathbf{1 0},{ }^{2}$ und $(P)$ - und $(M)-12^{3}$ aus dem Arbeitskreis de Meijere, von den aus Cyclobutanen aufgebauten Helices Synthesen für $(M)-7^{4}$, und $(P)$ und $(M)-\mathbf{1 1}^{5}$ aus unserem Arbeitskreis. Die aus 1,2- bzw. 1,3-spiroanellierten Cyclopentanen aufgebauten Helices 8 und 9 sind unbekannt. Bei den 1980 von Magnus ${ }^{6}$ dargestellten Polyethern 1, 2 und 3 ([3]-, [4]- und [5]-Helixan) handelt es sich um Heteroanaloga von 8, bei den 1991 von Trost ${ }^{7,8}$ synthetisierten Polyspiranen 3 und $\mathbf{5}$ um substituierte Analoga von 9. Ob $\mathbf{3}$ tatsächlich eine helicale Partialstruktur besitzt, ist offen.

Interessantester Befund der Arbeiten über [ $\sigma]$-Helices aus Cyclopropanen ${ }^{1-3}$ ist die hohe spezifische Drehung von $(M)-6\left([\alpha]_{D}{ }^{20}=-193\right.$ (c 1.18, $\left.\mathrm{CHCl}_{3}\right)$ ), sowie deren zweimalige Verdopplung beim Übergang zu $(M)-\mathbf{1 0}\left([\alpha]_{\mathrm{D}}{ }^{20}=-381\left(\mathrm{c} 1.2, \mathrm{CHCl}_{3}\right)\right)$ und $(M)-\mathbf{1 2}\left([\alpha]_{\mathrm{D}}{ }^{20}=-\right.$ 891 (с 1.01, $\left.\mathrm{CHCl}_{3}\right)$ ). Im Gegensatz dazu ist die spezifische Drehung von [ $\left.\sigma\right]$-Helices aus Cyclobutanen $^{4,5}$ bei $(M)-7\left([\alpha]_{\mathrm{D}}{ }^{20}=-63\right.$ (c 1.09, $\left.\mathrm{CHCl}_{3}\right)$ ) von vornherein klein und nimmt beim Übergang zu $(M)-\mathbf{1 1}\left([\alpha]_{\mathrm{D}}{ }^{20}=-24\left(\mathrm{c} 1.17, \mathrm{CHCl}_{3}\right)\right.$ ) weiter ab. Hierin kommt zum Ausdruck, dass in geometrisch fixierten Systemen wie 6, 10 und 12 ausschließlich statische Parameter über den Drehwert entscheiden, während in flexiblen Systemen wie $\mathbf{7}$ und $\mathbf{1 1}$ der Drehwert aus einer Boltzmann-gewichteten Summation der Drehwerte unterschiedlicher Konformationen resultiert. Diese wiederum können nicht experimentell, wohl aber über ab initio-Rechnungen erfasst werden. Natürlich ist der Aufwand wegen der Notwendigkeit, einen genügend großen Satz potentiell niederenergetischer Konformerer zu optimieren und deren optische Drehung zu berechnen, relativ hoch. Gleichwohl gibt es keinen anderen Weg, den Einfluss unterschiedlicher Konformationen auf den Drehwert zu erfassen.

\section{Schema 2}

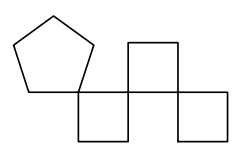

13

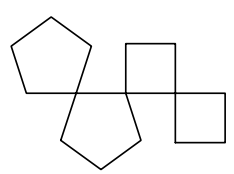

14

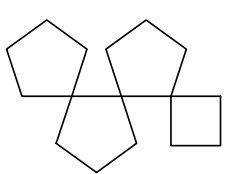

15

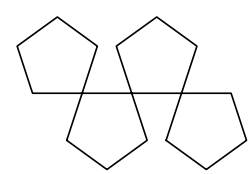

8

Rechnungen in der Abteilung von Herrn Prof. Grimme am Institut für Theoretische Chemie der Universität Münster haben gezeigt, dass das chiroptische Verhalten von $\mathbf{7}$ und $\mathbf{1 1}$ mit ab initio-Methoden modelliert werden kann. ${ }^{9}$ Ziel der vorliegenden Arbeit war daher, die Zahl der Freiheitsgrade für konformationelle Umwandlungen nochmals zu erhöhen und jeden der vier Vierringe in 7 schrittweise durch einen Fünfring zu ersetzen (Schema 2). Die resultierenden pseudohelicalen Trispirane 13, 14 und 15 sowie das helicale Trispiran 8 sollten racemisch und optisch rein synthetisiert und die spezifischen Drehungen bei verschiedenen Wellenlängen gemessen und für weitere Rechnungen zur Verfügung gestellt werden. Diese Rechnungen sollten auch Helices $\mathbf{7}$ und $\mathbf{8}$ mit über Geometrierestriktionen planarisierten Vier- 
bzw. Fünfringen mit einbeziehen, um so den Einfluss statischer Parameter wie Identitätsperiode, Ganghöhe und Länge der Helix getrennt von dem konformationeller Gleichgewichte zu erfassen.

Bezüglich der zu realisierenden Synthesen sei erwähnt, dass in früheren Arbeiten unseres Arbeitskreises ${ }^{10}$ versucht wurde, acyclisch 1,1-verknüpfte Polycyclobutane $\mathbf{1 6}$ mit $\mathbf{n} \geq$ 5 darzustellen und das hohe thermodynamische Gefälle von $\mathrm{C}_{4}-\mathrm{C}_{5}$-Ringerweiterungen ${ }^{11,12} \mathrm{zu}$ einer Umlagerung geeignet substituierter Derivate in helicale Polyspirane des Typs 8 zu nutzen (Schema 3). Modellrechnungen mit MMP2 ${ }^{13}$ an dem Septicyclobutan $\mathbf{1 7}\left(\mathrm{R}^{1}=\mathrm{R}^{2}=\right.$ $\mathrm{CH}_{3}$ ) hatten nämlich ergeben, dass diese Verbindung eine helicale Konformation $\mathbf{B}$ gegenüber einer Zick-Zack-Konformation A bevorzugt $\left(\Delta \Delta \mathrm{H}_{\mathrm{f}}{ }^{\circ}=71.8 \mathrm{kcal} / \mathrm{mol}\right)$. Es schien daher möglich, dass ein geeignet funktionalisiertes Derivat (z.B. 17, $\mathrm{R}^{1}=\mathrm{CH}_{2} \mathrm{OH}, \mathrm{R}^{2}=\mathrm{CH}_{3}$ ) konformativ kontrolliert in ein Hexaspiran 18 mit einer helicalen Primärstruktur 19 umlagern würde. Leider gelang der Aufbau acyclisch 1,1-verknüpfter Polycyclobutane 16 mit $\mathrm{n} \geq 5$ bisher nicht. ${ }^{10}$ Der Ansatz scheint nach wie vor attraktiv, sollte allerdings in der vorliegenden Arbeit nicht weiter verfolgt werden. Vielmehr war geplant, die Versuche zur Synthese von 13, 14, 15 und 8 methodisch an den erfolgreichen Synthesen von $7^{4,5} \mathrm{zu}$ orientieren.

\section{Schema 3}

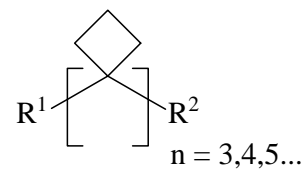

16<smiles>C=CC(C)C(C)C(C)C(C)C(C)C(C)CC</smiles>

A

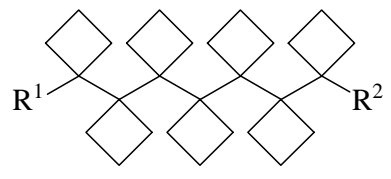

17

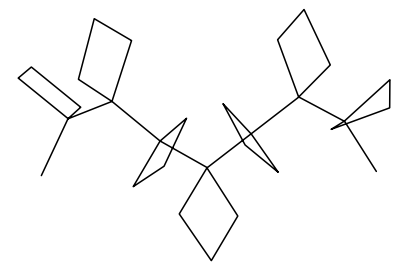

B

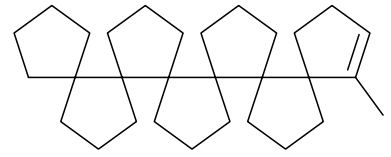

18

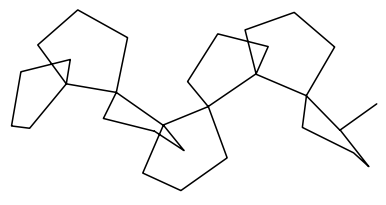

19

Schlüsselschritt der kürzeren ${ }^{5}$ von zwei Synthesen von $7^{4,5}$ ist eine [2+2]Cycloaddition eines aus dem Amid 20 generierten Keteniminiumsalzes an das Methylenspiroheptan 21 unter Bildung des Trispiroketons 22 (Schema 4). Die Racemattrennung gelang durch enantioselektive Reduktion wahlweise enzymatisch mit Bäckerhefe ${ }^{14}$ oder chemisch mit (1R)-(-)-B-Chlordiisopinocampheylboran $^{15}$ [(-)-DIP-Chlorid] und Rückoxidation mit Pyridiniumchlorochromat. ${ }^{16}$ Wolff-Kishner Reduktion ${ }^{17,18}$ komplettierte die Synthesen 7 und (M)-7. 


\section{Schema 4}

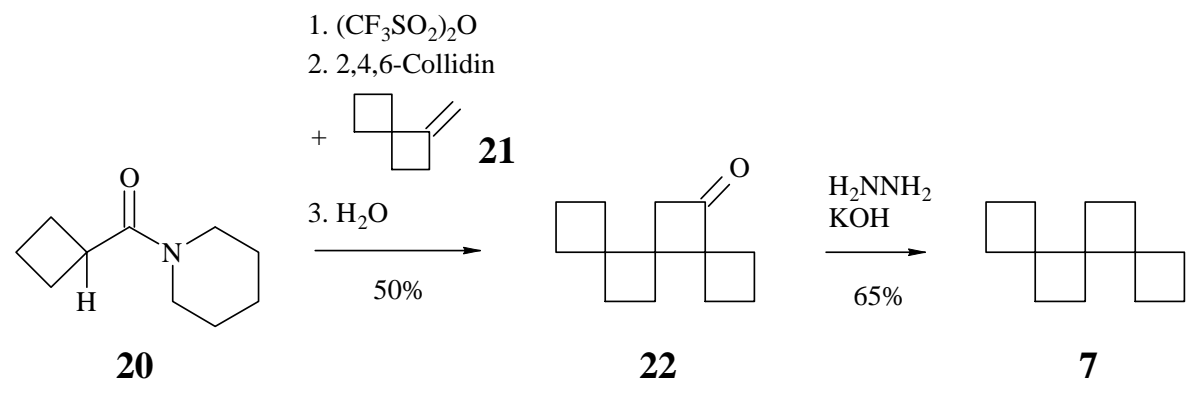

Damit war klar, dass zumindest eine [2+2]Cycloaddition eines von dem Amid 23 abgeleiteten Keteniminiumsalzes an $\mathbf{2 1}$ gute Erfolgsaussichten besaß. Darüber hinaus schien jedoch möglich, dass sich beide Keteniminiumsalze auch an das Methylenspirononan $25^{19-22}$ addieren würden. Im günstigsten Fall wären damit alle Syntheseziele erreichbar gewesen: 13 über 24, 14 über 24 und/oder 26, 15 über 26, und 8 über 27 (Schema 5). Die zur Komplettierung notwendigen Reaktionen schienen unproblematisch und eine Racemattrennung auf der Stufe der Ketone möglich.

\section{Schema 5}

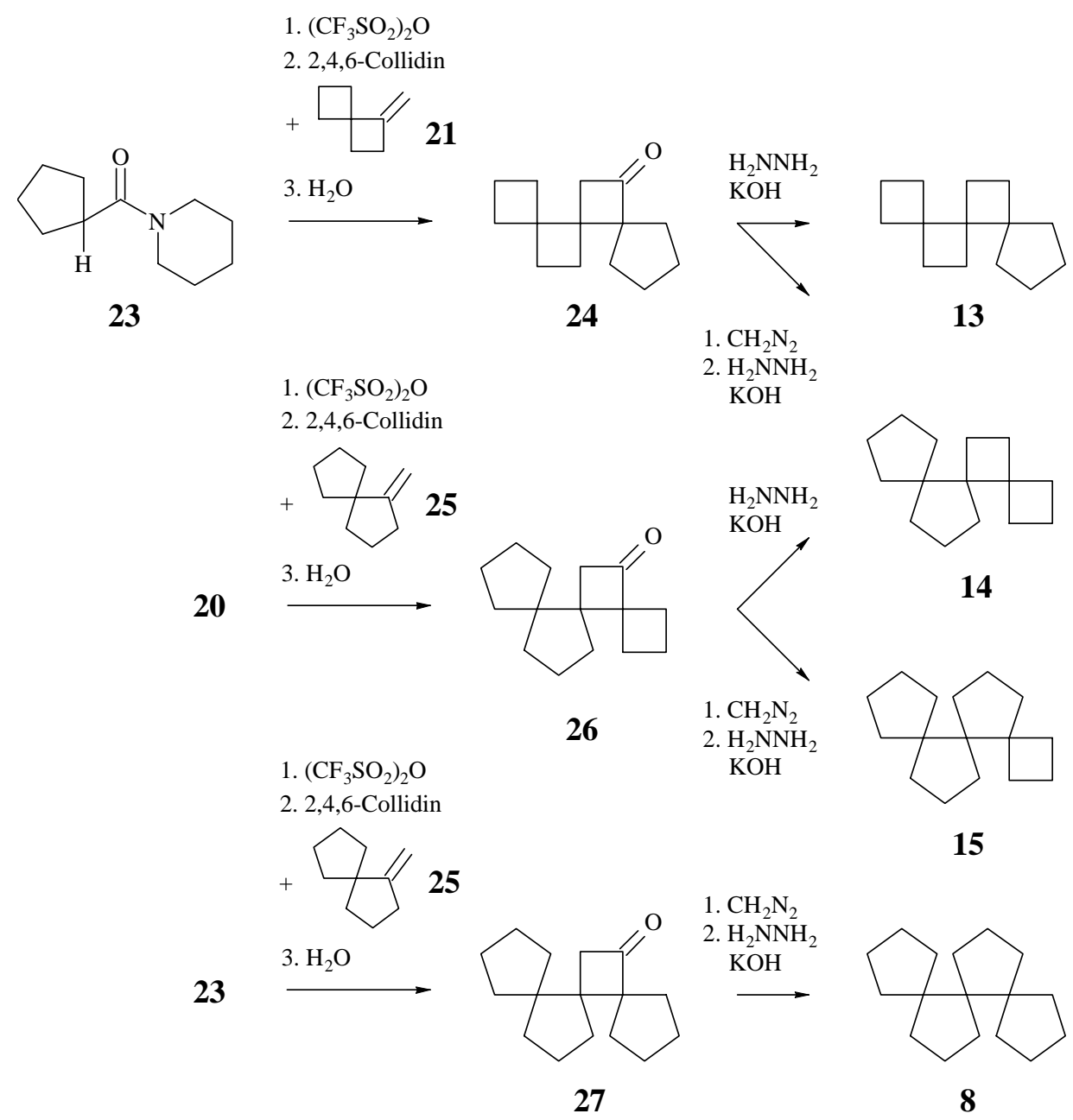


Wie später gezeigt werden wird, gelang von den angestrebten Cycloadditionen lediglich die zu 24. Damit war der Weg zu 13 und 14 offen, der zu 15 und 8 dagegen weiterhin verschlossen. Die Synthesen von rac-13 und rac-14 sowie von (5R)-13 und (5S)-14 gelangen wie angegeben (Schema 5) und basierten bei den optisch aktiven Vertretern auf dem nach erschöpfender Reduktion von rac-24 mit Bäckerhefe und Abtrennung der gebildeten Alkohole (5S,11S)-28 und (5R,11S)-29 rein zurückbleibenden Enantiomer (5R)-24 (Schema 6).

\section{Schema 6}

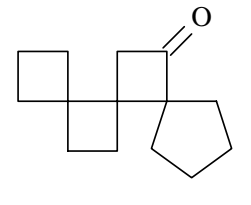

rac-24

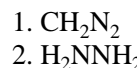

2. $\mathrm{H}_{2} \mathrm{NNH}_{2}$ $\mathrm{KOH}$

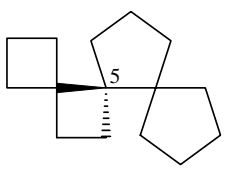

(5S)-14
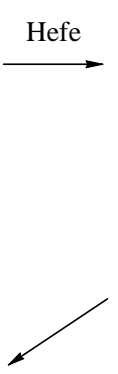

$\mathrm{H}_{2} \mathrm{NNH}_{2}$
$\mathrm{KOH}$

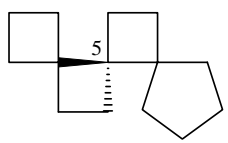

(5R)-13

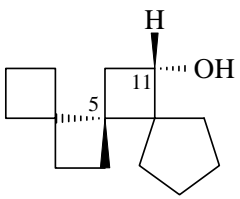

$(5 S, 11 S)-28(96 \%)$

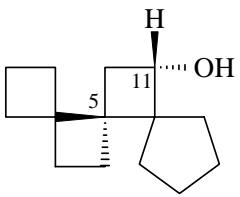

$(5 R, 11 S)-29(4 \%)$

Nach den Ergebnissen der Cycloadditionsversuche hielten wir es für geboten, in weiteren Versuchen zur Synthese von 15 und 8 die Zahl der zu anellierenden Ringe auf einen zu beschränken und von einem fertigen Dispiran auszugehen. Erste Wahl war das bekannte, aber schwer zugängliche Dispiroketon 3 30 $^{23}$ das über eine Reaktionsfolge aus Methylenierung, Addition von Dichlorketen ${ }^{24}$ und reduktiver Enthalogenierung ${ }^{24}$ (30-33-34) in 34 als direkten Vorläufer von 15, sowie, durch Ringerweiterung mit Diazomethan, ${ }^{25}$ in 35 und 36 als direkten Vorläufern von 8 überführbar schien. (Schema 7).

Für das benötigte Dispiran 30 wurde eine neue Synthese entwickelt. Hierzu wurde das bekannte Spiroketon $32^{26}$ auf einem um zwei Stufen verkürzten Weg zugänglich gemacht und nach Spiroalkylierung einer säurekatalysierten Keton-Keton-Umlagerung unterworfen (32-3130) (Schema 7). Diese Synthese kann mit der einzigen bisher bekannten Synthese für $\mathbf{3 0}^{23}$ durchaus konkurrieren, ist allerdings wegen der unbefriedigenden Ausbeute im Umlagerungsschritt noch nicht optimal. 


\section{Schema 7}

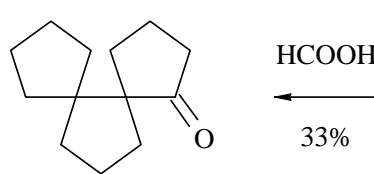

30

$\mathrm{Ph}_{3} \mathrm{P}=\mathrm{CH}_{2}$

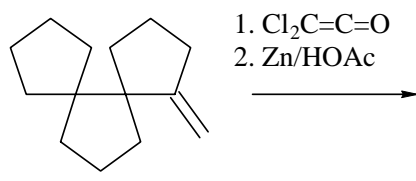

33

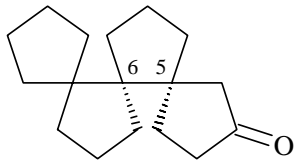

$\left(5 R^{*}, 6 R^{*}\right)-35$

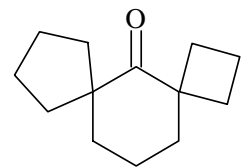

31

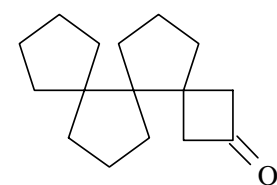

34

$\mathrm{CH}_{2} \mathrm{~N}_{2}$

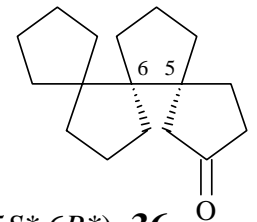

$\left(5 S^{*}, 6 R^{*}\right)-36$

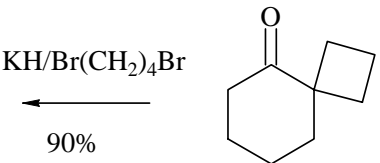

32

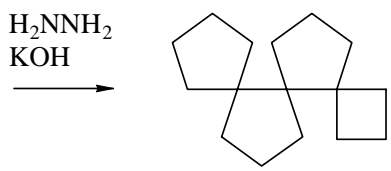

15

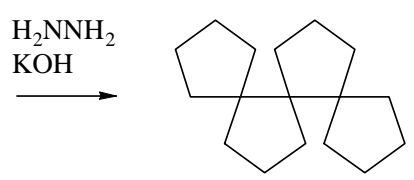

8

Leider reagierte das durch Methylenierung von $\mathbf{3 0}$ erhaltene $\mathbf{3 3}$ mit Dichlorketen zu einem offenkettigen Produkten, so dass Alternativen für eine Umwandlung von $\mathbf{3 0}$ in $\mathbf{1 5}$ und $\mathbf{8}$ geprüft werden mussten. Hierzu kamen alle gängigen Methoden zur Spiroanellierung von Cyclobutanonen in Betracht. ${ }^{27-34}$ Wie später gezeigt werden wird, scheiterten eine Reihe klassischer Verfahren, nicht jedoch eine von Baldwin ${ }^{35}$ entwickelte Methode, nach der Cyclohexanon (37) durch Addition von 1-Methoxy-1-vinyllithium, Cyclopropanierung und säurekatalysierte Umlagerung in Spiro[3.5]nonan-1-on (40) überführt werden kann (37-38-3940) (Schema 8).

\section{Schema 8}

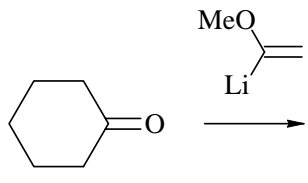

37

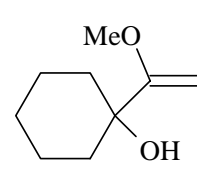

38

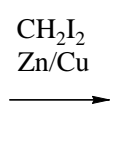

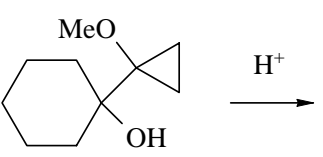

9

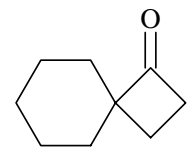

40

Mit 30 als Edukt resultierte ein stereochemisch einheitliches Dispiroketon 43 mit endo-ständiger Carbonylgruppe (30-41-42-43), das sich zwar deoxigenieren (43-15), nicht jedoch mit Diazomethan ringerweitern ließ (43-45+35). Immerhin gelang es, 8 auf dem Umweg einer Methylenierung, ${ }^{36}$ Epoxidierung, Oxaspirohexan-Cyclopentanon-Umlagerung ${ }^{37-39}$ und Deoxigenierung (43-44-46-35-8) zugänglich machen (Schema 9). Allerdings stieg dadurch 
die Zahl der Syntheseschritte auf sieben an. In Anbetracht einer nach wie vor nicht optimalen Synthese von 30 (Schema 7) sowie dem Befund, dass das zur Racemattrennung vorgesehenen (-)-DIP-Chlorid ${ }^{15}$ mit 30 nicht reagierte, zogen wir es vor, erneut nach kürzeren Alternativen für eine Synthese von $\mathbf{1 5}$ und $\mathbf{8}$ zu suchen.

\section{Schema 9}

\section{0}

1. $\mathrm{CeCl}_{3} / \mathrm{THF}$
2. $\mathrm{Li}-{ }_{\mathrm{OEt}}$
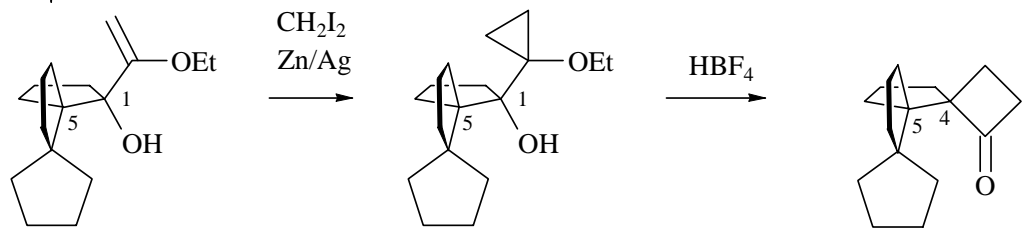

$\left(1 S^{*}, 5 S^{*}\right)-\mathbf{4 1}$

$\left(1 S^{*}, 5 S^{*}\right)-\mathbf{4 2}$

$\left(4 R^{*}, 5 S^{*}\right)-43$

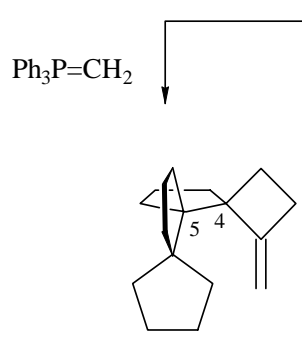

$\left(4 R^{*}, 5 S^{*}\right)-44$

$\left(5 R^{*}, 6 S^{*}\right)-\mathbf{4 5}$

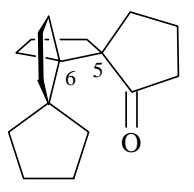

$\mathrm{H}_{2} \mathrm{NNH}_{2}$

$\mathrm{NaO}\left(\mathrm{CH}_{2}\right)_{2} \mathrm{O}\left(\mathrm{CH}_{2}\right)_{2} \mathrm{OH}$
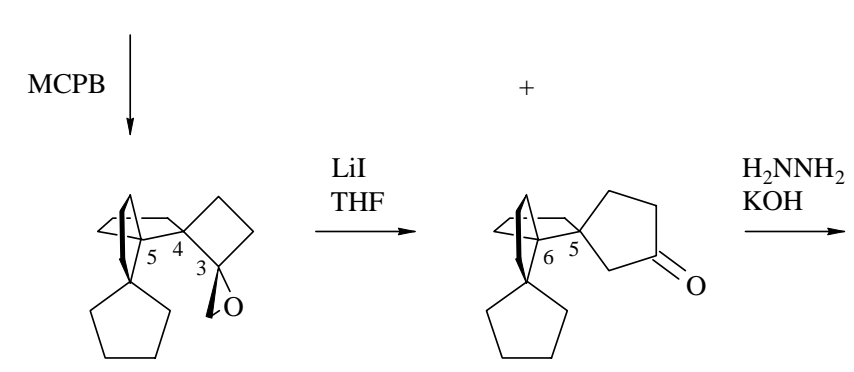

$\left(3 S^{*}, 4 R^{*}, 5 S^{*}\right)-46$

$\left(5 S^{*}, 6 S^{*}\right)-35$

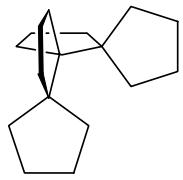

8

In diesem Stadium der Untersuchungen stießen wir auf eine Arbeit von Tu und Mitarbeitern ${ }^{40}$ über eine durch Halogen-Kationen induzierte stereoselektive Umlagerung von Allylalkoholen in $\beta$-Halogenketone. Eines der Beispiele beschrieb die Umlagerung von 1-(1Cyclopentenyl)-cyclobutanol (47) in 6-Brom-spiro[4.4]nonan-1-on (48) (Schema 10).

\section{Schema 10}

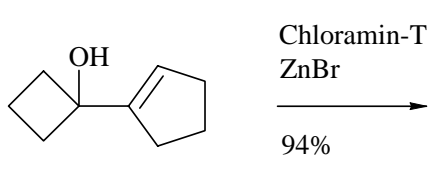

47

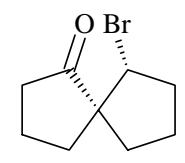

48 
Als weiteres potentielles Edukt einer Synthese von $\mathbf{8}$ und $\mathbf{1 5}$ ergab sich demnach das bis dahin unbekannte Dispiroketon 51 (Schema 11). Ausgehend von 50 gelang seine Synthese durch Cyclopropylidenierung, ${ }^{33,34}$ Epoxidierung und Oxaspiropentan-Cyclobutanon-Umlagerung $^{32}$ (50-49-51). Die nachfolgenden Additionen von Cyclopentenyllithium ${ }^{41}$ (51-52) und Cyclobutenyllithium $^{42}$ (51-53) verliefen stereoselektiv, und gleiches galt für die Umlagerungen zu 54 bzw. 56. Schwierigkeiten ergaben sich bei den reduktiven Enthalogenierungen: Lithiumaluminiumhydrid und Tri-n-butylzinnhydrid führten bei 54 zu ringgeöffneten bzw. umgelagerten Produkten, und nur mit Zink in Eisessig ${ }^{24}$ blieb das Gerüst intakt. Leider galt dies nicht mehr für 56. Damit war der zu 8 (51-52-54-55-8) geebnet, während für den Weg zu 15 erneut nach einer geeigneten Alternative gesucht werden mußte.

\section{Schema 11}

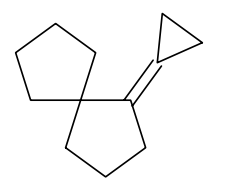

49

1. $\mathrm{MCPB}$

2. $\mathrm{BF}_{3} \cdot \mathrm{OEt}_{2}$

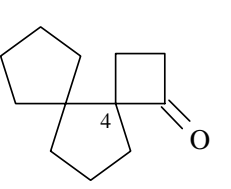

$\left(4 S^{*}\right)-51$

1. $\mathrm{CeCl}_{3} / \mathrm{THF}$

2. $\mathrm{Li} \gg$,

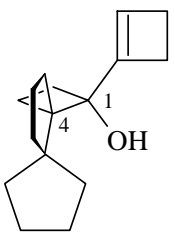

$\left(1 R^{*}, 4 S^{*}\right)-53$

Chloramin-T

$\mathrm{ZnBr}_{2}$

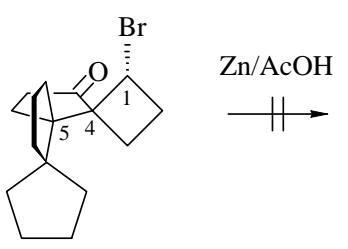

$\left(1 R^{*}, 4 S^{*}, 5 S^{*}\right)-56$

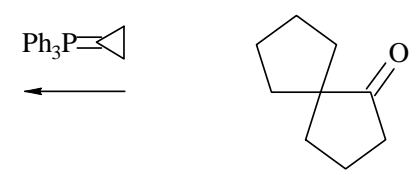

50

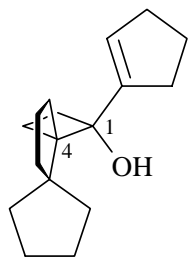

$\left(1 R^{*}, 4 S^{*}\right)-52$
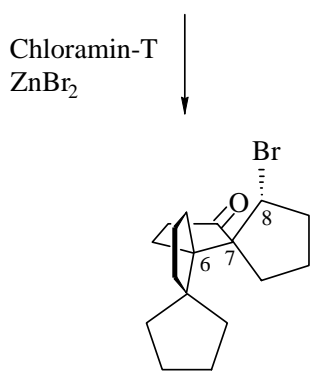

$\left(6 S^{*}, 7 S^{*}, 8 R^{*}\right)-54$
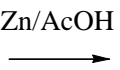

$\mathrm{H}_{2} \mathrm{NNH}_{2}$
$\mathrm{NaO}\left(\mathrm{CH}_{2}\right)_{2} \mathrm{O}\left(\mathrm{CH}_{2}\right)_{2} \mathrm{OH}$

8

1

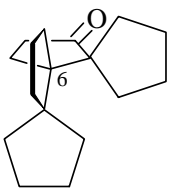

$\left(6 S^{*}\right)-55$

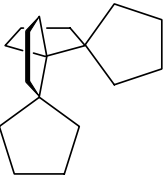


1-Lithio-1-methylselenocyclobutan addiert sich an cyclische Ketone unter Bildung von $\beta$-Hydroxyseleniden, die bei Oxidation mit 3-Chlorperbenzoesäure unter Abspaltung von Methylseleninsäure ein um einen spiroanellierten Cyclobutanring ringerweitertes Keton liefern. ${ }^{43}$ Das Reagenz wird durch reduktive Metallierung des Dimethylselenoketals von Cyclobutanon dargestellt, und dieses wiederum durch Zinkchlorid-katalysierte Umsetzung von Cyclobutanon mit dem leicht flüchtigen, übel riechenden und toxischen Methylselenol. $^{44,45}$ Das Reagenz sollte dementsprechend nur dann angewandt werden, wenn andere Mittel versagen.

Wie schon mit 1-Lithio-cyclobuten (Schema 11), reagierte 51 auch mit 1-Lithio-1-methylselenocyclobutan stereoselektiv und lieferte nach Oxidation des rohen $\beta$-Hydroxyselenids 58 mit 57 ein im gewünschten Sinne regioselektiv ringerweitertes Keton (Schema 12). Seine Deoxigenierung zu 15 erforderte drastische Bedingungen, ${ }^{46}$ war aber erfolgreich. Ausgehend von 51 stand damit eine vierstufige Synthese für 8 (Schema 11) und eine dreistufige Synthese für 15 (Schema 12) zur Verfügung.

\section{Schema 12}

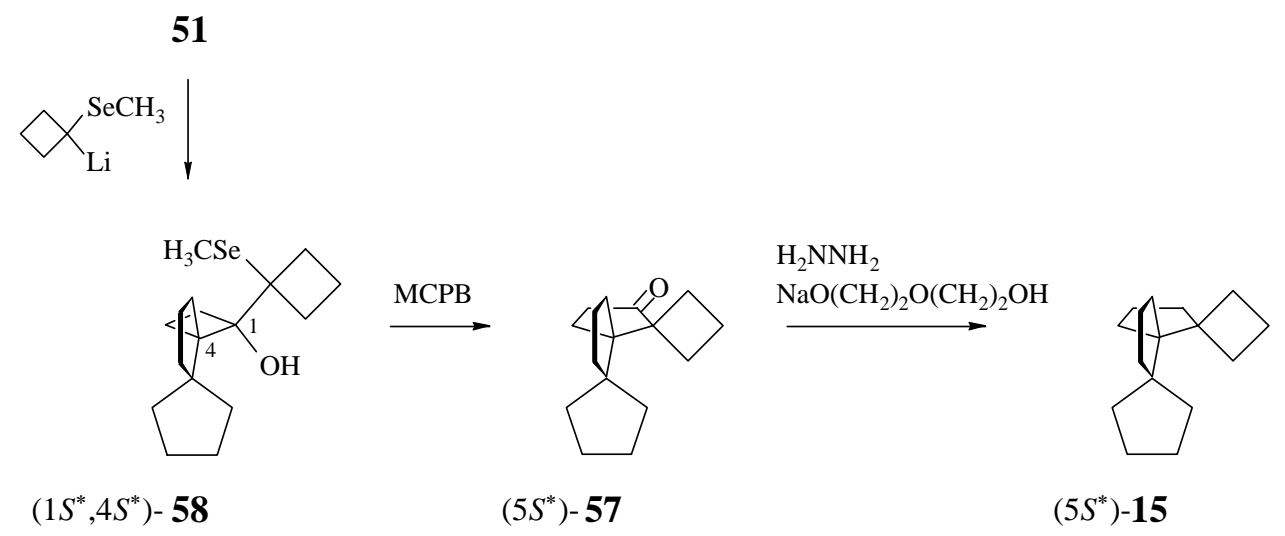

Das noch offene Problem einer Trennung der Enantiomeren von 51 wurde auf zwei Wegen gelöst (Schema 13): erstens, durch stereospezifische Addition von lithiiertem $(+)-(S)-$ $N, S$-Dimethyl-S-phenylsulfoximin, Trennung der Diastereomeren und thermische Fragmentierung $^{47,48}$ [rac-51-(SS,1R,4R)-59-(4R)-51 bzw. (SS,1S,4S)-61-(4S)-51], und zweitens durch enantioselektive Reduktion mit (-)-DIP-Chlorid, ${ }^{15}$ Trennung der Diastereomeren und Rückoxidation mit Pyridiniumchlorochromat ${ }^{16}$ [rac-51-(1S,4R)-60-(4R)-51 bzw. (1S,4S)-62(4S)-51]. Die über 59 und $\mathbf{6 1}$ dargestellten Ketone (4R)-51 und (4S)-51 waren enantiomerenrein (>99\% ee). Gleiches galt für über 60 dargestelltes (4S)-51 (>99\% ee), nicht jedoch für über 62 dargestelltes (4S)-51 (69\% ee). Für die asymmetrischen Synthesen wurde durchgängig enantiomerenreines (4R)-51 eingesetzt. 


\section{Schema 13}

$(-)-(\mathrm{SS})-\mathrm{C}_{6} \mathrm{H}_{5} \mathrm{~S}(\mathrm{O})\left(\mathrm{NCH}_{3}\right) \mathrm{CH}_{2} \mathrm{Li}$

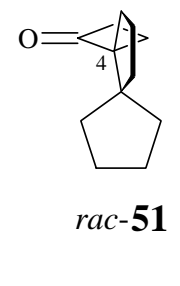

(-)-DIP-Cl

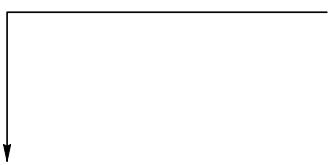

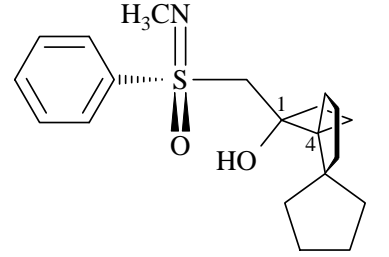

(SS,1R,4R)-59 (〉99\% eе)

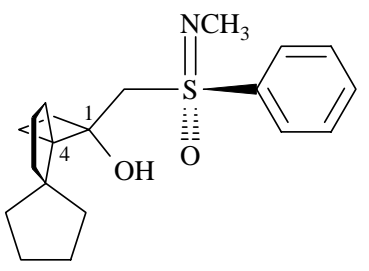

(SS,1S,4S)-61 (〉99\% ee)

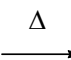

(4S)-51 (>99\% ee) (4S)-51 (69\% ee)
$(4 R)-51 \quad(>99 \%$ ee $)$

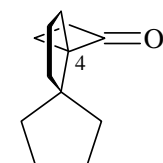

PCC

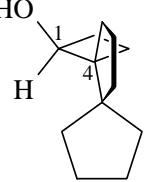

$(1 S, 4 R)-60 \quad(\rangle 99 \%$ ee $)$

CC

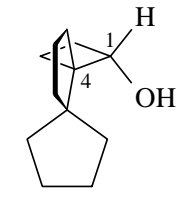

(1S,4S)-62 (69\% eе)

Zur Komplettierung der Reihe enantiomerenreiner Vertreter der Trispirane 13, 14, 15 und 8 wurden die für racemisches 15 (Schema 12) und racemisches 8 (Schema 11) ausgearbeiteten Synthesen mit (4R)-51 wiederholt. Dies lieferte mit (5R)-15 [("P")-15] und (P)-8 die beiden noch fehlenden Vertreter und erlaubte zusammen mit den im ersten Teil der Arbeit beschriebenen Trispiranen (5R)-13 [("P")-13] und (5-S)-14 [("P)-14)] sowie den bekannten Trispiranen $(M)-6^{1,2}$ und $(M)-7^{4}$ einen Gesamtvergleich von Vorzeichen und Betrag der spezifischen Drehung innerhalb der Reihe $(M)-6$, $(M)-7$, ("P")-13, ("P")-14, ("P")-15 und (P)8 (Schema 14). Insbesondere der Betrag und das Vorzeichen der spezifischen Drehung von $(P)$-8 sind unerwartet. 


\section{Schema 14}

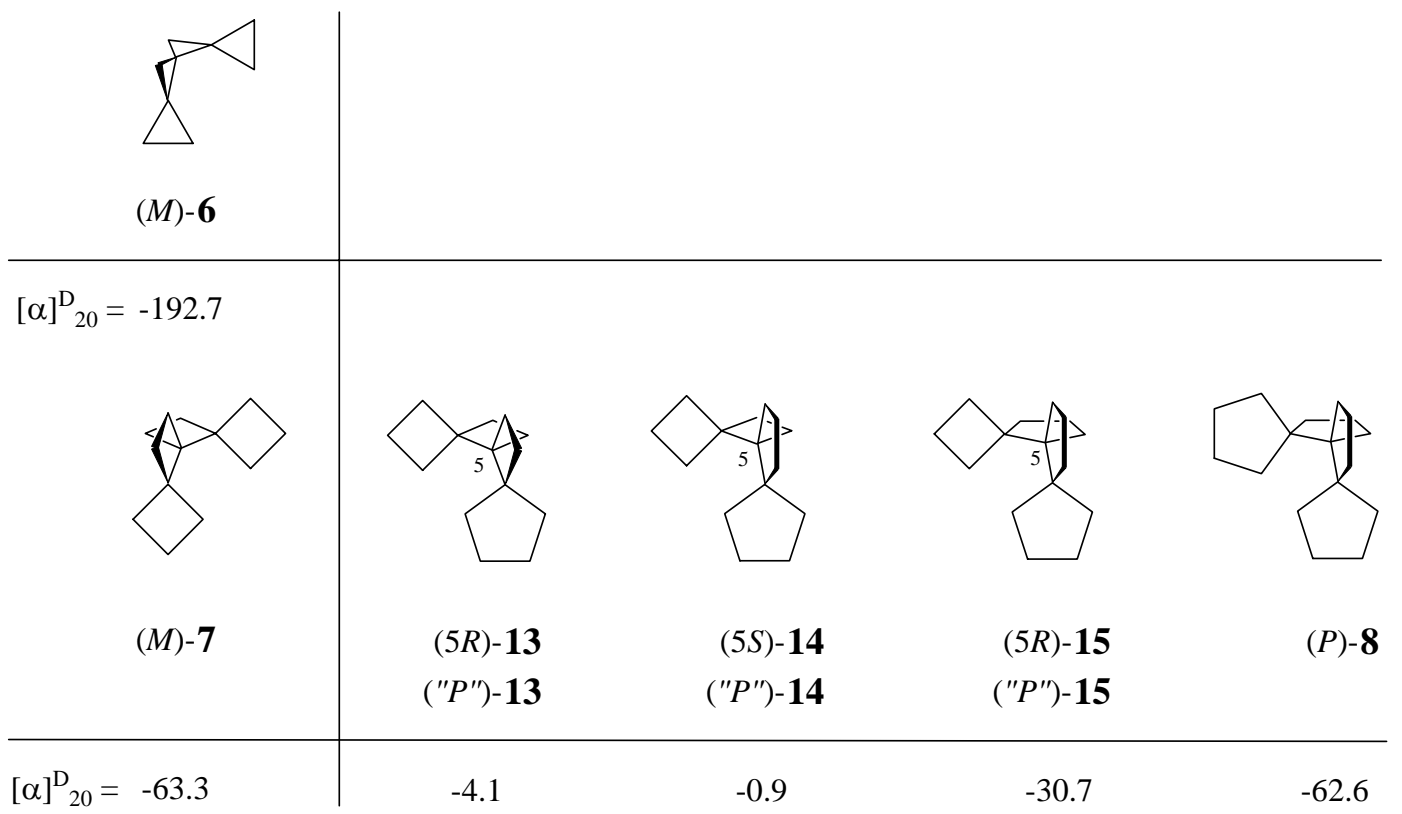

Die vorliegende Arbeit hatte zum Ziel, enantiomerenreine Vertreter der pseudohelicalen Trispirane 13, 14 und 15 sowie des helicalen Trispirans 8 darzustellen und ihre spezifische Drehung bei verschiedenen Wellenlängen zu bestimmen. Auf diese Weise sollte ermöglicht werden, über ab initio-Rechnungen der Frage nachzugehen, ob und in welchem Ausmaß konformationelle Einflüsse gegenüber dem Einfluß geometrischer Faktoren (Identitätsperiode, Ganghöhe und Länge der Helix) dominieren.

Unter Berücksichtigung der geschilderten Schwierigkeiten bei der Umsetzung der verschiedenen Synthesen gliedert sich die Arbeit wie folgt: der erste Teil beschreibt die Cycloadditionsversuche mit Keteniminiumsalzen und die racemischen Synthesen von $\mathbf{1 3}$ und 14. Der zweite Teil beschreibt die Racemattrennung auf der Stufe von $\mathbf{2 4}$ sowie die enantioselektiven Synthesen von (5R)-13 und (5S)-14. Der dritte Teil schildert die verschiedenen Alternativen für racemische Synthesen von $\mathbf{1 5}$ und $\mathbf{8}$, und der vierte Teil die Racemattrennung auf der Stufe von 51 sowie die enantioselektiven Synthesen von (5R)-15 und $(P)$-8. Der fünfte Teil beschreibt die Kristallstrukturanalysen, und der sechste und letzte Teil befasst sich mit den chiroptischen Eigenschaften der dargestellten pseudohelicalen bzw. helicalen Trispirane (5R)-13, (5S)-14, (5R)-15 und (P)-8 und diskutiert die Ergebnisse. 


\section{ALLGEMEINER TEIL}

1. Versuche zur Cycloaddition von Keteniminiumsalzen an 1-Methylenspiro[3.3]heptan (21) und 1-Methylenspiro[4.4]nonan (25): Synthese von (5R*)-Trispiro[3.0.0.4.2.2]tetradecan [(5R*)-13] und $\left(5 R^{*}\right)$-Trispiro[3.0.0.4.3.2]pentadecan $\left[\left(5 R^{*}\right)-14\right]$

Wie einleitend näher ausgeführt, sollten in der vorliegenden Arbeit die Cyclobutane des helicalen Trispirans $7^{4,5}$ schrittweise durch Cyclopentane ersetzt und die chiroptischen Eigenschaften der resultierenden Trispirane 13, 14, 15 und 8 untersucht werden (Schema 15). Ziel war es, die für eine spätere ab-initio-Untersuchung der Abhängigkeit des Drehvermögens von statischen (Identitätsperiode, Ganghöhe und Länge der Helix) und dynamischen Parametern (konformationelle Gleichgewichte) notwendigen experimentellen Daten zur Verfügung zu stellen.

\section{Schema 15}

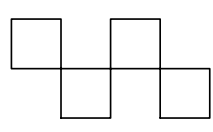

7

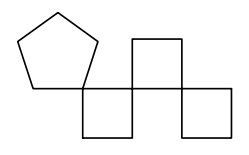

13

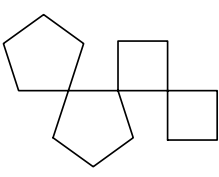

14

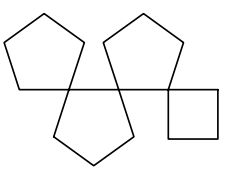

15

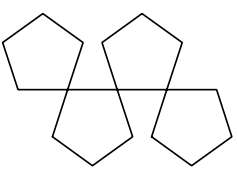

8

Bezüglich der Synthesen von 13, 14, 15 und 8 war geplant, die Gerüste analog der Synthese von 7 (20-63-22-7) ${ }^{5}$ durch Cycloaddition von Keteniminiumsalzen an Methylenspiroalkane darzustellen (Schema 16). Hierzu sollte das bisher unbekannte Keteniminiumsalz 64 aus dem ebenfalls unbekannten Amid 23 erzeugt und sowohl an 1-Methylenspiro[3.3]heptan (21) wie an 1-Methylenspiro[4.4]nonan (25) addiert werden [23-64-24(27)]. Darüber hinaus sollte auch das bereits früher erzeugte Keteniminiumsalz 63 mit 1-Methylenspiro[4.4]nonan (25) umgesetzt werden (20-63-26). Die Umwandlung der als Produkte erhofften Trispiroketone 24, 26 und 27 in 13(14), 14(15) bzw. 8 schien unproblematisch, und eine Racemattrennung auf der Stufe der Ketone möglich.

Von den benötigten Reagenzien waren das Amid $\mathbf{2 0}^{5}$ und die Methylenspiroalkane $21^{5}$ und $\mathbf{2 5}^{19-22}$ bekannt. Die Synthesen von 25 schienen allerdings nicht optimal und sollten verkürzt werden. Das bisher unbekannte Amid 23 schien nach Standardverfahren leicht zugänglich. 


\section{Schema 16}
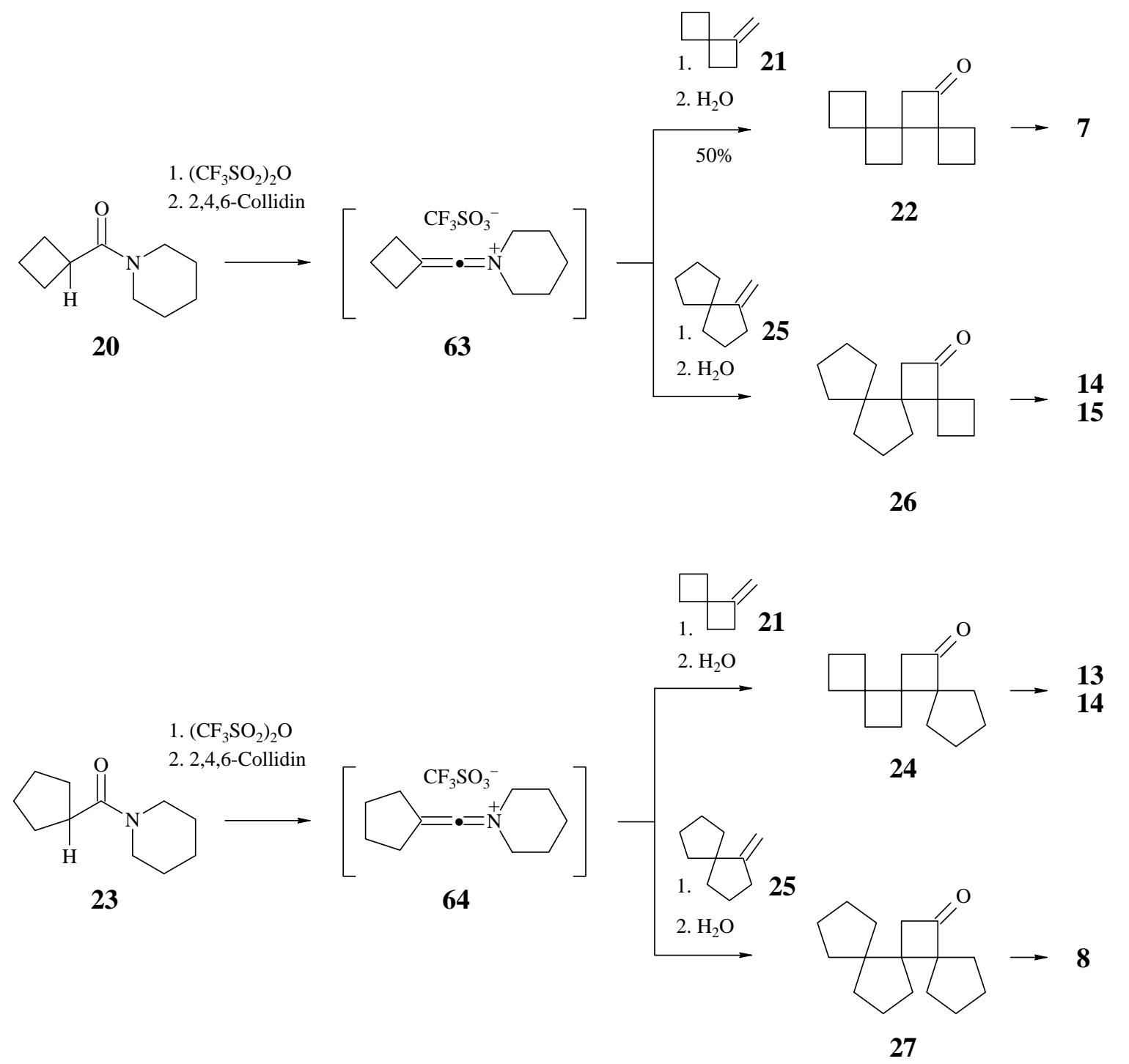

\subsection{Synthese der Vorprodukte}

1-Methylenspiro[4.4]nonan (25) wurde bisher erst zweimal synthetisiert: (a) durch Umsetzung eines aus 1,2-Dimethylen-cyclopentan (67) mit aktiviertem Magnesium generierten Komplexes mit 1,3-Dibrompropan (67-25), ${ }^{21,22}$ und (b) durch Olefinierung von Spiro[4.4]nonan-1-on (71) mit aus Methyltriphenylphosphoniumbromid und Natriumhydrid in DMSO generiertem Methylentriphenylphosphoran (71-25) ${ }^{19,20}$ (Schema 17). Der Weg zu 67 beginnt mit Cyclopentanon (66) und besteht aus einer Aminomethylierung, einer Methylenierung und einem Hoffmann-Abbau (66-67), ${ }^{49}$ der Weg zu 71 beginnt mit dem Kaliumsalz von 2-Oxo-cyclopentancarbonsäureethylester (72) und besteht aus einer Alkylierung, einer Verseifung und Decarboxylierung, sowie einer Cyclisierung (72-71). ${ }^{50}$ 


\section{Schema 17}

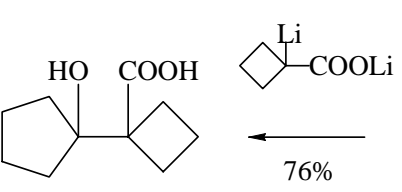

65

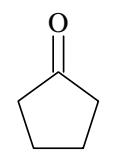

66

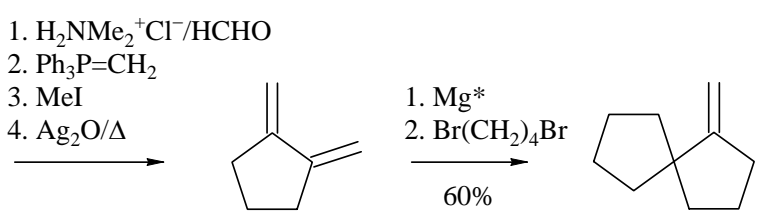

67
25

$$
\begin{aligned}
& \mathrm{PhSO}_{2} \mathrm{Cl} \\
& \mathrm{C}_{5} \mathrm{H}_{5} \mathrm{~N}
\end{aligned} \mid \quad \diamond=\mathrm{PPh}_{3} \downarrow 71 \% \quad \mathrm{PPh}_{3}=\mathrm{CH}_{2} \uparrow 95 \%
$$

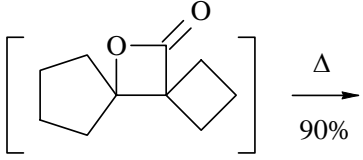

68

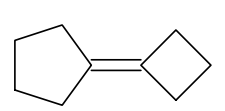

69

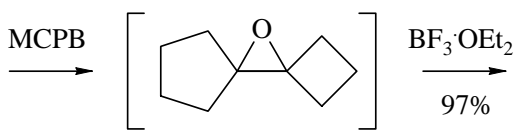

70

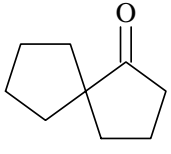

71

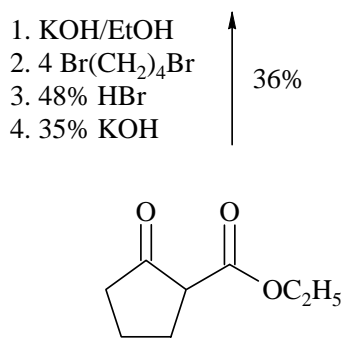

72

Wir haben Spiro[4.4]nonan-1-on (71) in operativ einfacher Weise durch Cyclobutylidenierung von Cyclopentanon (66) und anschließende Epoxidierung und in situ-Umlagerung des gebildeten Oxaspirohexans 70 zugänglich gemacht (66-69-70-71), und die nachfolgende Methylenierung zu 25 durch Verwendung von mit Kalium-tert-butylat generiertem Methylentriphenylphosphoran ${ }^{36}$ deutlich verbessert (95 vs. $74 \%{ }^{20}$ ) (Schema 17). Durch die direkte Cyclobutylidenierung von 66 zu 69 wurde ein in der Literatur ${ }^{51}$ beschriebener Weg über die $\beta$-Hydroxycarbonsäure $\mathbf{6 5}$ und das Lacton 68 verzichtbar und die Synthese von 71 deutlich verkürzt.

Zur experimentellen Realisierung wurde aus 4-Brombutyl-triphenylphosphonium-bromid mit zwei Äquivalenten Kalium-tert-butylat in Benzol generiertes Cyclobutyliden-triphenylphosphoran $^{52}$ mit Cyclopentanon (66) umgesetzt und das gebildete Cyclobutyliden-cyclopentan (69) nach Epoxidierung mit 3-Chlorperbenzoesäure mit katalytischen Mengen Bortrifluorid-Etherat umgelagert. Es resultierte in nahezu quantitativer Ausbeute das gewünschte Spiro[4.4]nonan-1-on (71), das mit aus Methyltriphenylphosphoniumbromid mit Kalium-tert-butylat in Ether generiertem Methylentriphenylphosphoran ${ }^{36}$ in wiederum nahezu quantitativer Ausbeute 1-Methylenspiro[4.4]nonan (25) lieferte. 


\section{Schema 18}

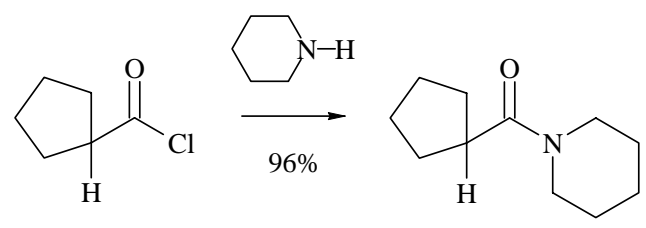

73

23

Das noch fehlende Amid 23 war durch Umsetzung von Cyclopentancarbonsäurechlorid (73) mit Piperidin leicht zugänglich (Schema 18). Interessant ist das ${ }^{13} \mathrm{C}-\mathrm{NMR}-S p e k t r u m$ (Abb. 90). Es zeigt für die Kohlenstoffatome des Piperidylrestes den vollen Satz von fünf Resonanzen [ $\delta=24.51(\mathrm{t}), 25.45(\mathrm{t}), 26.48(\mathrm{t}), 42.65(\mathrm{t}), 46.31(\mathrm{t})]$, für die Kohlenstoffatome des Cyclopentylrestes dagegen lediglich drei Resonanzen [ $\delta=25.81(\mathrm{t}), 29.92(\mathrm{t}), 40.87$ (d)]. Demnach ist die Rotation des Piperidylrestes, nicht jedoch die des Cyclopentylrestes bei Raumtemperatur behindert. Dies deckt sich mit Beobachtungen an 20. ${ }^{5}$

\subsection{Versuche zur Cycloaddition von Keteniminiumsalzen an Methylenspiroalkane}

Nachdem die benötigten Edukte synthetisiert waren, prüften wir, ob der Plan einer Synthese der Trispiroketone 24, 26 und 27 über eine Cycloaddition von Keteniminiumsalzen $(63,64)$ an Methylenspiroalkane $(\mathbf{2 1}, \mathbf{2 3})$ (vgl. Schema 16) realisierbar sein würde. Bezüglich der experimentellen Bedingungen orientierten wir uns an der erfolgreichen Cycloaddition von 63 an $21 .^{5}$

Als erstes prüften wir die Möglichkeit einer Cycloaddition des Keteniminiumsalzes 64 an 1-Methylenspiro[3.3]heptan (21). Hierzu wurde eine Lösung des Amids 23 in Dichlormethan in Gegenwart von 2.0 Äquivalenten 21 mit 1.2 Äquivalenten Trifluormethansulfonsäureanhydrid und 1.3 Äquivalenten 2,4,6-Collidin 20 h unter Rückfluß erhitzt. Hydrolyse und Chromatographie lieferten das erwünschte Trispiroketon 24 in einer Ausbeute von 21\% (Schema 19).

\section{Schema 19}

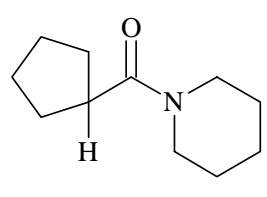

23
1. $\left(\mathrm{CF}_{3} \mathrm{SO}_{2}\right)_{2} \mathrm{O}$

2. 2,4,6-Collidin

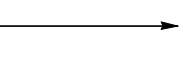

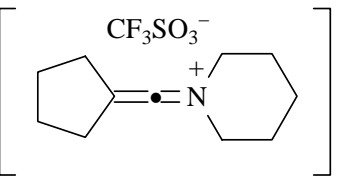

64

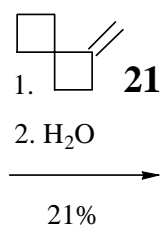

21

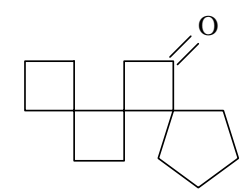

24 
Im IR-Spektrum (Abb. 13) findet sich eine für Cyclobutanone charakteristische Carbonylfrequenz bei $1770 \mathrm{~cm}^{-1}$, und im ${ }^{1} \mathrm{H}-\mathrm{NMR}$-Spektrum (Abb. 50) für die Protonen in Nachbarschaft zur Carbonylgruppe das erwartete AB-System bei tiefstem Feld ( $\delta=2.62$, d, J $=16.5 \mathrm{~Hz}, 1 \mathrm{H}$ und 3.12, d, $\mathrm{J}=16.5 \mathrm{~Hz}, 1 \mathrm{H}$ ). Das ${ }^{13} \mathrm{C}-\mathrm{NMR}-$ Spektrum (Abb. 91) enthält die für 24 zu fordernden vierzehn Resonanzen für zehn sekundäre und vier quartäre Kohlenstoffatome, darunter die Resonanz für das Carbonylkohlenstoffatom bei $\delta=214.10$.

Ersetzte man 1-Methylenspiro[3.3]heptan (21) unter sonst identischen Bedingungen durch 1-Methylenspiro[4.4]nonan (25), so führten sämtliche Versuche zur Cycloaddition an 63 und 64 zu weitgehender Polymerisation. Die als Produkte erhofften Trispiroketone 26 bzw. 27 konnten nicht nachgewiesen werden. Stattdessen isolierten wir aus dem niedermolekularen Anteil der Umsetzung von 64 mit 25 in sehr geringer Menge zwei nicht ganz saubere offenkettige Ketone, bei denen es sich nach Ausweis ihrer ${ }^{1} \mathrm{H}$ - (Abb. 77, 76) und ${ }^{13} \mathrm{C}-\mathrm{NMR}$ Spektren (Abb. 119, 118) in Verbindung mit ${ }^{3} \mathrm{~J}_{\mathrm{HH}}$-Korrelationsspektren um 76 (3\%) und 75 (1\%) handelte (Schema 20).

\section{Schema 20}

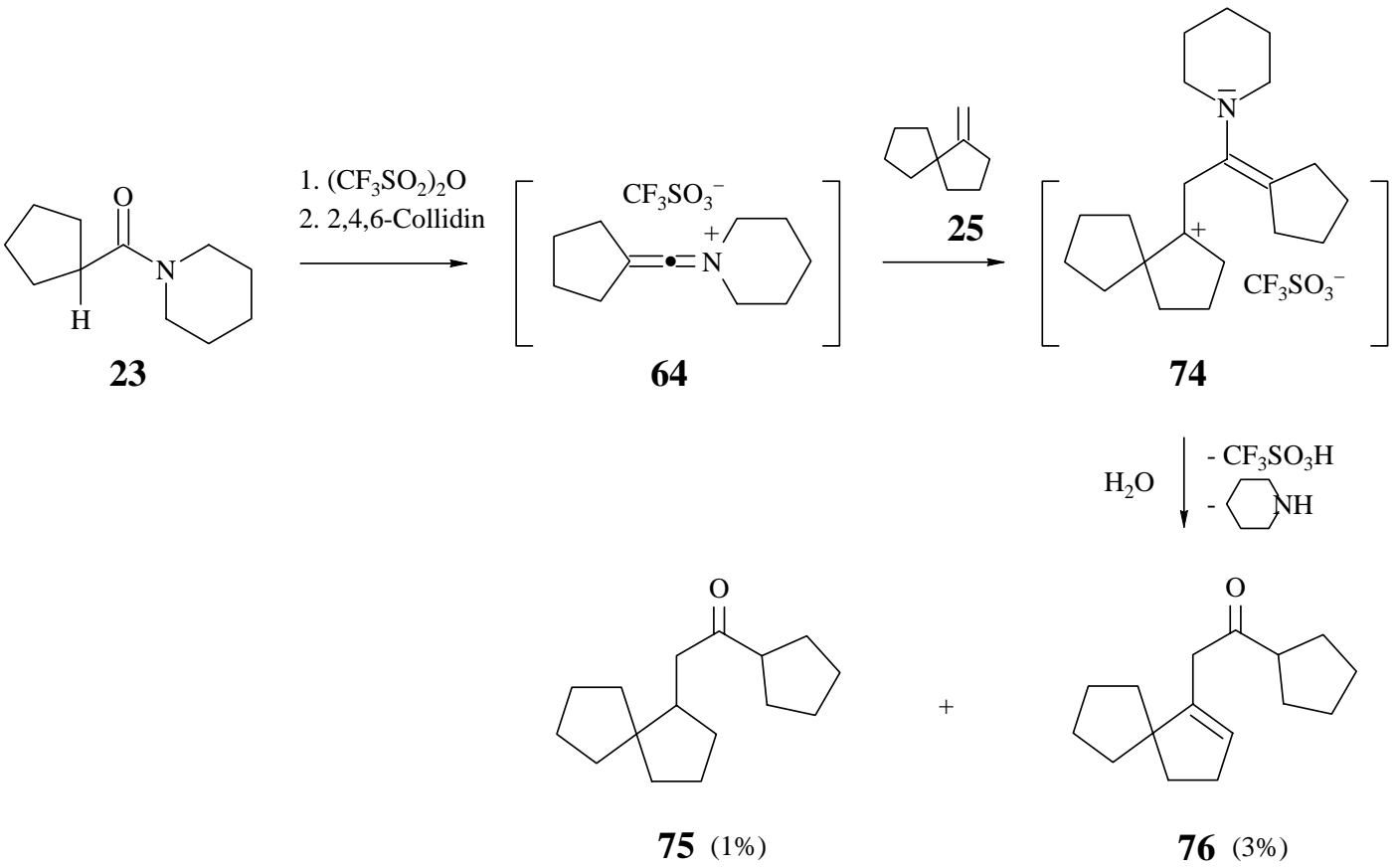

Bezüglich der Produktbildung nehmen wir an, dass sich das Keteniminiumsalz 64 an 25 unter Bildung von $\mathbf{7 4}$ addiert, dieses aber nicht cyclisiert, sondern durch Abspaltung eines Protons und Hydrolyse in $\mathbf{7 6}$ übergeht. Ob die als Hauptreaktion beobachtete Polymerisation bereits auf der Stufe von $\mathbf{7 4}$ und/oder davon abgeleiteter Diene erfolgt, oder aber erst im Zuge der Verseifung eintritt, wissen wir nicht. Der Grund für das Auftreten von 75 ist ebenfalls unklar. 
Zusammenfassend ist festzustellen, dass die Keteniminiumsalze 63 und 64 mit 1-Methylenspiro[3.3]heptan (21), nicht jedoch mit 1-Methylenspiro[4.4]nonan (25) Cycloadditionen eingehen. Das auf diese Weise zugängliche Trispiroketon 24 öffnete den Weg zu den Trispiranen 13 und 14, während für die Trispirane 15 und 8 andere Vorläufer gefunden werden mussten.

\subsection{Synthese von (5R*)-Trispiro[3.0.0.4.2.2]tetradecan [(5R*)-13] und (5R*)- Trispiro[3.0.0.4.3.2]pentadecan [(5R*)-14]}

Zur Darstellung des Trispirans 13 musste 24 lediglich reduziert werden. Dies erfolgte nach Wolff-Kishner in der Variante von Huang-Minlon. ${ }^{18}$ Erhitzte man 24 mit Hydrazinhydrat und gepulvertem $\mathrm{KOH}$ in Diethylenglykol auf $160^{\circ} \mathrm{C}$, so war die Reduktion nach $1.5 \mathrm{~h}$ komplett. Aufarbeitung und Filtration über Kieselgel in Pentan lieferten $\mathbf{1 3}$ in einer isolierten Ausbeute von 82\% (Schema 21). Der Erfolg der Reduktion war im IR- (Abb. 9), ${ }^{1} \mathrm{H}-\mathrm{NMR}$ (Abb. 46) und ${ }^{13}$ C-NMR-Spektrum (Abb. 87) an dem Verschwinden der durch die Carbonylgruppe verursachten Signale leicht ablesbar.

\section{Schema 21}

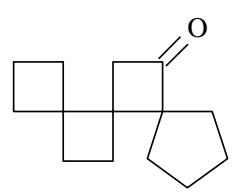

24

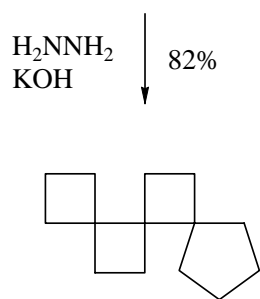

13

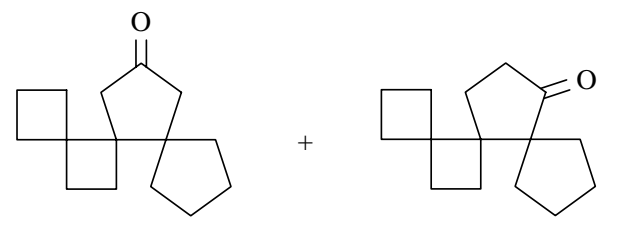

78
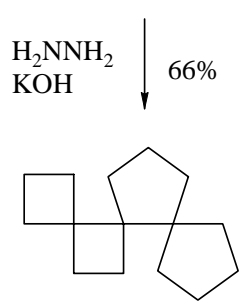

14

Zur Homologisierung von Ketonen sind zahlreiche Methoden bekannt. ${ }^{25,53-56}$ Die älteste verwendet Diazomethan, ${ }^{25,53}$ das über eine Methylen-Insertion nicht regiospezifisch ringerweiterte Ketone ergibt. Bei $\mathbf{2 4}$ war dies ohne Belang, da die zu erwartenden Ketone $\mathbf{7 7}$ und $\mathbf{7 8}$ im nächsten Schritt ohnehin deoxygeniert werden sollten. Setzte man $\mathbf{2 4}$ mit in situ aus $p$-Toluolsulfonylmethylnitrosamid (Diazald ${ }^{\circledR}$ ) mit Kaliumhydroxid in wässrig-methanolischer Lösung erzeugtem Diazomethan um, so erhielt man bei rechtzeitigem Abbruch in 71\% Ausbeute ein 55:45-Gemisch der Ketone 77 und 78 (Reinheit 93\% GC). Die beiden 
Ketone waren gaschromatographisch, nicht aber säulenchromatographisch trennbar und anhand der ${ }^{1} \mathrm{H}-\mathrm{NMR}$-Resonanzen für die der Carbonylgruppe benachbarten Protonen leicht unterscheidbar: 77 (Abb. 78) zeigte die erwarteten zwei AB-Systeme ( $\delta=1.95, \mathrm{~d}, \mathrm{~J}=18 \mathrm{~Hz}$, $1 \mathrm{H}$ und 2.16, d, $\mathrm{J}=18 \mathrm{~Hz}, 1 \mathrm{H}$ sowie $\delta=2.18$, d, $\mathrm{J}=18 \mathrm{~Hz}, 1 \mathrm{H}$ und 2.37, d, $\mathrm{J}=18 \mathrm{~Hz}, 1 \mathrm{H}$ ), 78 (Abb. 79) dagegen wegen zufällig identischer chemischer Verschiebungen und Kopplungskonstanten ein pseudo-Triplett $(\delta=2.21$, dd, $\mathrm{J}=7.5,7.5 \mathrm{~Hz}, 2 \mathrm{H})$.

Im Vergleich zu $\mathbf{2 4}$ war die Reduktion von $\mathbf{7 7}$ und $\mathbf{7 8}$ erheblich schwieriger. Erhitzte man das Gemisch der beiden Ketone mit Hydrazinhydrat und gepulvertem $\mathrm{KOH}$ in Diethylenglykol $2 \mathrm{~h}$ auf $120^{\circ} \mathrm{C}$, und dann auf $195-200^{\circ} \mathrm{C}$, so erfolgte die Reduktion von 77 relativ schnell. Die des sterisch stärker gehinderten 78 war dagegen erst nach 5 h komplett. Aufarbeitung und Chromatographie an Kieselgel in Pentan lieferte $\mathbf{1 4}$ in einer Ausbeute von 66\% (Reinheit 87\% GC). Analysenreine Proben erhielt man durch präparative Gaschromatographie. Die ${ }^{1} \mathrm{H}$ - (Abb. 47) und ${ }^{13} \mathrm{C}-\mathrm{NMR}-$ Spektren (Abb. 88) entsprachen der Erwartung.

\section{Racemattrennung von (5R*)-Trispiro[3.0.0.4.2.2] tetradecan-11-on [(5R*)-24] durch enantioselektive Reduktion: Synthese von (5R)-Trispiro[3.0.0.4.2.2]- tetradecan [(5R)-13] und (5S)-Trispiro[3.0.0.4.3.2]pentadecan [(5S)-14]}

\subsection{Racemattrennung von (5R*)-Trispiro[3.0.0.4.2.2]tetradecan-11-on [(5R*)-24]}

Nach Komplettierung der racemischen Synthesen sollten 13 und 14 enantiomerenrein dargestellt werden. Dazu musste auf der Stufe des Trispiroketons 24 eine Racemattrennung vorgenommen werden. Aufgrund früherer Erfahrungen sollte hierzu eine asymmetrische Reduktion entweder chemisch mit (-)-Diisopinocampheylchloroboran [(-)-DIP-Cl] ${ }^{15}$ oder enzymatisch mit Bäckerhefe ${ }^{14}$ versucht werden. Anschließende Rückoxidation mit Pyridiniumchlorochromat ${ }^{16}$ sollte dann enantiomerenreines 24 liefern.

Um Kenntnisse über die zu erwartenden Alkohole zu erhalten, wurde $\mathbf{2 4}$ zunächst mit Lithiumaluminiumhydrid reduziert. Dies lieferte ein 65:35-Gemisch zweier diastereomerer Alkohole (Schema 22), die allerdings weder säulenchromatographisch noch präparativ gaschromatographisch trennbar waren. Damit schied (-)-DIP-Cl als enantioselektives, aber nicht diastereoselektives Reagenz zur asymmetrischen Reduktion aus. Sein Einsatz hätte eine Trennbarkeit der diastereomeren Alkohole vorausgesetzt.

Als nächstes stellte sich die Frage nach der Dia- und Enantioselektivität einer Reduktion mit Bäckerhefe. Zur Etablierung der notwendigen Analytik untersuchten wir die mit Lithiumaluminiumhydrid erhaltenen racemischen Alkohole kapillargaschromatographisch an Octakis(2,6-di-O-pentyl-3-O-butyryl)- $\gamma$-cyclodextrin als chiraler Phase. Daran wurden die 
Enantiomeren des Majoritätsalkohols getrennt, während die Enantiomeren des Minoritätsalkohols ungetrennt blieben. Wir mussten deshalb hoffen, dass 24 durch Hefe (a) enantioselektiv, und (b) mit derselben Diastereoselektivität wie mit Lithiumaluminiumhydrid reduziert werden würde, da sonst die Enantiomerenreinheit nicht hätte bestimmt werden können.

\section{Schema 22}

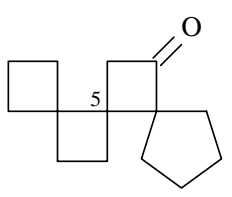

$\left(5 R^{*}\right)-24$

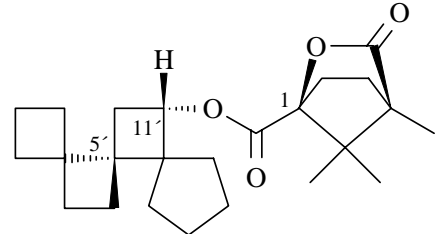

$\left(1 S, 5^{\prime} S, 11^{\prime} S\right)-79$

\begin{tabular}{l|ll|l} 
(a) $\mathrm{LiAlH}_{4}$ & $97 \%$ & $\begin{array}{l}\text { (1S)-Camphansäure- } \\
\text { (b) Hefe }\end{array}$ & $42 \%$
\end{tabular}$\quad \begin{aligned} & \text { chlorid/ } \mathrm{C}_{5} \mathrm{H}_{5} \mathrm{~N} \\
& 69 \%\end{aligned}$

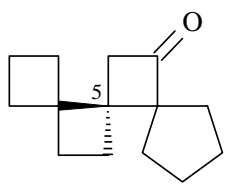

(a)

(b) $\quad(5 R)-24$

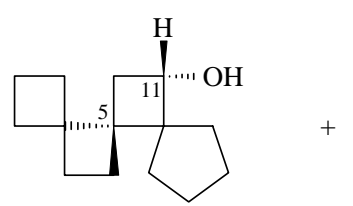

rac- $\mathbf{2 8}$

$(5 S, 11 S)-28$

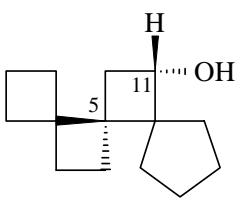

rac-29 $(5 R, 11 S)-29$

Zur Reduktion selbst wurde eine Lösung von 24 in Ethanol in eine Suspension von Hefe und Zucker in Wasser gegeben und das Gemisch bei $35^{\circ} \mathrm{C}$ gerührt. Nach 8 h setzte man weitere Hefe, Zucker und Wasser zu und arbeitete nach 22 h durch Verdünnen mit Wasser und kontinuierliche Extraktion mit Ether auf. Dies lieferte einen Rohextrakt, der laut gaschromatographischer Analyse 58\% nicht umgesetztes Keton und 42\% eines 96:4-Gemisches zweier diastereomerer Alkohole enthielt. Der Majoritätsalkohol war mit dem Majoritätsalkohol der Lithiumaluminiumhydrid-Reduktion identisch und enantiomerenrein. Zur Bestimmung der absoluten Konfiguration wurde eine Probe mit (-)-(1S)-Camphansäurechlorid verestert, der Minoritätsester durch fraktionierte Kristallisation abgetrennt, und der Majoritätsester kristallographisch untersucht (vgl. 5, Abb. 1). Als Resultat ergab sich dessen absolute Konfiguration als (1S,5'S,11'S)-79 und damit die des zugehörigen Majoritätsalkohols als (5S,11S)-28 (Schema 22). Eine S-selektive Reduktion wurde bereits an dem mit 24 vergleichbaren 22 beobachtet und stimmt mit der Prelog'schen Regel ${ }^{57}$ überein. Die Zuordnung der absoluten Konfiguration des Minoritätsalkohols als $(5 R, 11 S)$-29 basiert auf der Annahme einer ebenfalls $S$-selektiven Reduktion und ist vorläufig.

An dieser Stelle war zu entscheiden, ob das 96:4-Gemisch der diastereomeren Alkohole $(5 S, 11 S)$-28 und $(5 R, 11 S)-29$ (Fp. $47-52^{\circ} \mathrm{C},[\alpha]_{\mathrm{D}}{ }^{20}=+33.6, \mathrm{c}=1.10$, Aceton) oxidiert 
und damit eine verminderte optische Aktivität des resultierenden Ketons (5S)-24 akzeptiert werden sollte, oder ob das Minoritätsenantiomer der durch Chromatographie zurückgewonnenen 83:17-Mischung von (5R)-24 und (5S)-24 durch weitere Reduktion eliminiert werden sollte. Wir entschieden uns für letzteres. Eine viertägige zweite Reduktion lieferte bei 28\% Umsatz ein 58:42-Gemisch aus (5S,11S)-28 und (5R,11S)-29, und damit ein 99:1-Gemisch aus (5R)-24 und (5S)-24 als zurückgewonnenem Keton. Eine zweitägige dritte Reduktion führte bei 12\% Umsatz zu einem 5:95-Gemisch aus (5S,11S)-28 und (5R,11S)-29 (Fp. 78$82^{\circ} \mathrm{C},[\alpha]_{\mathrm{D}}{ }^{20}=+62.5, \mathrm{c}=1.13$, Aceton), und damit zu enantiomerenreinem (5R)-24 (>99\% ee, $[\alpha]_{\mathrm{D}}{ }^{20}=-0.5, \mathrm{c}=1.13$, Aceton). Seine ${ }^{1} \mathrm{H}$ - und ${ }^{13} \mathrm{C}$-NMR-spektroskopischen Daten stimmten mit denen racemischen Materials überein.

Die spektroskopischen Daten der präparativ nicht trennbaren diastereomeren Alkohole 28 und 29 wurden den im Früh- bzw. Spätstadium der Hefereduktion erhaltenen 96:4- bzw. 5:95-Gemischen von $(5 S, 11 S)$-28 und $(5 R, 11 S)$-29 entnommen. Charakteristisch sind die Resonanzen der Methinprotonen. Sie liegen bei $\delta=3.74$ (dd, J = 8, $7 \mathrm{~Hz}$ ) [(5S,11S)-28] (Abb. 51) und $\delta=3.85$ (dd, $\mathrm{J}=7.5,7.5 \mathrm{~Hz}$ ) [(5R,11S)-29] (Abb. 52). Die Resonanzen der zughörigen Kohlenstoffatome finden sich $\delta=72.08$ [(5S,11S)-28] (Abb. 92) und 72.84 [(5R,11S)-29] (Abb. 93).

\subsection{Synthese von (5R)-Trispiro[3.0.0.4.2.2]tetradecan [(5R)-13] und (5S)- Trispiro[3.0.0.4.3.2]pentadecan [(5S)-14]}

Nachdem (5R)-24 enantiomerenrein verfügbar war, mussten zur Synthese der Trispirane (5R)-13 und (5S)-14 nur die für die Racemate ausgearbeiteten Synthesen (vgl. 1.3) wiederholt werden. Dies gelang problemlos. Direkte Reduktion von (5R)-24 lieferte (5R)-13, vorherige Ringerweiterung und anschließende Reduktion (5S)-14 (Schema 23). Zur Bestimmung der Drehwerte wurden (5R)-13 $\left(>99 \%\right.$ ee, $\left.[\alpha]_{\mathrm{D}}{ }^{20}=-4.1, \mathrm{c}=1.19, \mathrm{CHCl}_{3}\right)$ und $(5 S)-\mathbf{1 4}$ $\left(>99 \%\right.$ ee, $\left.[\alpha]_{\mathrm{D}}{ }^{20}=-0.9, \mathrm{c}=1.22, \mathrm{CHCl}_{3}\right)$ gaschromatographisch gereinigt.

\section{Schema 23}

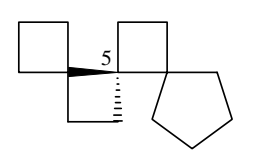

(5R)-13

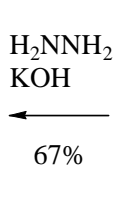

$7 \%$

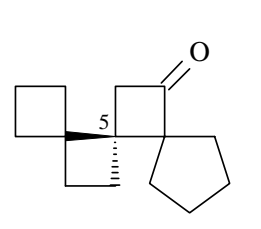

(5R)-24

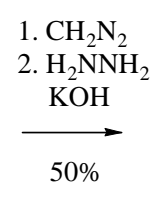

$50 \%$

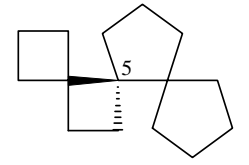

(5S)-14 


\section{Synthesen von (5R*)-Trispiro[3.0.0.4.3.3]hexadecan [(5R*)-15] und rac- Trispiro[4.0.0.4.3.3]heptadecan (rac-8)}

Nachdem sich der Plan einer Synthese der Trispirane 15 und 8 über eine [2+2]Cycloaddition der von 20 bzw. 23 abgeleiteten Keteniminiumsalze 63 und 64 an 1-Methylenspiro[4.4]nonan (25) als nicht realisierbar erwiesen hatte (vgl. 1.2), hielten wir es für geboten, die Zahl der zu anellierenden Ringe auf einen zu beschränken und von einem fertigen Dispiran auszugehen.

\subsection{Synthesen über Dispiro[4.0.4.3]tridecan-1-on (30)}

Als kürzester Weg bot sich an, das bekannte Dispiroketon $30^{23}$ über eine Reaktionsfolge aus Methylenierung, Dichlorketen-Addition und reduktiver Enthalogenierung in das Trispiroketon 34 zu überführen (30-33-34), und dieses dann direkt (34-15) bzw. nach Ringerweiterung mit Diazomethan zu deoxigenieren [34-35(36)-8] (Schema 24). Dass bei der Ringerweiterung von 34 mit 35 und 36 zwei Stereoisomere auftreten konnten, schien wenig störend, da beide Ketone bei der nachfolgenden Deoxigenierung jeweils 8 als alleiniges Produkt liefern mussten.

\section{Schema 24}

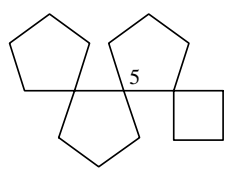

15

$$
\underset{\mathrm{KOH}}{\mathrm{H}_{2} \mathrm{NNH}_{2}} \uparrow
$$

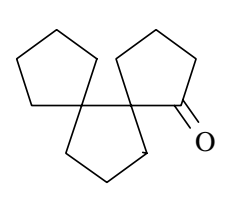

30

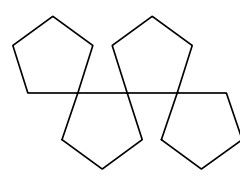

8

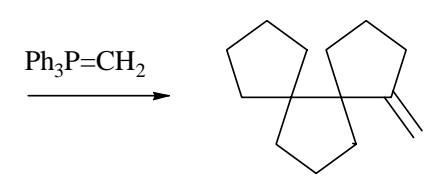

33
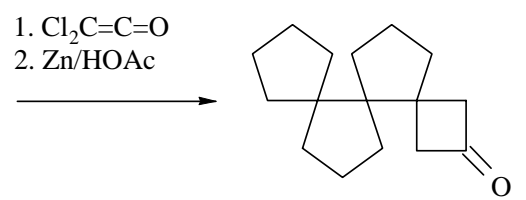

34

$$
\mathrm{CH}_{2} \mathrm{~N}_{2}
$$
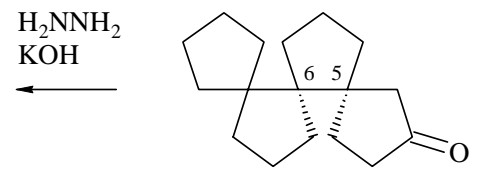

$\left(5 R^{*}, 6 R^{*}\right)-35$

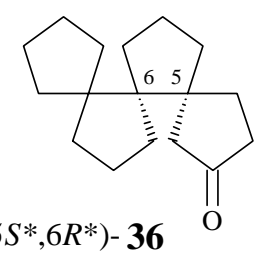




\subsubsection{Ein neuer Zugang zu Dispiro[4.0.4.3]tridecan-1-on (30): Synthese und Umlagerung von Dispiro[3.1.4.3]tridecan-5-on (31)}

Dispiro[4.0.4.3]tridecan-1-on (30) ist erstmals 1996 von Kakiuchi und Mitarbeitern ${ }^{23}$ durch säurekatalysierte Isomerisierung des Cyclopentyliden-cyclooctanons 83 erhalten worden. Dieses wiederum wurde über fünf Stufen aus Cyclooctandiol-1,5 (80) dargestellt (80$\mathbf{8 1}^{58}$-82-83) (Schema 25). Leider lagen alle Ansätze im mmol-Bereich. Es schien daher notwendig, nach einer ergiebigeren Alternative zu suchen.

\section{Schema 25}<smiles>OC1CCCC(O)CCC1</smiles>

80
1. TBDMSCl/Et ${ }_{3} \mathrm{~N}$

2. PCC

$56 \%$

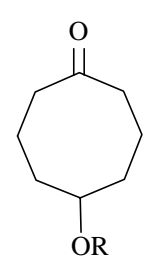

$81 \mathrm{R}=$ TBDMS<smiles>OC1CCCC(=C2CCCC2)CCC1</smiles>

$82 \mathrm{R}=$ TBDMS
1. HF

2. Swern-

Oxidation

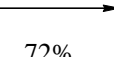

$72 \%$

\%

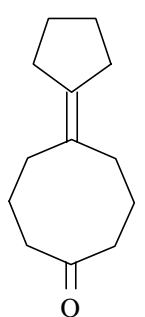

83

$\mathrm{H}_{2} \mathrm{SO}_{4} \downarrow 81 \%$

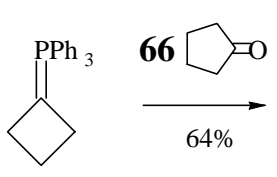

84

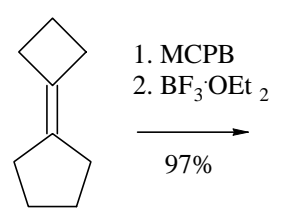

69

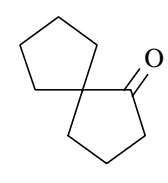

71

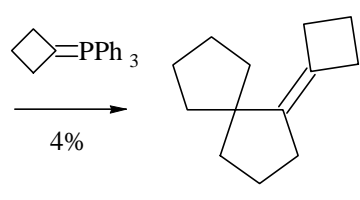

85

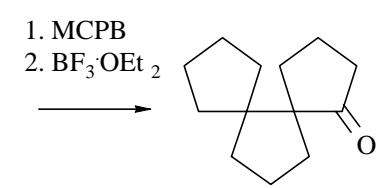

30

$\mathrm{O}_{2} \downarrow 75 \% \quad \mathrm{RSO}_{2} \mathrm{~N}_{3} \downarrow 69 \%$

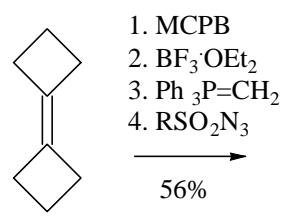

86

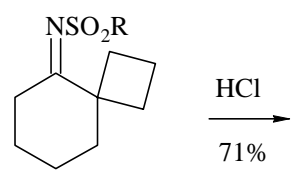

$87 \mathrm{R}=4-\mathrm{NO}_{2} \mathrm{C}_{6} \mathrm{H}_{4}$<smiles>O=C1CCCCC12CCC2</smiles>

32

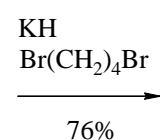$$
76 \%
$$

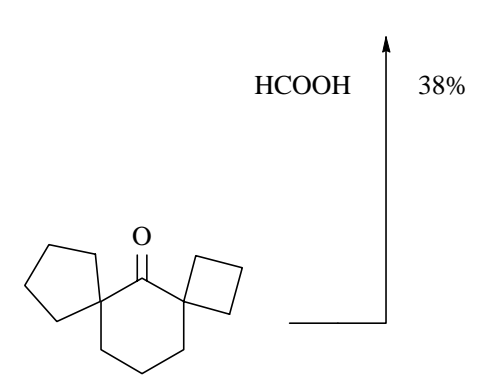

31

Als erste und operativ einfachste Möglichkeit bot sich an, die bei Cyclopentanon (66) problemlose Cyclobutylidenierung mit anschließender Epoxidierung und OxaspirohexanCyclopentanon-Umlagerung (84-69-71) (vgl. 1.1) zu wiederholen (71-85-30) (Schema 25). Leider erwies sich $\mathbf{7 1}$ gegenüber Cyclobutyliden-triphenylphosphoran ${ }^{52}$ als nahezu inert und lieferte lediglich analytische Mengen 85. Symmetriebedingt zeigt das ${ }^{13} \mathrm{C}-\mathrm{NMR}$-Spektrum 
von 85 (Abb. 123) lediglich elf Linien für acht sekundäre und drei quärtäre Kohlenstoffatome. Darunter befinden sich charakteristische Resonanzen für die periphere Methylengruppe des Cyclobutanringes $[\delta=17.75(\mathrm{t})]$, das Spirozentrum $[\delta=52.73(\mathrm{~s})]$ und die Doppelbindung $[\delta$ $=128.94(\mathrm{~s})$ und $140.03(\mathrm{~s})]$.

Als zweite Möglichkeit einer Synthese von 30 wurde eine säurekatalysierte Umlagerung des bisher unbekannten Dispiroketons 31 in Erwägung gezogen. 31 schien durch Spiroalkylierung des Ketons 32 leicht zugänglich und wegen des hohen thermodynamischen Gefälles sukzessiver $\mathrm{C}_{4}-\mathrm{C}_{5}$ - und $\mathrm{C}_{6}-\mathrm{C}_{5}$-Umlagerungen ${ }^{59}$ für eine Umlagerung zu 30 prädestiniert. Das Keton 32 wiederum war in einer früheren Arbeit ${ }^{26}$ aus Bicyclobutyliden (86) ${ }^{52}$ über eine Folge aus Epoxidierung, Oxaspiropentan-Cyclopentanon-Umlagerung, Methylenierung, Umsetzung mit 4-Nitrobenzolsulfonsäureazid und saure Verseifung des resultierenden Sulfonimids 87 dargestellt worden (86-87-32). 87 schien allerdings auch auf einem wesentlich kürzeren Weg über Cyclobutyliden-cyclopentan (69) darstellbar (Schema 25). Ausgehend von 69 umfasste die geplante Synthese von 30 vier Schritte (69-87-32-31-30). Drei davon waren unbekannt.

Erhitzte man 69 mit 4-Nitrobenzolsulfonsäureazid in Acetonitril 18 h unter Rückfluss, so isolierte man das erwünschte Sulfonimid 87 in einer Ausbeute von 69\%. Dies macht deutlich, dass die 1,3-dipolare Cycloaddition von 4-Nitrobenzolsulfonsäureazid an 69 wie erwünscht regioselektiv über das Zwitterion $\mathbf{8 8}$ und das $\Delta^{2}$-Triazolin 89 abgelaufen war (Schema 26). ${ }^{60-63}$ Ein Grund hierfür könnte in der höheren Stabilität von Cyclopentylgegenüber Cyclobutyl-Kationen liegen.

\section{Schema 26}

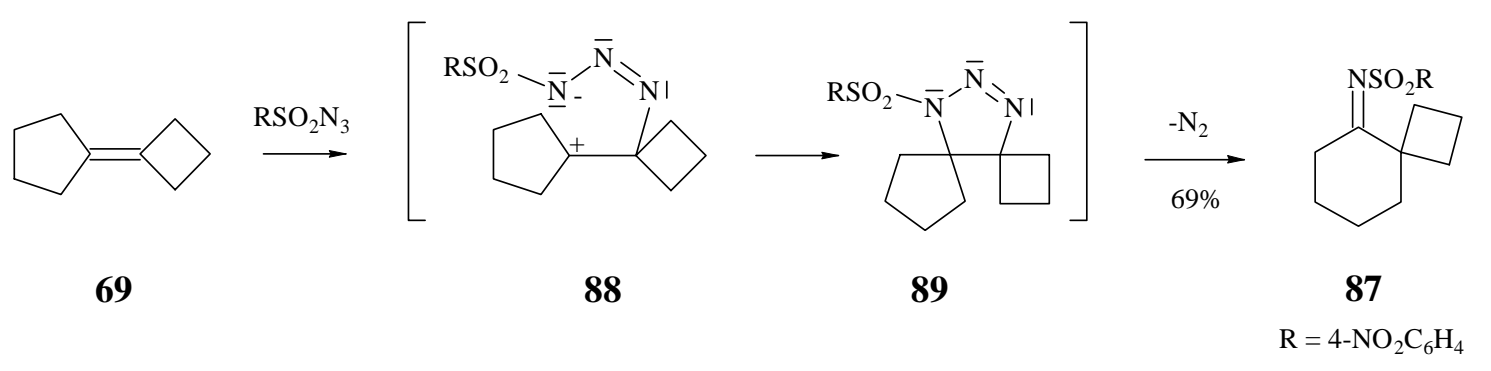

Die Hydrolyse von 87 wurde wie in der Literatur angegeben ${ }^{26}$ durchgeführt und das gebildete Spiro[3.5]nonan-5-on (32) anschließend cycloalkyliert. Hier war eine Analogreaktion mit Spiro[4.4]nonan-1-on (71) bekannt. ${ }^{64}$ Behandelte man 32 in Gegenwart eines Überschusses an Kaliumhydrid mit 1,4-Dibrombutan, so war die Cycloalkylierung nach $90 \mathrm{~h}$ bei Raumtemperatur komplett. Aufarbeitung und Destillation lieferten 31 in einer isolierten Ausbeute von 76\% (Schema 27). Der Erfolg der Reaktion war am ${ }^{13} \mathrm{C}-\mathrm{NMR}-$ Spektrum von 31 (Symmetrie $\mathrm{C}_{\mathrm{s}}$ ) leicht ablesbar (Abb. 94). Es zeigt die nach Zahl und Multiplizität zu fordernden zehn Resonanzen für sieben sekundäre und drei quartäre Kohlenstoffatome. 


\section{Schema 27}

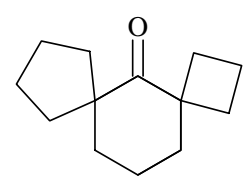

31

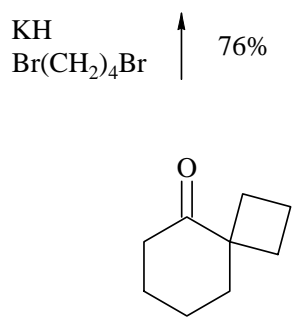

32

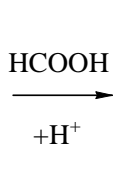

$\mathrm{H}^{+}$

1

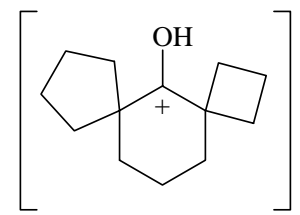

90

$\mathrm{C}_{4}-\mathrm{C}_{5}$<smiles>OC12CCCC1CCCC21CCCC1</smiles>

91

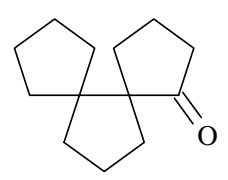

30

$$
+\mathrm{H}^{+} \downarrow \mid-\mathrm{H}^{+}
$$

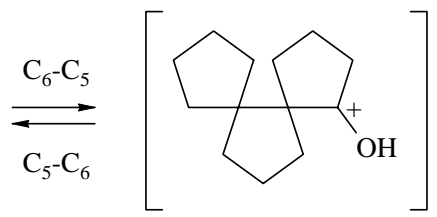

92

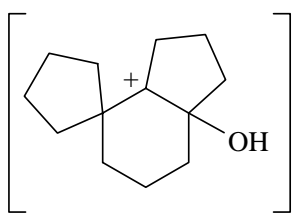

93

Die abschließende Umlagerung von 31 erwies sich als problematisch. Nach Fehlversuchen mit 0.74 M p-Toluolsulfonsäure in Benzol, 5\% Schwefelsäure auf Kieselgel in Hexan sowie mit Nafion ${ }^{\circledR}$ SAC 13 in Benzol, führte reine Ameisensäure zu einem Teilerfolg. Hielt man eine Lösung von 31 in Ameisensäure 26 h bei $50-55^{\circ} \mathrm{C}$, so bildeten sich zwar schwarze Polymere, im niedermolekularen Anteil war jedoch 30 das Hauptprodukt. Aufarbeitung und Chromatographie lieferten es in einer Reinausbeute von 38\% (Schema 27). Seine ${ }^{1} \mathrm{H}$ - und ${ }^{13} \mathrm{C}$ NMR-Daten stimmten mit Literaturdaten ${ }^{23}$ überein.

Mechanistisch führt der Weg zu 30 über die Carbeniumionen 90, 91 und 92. Für die beobachtete Bildung von Polymeren kommen nur 91 und/oder 93 bzw. davon ableitbare Produkte in Frage. Wahrscheinlich können sie nicht nur direkt, sondern auch nach Bildung von 30 durch Reprotonierung und $\mathrm{C}_{5}-\mathrm{C}_{6}$-Ringerweiterung entstehen [30-92-91(93)]. Präparativ ist die Umlagerung zu 30 nur bedingt brauchbar. Gleichwohl erlaubte sie, den Plan einer Methylenierung mit nachfolgender [2+2]-Cycloaddition von Dichlorketen zu prüfen. 


\subsubsection{Versuchte Cycloaddition von Dichlorketen an 1-Methylendispiro[3.0.4.3]- tridecan (33)}

Nachdem ein präparativ gangbarer Weg zu 30 gefunden war, sollte dieses zunächst zu 33 methyleniert und anschließend mit Dichlorketen umgesetzt werden. Geplant war, das als [2+2]Cycloaddukt erhoffte Dichlorcyclobutanon nach reduktiver Enthalogenierung zu 34 sowohl direkt, als auch nach vorheriger Ringerweiterung zu deoxigenieren. Auf diese Weise wären vier- bzw. fünfstufige Synthesen der noch fehlenden Trispirane $\mathbf{1 5}$ und $\mathbf{8}$ realisierbar gewesen (vgl. Schema 24).

Zur Methylenierung ${ }^{36}$ verwendete man mit Kalium-tert-butylat in Ether generiertes Ylid, destillierte den Hauptteil des Lösungsmittels ab und versetzte die zurückbleibende Suspension mit 30. Nach 2.5 h bei $50^{\circ} \mathrm{C}$ war die Umsetzung komplett. Aufarbeitung und Chromatographie lieferten 33 in einer Ausbeute von 81\% (Schema 28). 33 zeigte im ${ }^{1} \mathrm{H}-\mathrm{NMR}$ Spektrum (Abb. 54) unter anderem Resonanzen für die beiden vinylischen Protonen $(\delta=4.80$, ddd, $\mathrm{J}=2.5,1.25,1.25 \mathrm{~Hz}, 1 \mathrm{H}$ und 5.04, ddd, $\mathrm{J}=2.5,1.25,1.25 \mathrm{~Hz}, 1 \mathrm{H})$, und im ${ }^{13} \mathrm{C}-\mathrm{NMR}-$ Spektrum (Abb. 95) Resonanzen für eine 1,1-disubstituierte Doppelbindung [ $\delta=106.54$ (t) und $158.15(\mathrm{~s})]$.

\section{Schema 28}

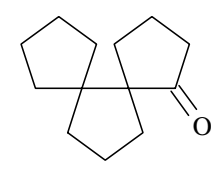

30

$\mathrm{Ph}_{3} \mathrm{P}=\mathrm{CH}_{2} \downarrow 81 \%$

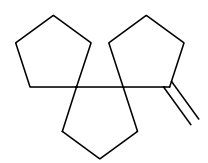

33<smiles>O=C=C(Cl)Cl</smiles><smiles>OC(CC1=CCCC12CCCC2)=C(Cl)Cl</smiles>

96

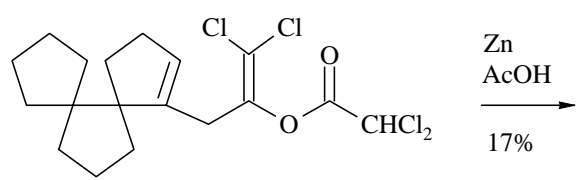

94

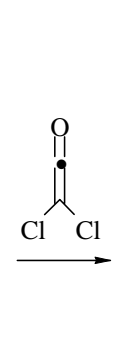

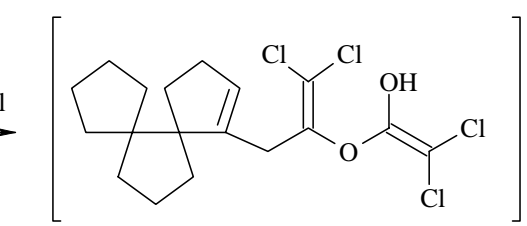

97

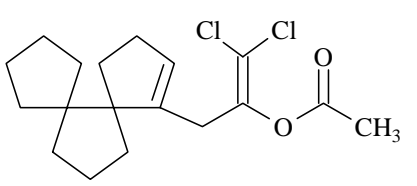

95 
Als nächstes wurde versucht, 33 durch eine [2+2]Cycloaddition mit aus Trichloracetylchlorid und Zink unter Ultraschall generiertem Dichlorketen in ein Dichlorcyclobutanon zu überführen und dieses zu enthalogenieren. ${ }^{24}$ Die Umsetzung mit Dichlorketen lieferte innerhalb $6 \mathrm{~h}$ bei $40^{\circ} \mathrm{C}$ laut GC-Analyse ein Hauptprodukt. Unterwarf man das rohe Gemisch der Umsetzung mit Dichlorketen einer reduktiven Enthalogenierung mit Zink in Eisessig, so isolierte man nach $1 \mathrm{~h}$ bei $60^{\circ} \mathrm{C}$ in $17 \%$ Ausbeute ein Folgeprodukt, das sich nach säulenchromatographischer Reinigung aufgrund seiner spektroskopischen Daten als $\mathbf{9 5}$ zu erkennen gab (Schema 28). So deutete sein ${ }^{13} \mathrm{C}$-NMR-Spektrum (Abb. 125) mit insgesamt 18 Resonanzen auf eine zweifache Addition von Dichlorketen, und mit einer Resonanz bei $\delta=167.38$ (s) auf das Vorliegen eines Esters. Resonanzen bei $\delta=114.77$ (s), 128.73 (d), 141.91 (s) und 146.08 (s) belegten das Vorliegen einer tri- und einer tetrasubstituierten Doppelbindung, und Resonanzen bei $\delta=57.25$ (s) und 62.27 (s) den Erhalt der Dispiran-Teilstruktur. In Übereinstimmung mit der angegebenen Struktur zeigte das ${ }^{1} \mathrm{H}-\mathrm{NMR}-$ Spektrum (Abb. 83) Resonanzen für ein vinylisches Proton ( $\delta=5.55$, dddd, $J=1.5,1.5,1.5,1.5 \mathrm{~Hz}, 1 \mathrm{H}$ ) sowie für zwei diastereotope Protonen einer doppelt allylständigen Methylengruppe ( $\delta=3.14$, dddd. $\mathrm{J}=$ 17, 1.5, 1.5, $1.5 \mathrm{~Hz}, 1 \mathrm{H}$ und 3.23, dddd, $\mathrm{J}=17,1.5,1.5,1.5 \mathrm{~Hz}, 1 \mathrm{H}$ ). Aus den übrigen Resonanzen folgte das Vorliegen einer Acetyl-Methylgruppe ( $\delta=2.15$, s, 3H).

Wie die Bildung von 95 zeigt, reagierte 33 mit Dichlorketen nicht, wie erwünscht, unter Cycloaddition, sondern unter zweifacher Addition über 96 und 97 zu 94 . Die anschließende reduktive Enthalogenierung betraf die aktivierte Dichloracetylgruppe, nicht jedoch die 1,1-Dichlorvinylgruppe und führte zu 95. Dieses Ergebnis steht im Einklang mit dem Ergebnis der versuchten Cycloaddition des Keteniminiumsalzes 64 an 1-Methylenspiro[4.4]nonan (25), bei der mit 76 ebenfalls ein offenkettiges Addukt gebildet worden war (vgl. 1.2 und Schema 20).

\subsubsection{Sequentielle Transformation von 30 in $\left(4 R^{*}, 5 S^{*}\right)$-Trispiro[3.0.0.4.3.3]

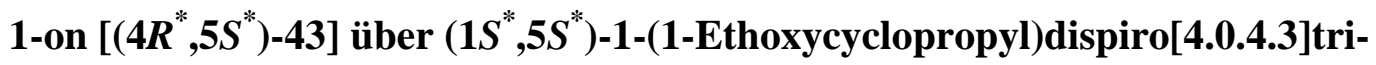 decan-1-ol $\left[\left(1 S^{*}, 5 S^{*}\right)-42\right]$ : Synthese von $\left(5 S^{*}\right)-15$}

Nachdem der Versuch einer Cycloadition von Dichlorketen an 33 gescheitert war, haben wir nach weiteren Möglichkeiten gesucht, das Dispiroketon 30 durch Spiroanellierung eines Cyclobutanons in einen zur Synthese der Trispirane (5S*)-15 und $\mathbf{8}$ geeigneten Vorläufer zu überführen. Leider reagierte $\mathbf{3 0}$ mit den klassischen Spiroanellierungsreagenzien Cyclopropyliden-triphenylphosphoran, ${ }^{32-34}$ Diphenylsulfoniumcyclopropylid ${ }^{27-29}$ und 1-Lithiocyclopropylphenylsulfid $^{30,31}$ nicht. Ein möglicher Grund ist die hohe Enolisierungstendenz von Fünfringketonen. 
Nach diesen Ergebnissen war klar, dass wir versuchen mussten, eine Enolisierung von 30 durch Komplexierung mit wasserfreiem Certrichlorid zu unterdrücken, ${ }^{65}$ und den Komplex anschließend mit einem möglichst „harten“ Nucleophil umzusetzen. Auf der Suche nach einem geeigneten Reagenz stießen wir auf eine Arbeit von Baldwin und Mitarbeitern, ${ }^{35}$ nach der Cyclohexanon (37) durch Addition von 1-Methoxy-vinyllithium, Cyclopropanierung und säurekatalysierte Umlagerung in Spiro[3.5]nonan-1-on (40) überführt werden kann (37-38-3940) (Schema 29). Eine Übertragung dieser Sequenz auf das Dispiroketon 30 mit dem Ziel einer Anellierung eines Cyclobutanons schien aussichtsreich. Ein bzw. zwei Folgeschritte hätten anschließend die gewünschten Trispirane (5S*)-15 und 8 liefern können.

\section{Schema 29}

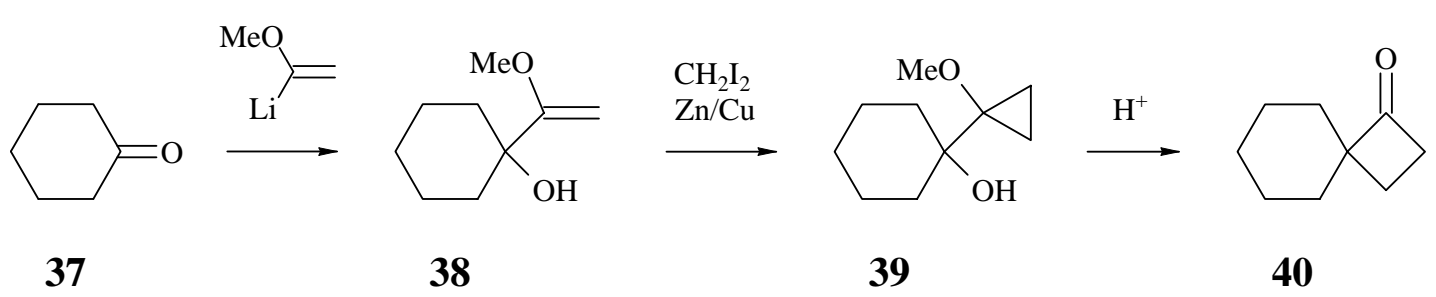

Zur Realisierung der einleitenden Addition verwendeten wir 3.5 Moläquivalente 1Ethoxy-vinyllithium, das durch Metallierung von Ethylvinylether in Tetrahydrofuran mit tertButyllithium bei $-78^{\circ} \mathrm{C}$ leicht zugänglich ist. ${ }^{66}$ Das Reagenz ist bei $0^{\circ} \mathrm{C}$ einige Zeit stabil und wurde bei dieser Temperatur zu mit 2.5 Moläquivalenten wasserfreiem Certrichlorid in Tetrahydrofuran komplexiertem 30 getropft. Anschließend ließ man erwärmen, und nach 45 Min bei Raumtemperatur war der überwiegende Teil des Ketons verbraucht. Hydrolyse und Aufarbeitung lieferten in 94\% Ausbeute ein Rohprodukt, das laut GC 81\% 41 enthielt und als solches zur Cyclopropanierung eingesetzt wurde (Schema 30). Zur Aufnahme der Spektren wurde eine Probe präparativ gaschromatographisch abgetrennt. Wie das ${ }^{1} \mathrm{H}$ - (Abb. 56) und ${ }^{13} \mathrm{C}-\mathrm{NMR}-$ Spektrum (Abb. 97) zeigt, handelt es sich bei $\mathbf{4 1}$ um ein einheitliches Diastereomer. Wir nehmen an, das es durch Addition von 1-Ethoxy-vinyllithium an die sterisch weniger gehinderten Seite von 30 entstanden ist und formulieren es als $\left(1 S^{*}, 5 S^{*}\right)-\mathbf{4 1}$. Der neu eingeführte Rest ist im ${ }^{1} \mathrm{H}-\mathrm{NMR}-\mathrm{Spektrum}$ durch Resonanzen für die Protonen der Ethoxygruppe ( $\delta=0.97, \mathrm{t}, \mathrm{J}=7 \mathrm{~Hz}, 3 \mathrm{H}$ und 3.32, q, $\mathrm{J}=7 \mathrm{~Hz}, 2 \mathrm{H})$ und der Vinylgruppe $(\delta=$ 3.93, d, $\mathrm{J}=2 \mathrm{~Hz}, 1 \mathrm{H}$ und 4.40, d, $\mathrm{J}=2 \mathrm{~Hz}, 1 \mathrm{H}$ ) leicht zu erkennen.

Die Cyclopropanierung von $\left(1 S^{*}, 5 S^{*}\right)-41$ nach einer von Conia ${ }^{67}$ entwickelten Variante der Simmons-Smith-Reaktion war nach 1.5 h komplett. Hydrolyse und Aufarbeitung lieferten in 69\% Ausbeute ein Rohprodukt, das laut GC 80\% 42 enthielt und ohne Reinigung weiter umgesetzt wurde. Da bei der Cyclopropanierung keine Stereozentren angegriffen werden, formulieren wir das Produkt als (1S*,5S*)-42 (Schema 30). Zur Aufnahme der Spektren 
wurde eine Probe präparativ gaschromatographisch gereinigt. Charakteristisch für die ${ }^{1} \mathrm{H}$ - und ${ }^{13} \mathrm{C}$-NMR-Spektren von Cyclopropanen sind Resonanzen bei sehr hohem Feld. Im ${ }^{1} \mathrm{H}$ - (Abb. 57 ) und ${ }^{13} \mathrm{C}-\mathrm{NMR}-S p e k t r u m$ von $\left(1 S^{*}, 5 S^{*}\right)-42$ (Abb. 98) liegen sie bei $\delta=0.62-0.68(\mathrm{~m}, 1 \mathrm{H})$ und 0.67-0.76 (m, 3H) für ${ }^{1} \mathrm{H}$, sowie bei $\delta=8.89(\mathrm{t})$ und $10.37(\mathrm{t})$ für ${ }^{13} \mathrm{C}$. Im Gegensatz zu $\left(1 S^{*}, 5 S^{*}\right)-41$ fallen die Resonanzen der diastereotopen Protonen der Methylengruppe des Ethoxylrestes nicht mehr zusammen. Sie liegen jetzt deutlich separiert bei $\delta=3.13$ (dq, $\mathrm{J}=9$, $7 \mathrm{~Hz}, 1 \mathrm{H}$ ) und 3.35 (dq, J = 9, 7 Hz, 1H). Die Resonanz der Methylprotonen als Kopplungspartner findet sich bei $\delta=0.98$ (dd, $\mathrm{J}=7,7 \mathrm{~Hz}, 3 \mathrm{H}$ ).

\section{Schema 30}

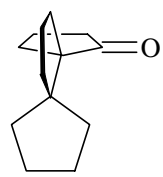

30

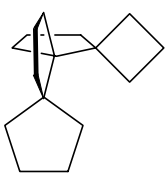

15
2. $\mathrm{Li}-\mathrm{CeCl}_{3} / \mathrm{THF}$

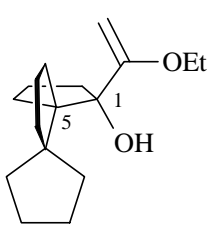

$\left(1 S^{*}, 5 S^{*}\right)-\mathbf{4 1}$

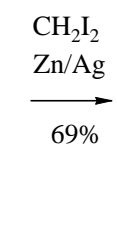

$\left(1 S^{*}, 5 S^{*}\right)-42$

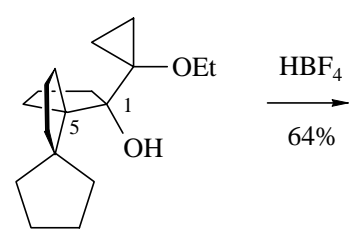

$\left(4 R^{*}, 5 S^{*}\right)-43$ 2,4- $\left(\mathrm{NO}_{2}\right)_{2} \mathrm{C}_{6} \mathrm{H}_{3} \mathrm{NHNH}_{2}$

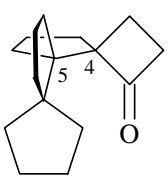

$\overrightarrow{63 \%}$

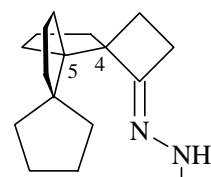

$\left(4 R^{*}, 5 S^{*}\right)-98$

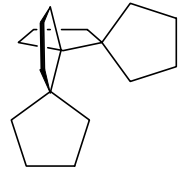

8

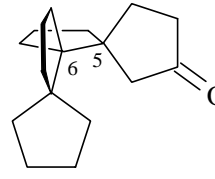

$\left(5 S^{*}, 6 S^{*}\right)-35$

$$
\mathrm{CH}_{2} \mathrm{~N}_{2}=
$$

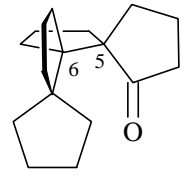

$\left(5 R^{*}, 6 S^{*}\right)-45$

Zur Umlagerung wurde das Rohprodukt der Cyclopropanierung in Ether gelöst und mit 4 Moläquivalenten 48-proz. Tetrafluorborsäure 2 h bei Raumtemperatur gerührt. Aufarbeitung und Chromatographie lieferten in 64\% Ausbeute ein stereochemisch einheitliches Keton, bei dem es sich nach Ausweis seines IR-Spektrums (Abb. 21) mit einer Carbonylfrequenz bei $1770 \mathrm{~cm}^{-1}$ um ein Cyclobutanon handelte. Charakteristisch im ${ }^{1} \mathrm{H}-\mathrm{NMR}$-Spektrum (Abb. 58) war das Verschwinden der Resonanzen der Cyclopropylprotonen und das Auftauchen von drei der vier Resonanzen der Cyclobutylprotonen bei tiefem Feld. Diese lagen bei $\delta$ $=2.25$ (ddd, $\mathrm{J}=10.5,10,7 \mathrm{~Hz}, 1 \mathrm{H}$ ), 2.77 (ddd, $\mathrm{J}=18,10.5,5.5 \mathrm{~Hz}, 1 \mathrm{H}$ ) und 2.88 (ddd, $\mathrm{J}=$ 18, 10, $7 \mathrm{~Hz}, 1 \mathrm{H})$. Ihre Nachbarschaft wurde durch ${ }^{1} \mathrm{~J}_{\mathrm{CH}^{-}}$und ${ }^{3} \mathrm{~J}_{\mathrm{HH}}$-Korrelationsspektren verifiziert. 
Zur Klärung der Frage, um welches von zwei Diastereomeren es sich bei dem gebildeten Keton handelte, haben wir es unter Zusatz von Schwefelsäure mit wässrig-ethanolischem 2,4-Dinitrophenylhydrazin umgesetzt und das gebildete Hydrazon kristallographisch untersucht. Bereits die ungewöhnlich lange Reaktionszeit von sechs Tagen bei Raumtemperatur ließ vermuten, dass es sich bei dem Keton um $\left(4 R^{*}, 5 S^{*}\right)-43$, und damit um das Diastereomer mit endo-ständiger Carbonylgruppe handelte. Diese Vermutung wurde durch die Identifizierung seines 2,4-Dinitrophenylhydrazons als $\left(4 R^{*}, 5 S^{*}\right)-98$ bestätigt (vgl. 5, Abb. 2). Mechanistisch ist der Weg zu $\left(4 R^{*}, 5 S^{*}\right)-43$ klar: er beginnt mit einer stereoselektiven Addition von 1-Ethoxy-vinyllithium an 30 unter Bildung von $\left(1 S^{*}, 5 S^{*}\right)-\mathbf{4 1}$, und endet nach Cyclopropanierung zu $\left(1 S^{*}, 5 S^{*}\right)-42$ mit einer stereoselektiven 1,2-Verschiebung unter Inversion der Konfiguration am Endpunkt der Umlagerung unter Bildung von (4R*,5S*)-43 (Schema 30).

Wie nach der schleppenden Umsetzung mit 2,4-Dinitrophenylhydrazin zu erwarten, war die Deoxigenierung von $\left(4 R^{*}, 5 S^{*}\right)-43$ noch schwieriger als die von 78 (vgl. 1.3). Die dort erfolgreichen Bedingungen, d.h. Erhitzen mit Hydrazinhydrat und gepulvertem $\mathrm{KOH}$ in Diethylenglykol auf $120-200^{\circ} \mathrm{C}$, führten bei $\left(4 R^{*}, 5 S^{*}\right)-43$ zu keiner Reaktion. Erst ein von Barton $^{46}$ für sterisch gehinderte Ketone empfohlener Einsatz von wasserfreiem Hydrazin und dem Natriumalkoholat von Diethylenglykol führte zum Erfolg. Erhitzte man ( $\left.4 R^{*}, 5 S^{*}\right)-\mathbf{4 3}$ mit einem hohen Überschuß dieser Kombination in Diethylenglykol auf $180^{\circ} \mathrm{C}$, so war die Bildung des Hydrazons laut GC-Analyse nach 66 h komplett. Daraufhin wurde die Badtemperatur auf $200-205^{\circ} \mathrm{C}$ erhöht und überschüssiges Hydrazin sowie gebildetes Reaktionswasser unter strömendem Argon weitgehend abdestilliert. Nach weiteren $72 \mathrm{~h}$ bei $200-205^{\circ} \mathrm{C}$ war das Hydrazon verbraucht. Aufarbeitung und Kurzweg-Chromatographie an Kieselgel in Pentan lieferten das erwünschte (5S*)-15 in einer Rohausbeute von 58\% und einer Reinheit von 90\%. Präparative Gaschromatographie ergab eine analysenreine Probe. Das ${ }^{1} \mathrm{H}-\mathrm{NMR}$-Spektrum von (5S*)-15 (Abb. 48) ist im Hochfeldteil wenig charakteristisch. Es zeigt allerdings bei tiefstem Feld eine Serie von drei gut aufgelösten Resonanzen für jeweils ein Proton $(\delta=1.93$, ddd, $\mathrm{J}=$ 12, 8, $4 \mathrm{~Hz}, 1 \mathrm{H}$; 2.25, ddd, J = 10, 10, $10 \mathrm{~Hz}, 1 \mathrm{H}$; 2.31, ddd, J = 10, 10, $10 \mathrm{~Hz}, 1 \mathrm{H}) . \operatorname{Im}{ }^{13} \mathrm{C}-$ NMR-Spektrum (Abb. 89) findet sich erwartungsgemäß der volle Satz von sechzehn Resonanzen, darunter die Resonanzen für das periphere Kohlenstoffatom des Cyclobutans $[\delta$ $=15.91(\mathrm{t})]$ und die Spirozentren [ $\delta=52.08(\mathrm{~s}), 55.73(\mathrm{~s}), 56.49(\mathrm{~s})]$.

Im Gegensatz zu 24 (vgl. 1.3) erwies sich $\left(4 R^{*}, 5 S^{*}\right)-43$ als gegenüber in situ aus pToluolsulfonylmethylnitrosamid (Diazald ${ }^{\circledR}$ ) mit Kaliumhydroxid in wässrig-methanolischer Lösung erzeugtem Diazomethan vollkommen inert. Die als Produkte angestrebten Ketone $\left(5 S^{*}, 6 S^{*}\right)$-35 und $\left(5 R^{*}, 6 S^{*}\right)-45$ (Schema 30) waren damit nicht zugänglich. Um doch noch zu $8 \mathrm{zu}$ gelangen, musste eine Ringerweiterung über eine Oxaspirohexan-CyclobutanonUmlagerung versucht werden. 


\subsubsection{Ringerweiterung von $\left(4 R^{*}, 5 S^{*}\right)-43$ über 1-Oxatetraspiro[2.0.0.0.4.3.3.2]- octadecan [(3S*,4R*,5S*)-46]: Synthese von rac-8}

Nachdem der Versuch einer Ringerweiterung von $\left(4 R^{*}, 5 S^{*}\right)-43$ mit in situ generiertem Diazomethan keinen Umsatz ergeben hatte (vgl. 3.1.3), wurde als Alternative eine von Leriverend und Mitarbeitern ${ }^{37,38}$ beschriebene Methodik einer durch Lithiumiodid ausgelösten Oxaspirohexan-Cyclopentanon-Umlagerung untersucht (Schema 31). Zur Darstellung des benötigten 1-Methylen-trispiro[3.0.0.4.3.3] hexadecan $\left(4 R^{*}, 5 S^{*}\right)-44$ haben wir unsere Methodik zur Methylenierung sterisch gehinderter Ketone ${ }^{36}$ angewandt und $\left(4 R^{*}, 5 S^{*}\right)-\mathbf{4 3}$ mit einem Überschuss an mit Kalium-tert-butylat in Benzol generiertem Methylentriphenylphosphoran umgesetzt. Nach $1.5 \mathrm{~h}$ bei $120^{\circ} \mathrm{C}$ war das Edukt laut GC-Analyse verbraucht. Aufarbeitung und Kurzweg-Chromatographie an Kieselgel in Pentan lieferten das erwünschte (4R*,5S*)-44 in $87 \%$ Ausbeute als farblosen Feststoff mit einem Schmelzintervall von $27-33^{\circ} \mathrm{C}$. Sein ${ }^{1} \mathrm{H}$ NMR-Spektrum (Abb. 59) zeigte die Resonanzen der vinylischen Protonen der exocyclischen Doppelbindung bei $\delta=4.83$ (dd, $\mathrm{J}=2.25,2.25 \mathrm{~Hz}, 1 \mathrm{H}$ ) und 4.85 (dd, $\mathrm{J}=2.25,2.25 \mathrm{~Hz}, 1 \mathrm{H}$ ), und sein ${ }^{13} \mathrm{C}$-NMR-Spektrum (Abb. 100) die Resonanzen der zugehörigen Kohlenstoffatome bei $\delta=106.24(\mathrm{t})$ und $158.57(\mathrm{~s})$.

\section{Schema 31}

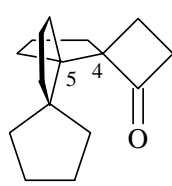

$\left(4 R^{*}, 5 S^{*}\right)-43$

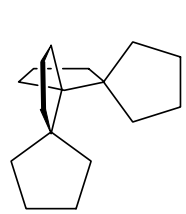

rac- $\mathbf{8}$
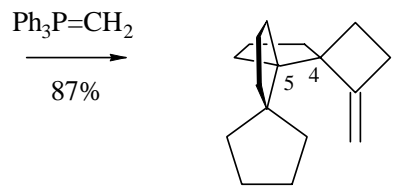

$\left(4 R^{*}, 5 S^{*}\right)-44$

$\left(3 S^{*}, 4 R^{*}, 5 S^{*}\right)-46$
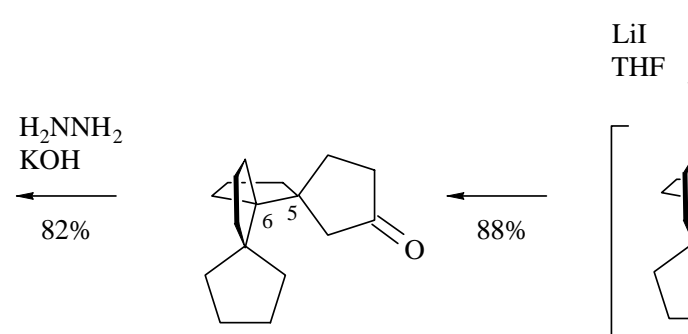

$\left(5 S^{*}, 6 S^{*}\right)-35$

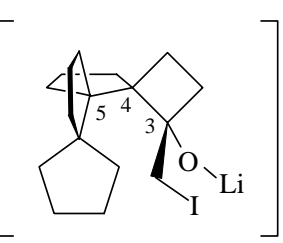

99

Zur Epoxidierung von $\left(4 R^{*} 5 S^{*}\right)-44$ verwendeten wir 3-Chlorperbenzoesäure, wobei mit der Bildung von zwei stereoisomeren Epoxiden zu rechnen war. Wir waren deshalb positiv überrascht, dass nach 1.5 h bei Raumtemperatur dünnschichtchromatographisch nur eine einzige neue Zone zu beobachten war. Aufarbeitung und Chromatographie lieferten in 58\% ein farbloses Epoxid vom Schmelzpunkt $78^{\circ} \mathrm{C}$, das laut ${ }^{1} \mathrm{H}$ - (Abb. 60) und ${ }^{13} \mathrm{C}-\mathrm{NMR}-\mathrm{Spek}$ - 
trum (Abb. 101) stereochemisch einheitlich war. Es lag auf der Hand, dass es sich dabei um $\left(3 S^{*}, 4 R^{*}, 5 S^{*}\right)-\mathbf{4 6}$, und damit um das durch Angriff der Persäure von der sterisch weniger gehinderten Seite von (4R*5S*)-44 gebildete Epoxid handelte. Sein ${ }^{1} \mathrm{H}-\mathrm{NMR}-\mathrm{Spektrum}$ zeigte die Resonanzen der diastereotopen Protonen des Epoxidringes bei $\delta=2.43$ (d, $\mathrm{J}=4.5 \mathrm{~Hz}, 1 \mathrm{H}$ ) und $2.83\left(\mathrm{~d}, \mathrm{~J}=4.5 \mathrm{~Hz}\right.$ ), und sein ${ }^{13} \mathrm{C}$-Spektrum die zugehörige Kohlenstoffresonanz bei $\delta=$ $53.97(\mathrm{t})$.

Behandelte man $\left(3 S^{*}, 4 R^{*}, 5 S^{*}\right)-46$ mit einer äquimolaren Menge Lithiumiodid in siedendem Tetrahydrofuran, so bildete sich innerhalb von $6 \mathrm{~h}$ in 88\% isolierter Ausbeute unter selektiver 1,2-Verschiebung der höher substituierten Bindung das Keton (5S*,6S*)-35 (4699-35) (Schema 31). Seine Struktur folgte aus einer Carbonylfrequenz bei $1745 \mathrm{~cm}^{-1} \mathrm{im}$ IRSpektrum (Abb. 18), aus einer Resonanz für das zugehörige Carbonyl-Kohlenstoffatom bei $\delta$ = 216.71 (s) im ${ }^{13} \mathrm{C}$-Spektrum (Abb. 96), sowie aus einem AB-System für die Protonen der isolierten Methylengruppe bei $\delta=1.96(\mathrm{~d}, \mathrm{~J}=17 \mathrm{~Hz}, 1 \mathrm{H})$ und $2.17(\mathrm{~d}, \mathrm{~J}=17 \mathrm{~Hz}, 1 \mathrm{H}) \mathrm{im}{ }^{1} \mathrm{H}-$ NMR-Spektrum (Abb. 55). Es sei darauf hingewiesen, dass 1-Oxaspiro[2.3] hexane mit Bortrifluorid-Etherat und Lithiumiodid völlig unterschiedlich reagieren: Bortrifluorid-Etherat als Elektrophil öffnet die laterale, Iodid als Nucleophil dagegen die distale Bindung. Entsprechend unterschiedlich sind die Produkte: Bortrifluorid führt zu Aldehyden, Alkoholen und Lactonen, ${ }^{68}$ Lithiumiodid dagegen zu Cyclopentanonen. ${ }^{37-39}$

Die abschließende Wolff-Kishner-Reduktion wurde nach der Variante von HuangMinlon ${ }^{18}$ mit Hydrazinhydrat und gepulvertem $\mathrm{KOH}$ in Diethylenglykol bei $180^{\circ} \mathrm{C}$ durchgeführt. Nach 2 h war die Reaktion beendet. Aufarbeitung und Kurzweg-Chromatographie an Kieselgel in Pentan lieferten das gewünschte Trispiran rac-8 in einer Ausbeute von 82\%. In Übereinstimmung mit seiner Struktur (Symmetrie $\mathrm{C}_{2}$ ) zeigte sein ${ }^{13} \mathrm{C}-\mathrm{NMR}$-Spektrum (Abb. 84) für insgesamt siebzehn Kohlenstoffatome lediglich neun Resonanzen [ $\delta=19.57(\mathrm{t}), 23.39$ $(\mathrm{t}), 24.32(\mathrm{t}), 32.54(\mathrm{t}), 35.21(\mathrm{t}), 35.79(\mathrm{t}), 40.94(\mathrm{t}), 55.92(\mathrm{~s}), 56.65(\mathrm{~s})]$.

In diesem Stadium der Arbeit war klar, dass die racemischen Trispirane 15 und $\mathbf{8}$ in vier bzw. sieben Stufen aus dem Dispiroketon 30 darstellbar waren. Bezüglich der angestrebten enantioselektiven Synthesen fiel allerdings negativ ins Gewicht, dass (a) orientierende Versuche einer Racematspaltung auf der Stufe des Ketons 30 negativ ausgefallen waren, dass (b) eine Racematspaltung auf der Stufe des Ketons $\left(4 R^{*}, 5 S^{*}\right)-43$ wegen mangelnder Reaktivität ohne Erfolgsaussichten schien, und dass (c) die Synthese von 8 mit sieben Schritten zwei Schritte länger war als geplant. Mit der zusätzlichen Erschwernis einer nicht optimalen Synthese von $\mathbf{3 0}$ lag es nahe, effektivere und für enantioselektive Synthesen besser geeignete Wege zu 15 und 8 zu suchen. 


\subsection{Synthesen von rac-15 und rac-8 über Dispiro[3.0.4.3]dodecan-1-on (51)}

Auf der Suche nach Alternativen zu den Synthese von 8 und 15 über $\mathbf{3 0}$ stießen wir auf eine Arbeit von Tu und Mitarbeitern ${ }^{40}$ über eine durch Halogen-Kationen induzierte stereoselektive Umlagerung von Allylalkoholen in $\beta$-Halogenketone. Eines der Beispiele betraf 1-(1-Cyclopentenyl)-cyclobutanol (47), das durch Addition eines aus Chloramin-T mit Zinkbromid generierten Bromkations in $\left(5 R^{*}, 6 R^{*}\right)$-6-Brom-spiro[4.4]nonan-1-on [(5R*,6R*)-48] überging (Schema 32).

\section{Schema 32}

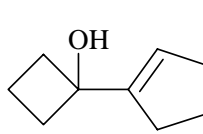

47

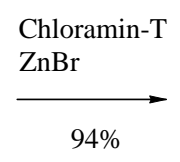

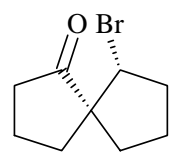

$\left(5 R^{*}, 6 R^{*}\right)-48$

Weitere potentielle Edukte einer Synthese von $\mathbf{8}$ and 15 waren demnach die bisher unbekannten Vinyl-cyclobutanole 52 bzw. 53, die durch Addition von 1-Cyclopentenyl- ${ }^{41}$ bzw. 1-Cyclobutenyllithium ${ }^{42}$ an das ebenfalls unbekannte Dispiro[3.0.4.3]dodecan-1-on (51) zugänglich schienen (Schema 33). Natürlich war uns bewusst, dass bei 52 und 53 jeweils zwei, und, nach erhoffter regioselektiver Umlagerung, bei 54 bzw. 56 sogar vier Diastereomere auftreten konnten. Die stereoselektive Addition von 1-Ethoxy-vinyllithium an $\mathbf{3 0}$ (vgl. 3.1.3) sowie die stereoselektive Umlagerung von 47 ließen uns jedoch hoffen, dass auch bei 52 und 53, sowie bei 54 und 56 jeweils nur ein Diastereomer auftreten würde. Im Erfolgsfall sollten 54 und 56 reduktiv enthalogeniert und anschließend zu 8 bzw. 15 deoxigeniert werden. Im Folgenden beschreiben wir zunächst die Synthese von 51.

\section{Schema 33}

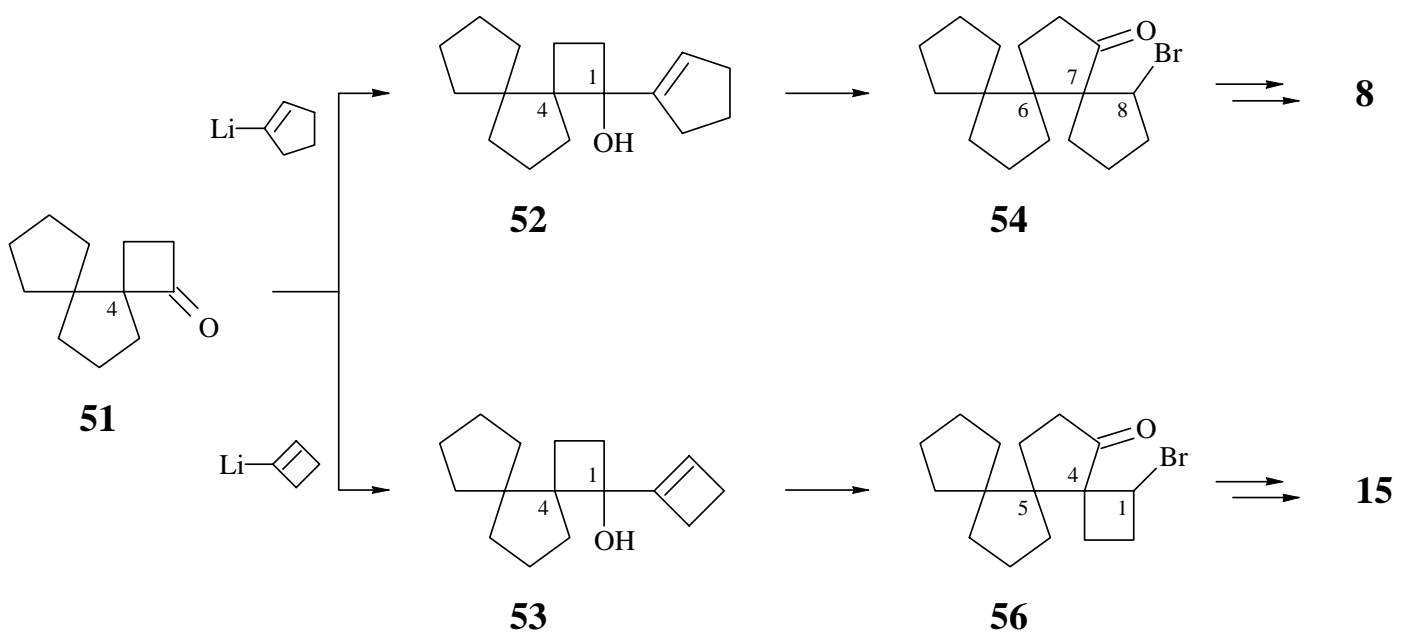




\subsubsection{Synthese von 51}

Zur Synthese von Dispiro[3.0.4.3]dodecan-1-on (51) bot sich an, das nach einem im Rahmen dieser Arbeit entwickelten Verfahren (vgl. 1.1) nunmehr leicht zugängliche Spiroketon 71 über eine Reaktionsfolge aus Cyclopropylidenierung, Epoxidierung und Oxaspiropentan-Cyclobutanon-Umlagerung in 51 zu überführen (71-49-51) (Schema 34).

\section{Schema 34}

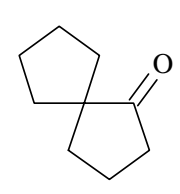

71

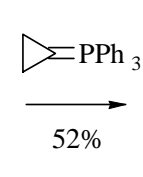

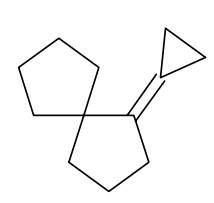

49

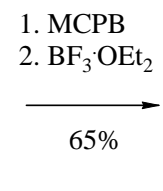

$65 \%$

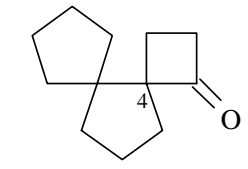

51

Die Cyclopropylidenierung von $\mathbf{7 1}$ wurde mit aus Cyclopropyltriphenylphosphoniumbromid und Natriumhydrid in Tetrahydrofuran generiertem Ylid durchgeführt. ${ }^{33,34}$ Nach 40 h bei $70^{\circ} \mathrm{C}$ lieferten Aufarbeitung und Destillation 49 in einer Ausbeute von 52\%. Verwendete man Kalium-tert-butylat in Benzol, so lag die Ausbeute nach $48 \mathrm{~h}$ bei $60^{\circ} \mathrm{C}$ lediglich bei 13\%. Charakteristisch für 49 (Symmetrie $\mathrm{C}_{\mathrm{s}}$ ) sind im ${ }^{1} \mathrm{H}-\mathrm{NMR}-$ Spektrum (Abb. 61 ) ein $\mathrm{AA}^{\prime} \mathrm{BB}^{\prime}$-System für die Protonen des Dreirings $(\delta=0.91,4 \mathrm{H})$, und im ${ }^{13} \mathrm{C}-\mathrm{NMR}$ Spektrum (Abb. 102) Resonanzen für die Kohlenstoffatome des Dreirings $[\delta=-0.17$ (t) und 2.39 (t)] und der Doppelbindung [ $\delta=108.77$ (s) und 138.94 (s)].

Die Umsetzung von 49 mit 3-Chlorperbenzoesäure ${ }^{32}$ und die anschließende in situUmlagerung des gebildeten Epoxids mit Bortrifluorid-Etherat verliefen problemlos und lieferten das gewünschte 51 in einer Ausbeute von 65\%. 51 zeigte im IR-Spektrum (Abb. 25) mit $1770 \mathrm{~cm}^{-1}$ die für ein Vierringketon zu erwartende hohe Carbonylfrequenz, und im ${ }^{13} \mathrm{C}$ Spektrum (Abb. 103) die Resonanz für das zugehörige Carbonyl-Kohlenstoffatom bei $\delta=$ 216.47 (s). Im ${ }^{1}$ H-NMR-Spektrum (Abb. 62) findet sich der komplette Satz von vier Resonanzen für die Protonen des Vierrings bei $\delta=1.74$ (ddd, $\mathrm{J}=11,10.5,6.5 \mathrm{~Hz}, 1 \mathrm{H}$ ), 1.96 (ddd, $\mathrm{J}=$ 11, 11, $7 \mathrm{~Hz}, 1 \mathrm{H}$ ), 2.69 (ddd, $\mathrm{J}=17.5,11,6.5 \mathrm{~Hz}, 1 \mathrm{H}$ ) und 2.87 (ddd, $\mathrm{J}=17.5,10.5,7 \mathrm{~Hz}$, $1 \mathrm{H})$. 


\subsubsection{Sequentielle Transformation von $\left(4 S^{*}\right)-51$ in $\left(6 S^{*}, 7 S^{*}, 8 R^{*}\right)-8$-Brom-trispiro-} [4.0.0.4.3.3]heptadecan-12-on $\left[\left(6 S^{*}, 7 S^{*}, 8 R^{*}\right)-54\right]$ und $\left(1 R^{*}, 4 S^{*}, 5 S^{*}\right)-1-B r o m-$ trispiro[3.0.0.4.3.3]hexadecan-16-on [(1 $\left.\left.R^{*}, 4 S^{*}, 5 S^{*}\right)-56\right]$ über ein Cyclopentenylbzw. Cyclobutenylcyclobutanol: verkürzte Synthese von rac-8

Nachdem das Dispiroketon (4S*)-51 in ausreichender Menge zur Verfügung stand, haben wir dessen Umwandelbarkeit in rac-8 und (5S*)-15 untersucht. Hierzu sollten die Cyclopentenyl- bzw. Cyclobutenylcyclobutanole 52 und 53 dargestellt, mit positiviertem Brom umgelagert, und die als Produkte erhofften $\beta$-Bromketone 54 bzw. 56 zunächst enthalogeniert und dann deoxigeniert werden (51-52-54-8 bzw. 51-53-56-15) (Schema 33). Im Folgenden beschreiben wir zunächst die Versuche zur Darstellung von 8 (Schema 35).

\section{Schema 35}

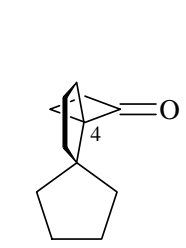

$\left(4 S^{*}\right)-51$

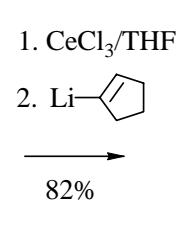

$\left(1 R^{*}, 4 S^{*}\right)-52$
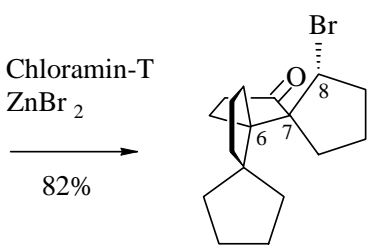

$\left(6 S^{*}, 7 S^{*}, 8 R^{*}\right)-54$

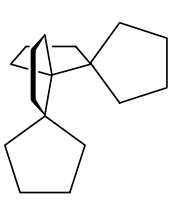

8

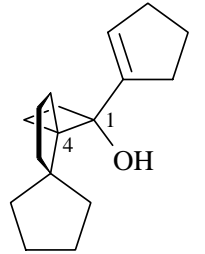

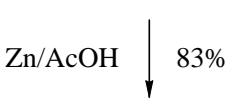
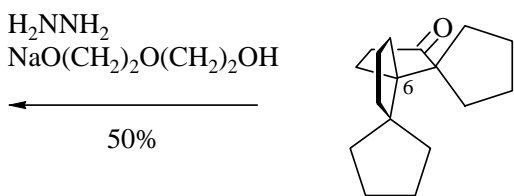

$\left(6 S^{*}\right)-55$

Zur Darstellung von 52 wurde (4S*)-51 zuerst mit zwei Äquivalenten wasserfreiem Certrichlorid in Tetrahydrofuran komplexiert ${ }^{65}$ und anschließend bei $0-20^{\circ} \mathrm{C}$ mit insgesamt 2.4 Äquivalenten einer 0.21 M Lösung von 1-Lithio-cyclopenten in Tetrahydrofuran umgesetzt. Das Reagenz selbst erzeugten wir durch reduktive Metallierung von 1-Brom-cyclopenten mit zwei Äquivalenten tert-Butyllithium. ${ }^{41}$ Aufarbeitung und Chromatographie lieferten in 82\% Ausbeute einen stereochemisch einheitlichen Alkohol, den wir als $\left(1 R^{*}, 4 S^{*}\right)-52$, und damit als durch Angriff des Organometalls von der sterisch weniger gehinderten Seite von 51 entstanden formulieren (Schema 35). Der neu eingeführte Cyclopentenylrest gibt sich im ${ }^{1} \mathrm{H}$ NMR-Spektrum (Abb. 63) durch eine charakteristische Resonanz für ein vinylisches Proton $\left(\delta=5.43\right.$, dddd, $\mathrm{J}=2,2,2,2 \mathrm{~Hz}, 1 \mathrm{H}$ ), und im ${ }^{13} \mathrm{C}$-NMR-Spektrum (Abb. 104) durch Resonanzen für eine trisubstituierte Doppelbindung [ $\delta=125.41$ (d) und 149.40 (s)] zu erkennen. Die Resonanz des die Hydroxylgruppe tragenden Kohlenstoffs liegt bei $\delta=81.24$ (s). 
Zur Umlagerung versetzte man $\left(1 R^{*}, 4 S^{*}\right)$-52 mit einer aus 1.2 Äquivalenten wasserfreiem Zinkbromid und 1.2 Äquivalenten wasserfreiem N-Chlor-p-toluolsulfonsäureamid (Chloramin-T) hergestellten Lösung in Acetonitril. ${ }^{40}$ Nach 15 min bei Raumtemperatur war die Reaktion beendet. Aufarbeitung und Chromatographie lieferten in 82\% Ausbeute ein stereochemisch einheitliches Bromketon als farblosen Feststoff vom Schmp. $76^{\circ} \mathrm{C}$, bei dem es

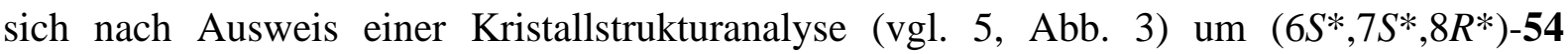
handelte. Demnach war die Umlagerung nicht nur im gewünschten Sinne regioselektiv, sondern auch stereoselektiv abgelaufen und hatte dadurch lediglich eines von acht denkbaren Produkten geliefert. Im ${ }^{1} \mathrm{H}$-NMR-Spektrum (Abb. 65) findet sich unter anderem die Resonanz für das Methinproton deutlich abgesetzt bei tiefem Feld $[\delta=4.11$ (ddd, $\mathrm{J}=8,8,2.5 \mathrm{~Hz}, 1 \mathrm{H}$ )]. Als Bildungsmechanismus nehmen wir an, dass $\left(1 R^{*}, 4 S^{*}\right)-52$ aus der in Schema 35 gezeigten Konformation heraus reagiert, und dass sich das Brom-Kation von der sterisch weniger abgeschirmten Rückseite des Cyclopentenylrestes her nähert und damit die beobachtete 1,2Verschiebung der höher substituierten Bindung auf der Vorderseite provoziert.

Zur Reduktion von $\left(6 S^{*}, 7 S^{*}, 8 R^{*}\right)-54$ haben wir mit Lithiumaluminiumhydrid in Tetrahydrofuran bei $20^{\circ} \mathrm{C}$, Tri-n-butylzinnhydrid in Toluol bei $110^{\circ} \mathrm{C}$ und Zink in Eisessig bei $60^{\circ} \mathrm{C}$ drei Reagenzien getestet. Lithiumaluminiumhydrid reagierte unter Ringöffnung, Tri-nbutylzinnhydrid unter Ringerweiterung, und nur Zink in Eisessig lieferte das erwünschte $\left(6 S^{*}\right)$-55. Wir isolierten dieses Keton in einer Ausbeute von 83\% als farblosen Feststoff vom Schmp. $67-68^{\circ}$ C. Sein IR-Spektrum (Abb. 29) zeigte eine für Fünfringketone charakteristische Carbonylfrequenz bei $1730 \mathrm{~cm}^{-1}$, und sein ${ }^{13} \mathrm{C}-\mathrm{NMR}-$ Spektrum (Abb. 107) die zugehörige Kohlenstoffresonanz bei $\delta=218.95$ (s). Im ${ }^{1} \mathrm{H}$-NMR-Spektrum (Abb. 66) war die Resonanz des Methinprotons von 54 nicht mehr nachweisbar.

Die abschließende Wolff-Kishner-Reduktion von (6S*)-55 wurde nach der Variante von Barton ${ }^{46}$ mit wasserfreiem Hydrazin und dem Natriumalkoholat von Diethylenglykol durchgeführt und erwies sich als extrem schwierig. Laut einer begleitenden GC-Analyse erforderte die Bildung des Hydrazons $48 \mathrm{~h}$ bei $180^{\circ} \mathrm{C}$, und seine Zersetzung 10 Tage bei 200 $205^{\circ} \mathrm{C}$. Aufarbeitung und Kurzweg-Chromatographie an Kieselgel in Pentan lieferten anschließend das gewünschte Trispiran rac-8 in einer Ausbeute von $50 \%$. Wie seine ${ }^{1} \mathrm{H}$ - und ${ }^{13}$ C-NMR-Daten zeigten, war es mit dem durch Reduktion von 35 erhaltenen Kohlenwasserstoff (vgl. 3.1.4) identisch.

Nachdem die Addition von Cyclopentenyllithium an $\left(4 S^{*}\right)-51$ zu $\left(1 R^{*}, 4 S^{*}\right)-52$ stereoselektiv, und die nachfolgende Umlagerung zu $\left(6 S^{*}, 7 S^{*}, 8 R^{*}\right)-54$ regio- und stereoselektiv abgelaufen war, und auch das Problem der reduktiven Enthalogenierung zu (6S*)-55 sowie dessen Deoxigenierung zu rac-8 gelöst war (Schema 35), glaubten wir uns für eine analoge Synthese von (5S*)-15 gerüstet (Schema 36). Tatsächlich lieferte die im ersten Schritt notwendige Addition von Cyclobutenyllithium an (4S*)-51 innerhalb 1 h in 64\% Ausbeute 
ein stereochemisch einheitliches Cyclobutenyl-cyclobutanol, das wir, wie schon bei $\left(1 R^{*}, 4 S^{*}\right)-52$, als durch Addition des Organometalls von der sterisch weniger abgeschirmten Seite von (4S*)-51 gebildet ansehen und daher als $\left(1 R^{*}, 4 S^{*}\right)-53$ formulieren. Den Cyclobutenylrest erkennt man im ${ }^{1} \mathrm{H}$-NMR-Spektrum (Abb. 64) an einer Resonanz bei $\delta=5.67\left(\mathrm{~m}_{\mathrm{c}}\right.$, $1 \mathrm{H}$ ) für ein vinylisches Proton, und im ${ }^{13} \mathrm{C}$-NMR-Spektrum (Abb. 105) an Resonanzen bei $\delta=$ 127.86 (d) und 153.71 (s) für eine trisubstituierte Doppelbindung. Die Resonanz des die Hydroxylgruppe tragenden Kohlenstoffs liegt bei $\delta=79.97$ (s).

\section{Schema 36}

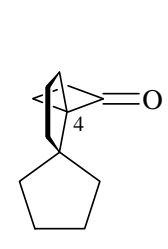

$\left(4 S^{*}\right)-51$

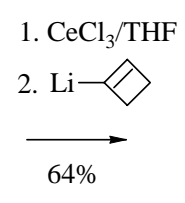

$\left(1 R^{*}, 4 S^{*}\right)-53$

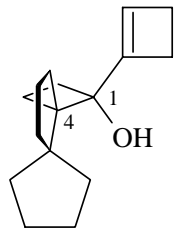

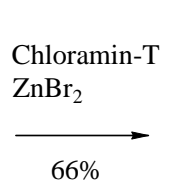

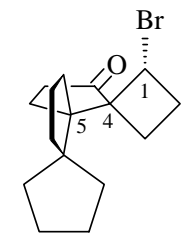

$\left(1 R^{*}, 4 S^{*}, 5 S^{*}\right)-56$

$$
\mathrm{Zn} / \mathrm{AcOH}=
$$

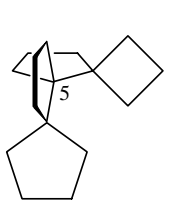

$\left(5 S^{*}\right)-\mathbf{1 5}$

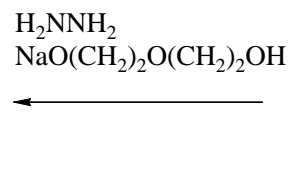

$\left(5 S^{*}\right)-57$

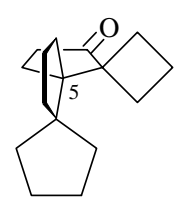

Die Umlagerung von $\left(1 R^{*}, 4 S^{*}\right)-53$ mit Chloramin-T und Zinkbromid in Acetonitril wurde wie bei $\left(1 R^{*}, 4 S^{*}\right)-52$ durchgeführt, war allerdings erst nach $1.5 \mathrm{~h}$ bei Raumtemperatur komplett. Aufarbeitung und Chromatographie lieferten in 66\% Ausbeute ein stereochemisch einheitliches Bromketon vom Schmp. $29^{\circ} \mathrm{C}$, das wir in Analogie zu der durch eine Kristallstruktur gesicherten relativen Konfiguration von $\left(6 S^{*}, 7 S^{*}, 8 R^{*}\right)-54$ als $\left(1 R^{*}, 4 S^{*}, 5 S^{*}\right)-56$ formulieren. Sein IR-Spektrum (Abb. 30) zeigt die Carbonylfrequenz bei $1738 \mathrm{~cm}^{-1}$, und sein ${ }^{13}$ C-NMR-Spektrum (Abb. 108) die Resonanz des zugehörigen Kohlenstoffs bei $\delta=215.24$ (s). Das ${ }^{1} \mathrm{H}-\mathrm{NMR}-$ Spektrum (Abb. 67) zeigt unter anderem eine charakteristische Resonanz für das Methinprotons bei $\delta=4.20(\mathrm{dd}, \mathrm{J}=9,9 \mathrm{~Hz}, 1 \mathrm{H})$.

Leider führten alle Versuche, $\left(1 R^{*}, 4 S^{*}, 5 S^{*}\right)-56$ unter den bei $\left(6 S^{*}, 7 S^{*}, 8 R^{*}\right)-54$ erfolgreichen Bedingungen mit Zink in Eisessig zu enthalogenieren, laut ${ }^{1} \mathrm{H}-\mathrm{NMR}$-Analyse der rohen Reaktionsgemische zu Dienen. Eine Bildung von (5S*)-57 konnte nicht nachgewiesen werden. Auch ein ersatzweise unternommener Versuch einer Eliminierung mit anschließender Hydrierung scheiterte: $\left(1 R^{*}, 4 S^{*}, 5 S^{*}\right)$-56 reagierte mit 1,8-Diazabicyclo[5.4.0]undec7-en (DBU) in Toluol innerhalb $48 \mathrm{~h}$ bei $110^{\circ} \mathrm{C}$ nicht. Damit war klar, dass die Synthese von (5S*)-15 auf einem anderen Weg erfolgen musste. Die von sieben auf vier Schritte verkürzte Synthese von rac-8 dagegen schien optimal. 


\subsubsection{Sequentielle Transformation von (4S*)-51 in Trispiro[3.0.0.4.3.3]hexadecan-16- on (57) über ein $\beta$-Hydroxyselenid: verkürzte Synthese von (5S*)-15}

Nach der fehlgeschlagenen Enthalogenierung von $\left(1 R^{*}, 4 S^{*}, 5 S^{*}\right)-56$ musste ein neuer Weg zu (5S*)-15 gefunden werden. Dabei war wichtig, an (4S*)-51 als gemeinsamem Edukt der Synthesen von rac-8 und (5S*)-15 festzuhalten, um für die spätere Darstellung optisch reiner Vertreter nicht zwei Racemattrennungen durchführen zu müssen. Eine Untersuchung des methodisch attraktivsten und kürzesten Weges von (4S*)-51 zu (5S*)-15 über eine Ringerweiterung eines ß-Hydroxyselenids hatten wir bis zu diesem Zeitpunkt wegen der Giftigkeit selenorganischer Verbindungen und den dadurch notwendigen Schutzmaßnahmen vermieden. Jetzt allerdings sollten entsprechende Versuche durchgeführt werden.

Grundlage waren frühere Arbeiten der Abteilung, ${ }^{43}$ wonach das Trispiroketon 100 nach Addition von 1-Lithio-1-phenylselenocyclobutan oder 1-Lithio-1-methylselenocyclobutan und Oxidation zum Selenon unter spontaner Abspaltung von Phenyl- bzw. Methylseleninsäure eine Ringerweiterung eingeht (100-101-102) (Schema 37). Es schien deshalb wahrscheinlich, dass auch ein von (4S*)-51 abgeleitetes $\beta$-Hydroxyselenid 58 eine solche Reaktion eingehen würde (Schema 37). Unsicherheiten bestanden bezüglich der Effektivität und Stereochemie der Addition, sowie der Regiochemie der Umlagerung. Nachdem in den bisher untersuchten Fällen (vgl. 3.2.2) eine auch bei 58 notwendige 1,2-Verschiebung der höher substituierten Bindung eingetreten war, waren wir optimistisch, dass dies auch bei $\mathbf{5 8}$ geschehen würde. Die abschließende Deoxigenierung zu (5S*)-15 schien unproblematisch.

\section{Schema 37}

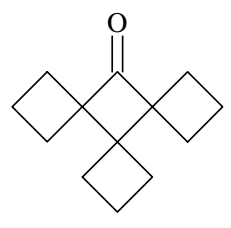

100
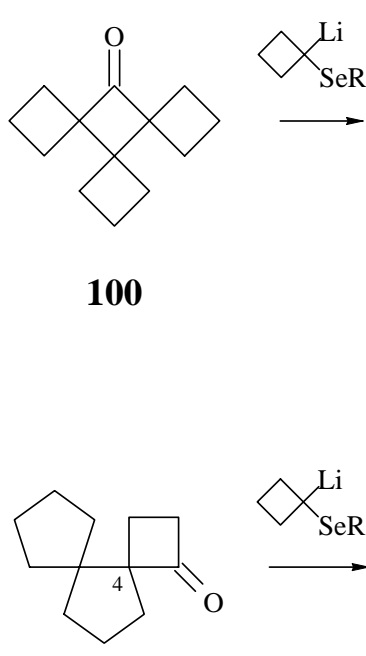

51
101 a $\mathrm{R}=\mathrm{Me}(79 \%)$ b $\mathrm{R}=\mathrm{Ph}(68 \%)$

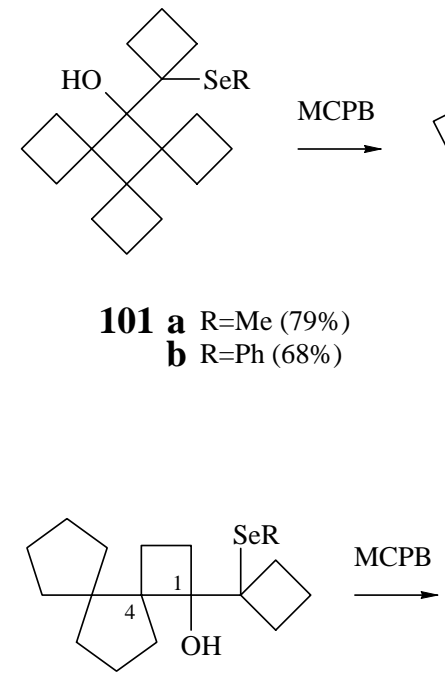

$58 \mathrm{R}=\mathrm{Me}, \mathrm{Ph}$

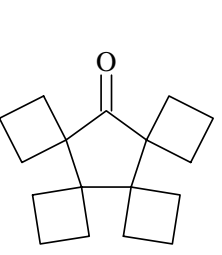

102 aus $\mathrm{R}=\mathrm{Me}(84 \%)$ aus $\mathrm{R}=\mathrm{Ph}(68 \%)$

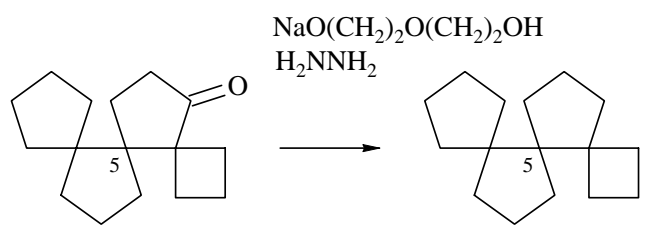

57
15 
Sowohl 1-Lithio-1-phenyl- wie 1-Lithio-1-methylselenocyclobutan können durch reduktive Metallierung der entsprechenden Ketale erzeugt werden. ${ }^{45}$ Diese wiederum sind durch Umsetzung von Cyclobutanon mit Phenyl- bzw. Methylselenol zugänglich, wobei das Reaktionswasser durch Zinkchlorid gebunden werden muss. ${ }^{44}$ In Anbetracht der hohen Flüchtigkeit und Giftigkeit von Methylselenol haben wir zunächst auf eine Darstellung des Dimethylselenoketals verzichtet und in der Abteilung vorhandenes Diphenylselenoketal eingesetzt. Leider gelang eine Addition an (4S*)-51 nach Metallierung nicht. Wir haben daraufhin das Dimethylselenoketal hergestellt und damit den erwünschten Erfolg erzielt.

Tropfte man ( $\left.4 S^{*}\right)-51$ bei $-78^{\circ} \mathrm{C}$ zu einer etherischen Lösung von 1.2 Äquivalenten aus dem Ketal mit tert-Butyllithium erzeugtem 1-Lithio-1-methylselenocyclobutan, so zeigte eine begleitende DC-Analyse nach $1 \mathrm{~h}$ konstante Produktverteilung. Hydrolyse, Aufarbeitung und Chromatographie lieferten in 37\% Ausbeute ein 85:15-Gemisch aus 58 und nicht umgesetztem (4S*)-51, das ohne weitere Reinigung zur Darstellung von (5S*)-57 eingesetzt wurde. Aus dem ${ }^{1} \mathrm{H}$ - (Abb. 69) und ${ }^{13} \mathrm{C}-\mathrm{NMR}-$ Spektrum (Abb. 111) des Gemisches ging hervor, dass das Produkt stereochemisch einheitlich war. Wir sehen es als durch Addition des Reagenzes von der sterisch weniger gehinderten Seite von (4S*)-51 entstanden an und formulieren es als ( $\left.1 S^{*}, 4 S^{*}\right)$-58 (Schema 38). Die Resonanz der Methylprotonen liegt bei $\delta=2.12$ (s, 3H) und die des zugehörigen Kohlenstoffs bei $\delta=4.91$ (q). Die Resonanz des die Hydroxylgruppe tragenden Kohlenstoffs findet sich bei $\delta=83.81$ (s) und die des peripheren Kohlenstoffs des Cyclobutans bei $\delta=16.62$ (t).

\section{Schema 38}

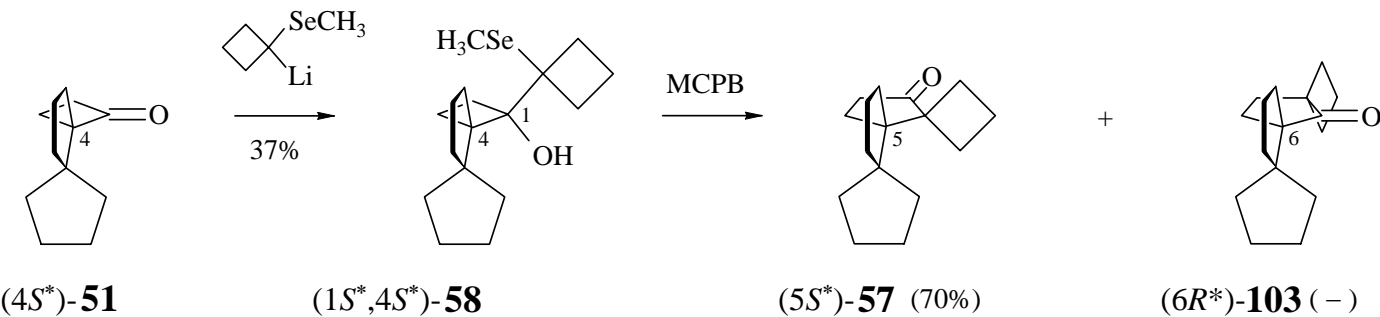

$\left(4 S^{*}\right)-51$

$\left(1 S^{\prime}, 4 S^{*}\right)-58$

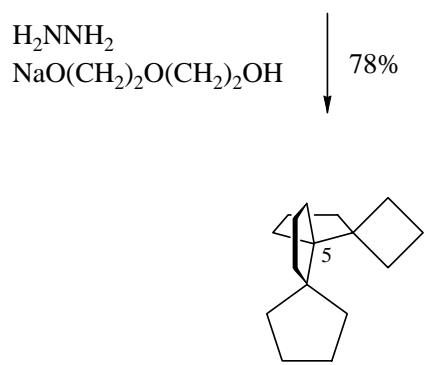

$\left(5 S^{*}\right)-15$
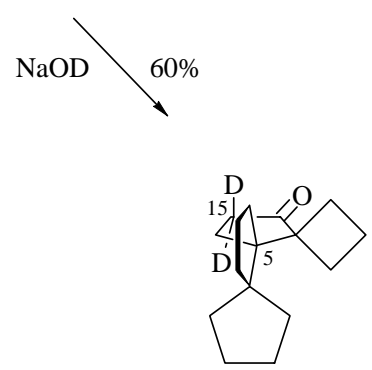

$\left[15,15-\mathrm{D}_{2}\right]-\left(5 S^{*}\right)-57$ 
Um zu gewährleisten, dass $\left(1 S^{*}, 4 S^{*}\right)$-58 möglichst momentan bis zur Stufe des zur Umlagerung befähigten Selenons oxidiert werden würde, haben wir es bei Raumtemperatur mit einem hohen Überschuss (7 Äquivalente) an 3-Chlorperbenzoesäure umgesetzt. Wie eine begleitende DC-Analyse zeigte, war die Reaktion nach 45 min beendet. Aufarbeitung und Chromatographie lieferten in 70\% Ausbeute ein Keton, von dem wir zunächst nicht wussten, ob es sich um (5S*)-57 oder $\left(6 R^{*}\right)$-103 handelte. Beide Ketone haben dieselbe Symmetrie $\left(C_{1}\right)$ und schienen auf der Grundlage spektroskopischer Daten schwer unterscheidbar. Wir haben deshalb einen Entscheid über einen H/D-Austausch gesucht. Hierzu rührten wir das Keton in einem Zweiphasengemisch aus 2N NaOD und Tetrahydrofuran bei Raumtemperatur und beobachteten einen langsamen aber stetigen Anstieg des Deuterierungsgrades. Nach fünfmaliger Erneuerung des NaOD und insgesamt zehn Tagen Reaktionszeit enthielt das Keton $27 \% \mathrm{D}_{1}$ und $73 \% \mathrm{D}_{2}$ (MS). Damit war erwiesen, dass es sich um [15,15- $\left.\mathrm{D}_{2}\right]-\left(S^{*}\right)-\mathbf{5 7}$, und bei dem zugrunde liegenden Keton um (5S*)-57 handelte (Schema 38). Die Umlagerung war demnach wie erwünscht unter 1,2-Verschiebung der höher substituierten Bindung abgelaufen. In Einklang mit der angegebenen Struktur zeigte das ${ }^{13}$ C-NMR-Spektrum von [15,15- $\left.\mathrm{D}_{2}\right]$ (S*)-57 (Abb. 110) bis auf die Resonanz des der Ketogruppe benachbarten sekundären Kohlenstoffatoms bei $\delta=33.38$ den kompletten Liniensatz von (5S*)-57 (Abb. 109). Anstelle der fehlenden Resonanz zeigte das Spektrum bei $\delta=33.02$ ein intensitätsschwaches Triplett ( $\mathrm{J}$ $=20 \mathrm{~Hz}$ ), und bei $\delta=32.71$ ein etwas stärkeres Triplett vom Triplett $(\mathrm{J}=20,20 \mathrm{~Hz})$, wie es für $\mathrm{CD}_{1}$ - bzw. $\mathrm{CD}_{2}$-Fragmente typisch ist.

Wie schon bei $\left(4 R^{*}, 5 S^{*}\right)-43$ (vgl. 3.1.3) und (6S*)-55 (vgl. 3.2.2), erfolgte die abschließende Reduktion von (5S*)-57 nach Wolff-Kishner in der Variante von Barton $^{46}$ mit wasserfreiem Hydrazin und dem Natriumalkoholat von Diethylenglykol. Diesmal erforderte die Bildung des Hydrazons 16 h bei $180^{\circ} \mathrm{C}$, und die nachfolgende Zersetzung drei Tage bei 200-205 ${ }^{\circ}$ C. Aufarbeitung und Reinigung lieferten das erwünschte Trispiran rac-15 in einer Ausbeute von $78 \%$. Seine ${ }^{1} \mathrm{H}$ - und ${ }^{13} \mathrm{C}$-NMR-Daten waren mit denen über $\left(4 R^{*}, 5 S^{*}\right)-43$ dargestellten Materials (vgl. 3.1.1) identisch.

Nachdem, ausgehend von $\left(4 S^{*}\right)-51$, eine vierstufige Synthese von rac-8 und eine dreistufige Synthese von (5S*)-15 verfügbar waren, verblieb als einziges Problem auf dem Weg zu enantiomerenreinen Vertretern das einer Racemattrennung auf der Stufe von (4S*)51. Dieses Problem wurde haben wir sowohl durch Derivatisierung mit (+)-(S)- $N, S$-Dimethyl$S$-phenylsulfoximin ${ }^{47,48}$ wie durch asymmetrische Reduktion mit (-)-Diisopinocampheylchloroboran [(-)-DIP-Cl $]^{15}$ zu lösen versucht. 
Racemattrennung von (4S*)-Dispiro[3.0.4.3]dodecan-1-on [(4S*)-51] mit

$(+)-(S)-N, S$-Dimethyl-S-phenylsulfoximin und (-)-Diisopinocampheylchloroboran: Synthese von (5R)-Trispiro[3.0.0.4.3.3]hexadecan [(5R)-15] und $(P)$-Trispiro[4.0.0.4.3.3]heptadecan $[(P)-8]$

Wie bereits erwähnt, wurden zur Trennung der Enantiomeren von (4S*)-51 zwei Methoden geprüft: (a) eine Derivatisierung mit (+)-(S)-N,S-Dimethyl-S-phenylsulfoximin mit anschließender thermischer Spaltung, und (b) eine asymmetrische Reduktion mit (-)-Diisopinocampheylchloroboran [(-)-DIP-Cl] mit anschließender Rückoxidation. Bei beiden Methoden war eine vollständige Trennbarkeit der als Produkte zu erwartenden diastereomeren $\beta$ Hydroxysulfoximine bzw. Alkohole Vorbedingung für einen Erfolg.

\subsection{Racemattrennung von (4S*)-Dispiro[3.0.4.3]dodecan-1-on [(4S*)-51] mit (+)-(S)-N,S-Dimethyl-S-phenylsulfoximin}

Die Umsetzung racemischer Ketone mit lithiiertem (+)-(S)- $N$,S-Dimethyl-S-phenylsulfoximin führt häufig zu gut kristallisierenden $\beta$-Hydroxysulfoximinen und eröffnet damit die Aussicht auf eine kristallographische Festlegung der absoluten Konfiguration. Nachteilig ist, dass bis zu vier Diastereomere entstehen können. Diastereomerenreinheit vorausgesetzt, liefert die thermische Spaltung enantiomerenreine Ketone.

Zur Derivatisierung von (4S*)-51 wurde (+)-(S)-N,S-Dimethyl-S-phenylsulfoximin ${ }^{47,48}$ bei $0^{\circ} \mathrm{C}$ mit n-Butyllithium in Tetrahydrofuran lithiiert und anschließend bei $-78^{\circ} \mathrm{C}$ zu dem mit wasserfreiem Certrichlorid ${ }^{65}$ in Tetrahydrofuran komplexierten Keton getropft. Nach $1 \mathrm{~h}$ bei $-78^{\circ} \mathrm{C}$ und $1 \mathrm{~h}$ bei Raumtemperatur war die Umsetzung beendet. Aufarbeitung und Chromatographie lieferten zwei diastereomerenreine $\beta$-Hydroxysulfoximine in Ausbeuten von 37 und 24\%, die aus Ethanol/Wasser kristallisiert, und anschließend kristallographisch untersucht wurden (vgl. 5, Abb. 4 und 5). Danach handelte es sich bei dem Majoritätsisomer um (SS,1R,4R)-59 (Schmp. $61-63^{\circ} \mathrm{C} ;[\alpha]_{\mathrm{D}}{ }^{20}=+61.3$, с 1.07, $\mathrm{CHCl}_{3}$ ), und bei dem Minoritätsisomer um (SS,1S,4S)-61 (Schmp. $70^{\circ} \mathrm{C}$; $[\alpha]_{\mathrm{D}}{ }^{20}=+42.0$, с 1.06, $\mathrm{CHCl}_{3}$ ) (Schema 39). Wegen bei Raumtemperatur zu beobachtender Linienverbreiterungen durch dynamische Effekte wurden die ${ }^{1} \mathrm{H}-\mathrm{NMR}$-Spektren beider Sulfoximine bei $-50^{\circ} \mathrm{C}$ aufgenommen. Bei dieser Temperatur waren die AB-Systeme der diastereotopen Protonen der dem Schwefel benachbarten Methylengruppe gut aufgelöst und lagen bei $\delta=3.22\left(\Delta \mathrm{v}_{\mathrm{AB}}=45 \mathrm{~Hz}, \mathrm{~J}_{\mathrm{AB}}=13\right.$ $\mathrm{Hz}, 2 \mathrm{H})$ in (SS,1R,4R)-59 (Abb. 70), und $\delta=3.33\left(\Delta \mathrm{v}_{\mathrm{AB}}=29 \mathrm{~Hz}, \mathrm{~J}_{\mathrm{AB}}=14 \mathrm{~Hz}, 2 \mathrm{H}\right)$ in (SS,1S,4S)-61 (Abb. 72). 


\section{Schema 39}

(-)-(SS)- $\mathrm{C}_{6} \mathrm{H}_{5} \mathrm{~S}(\mathrm{O})\left(\mathrm{NCH}_{3}\right) \mathrm{CH}_{2} \mathrm{Li}$

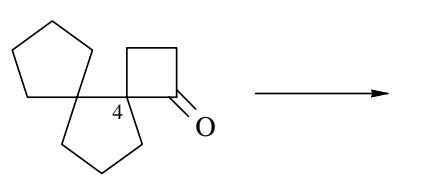

$\left(4 S^{*}\right)-51$

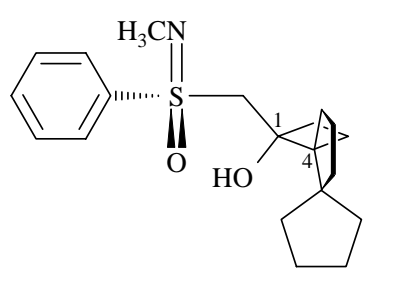

(SS,1R,4R)-59 (37\%, $>99 \%$ ee)

$\Delta \downarrow 87 \%$

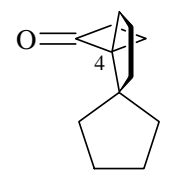

(4R)-51 (> 99\% еe)

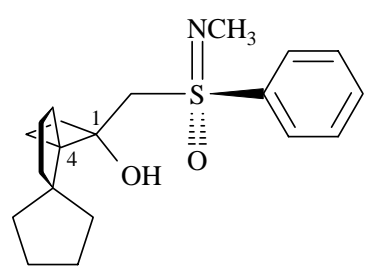

(SS,1S,4S)-61 (24\%, $>99 \%$ ee)

$\Delta \downarrow 96 \%$

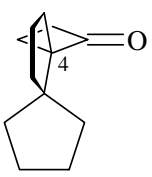

(4S)-51 (> 99\% ee)

Zur Freisetzung der enantiomerenreinen Ketone wurden beide $\beta$-Hydroxysulfoximine unter Argon auf $110^{\circ} \mathrm{C}$ erhitzt. Nach 14 Stunden waren beide Reaktionen beendet. Durch Säulenchromatographie erhielt man die reinen Ketone: $(4 R)-\mathbf{5 1}\left(>99 \%\right.$ ee, $[\alpha]_{\mathrm{D}}{ }^{20}=+145$, c 1.21, Aceton) in 87\% Ausbeute aus (SS,1R,4R)-59, und (4S)-51 (>99\% ee, $[\alpha]_{\mathrm{D}}{ }^{20}=-145$, c 1.14, Aceton) in 96\% Ausbeute aus (SS,1S,4S)-61 (Schema 39). Wie zu erwarten, zeigte (4R)51 einen starken positiven $\left(\theta_{307}=+6328, \mathrm{CH}_{3} \mathrm{OH}\right)$, und $(4 S)-51$ einen starken negativen Cotton-Effekt $\left(\theta_{307}=-6538, \mathrm{CH}_{3} \mathrm{OH}\right)$. Dies macht deutlich, dass die absolute Konfiguration von (4R)-51 und (4S)-51 auch über das Vorzeichen des Cotton-Effektes hätte bestimmt werden können.

\subsection{Racemattrennung von (4S*)-Dispiro[3.0.4.3]dodecan-1-on [(4S*)-51] mit (-)-Diisopinocampheylchloroboran}

Eine enantioselektive Reduktion mit (-)-Diisopinocampheylchloroboran [(-)-DIP-Cl] ${ }^{15}$ unter anschließender Rückoxidation mit Pyridiniumchlorochromat (PCC) ${ }^{16}$ hatten wir bereits früher erfolgreich zur Enanantiomerentrennung eines mit (4S*)-51 vergleichbaren Cyclobutanons eingesetzt. ${ }^{5}$ Bei (4S*)-51 war die Reduktion nach 18 h bei Raumtemperatur beendet. Aufarbeitung und Säulenchromatographie lieferten zwei diastereomere Alkohole in isolierten Ausbeuten von $21\left([\alpha]_{\mathrm{D}}{ }^{20}=+22.1\right.$, c 1.20 , Aceton) und $28 \%\left([\alpha]_{\mathrm{D}}{ }^{20}=+1.2\right.$, c 1.24 , Aceton), die durch Oxidation mit Pyridiniumchlorochromat in 61 bzw. 69\% Ausbeute in (4R)-51 bzw. (4S)-51 überführt werden konnten (Schema 40). (4R)-51 war mit einer spezifischen Drehung 
von $[\alpha]_{\mathrm{D}}^{20}=+145$ (c 1.21, Aceton) enantiomerenrein (>99\% ee), (4S)-51 mit einer spezifischen Drehung von $[\alpha]_{\mathrm{D}}{ }^{20}=-84.4$ (c 1.24, Aceton) dagegen nur angereichert (69\% ee).

\section{Schema 40}<smiles>O=C(O[C@H]1CC12CCC2)c1cc([N+](=O)[O-])cc([N+](=O)[O-])c1</smiles>

(1S,4R)- 104

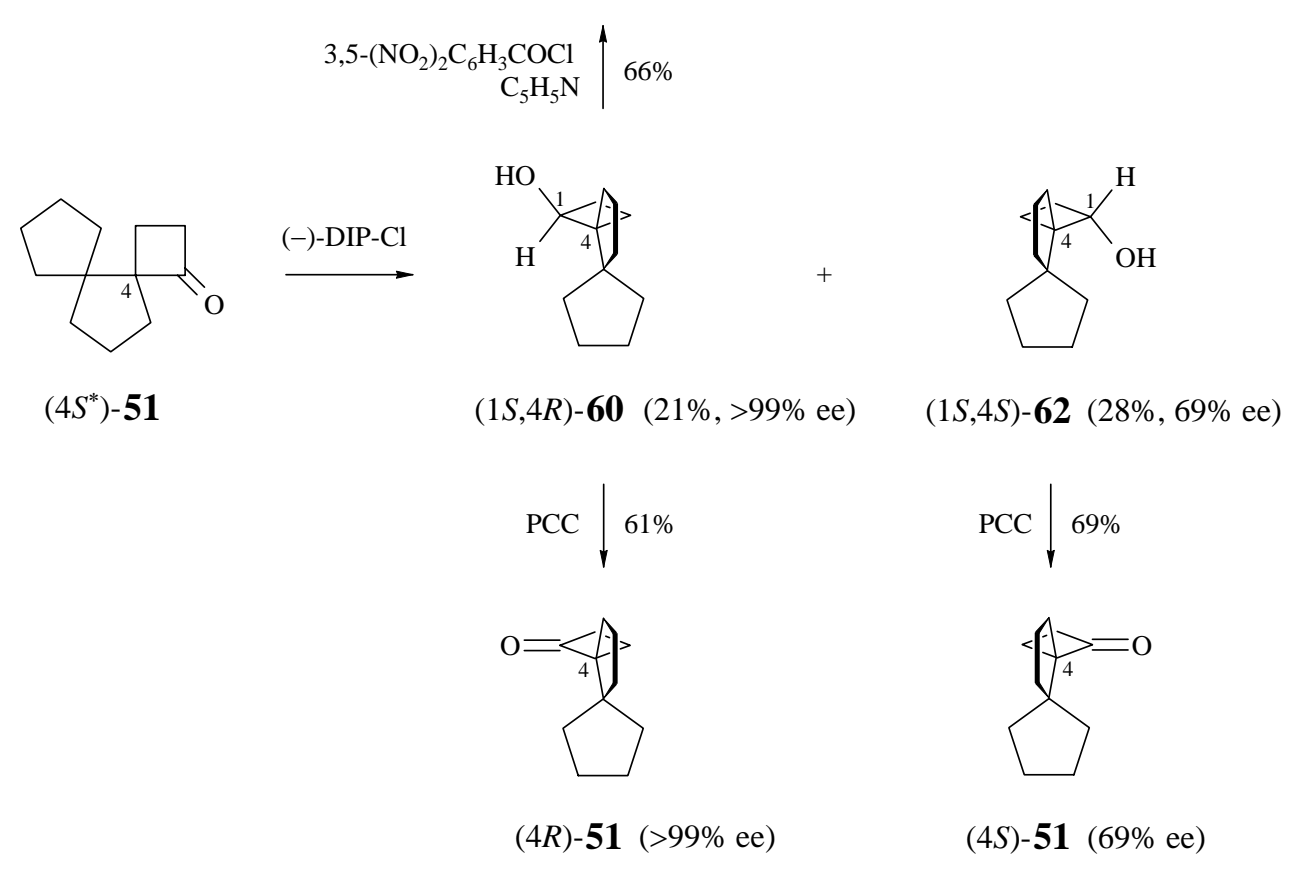

Zur Klärung der Stereochemie der Reduktion wurde der enantiomerenreine Minoritätsalkohol mit 3,5-Dinitrobenzoylchlorid umgesetzt und der gebildete Ester kristallographisch untersucht (vgl. 5, Abb. 6). Dabei stellte sich heraus, es sich bei dem Ester um $(1 S, 4 R)$ 104, und bei dem zugrunde liegenden Alkohol um $(1 S, 4 R)-60$ handelte. Damit stand gleichzeitig fest, dass der Majoritätsalkohol als (1S,4S)-62 zu formulieren war. Die Reduktionen von (4R)-51 und (4S)-51 waren demnach beide $S$-selektiv verlaufen. Dies steht mit früheren Beobachtungen ${ }^{15}$ im Einklang.

Zusammenfassend ist festzustellen, dass über eine Reduktion mit (-)-DIP-Cl und anschließende Rückoxidation lediglich (4R)-51 (Ausbeute 13\%) optisch rein erhalten werden kann. Der Weg über ß-Hydroxysulfoximine und deren Thermolyse liefert dagegen mit (4R)51 (Ausbeute 32\%) und (4S)-51 (Ausbeute 23\%) beide Enantiomere optisch rein. Für die enantioselektiven Synthesen von 8 und 15 wurde durchgängig (4R)-51 verwendet. 


\subsection{Synthese von (5R)-Trispiro[3.0.0.4.3.3]hexadecan [(5R)-15] und (P)-Trispiro- [4.0.0.4.3.3]heptadecan $[(P)-8]$}

Zur Darstellung der optisch reinen Trispirane (5R)-15 und (P)-8 haben wir die für die Racemate ausgearbeiteten Synthesen (vgl. 3.2.2 und 3.2.3) mit (4R)-51 wiederholt.

Zur Synthese von (5R)-15 wurde 1-Lithio-1-methylselenocyclobutan addiert, das in 40\% Ausbeute erhaltene 70:30-Gemisch aus (1R,4R)-58 und unumgesetztem (4R)-51 ohne weitere Reinigung oxidiert, und das nach Säulenchromatographie in 76\% Ausbeute isolierte (5R)-57 (> 99\% ee; $[\alpha]_{\mathrm{D}}{ }^{20}=-12.7$, c 1.23, Aceton) einer Wolff-Kishner-Reduktion unterworfen (Schema 41). Dies lieferte das gewünschte Trispiran (5R)-15 (>99\% ee; $[\alpha]_{\mathrm{D}}{ }^{20}=-30.7$, c 1.15, Chloroform) in einer Ausbeute von 66\%. (5R)-57 und (5R)-15 waren chemisch rein und ihre ${ }^{1} \mathrm{H}$ - und ${ }^{13} \mathrm{C}$-NMR-Daten mit denen der Racemate identisch.

\section{Schema 41}

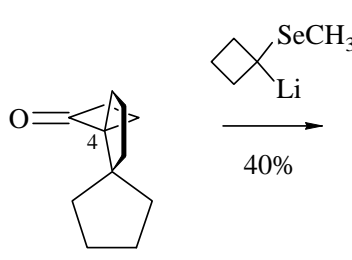

$(4 R)-51$

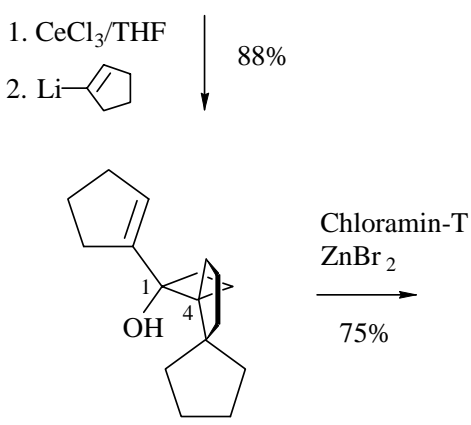

$(1 S, 4 R)-52$

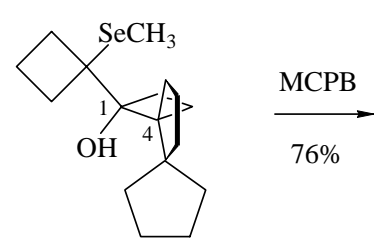

$(1 R, 4 R)-58$

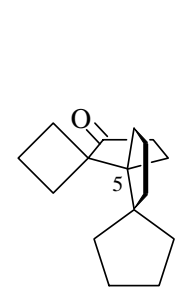

(5R)- 57
$\mathrm{H}_{2} \mathrm{NNH}_{2}$ $\mathrm{NaO}\left(\mathrm{CH}_{2}\right)_{2} \mathrm{O}\left(\mathrm{CH}_{2}\right)_{2} \mathrm{OH}$
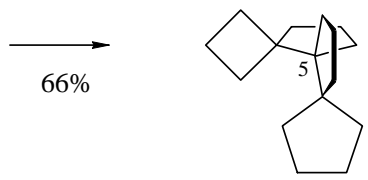

(5R)-15

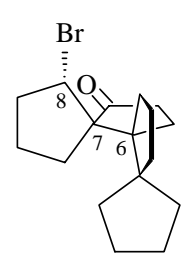

$(6 R, 7 R, 8 S)-54$

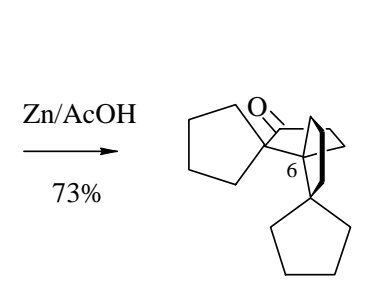

$(6 R)-55$
$\mathrm{H}_{2} \mathrm{NNH}_{2}$ $\mathrm{NaO}\left(\mathrm{CH}_{2}\right)_{2} \mathrm{O}\left(\mathrm{CH}_{2}\right)_{2} \mathrm{OH}$

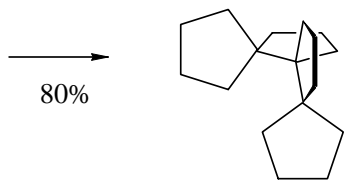

$(P)-8$

Zur Synthese von (P)-8 wurde Cyclopentenyllithium an (4R)-51 addiert, und das in 88\% Ausbeute isolierte Cyclopentenyl-cyclobutanol $(1 S, 4 R)-52\left(>99 \%\right.$ ee; $[\alpha]_{D}{ }^{20}=-46.1$, c 1.30, Aceton) mit Chloramin-T und Zinkbromid umgelagert. Das in 75\% Ausbeute isolierte Bromketon $(6 R, 7 S, 8 R)-54$ (> 99\% ee; $[\alpha]_{\mathrm{D}}{ }^{20}=-46.3$, c 1.24, Aceton) wurde reduktiv enthalogeniert, und das in 73\% Ausbeute erhaltene Keton (6R)-55 (>99\% ee; $[\alpha]_{\mathrm{D}}{ }^{20}=-127.0$, c 1.21, Aceton) nach Wolff-Kishner deoxigeniert (Schema 41). Dies lieferte $(P)-8$ (> 99\% ee; $[\alpha]_{\mathrm{D}}{ }^{20}=-62.6$, c 1.10 , Chloroform) in einer Ausbeute von $80 \%$. Auch hier waren die Produkte chemisch rein und ihre ${ }^{1} \mathrm{H}$ - und ${ }^{13} \mathrm{C}$-NMR-Daten mit denen der Racemate identisch. 
$5 \quad$ Kristallstrukturen von $\left(1 S, 5^{\prime} S, 11^{\prime} S\right)-(+)-79,\left(4 R^{*}, 5 S^{*}\right)-98,\left(6 S^{*}, 7 S^{*}, 8 R^{*}\right)-54$, $(\mathrm{S} S, 1 R, 4 R)-(+)-59,(\mathrm{SS}, 1 S, 4 S)-(+)-61$ und $(1 S, 4 R)-(+)-104$

Zur Bestimmung der relativen Konfigurationen von $\left(4 R^{*}, 5 S^{*}\right)-98,\left(6 S^{*}, 7 S^{*}, 8 R^{*}\right)-54$ und $(1 S, 4 R)-(+)-104$, sowie der absoluten Konfigurationen von (1S,5'S,11'S)-(+)-79, (SS,1R,4R)-(+)-59 und (SS,1S,4S)-(+)-61 wurden die genannten Verbindungen von $\mathrm{H}$. G. Schmidt, A. Pal und M. Noltemeyer vom Institut für Anorganische Chemie der Universität Göttingen kristallographisch untersucht.

Die Datensammlung erfolgte an einem Stoe-IPDS 2-Diffraktometer mit Mo- $\mathrm{K}_{\alpha}-$ Strahlung $\left[\left(1 S, 5^{\prime} S, 11^{\prime} S\right)-(+)-79,\left(4 R^{*}, 5 S^{*}\right)-98,\left(6 S^{*}, 7 S^{*}, 8 R^{*}\right)-54,(1 S, 4 R)-(+)-104\right]$ bzw. an einem Bruker-Smart 6000-Diffraktometer mit Cu-K $\mathrm{K}_{\alpha}$-Strahlung [(SS,1R,4R)-(+)-59, (SS,1S,4S)-(+)61]. Alle Strukturen wurden mit direkten Methoden bestimmt und anisotrop verfeinert (fullmatrix least-squares Verfeinerung gegen $\mathrm{F}^{2}$, SHELXTL). Die Wasserstoffatome wurden geometrisch positioniert. ORTEP-Darstellungen der Strukturen mit den kristallographischen Atomnumerierungen finden sich in den Abbildungen 1-6, die zugehörigen Kristalldaten sowie Einzelheiten über die Strukturverfeinerung in Tabelle 1.

Die Struktur von (1S,5'S,11'S)-(+)-79 (Abb. 1) bestätigte die $S$-selektive Reduktion

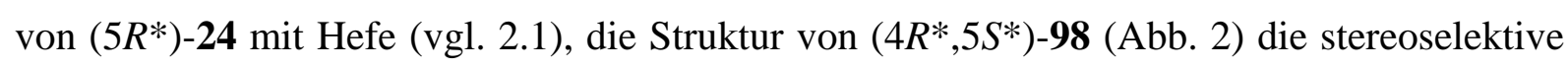
Umlagerung von $\left(1 S^{*}, 5 S^{*}\right)-42$ zu einem „endo“-Keton (vgl. 3.1.1), die Struktur von $\left(6 S^{*}, 7 S^{*}, 8 R^{*}\right)-54$ (Abb. 3) die regio- und stereoselektive Umlagerung von $\left(1 R^{*}, 4 S^{*}\right)-52 \mathrm{zu}$ einem $\beta$-Bromketon (vgl 3.2.2), und die Struktur von (1S,4R)-(+)-104 (Abb. 6) die $S$-selektive Reduktion von (4S*)-51 mit (-)-DIP-Cl (vgl 4.2). Die Strukturen von (SS,1R,4R)-(+)-59 (Abb. 4) und (SS,1S,4S)-(+)-61 (Abb. 5) erlaubten die Festlegung der absoluten Konfiguration der Enantiomeren von (4S*)-51 nach Racemattrennung (vgl. 4.1). 


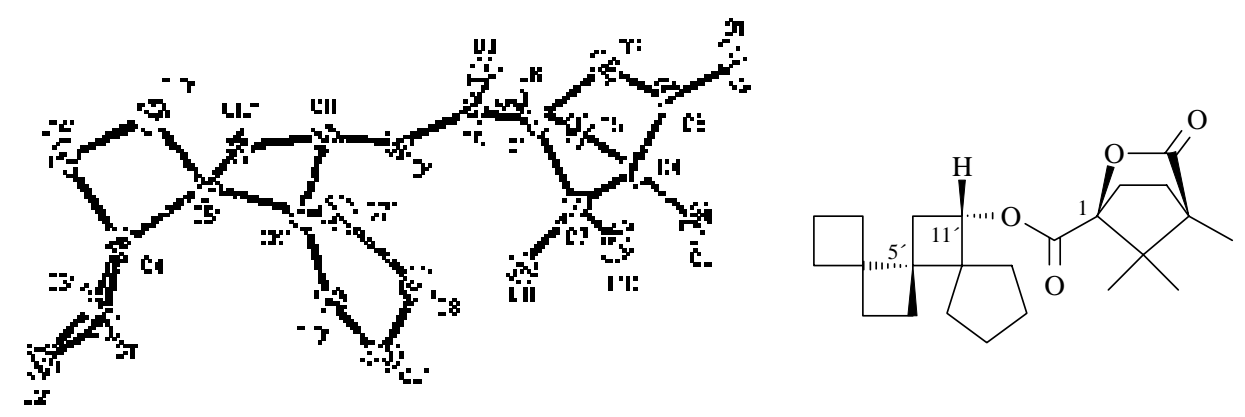

Abb. 1 Kristallstruktur von (1S,5'S,11'S)-(+)-79 ohne Wasserstoffatome (H. G. Schmidt, M. Noltemeyer)
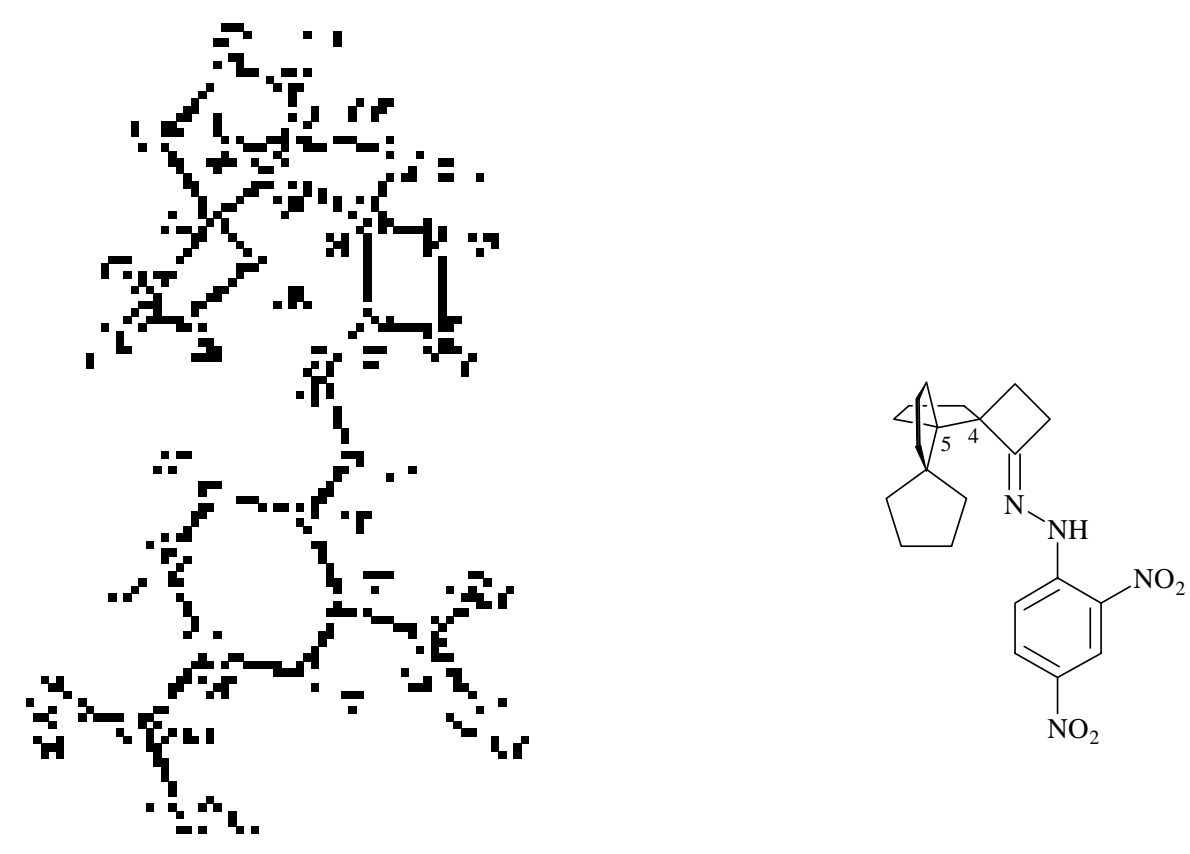

Abb. 2 Kristallstruktur von $\left(4 R^{*}, 5 S^{*}\right)-\mathbf{9 8}$ ohne Wasserstoffatome (H. G. Schmidt, M. Noltemeyer)
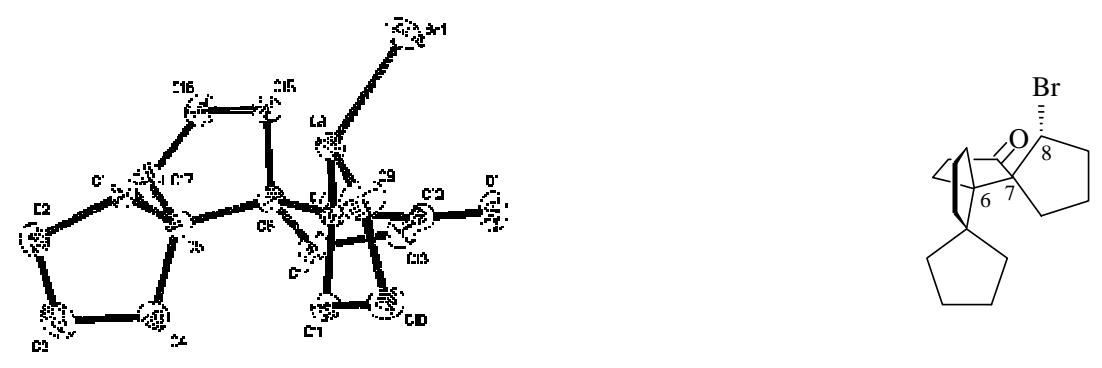

Abb. 3 Kristallstruktur von $\left(6 S^{*}, 7 S^{*}, 8 R^{*}\right)-54$ ohne Wasserstoffatome (H. G. Schmidt, M. Noltemeyer) 

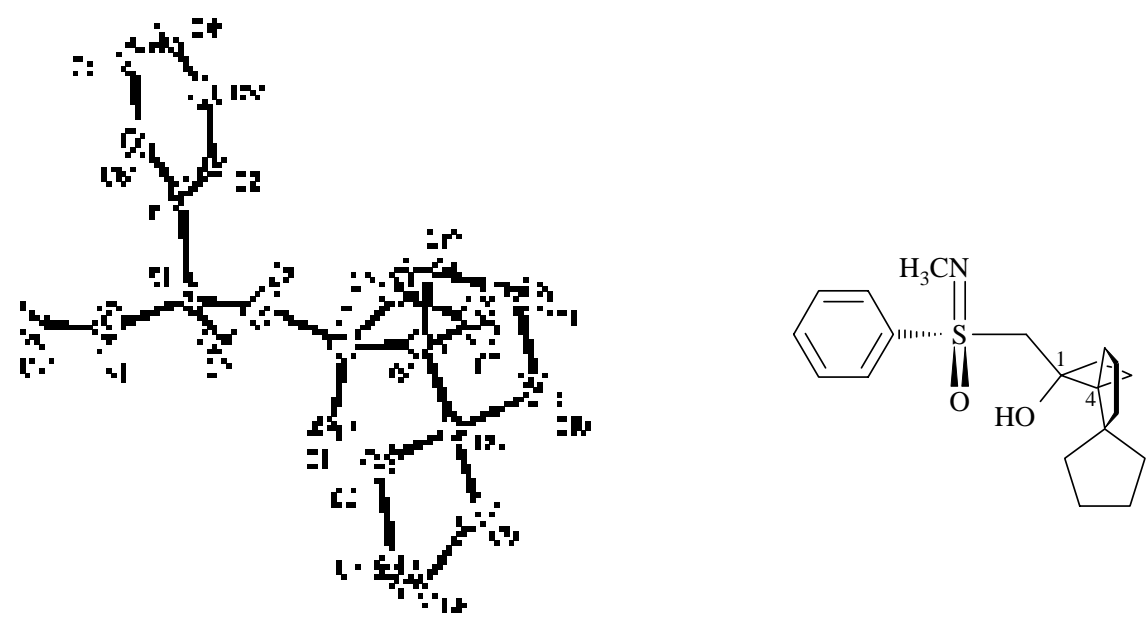

Abb. 4 Kristallstruktur von (SS,1R,4R)-(+)-59 ohne Wasserstoffatome (A. Pal, M. Noltemeyer)
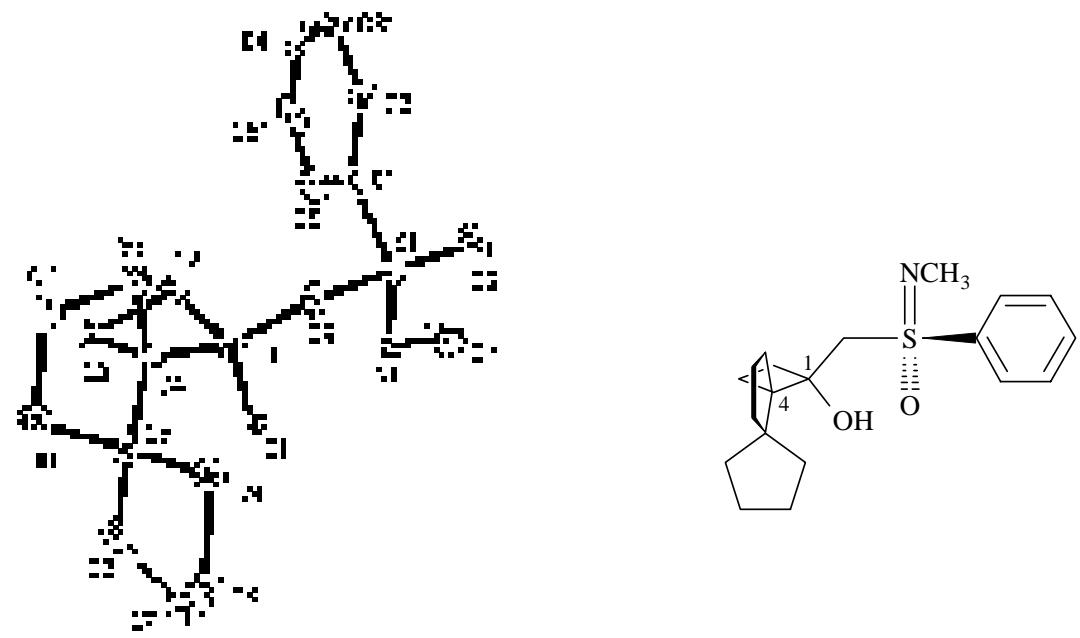

Abb. 5 Kristallstruktur von (SS,1S,4S)-(+)-61 ohne Wasserstoffatome (A. Pal, M. Noltemeyer)
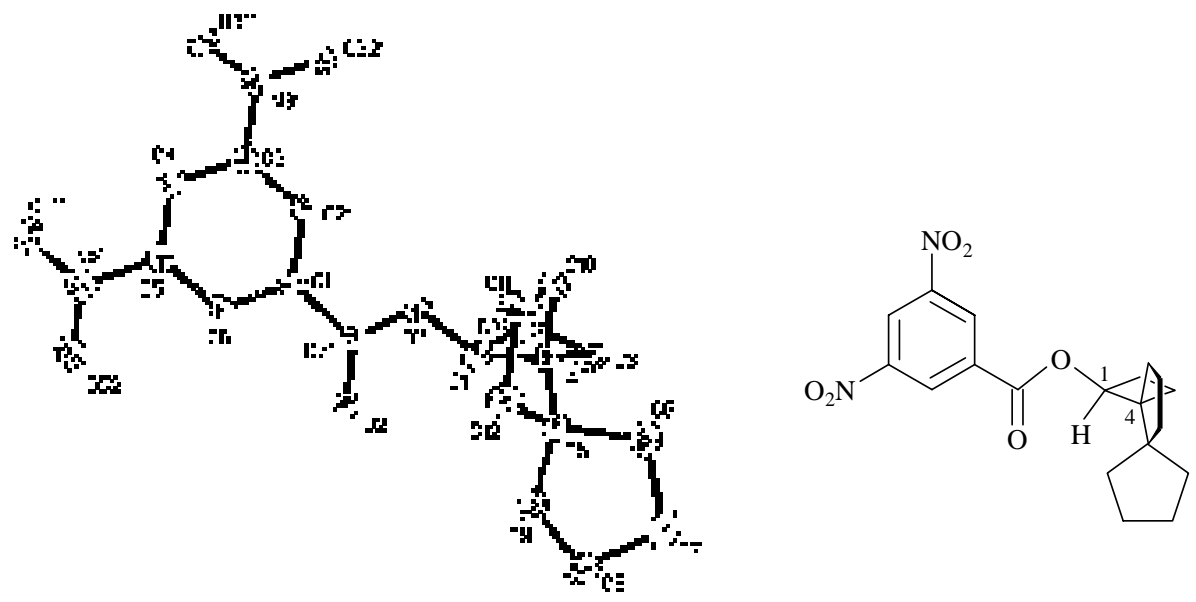

Abb. 6 Kristallstruktur von (1S,4R)-(+)-104 ohne Wasserstoffatome (A. Pal, M. Noltemeyer) 


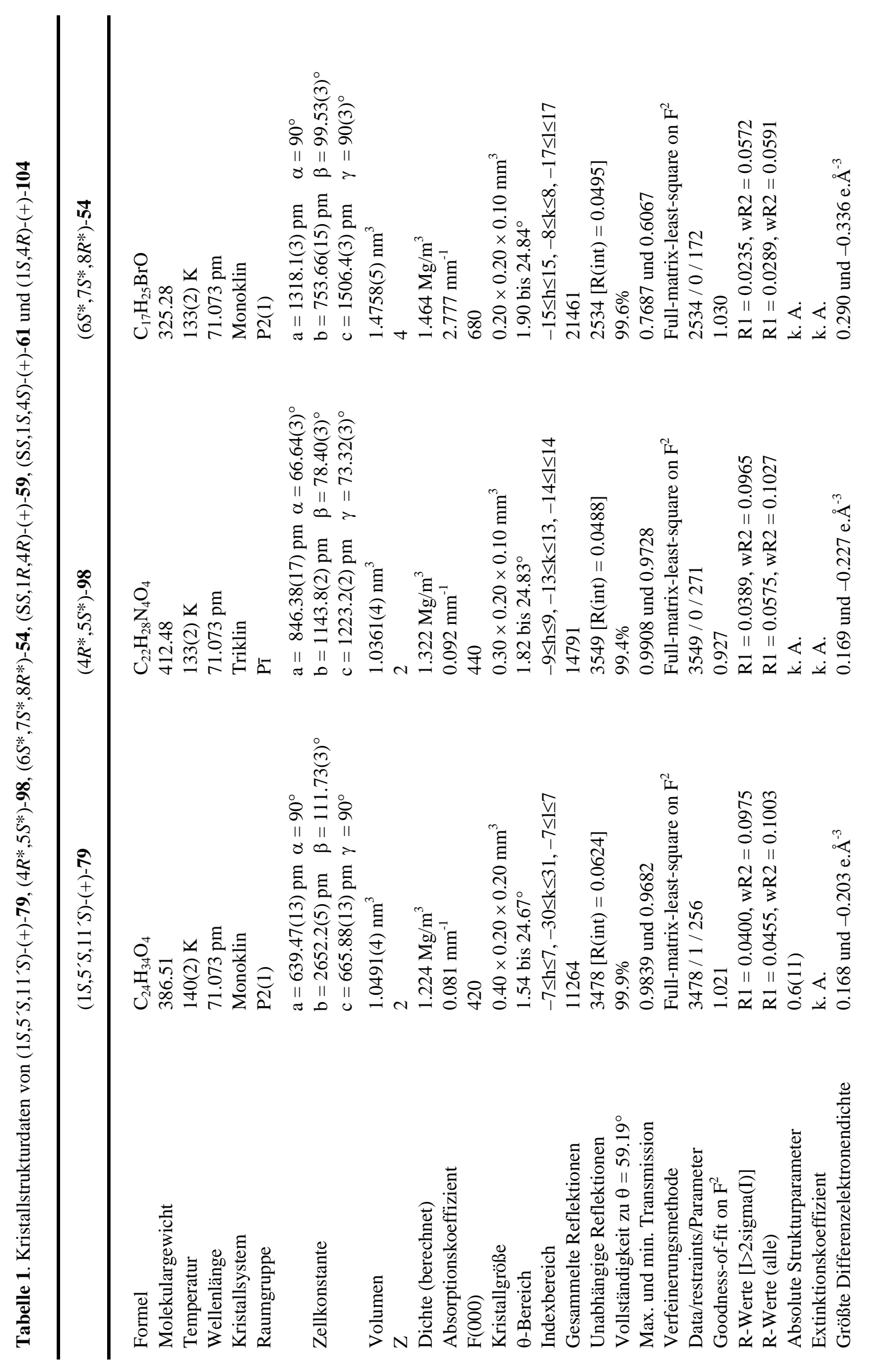




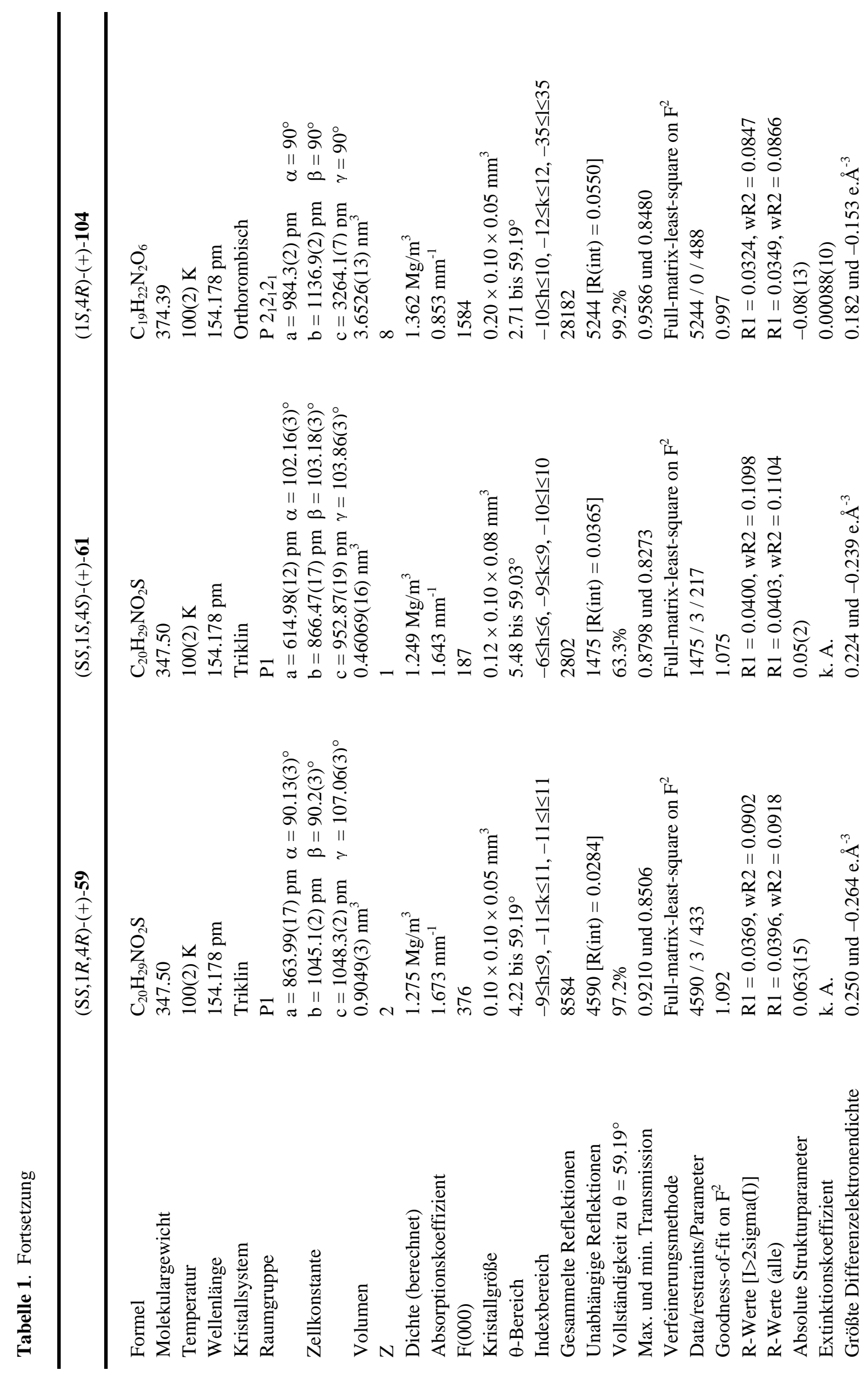




\section{Diskussion und Ausblick}

Als Grundlage für die Diskussion sowie für spätere ab initio-Rechnungen haben wir die spezifischen Drehungen der pseudohelicalen Trispirane (5R)-13, (5S)-14 und (5R)-15, sowie des helicalen Trispirans $(P)-\mathbf{8}$ bei verschiedenen Wellenlängen gemessen und den Werten der helicalen Trispirane (P)-6 und $(P)-7$ [abgeleitet von $(M)-\mathbf{6}^{1,2}$ bzw. $(M)-7^{4}$ ] gegenübergestellt (Tabelle 2).<smiles>C1CC1C1(C2CC2)CC1</smiles>

$(P)-6$

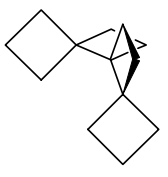

$(P)-7$

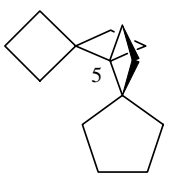

(5R)-13

(pseudo-P)

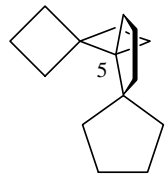

(5S)-14 (pseudo-P)
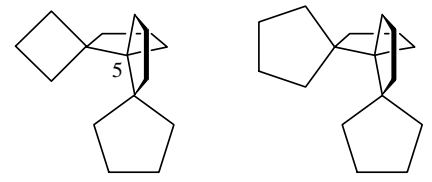

(5R)-15 (pseudo-P)
$(P)-8$

\begin{tabular}{ccccccc}
\hline & $\begin{array}{c}\text { c } 1.2 \\
{[\alpha]_{\mathrm{nm}}{ }^{20}}\end{array}$ & $\begin{array}{l}\text { c } 1.08 \\
{[\alpha]_{\mathrm{nm}}{ }^{20}}\end{array}$ & $\begin{array}{l}\text { c } 1.19 \\
{[\alpha]_{\mathrm{nm}}{ }^{20}}\end{array}$ & $\begin{array}{l}\text { c } 1.22 \\
{[\alpha]_{\mathrm{nm}}{ }^{20}}\end{array}$ & $\begin{array}{l}\text { c } 1.15 \\
{[\alpha]_{\mathrm{nm}}{ }^{20}}\end{array}$ & $\begin{array}{l}\text { c } 1.10 \\
{[\alpha]_{\mathrm{nm}}{ }^{20}}\end{array}$ \\
\hline 589 & +193.7 & +63.3 & -4.1 & -0.9 & -30.7 & -62.6 \\
578 & - & +65.9 & -4.5 & -0.9 & -31.9 & -65.2 \\
546 & +229.7 & +74.9 & -4.7 & -1.1 & -36.1 & -74.0 \\
436 & +400.2 & +126.9 & -6.2 & -1.2 & -60.0 & -124.3 \\
365 & +648.2 & +199.1 & -6.3 & -0.7 & -92.8 & -192.6 \\
\hline
\end{tabular}

Tabelle 2. Spezifische Drehungen von $(P)-\mathbf{6},(P)-\mathbf{7},(5 R)-\mathbf{1 3},(5 S)-\mathbf{1 4},(5 R)-15$ und $(P)-\mathbf{8}$ bei verschiedenen Wellenlängen in Chloroform.

Bei einem Vergleich fällt auf, dass die spezifische Drehung beim Übergang von $(P)-\mathbf{6}$ $\left([\alpha]_{20}^{\mathrm{D}_{20}}=+193\right) \mathrm{zu}(P)-7\left([\alpha]^{\mathrm{D}_{20}}=+63\right)$ stark fällt, und dass dieser Trend beim Übergang zu den pseudo-helicalen Trispiranen (5R)-13 $\left([\alpha]_{20}^{\mathrm{D}_{20}}=-4\right)$ und $(5 S)-14\left([\alpha]_{20}^{\mathrm{D}_{20}}=-1\right)$ anhält. Noch interessanter ist der Befund, dass die spezifischen Drehungen des pseudo-helicalen Trispirans (5R)-15 $\left([\alpha]_{20}^{\mathrm{D}_{20}}=-31\right)$ und des helicalen Trispirans $(P)-\mathbf{8}\left([\alpha]^{\mathrm{D}}{ }_{20}=-63\right)$ nicht etwa bei Null, sondern, mit stark steigenden Beträgen, deutlich im Negativen liegen. Dies zeigt, dass in der Reihe 6-7-13-14-15-8 der von der jeweiligen inneren Helix geleistete positive Beitrag zum Drehwert durch negative Beiträge der Vier- und Fünfringe zunächst kompensiert, und dann überkompensiert wird. Dies liegt mit hoher Wahrscheinlichkeit in der Geometrie der jeweiligen Konformationen begründet. 
Um diesen Punkt näher zu untersuchen, wurden zusätzlich zu früheren Kraftfeldrechnungen an $(M)-\mathbf{7},{ }^{4}$ auch solche an (5R)-13, (5S)-14, (5R)-15 und $(P)-\mathbf{8}$ durchgeführt. Dabei verwendeten wir unsere Suchroutine HUNTER ${ }^{69}$ in Verbindung mit MM3 ${ }^{70}$ und ermittelten alle innerhalb $3 \mathrm{kcal} / \mathrm{mol}$ oberhalb des absoluten Minimums liegenden Konformationen. Ihre Bildungsenthalpien sind, zusammen mit denen von (M)-7, in Abb.7 zusammengestellt. Dort finden sich ferner Abbildungen der absoluten Minima [bei $(M)-7$ gespiegelt], sowie Angaben über die Diederwinkel der inneren Helix, und die Diederwinkel derjenigen asymmetrischen C4-Einheiten, in denen eine Bindung der inneren Helix von Bindungen ein- und desselben Ringes flankiert wird. Blickrichtung ist in allen Fällen längs der helicalen Achse.

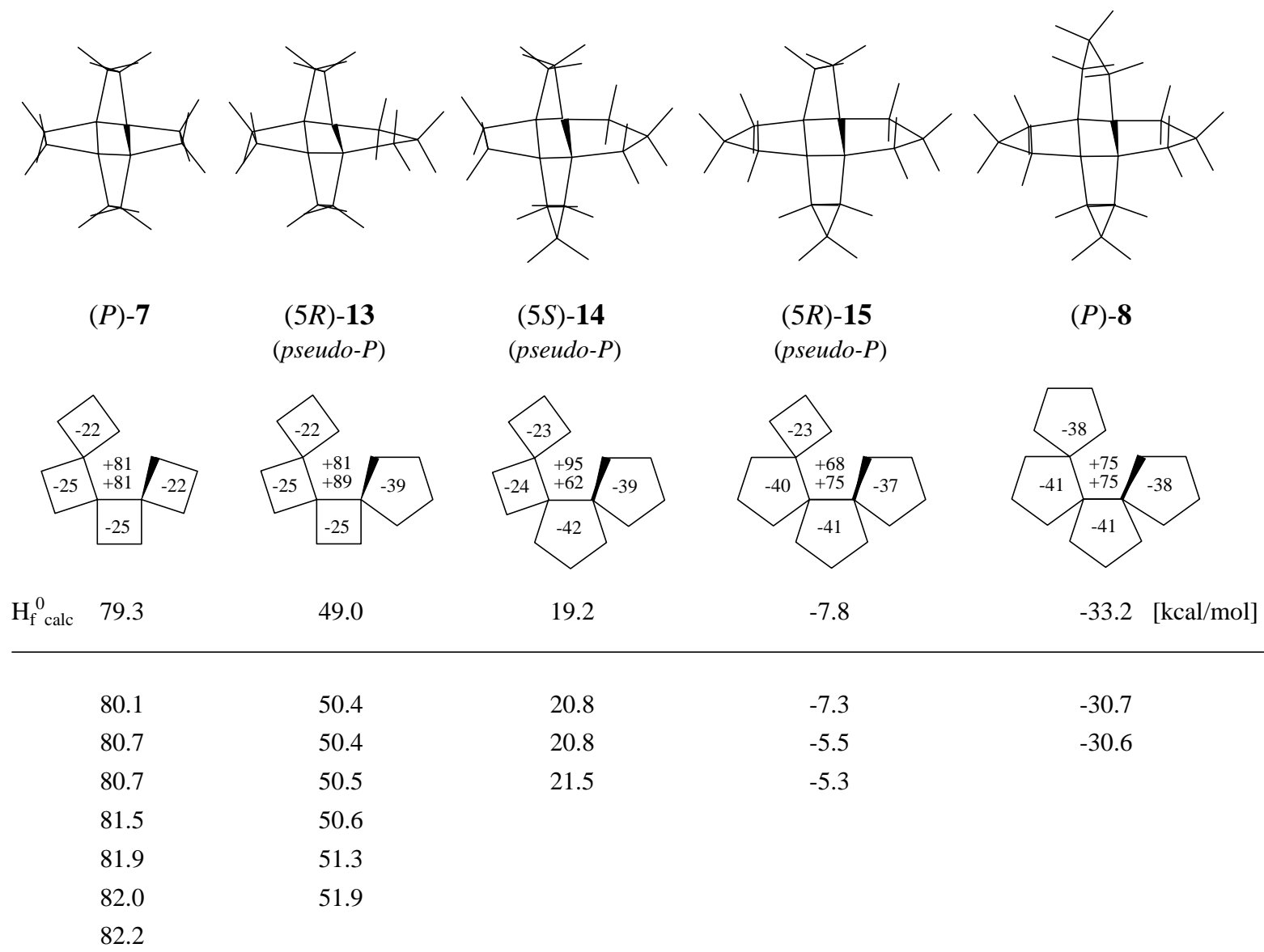

Abb.7 Bildungsenthalpien (in kcal/mol) aller Konformationen von (P)-7, (5R)-13, (5S)-14, $(5 R)-15$ und $(P)-8$ bis 3 kcal über dem absoluten Minimum. Die absoluten Minima sind mit Blickrichtung längs der helicalen Achse dargestellt und ausgewählte Diederwinkel (vgl. Text) angegeben. Alle Rechnungen erfolgten mit HUNTER ${ }^{69}$ in Verbindung mit $\mathrm{MM}^{70}$.

Wie Abb.7 zu entnehmen ist, sinkt die Zahl der Konformationen bis 3 kcal über dem absoluten Minimum von acht in (P)-7 über sieben in (5R)-13 und jeweils vier in (5S)-14 und (5R)-15 auf drei in $(P)-8$. In $(P)-8$ ist das absolute Minimum gegenüber dem nächstliegenden 
Konformer mit $2.5 \mathrm{kcal}$ am stärksten begünstigt. Es könnte daher sein, dass die spezifische Drehung von $(P)$-8 von einem einzigen Konformer bestimmt wird.

Beschränkt man sich auf einen Vergleich der die optischen Eigenschaften am stärksten prägenden absoluten Minima, so fällt auf, dass sie sehr ähnliche Geometrien besitzen. Die beiden Diederwinkel der inneren Helix sind, wie für pseudo-P- und $P$-Helices zu fordern, positiv, die Diederwinkel derjenigen Bindungen eines jeden Ringes, die eine Bindung der inneren Helix einschließen, dagegen durchweg negativ. Letztere liegen für Cyclobutane um $25^{\circ}$, und für Cyclopentane um $-40^{\circ}$. Es scheint demnach durchaus einsichtig, dass der positive Beitrag, den die jeweilige innere Helix zum Drehwert liefert, von Cyclobutanen langsamer, von Cyclopentanen dagegen schneller aufgezehrt wird.

Natürlich ist uns bewusst, dass die gemessenen spezifischen Drehungen Boltzmanngewichtete Mittel der Drehwerte real populierter Konformationen darstellen. Eine genauere Interpretation wird daher erst dann möglich sein, wenn ab initio-Rechnungen an einer genügend hohen Zahl niederenergetischer Konformationen vorliegen. Dazu müssen zunächst die relevanten Konformationen ermittelt, und anschließend ihre spezifischen Drehungen berechnet und gewichtet werden. Derartige Rechnungen wurden im Arbeitskreis von Herrn Prof. Grimme, Institut für theoretische Chemie der Universität Münster, für $(M)-7$ bereits durchgeführt, ${ }^{9}$ erforderten allerdings wegen der hohen Zahl an Boltzmann-relevanten Konformationen einen erheblichen Aufwand. Von den in dieser Arbeit dargestellten Trispiranen (5R)-13, (5S)14, (5R)-15 und $(P)-8$ scheint $(P)-8$ wegen der zu erwartenden geringen Zahl an Boltzmannrelevanten Konformationen (vgl. Abb.7) für weiterführende Rechnungen am ehesten geeignet. Sobald derartige Rechnungen durchgeführt sind, wird ein genauerer Vergleich der optischen Eigenschaften von $(P)-6$, $(P)-7$ und $(P)-8$, d.h. von Helices aus spiroanellierten drei-, vierund fünfgliedrigen Ringen möglich sein. 


\section{ZUSAMMENFASSUNG}

Aufgabe der vorliegenden Arbeit war es, die pseudohelicalen Trispirane 13, 14 und 15, sowie das helicale Trispiran 8 racemisch und optisch rein zu synthetisieren, ihre spezifischen Drehungen bei verschiedenen Wellenlängen zu bestimmen, und mit den Drehungen der bekannten helicalen Trispirane $\mathbf{6}^{1,2}$ und $\mathbf{7}^{4}$ vergleichend zu diskutieren (Schema 44). Ziel war es, den Einfluss statischer (Identitätsperiode, Ganghöhe und Länge der Helix) und dynamischer Parameter (konformationelle Gleichgewichte) auf die optischen Eigenschaften helicaler Trispirane einzugrenzen und für die dazu notwendigen ab initio-Rechnungen experimentelle Daten zur Verfügung zu stellen.

\section{Schema 44}

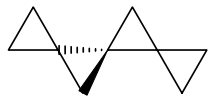

$(M)-6$

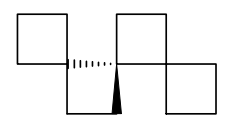

$(M)-7$

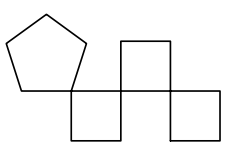

13

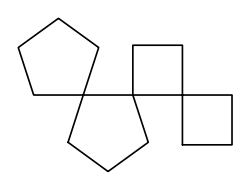

14

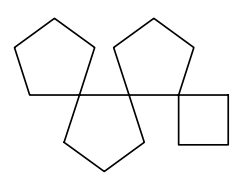

15

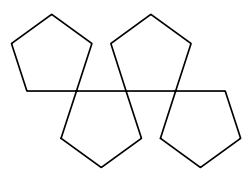

8

$$
[\alpha]_{D}^{20}=-193.7 \quad[\alpha]_{D}^{20}=-63.3
$$

Von den angestrebten Synthesen wurden die von 13 und 14 über eine [2+2]Cycloaddition des Keteniminiumsalzes 64 an 1-Methylenspiro[3.3]heptan (21) realisiert. Direkte Deoxigenierung des gebildeten Trispiroketons 24 lieferte 13, vorherige Ringerweiterung mit Diazomethan führte zu 14 (Schema 45). Die zur Darstellung optisch reiner Vertreter notwendige Racemattrennung gelang durch dia- und enantioselektive Reduktion von 24 mit Hefe. Das enantiomerenrein zurückbleibende (5R)-24 wurde als Edukt für Synthesen von (5R)-13 und (5S)-14 genutzt.

\section{Schema 45}

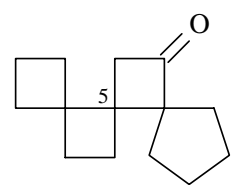

rac-24
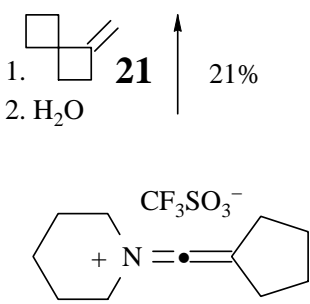

64
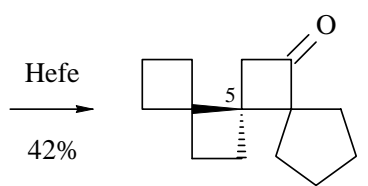

$(5 R)-24$

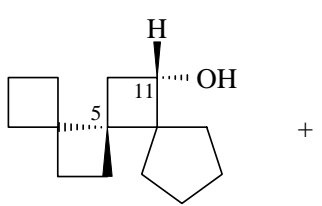

(5S,11S)- $\mathbf{2 8}$

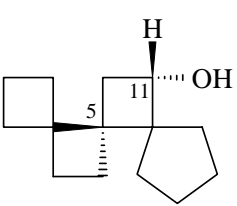

$(5 R, 11 S)-29$

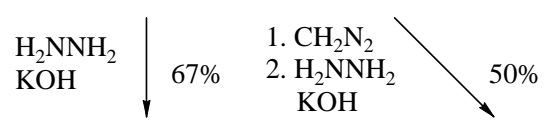

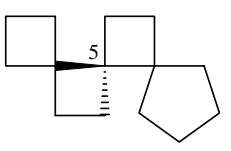

$(5 R)-13$

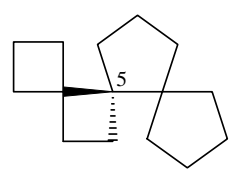

(5S)-14 
Weitere [2+2]Cycloadditionen zu potentiellen Vorläufern von 15 und 8 gelangen nicht (vgl. 1.2). Daraufhin wurden die Zahl der zu anellierenden Ringe auf einen beschränkt und mit 30 und 51 zwei fertige Dispirane in Anellierungsreaktionen eingesetzt. Ausgehend von Cyclobutyliden-cyclopentan (69) gelang ihre Synthese über Oxaspiroalkan-CycloalkanonUmlagerungen (69-71-51) bzw. durch Ringerweiterung mit 4-Nitrobenzolsulfonsäureazid, Cycloalkylierung und Keton-Keton-Umlagerung (69-32-31-30) (Schema 46).

\section{Schema 46}<smiles>O=C1CCCC1</smiles>

66

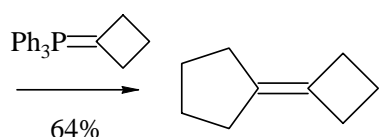

69

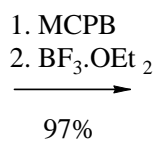

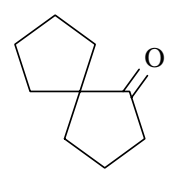

71

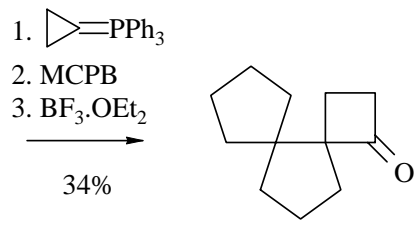

51

\begin{tabular}{l|l} 
1. $4-\mathrm{NO}_{2} \mathrm{C}_{6} \mathrm{H}_{4} \mathrm{SO}_{2} \mathrm{~N}_{3}$ & \. $\mathrm{HCl} \%$
\end{tabular}<smiles>O=C1CCCCC12CCC2</smiles>

32

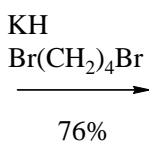

$6 \%$

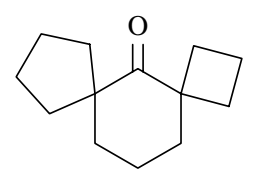

31

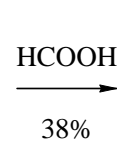

$38 \%$

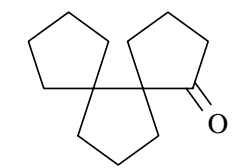

30

Die Anellierung eines Cyclobutanons an $\mathbf{3 0}$ gelang durch Addition von 1-Ethoxyvinyllithium, Cyclopropanierung und Umlagerung, führte jedoch zu einem Trispiroketon mit endo-ständiger Carbonylgruppe und damit geringer Reaktivität (30-41-42-43) (Schema 47).

\section{Schema 47}
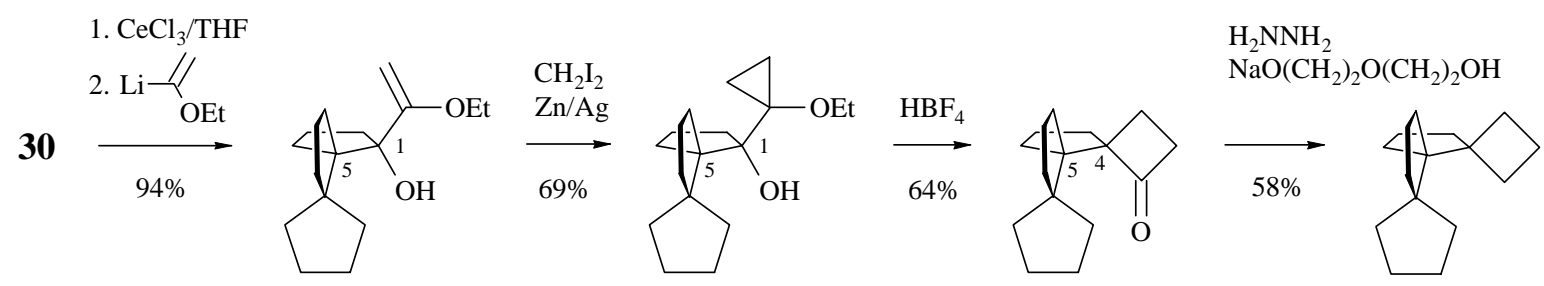

$\left(1 S^{*}, 5 S^{*}\right)-\mathbf{4 1}$

$\left(1 S^{*}, 5 S^{*}\right)-42$

$\left(4 R^{*}, 5 S^{*}\right)-43$

15

$$
\mathrm{Ph}_{3} \mathrm{P}=\mathrm{CH}_{2} \sqrt{87 \%}
$$
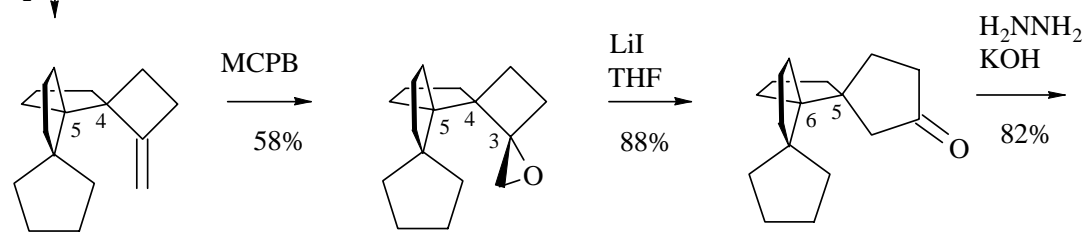

$\left(4 R^{*}, 5 S^{*}\right)-44$

$\left(3 S^{*}, 4 R^{*}, 5 S^{*}\right)-46$

(5S*,6S*)- 35

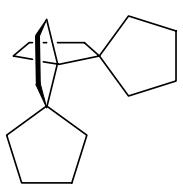


Seine Deoxigenierung zu 15 erwies sich als schwierig, und eine Ringerweiterung mit Diazomethan gelang nicht. Eine ersatzweise vorgenommene Methylenierung, Epoxidierung, und Oxaspirohexan-Cyclopentanon-Umlagerung (43-44-46-35) verlängerte die Synthese von 8 zusammen mit der abschließenden Deoxigenierung auf sieben Schritte. Wir haben deshalb mit 51 kürzere Wege zu realisieren versucht.

Tatsächlich lieferte eine Addition von 1-Lithio-cyclopenten, gefolgt von einer Umlagerung mit positiviertem Brom, einer reduktiven Enthalogenierung und einer Deoxigenierung 8 in lediglich vier Schritten (51-52-54-55-8). Der Versuch einer analogen Synthese von 15 scheiterte im Enthalogenierungsschritt (vgl. 3.2.2). 15 konnte jedoch in einer auf drei Schritte verkürzten Synthese über eine Addition von 1-Lithio-1-methylselenocyclobutan und eine Ringerweiterung des resultierenden $\beta$-Hydroxyselenids 58 erhalten werden (51-58-57-15) (Schema 48).

\section{Schema 48}

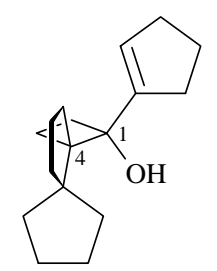

$\left(1 R^{*}, 4 S^{*}\right)-52$

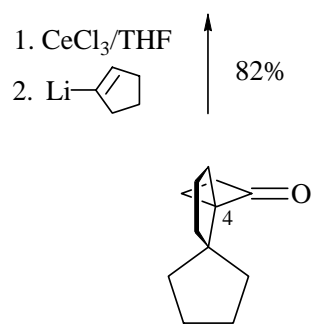

$\left(4 S^{*}\right)-51$

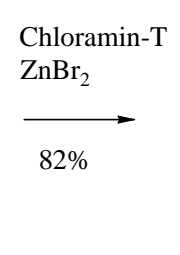

$\left(6 S^{*}, 7 S^{*}, 8 R^{*}\right)-54$

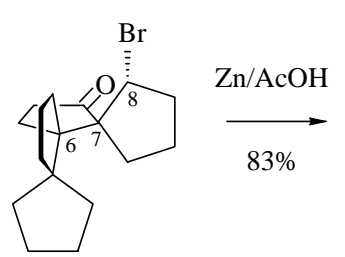

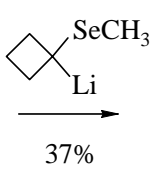

$\left(1 S^{*}, 4 S^{*}\right)-\mathbf{5 8}$

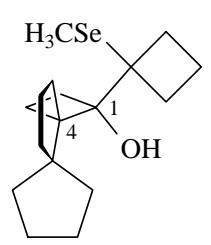

$\underset{70 \%}{\stackrel{\mathrm{MCPB}}{\longrightarrow}}$

$70 \%$

$\left(6 S^{*}\right)-55$

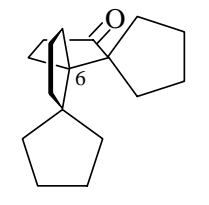

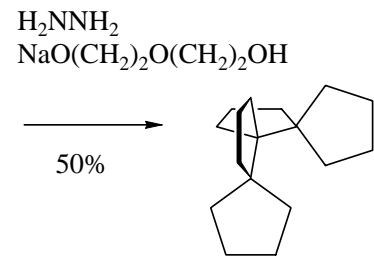

8

$\mathrm{H}_{2} \mathrm{NNH}_{2}$ $\mathrm{NaO}\left(\mathrm{CH}_{2}\right)_{2} \mathrm{O}\left(\mathrm{CH}_{2}\right)_{2} \mathrm{OH}$

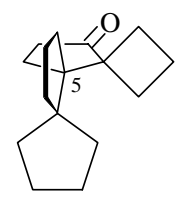

$\left(5 S^{*}\right)-57$

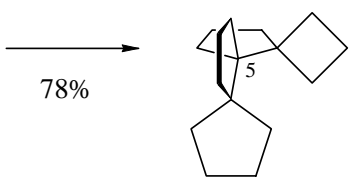

$\left(5 S^{*}\right)-15$

Die Racemattrennung von 51 gelang (a) durch Umsetzung mit (+)-(S)-N,S-Dimethyl$S$-phenylsulfoximin, und (b) durch Reduktion mit (-)-Diisopinocampheylchloroboran [(-)DIP-Cl] (Schema 49). Im ersten Fall bildeten sich zwei diastereomere Sulfoximine 59 und 61, die nach säulenchromatographischer Trennung zunächst kristallographisch charakterisiert und dann durch thermische Fragmentierung in die enantiomerenreinen Ketone (4R)-51 und (4S)51 überführt wurden. Im zweiten Fall resultierten zwei diastereomere Alkohole $\mathbf{6 0}$ und $\mathbf{6 2}$, von denen sich nach Rückoxidation 60 als enantiomerenrein, 62 dagegen als optisch nur angereichert erwies. 


\section{Schema 49}

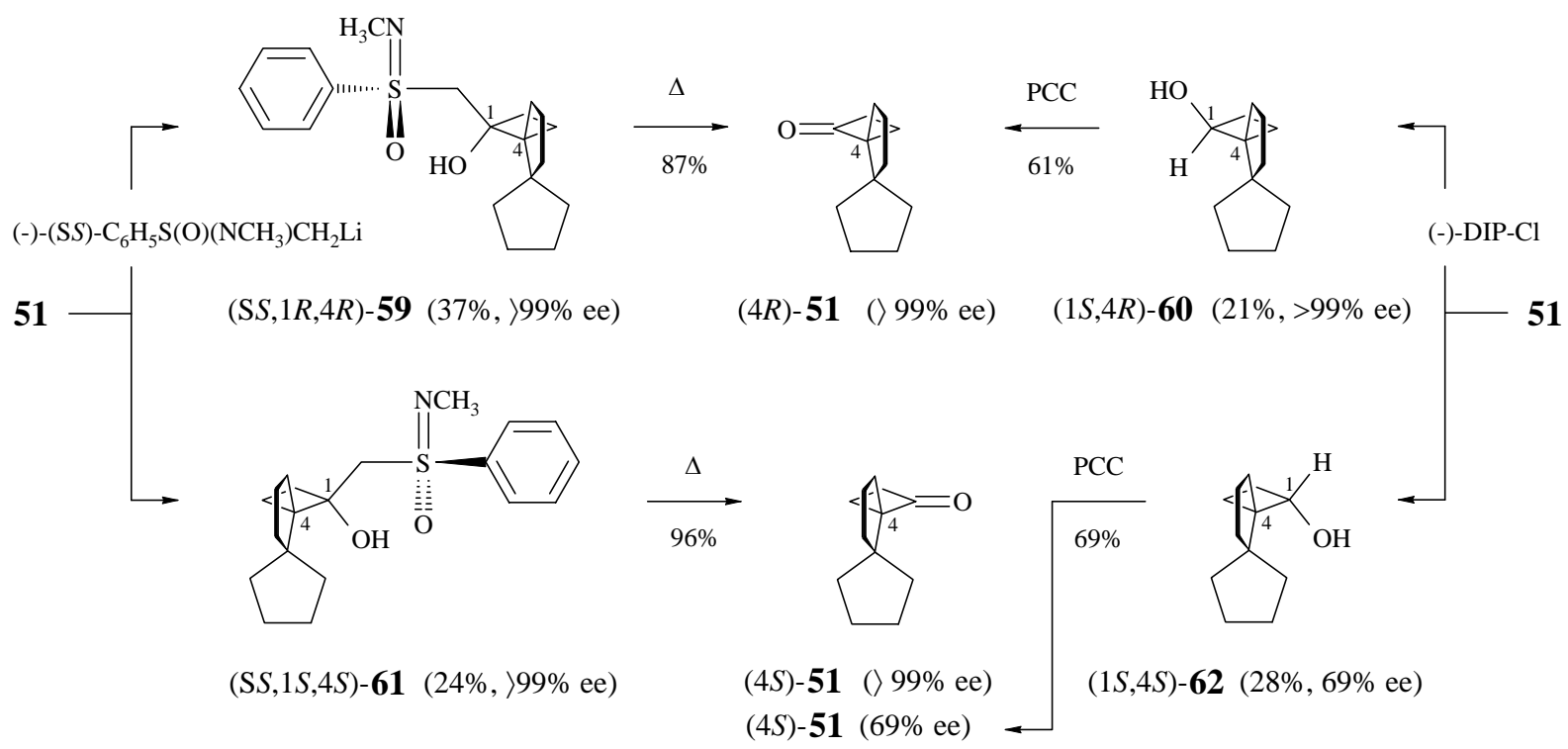

Für die asymmetrischen Synthesen wurde durchgängig (4R)-51 verwendet. Sie führten zu (5R)-15 und $(P)-8$. Ihre spezifischen Drehungen sind mit denen von $(P)-6$ [abgeleitet von $(M)-6],{ }^{1,2}(P)-7$ [abgeleitet von $\left.(M)-7\right]^{4}(5 R)-13$, und (5S)-14 in Schema 50 aufgeführt. Aus einem Vergleich geht hervor, dass beim Übergang von dem geometrisch fixierten Trispiran $(P)$-6 zu den konformativ beweglichen Trispiranen $(P)-7,(5 R)-13,(5 S)-14,(5 R)-15$ und $(P)-\mathbf{8}$ der positive Beitrag, den die innere Helix zum Drehwert leistet, durch zunehmend negative Beiträge der spiroanellierten Ringe zunächst kompensiert, und dann überkompensiert wird. Eine genauere Interpretation wird jedoch erst dann möglich sein, wenn ab initio-Rechnungen zu (5R)-13, (5S)-14, (5R)-15 und $(P)-8$ vorliegen.

\section{Schema 50}<smiles>CC1(C2CC2)CC1</smiles>

(P)-6

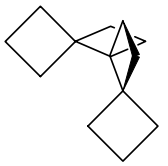

(P)-7

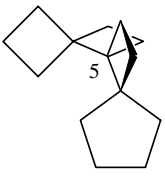

(5R)-13

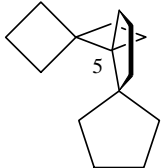

(5S)-14 (pseudo-P)
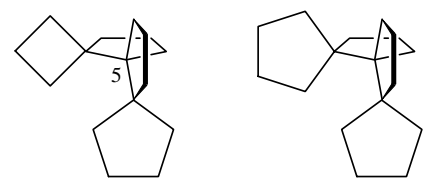

(5R)-15 (pseudo-P)
(P)-8

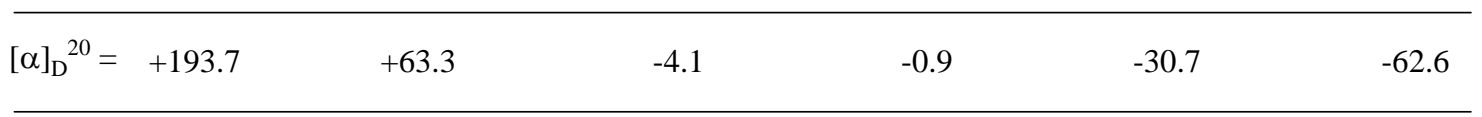




\section{EXPERIMENTELLER TEIL}

\section{Allgemeine Angaben}

IR-Spektren wurden mit einem Spektrometer Modell 298 der Firma Perkin Elmer aufgenommen. ${ }^{1}$ H- und ${ }^{13}$ C-NMR-Spektren wurden auf einem Gerät AMX 300 der Firma Bruker bzw. einem Gerät VXR 500 oder VXR 600 der Firma Varian aufgenommen. Chemische Verschiebungen sind als $\delta$-Werte in ppm angegeben und beziehen sich auf Tetramethylsilan ( $\delta=0.00)$ als internen Standard. Bei Verwendung anderer Standards wurden folgende chemische Verschiebungen zugrunde gelegt: $\delta_{\mathrm{H}}\left(\mathrm{CHCl}_{3}\right)=7.24, \delta_{\mathrm{H}}\left(\mathrm{C}_{6} \mathrm{D}_{5} \mathrm{H}\right)=7.15, \delta_{\mathrm{H}}$ $\left(\mathrm{C}_{2} \mathrm{HDCl}_{4}\right)=5.99, \delta_{\mathrm{H}}\left(\mathrm{CDCl}_{2} \mathrm{H}\right)=5.33, \delta_{\mathrm{D}}\left(\mathrm{CDCl}_{3}\right)=77.00, \delta_{\mathrm{H}}\left(\mathrm{C}_{6} \mathrm{D}_{6}\right)=128.00, \delta_{\mathrm{C}}$ $\left(\mathrm{C}_{2} \mathrm{HDCl}_{4}\right)=73.71, \delta_{\mathrm{C}}\left(\mathrm{CDCl}_{2} \mathrm{H}\right)=54.0$. Multiplizitäten wurden, soweit angegeben, mit den in der Literatur ${ }^{71}$ angegebenen Methoden bestimmt. Massenspektren und präzise Massen wurden auf einem Spektrometer Modell MAT 95 der Firma Finnigan bei 70eV aufgenommen. Spezifische Drehwerte wurden in einer $1 \mathrm{dm}$-Zelle auf einem Digital-Polarimeter 241 der Firma Perkin Elmer gemessen.

Präparative gaschromatographische Trennungen wurden mit einem Gerät GC 6000 Vega Series 2 der Firma Carlo Erba mit Wärmeleitfähigkeitsdetektor und Wasserstoff als Trägergas durchgeführt. Der Wasserstoffstrom betrug 40-80 $\mathrm{ml} / \mathrm{min}$. Die Injektor- und Detektortemperatur betrug $250^{\circ} \mathrm{C}$. Die Integrationen wurden mit einem Gerät Mega Series Integrator SP 4270 der Firma Carlo Erba durchgeführt. Responsefaktoren wurden nicht bestimmt. Analytische gaschromatographische Trennungen wurden mit einem Gerät GC 6000 Vega Series 2 der Firma Carlo Erba mit Flammenionisationsdetektor FID 40 und Split/Splitless-Injektor durchgeführt. Als Trägergas verwendeten wir Wasserstoff (60 kPa). Die Integrationen wurden mit einem Gerät Mega Series Integrator SP 4270 der Firma Carlo Erba durchgeführt. Folgende Säulen wurden verwendet: (A) $3 \mathrm{~m} \times 1 / 4^{“}$ “ Allglassystem, 15\% FFAP auf Chromosorb W AW/DMCS 60/80 mesh; (B) $3 \mathrm{~m} \times 1 \frac{1}{4}$ “ Allglassystem, 15\% OV 210 auf Chromosorb W AW/DMCS 60/80 mesh, (C) $25 \mathrm{~m} \times 0.25 \mathrm{~mm}$ (Innendurchmesser) aus deaktiviertem Quarzglas bestehende Kapillarsäule belegt mit Octakis-(2,6-di-O-pentyl-3$O$-butyryl)- $\gamma$-cyclodextrin (Lipodex ${ }^{\circledR}$ E); (D) $25 \mathrm{~m} \times 0.25 \mathrm{~mm}$ (Innendurchmesser) aus inaktiviertem Quarzglas bestehende Kapillarsäule belegt mit Octakis-(2,3-di-O-pentyl-6-Omethyl)- $\gamma$-cyclodextrin (Lipodex ${ }^{\circledR} \mathrm{G}$ ).

Dünnschichtschromatographische Untersuchungen erfolgten an DC Plastikfolien Polygram SIL G/UV 254 (Schichtdicke $0.20 \mathrm{~mm}$ ) der Firma Macherey \& Nagel. Farblose Substanzen wurden durch Besprühen mit 3.5 proz. ethanolischer 12-Molybdatophosphorsäure und anschließendes Erwärmen sichtbar gemacht. Für säulenchromatographische Trennungen stand ein Fraktionssammler Modell LINEAR II der Firma Serva Technik sowie 
ein Modell FC 220 der Firma Gilson zur Verfügung. Alle Trennungen wurden an Kieselgel (0.05-0.20 mm) durchgeführt. Angaben über die Säulenabmessungen finden sich in den jeweiligen Versuchsbeschreibungen.

Schmelzpunkte wurden auf einem Mikroheiztisch der Firma Reichert bestimmt und sind nicht korrigiert. Elementaranalysen wurden von Herrn F. Hambloch von der mikroanalytischen Abteilung des Institutes für Organische Chemie der Universität Göttingen ausgeführt.

Bäckerhefe der Firma Wieninger wurde in einem Supermarkt erworben. $\mathbf{C e C l}_{3} \cdot \mathbf{7} \mathbf{H}_{2} \mathbf{O}$ wurde zunächst bei $20^{\circ} \mathrm{C} / 15$ Torr, und dann bei $140^{\circ} \mathrm{C} / 0.1$ Torr bis zur Gewichtskonstanz entwässert und in einer dicht verschlossenen Flasche aufbewahrt. Vor jeder Anwendung wurde die eingewogene Menge nochmals $2 \mathrm{~h}$ bei $120^{\circ} \mathrm{C} / 0.1$ Torr nachgetrocknet. Wasserfreies Lithiumiodid und wasserfreies Zinkbromid wurden vor jeder Anwendung 2 h bei $120^{\circ} \mathrm{C} / 0.1$ Torr nachgetrocknet. N-Chlortoluol-4-sulfonamid (Chloramin-T) wurde im Exsikkator bei $20^{\circ} \mathrm{C} / 0.1$ Torr über $\mathrm{P}_{2} \mathrm{O}_{5}$ bis zur Gewichtskonstanz getrocknet. Wasserfreies Hydrazin wurde durch Destillation von Hydrazin-monohydrat über $\mathrm{NaOH}$ unter strömendem Argon gewonnen. ${ }^{46,72}$ Tetrahydrofuran wurde über Lithiumaluminiumhydrid 2 h unter Rückfluß erhitzt und destilliert. Diethylether wurde über KOH 2 h unter Rückfluß erhitzt und destilliert.

\section{Arbeitsvorschriften}

\section{Cyclobutyliden-cyclopentan (69)}

$\mathrm{Zu}$ einer Suspension von 4-Brombutyltriphenylphosphoniumbromid (765 g, $1.60 \mathrm{~mol}$ ) in trockenem Benzol (1.85 l) gab man unter Argon und Rühren in Abständen von 15 min portionsweise Kalium-tert-butylat ( $3 \times 119$ g, 3.20 mol). Nach beendeter Zugabe erhitzte man $3 \mathrm{~h}$ auf $50^{\circ} \mathrm{C}$, tropfte dann innerhalb 40 min Cyclopentanon (134 g, $1.60 \mathrm{~mol}$ ) zu und nach weiteren $30 \mathrm{~min}$ bei $50^{\circ} \mathrm{C}$ war die Umsetzung nach GC-Analyse beendet [Säule $\mathrm{B}, 100^{\circ} \mathrm{C}$; Retentionszeiten (min): 1.63 (Cyclopentanon), 2.61 (69)]. Man hydrolysierte mit gesättigter Ammoniumchloridlösung (110 ml), dekantierte ab, extrahierte den Rückstand mit Pentan (3 × $300 \mathrm{ml}$ ), und engte die vereinigten organischen Phasen über eine $40 \mathrm{~cm}$ Vigreuxkolonne ein (Badtemperatur bis $120^{\circ} \mathrm{C}$ ). Man verdünnte mit Pentan (300 ml), saugte vom ausgefallenen Triphenylphosphinoxid ab, wusch das Filtrat mit Wasser $(6 \times 200 \mathrm{ml})$, trocknete $\left(\mathrm{MgSO}_{4}\right)$ und engte erneut ein (Badtemperatur bis $120^{\circ} \mathrm{C}$ ). Man fraktionierte den Rückstand über eine Mikrodestillationsapparatur und erhielt so 139 g (64\%) 69 als farblose Flüssigkeit vom Sdp. $73-78^{\circ} \mathrm{C} / 30$ Torr (Reinheit $90 \%$ GC) (Lit. ${ }^{51} 155^{\circ} \mathrm{C} / 760$ Torr, $80^{\circ} \mathrm{C} / 20$ Torr). Unter vergleichbaren Bedingungen ergab ein zweiter Versuch mit 1.40 mol Cyclopentanon 122 g (71\%) 69 (Reinheit 94\% GC). Für niederaufgelöste ${ }^{1} \mathrm{H}-\mathrm{NMR}-\mathrm{Daten}$, siehe Lit. ${ }^{51}$ 
${ }^{\mathbf{1}} \mathbf{H}$-NMR (Abb. 74) (500 MHz, $\mathrm{CDCl}_{3}, \mathrm{CHCl}_{3}$ int.): $\delta=1.62 \mathrm{ppm}\left(\mathrm{m}_{\mathrm{c}}, 4 \mathrm{H}\right), 1.95$ (quint, $\mathrm{J}=8$ $\mathrm{Hz}, 2 \mathrm{H}), 2.04\left(\mathrm{~m}_{\mathrm{c}}, 4 \mathrm{H}\right), 2.57\left(\mathrm{~m}_{\mathrm{c}}, 4 \mathrm{H}\right)$.

${ }^{13}$ C-NMR (Abb. 116) (125.7 MHz, $\mathrm{CDCl}_{3}, \mathrm{CDCl}_{3}$ int.): $\delta=17.20 \mathrm{ppm}(\mathrm{t}), 26.80$ (t), 28.94 (t), $29.02(\mathrm{t}), 129.10(\mathrm{~s}), 132.82(\mathrm{~s})$.

MS (EI): $m / e=122\left(36, \mathrm{M}^{+}\right), 93(100)$.

\section{Spiro[4.4]nonan-1-on (71)}

Zu einer Lösung von 69 (71.4 g, 585 mmol) in Dichlormethan gab man unter starkem Rühren 3-Chlorperbenzoesäure (156g, 70\% w/w, $634 \mathrm{mmol}$ ) portionsweise so $\mathrm{zu}$, dass die Innentemperatur $30^{\circ} \mathrm{C}$ nicht überschritt. Anschließend versetzte man mit $1 \mathrm{~N} \mathrm{KOH} \mathrm{(1.0} \mathrm{l)} \mathrm{und}$ rührte scharf durch. Man trennte die Phasen, extrahierte die wässrige mit Dichlormethan $(2 \times$ $300 \mathrm{ml})$, wusch die die vereinigten organischen Phasen mit Wasser $(2 \times 200 \mathrm{ml})$, trocknete $\left(\mathrm{MgSO}_{4}\right)$ und filtrierte. $\mathrm{Zu}$ dem Filtrat tropfte man unter Argon und Rühren Bortrifluoridetherat (2.25 g, $15.8 \mathrm{mmol}$ ) so zu, dass die Innentemperatur $30^{\circ} \mathrm{C}$ nicht überschritt. Mit den letzten Tropfen war die exotherme Reaktion beendet. Man versetzte mit Natriumcarbonat (30 g), filtrierte $\mathrm{ab}$, engte im Rotationsverdampfer ein (Badtemperatur $50^{\circ} \mathrm{C}$ ) und fraktionierte den Rückstand über eine Mikrodestille. Man erhielt so 73.6 g (97\%) 71 als farblose Flüssigkeit vom Sdp. $85-88^{\circ} \mathrm{C} / 16$ Torr [Reinheit $96 \%$ GC, Säule A, $160^{\circ} \mathrm{C}$; Retentionszeit (min): 5.75

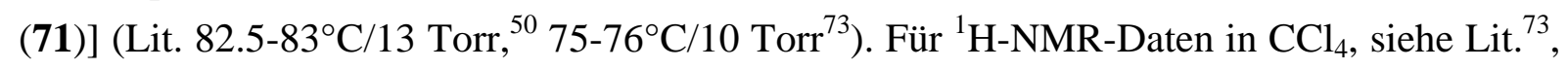
für ${ }^{13} \mathrm{C}$-NMR-Daten siehe Lit. $^{74}$

${ }^{1} \mathbf{H}-\mathbf{N M R}$ (Abb. 75) (500 MHz, $\mathrm{CDCl}_{3}, \mathrm{CHCl}_{3}$ int.): $\delta=1.33-1.41 \mathrm{ppm}$ (m, 2H), 1.50-1.60 (m, 2H), 1.60-1.73 (m, 4H), 1.73-1.82 (m, 4H), 2.16 (t, J = $7 \mathrm{~Hz}, 2 \mathrm{H})$.

${ }^{13}$ C-NMR (Abb. 117) (125.7 MHz, $\mathrm{CDCl}_{3}, \mathrm{CDCl}_{3}$ int): $\delta=19.52 \mathrm{ppm}(\mathrm{t}), 25.73(\mathrm{t}), 32.64(\mathrm{t})$, $37.28(\mathrm{t}), 38.02(\mathrm{t}), 56.14(\mathrm{~s}), 223.89(\mathrm{~s})$.

\section{1-Methylen-spiro[4.4]nonan (25)}

Zu einer Suspension von Methyltriphenylphosphoniumbromid (221 g, $619 \mathrm{mmol}$ ) in wasserfreiem Ether (1.25 l) gab man unter Argon und Rühren Kalium-tert-butylat (69.3 g, 619 mmol) und erhitzte unter Rückfluss. Nach 30 min destillierte man alles bis zu einer Badtemperatur von $60^{\circ} \mathrm{C}$ Flüchtige ab und tropfte anschließend 71 (71.1 g, $516 \mathrm{mmol}$ ) zu. Nach weiteren $1.5 \mathrm{~h}$ bei $60^{\circ} \mathrm{C}$ war der Umsatz laut GC komplett [Säule A, $160^{\circ} \mathrm{C}$, Retentionszeiten (min): 1.63 (25), 5.57 (71)]. Man verdünnte mit Pentan (250 ml), hydrolysierte mit Wasser (50 ml), dekantierte die organische Phase ab und extrahierte den Rückstand mit Pentan (3 × $250 \mathrm{ml}$ ). Man wusch die vereinigten organischen Phasen mit Wasser $(5 \times 250 \mathrm{ml})$, trocknete $\left(\mathrm{MgSO}_{4}\right)$, destillierte die Lösungsmittel über eine $40 \mathrm{~cm}$ Vigreuxkolonne ab (Badtemperatur bis $120^{\circ} \mathrm{C}$ ) und fraktionierte den Rückstand über eine Mikrodestille. Man erhielt so $66.3 \mathrm{~g}$ 
(95\%) 25 als farblose Flüssigkeit vom Sdp. 79-81C/14 Torr (Reinheit 97\% GC) (Lit.: $162^{\circ} \mathrm{C} / 760$ Torr, ${ }^{19} 78^{\circ} \mathrm{C} / 25$ Torr ${ }^{20}$ ). Die ${ }^{1} \mathrm{H}^{19,22}$ und ${ }^{13} \mathrm{C}-\mathrm{NMR}-D a t e{ }^{22}$ stimmten mit Literaturdaten überein.

\section{1-(Cyclopentylcarbonyl)-piperidin (23)}

$\mathrm{Zu}$ einer Lösung von Piperidin (32.2 g, $378 \mathrm{mmol})$ in Dichlormethan (140 ml) tropfte man unter Argon und Rühren sowie zeitweiliger Kühlung mit Eis Cyclopentancarbonsäurechlorid (25.0 g, $189 \mathrm{mmol}$ ) so zu, dass die Innentemperatur $20^{\circ} \mathrm{C}$ nicht überschritt. Anschließend hielt man noch 30 min bei Raumtemperatur, wusch anschließend mit Wasser (80 ml), 2 N HCl (80 ml) und gesättigter Natriumhydrogencarbonatlösung $(80 \mathrm{ml})$, und trocknete $\left(\mathrm{MgSO}_{4}\right)$. Man engte im Rotationsverdampfer ein und fraktionierte den Rückstand im Hochvakuum. Man erhielt so 33.0 g (96\%) 23 als farblose Flüssigkeit vom Sdp. $111^{\circ} \mathrm{C} / 0.35$ Torr [Reinheit $>99 \%$ GC, Säule A, $230^{\circ} \mathrm{C}$; Retentionszeit (min): 5.82 (23)].

$\mathrm{C}_{11} \mathrm{H}_{19} \mathrm{NO}(181.1)$

Ber. C $72.88 \quad$ H 10.56

Gef. C $73.11 \quad$ H 10.47

IR (Abb. 12) (Film): $1640 \mathrm{~cm}^{-1}(\mathrm{C}=\mathrm{O})$

${ }^{1}$ H-NMR (Abb. 49) (500 MHz, $\mathrm{CDCl}_{3}, \mathrm{CHCl}_{3}$ int.): $\delta=1.36-1.51 \mathrm{ppm}$ (m, 6H), 1.51-1.58 (m, 2H), 1.58-1.67 (m, 2H), 1.67-1.76 (m, 4H), 2.80 (tt, J = 8 Hz, $8 \mathrm{~Hz}, 1 \mathrm{H}), 3.34\left(\mathrm{~m}_{\mathrm{c}}, 2 \mathrm{H}\right)$, $3.46\left(\mathrm{~m}_{\mathrm{c}}, 2 \mathrm{H}\right)$.

${ }^{13}$ C-NMR (Abb. 90) (150.8 MHz, $\mathrm{CDCl}_{3}, \mathrm{CDCl}_{3}$ int.): $\delta=24.51 \mathrm{ppm}(\mathrm{t}), 25.45$ (t), 25.81 (t), $26.48(\mathrm{t}), 29.92(\mathrm{t}), 40.87(\mathrm{~d}), 42.65(\mathrm{t}), 46.31(\mathrm{t}), 174.01(\mathrm{~s})$.

MS (EI): $m / e=181\left(29, \mathrm{M}^{+}\right), 140(100)$.

\section{$\left(5 R^{*}\right)$-Trispiro[3.0.0.4.2.2]tetradecan-11-on [(5R*)-24]}

$\mathrm{Zu}$ einer Lösung von 23 (15.1 g, $83.5 \mathrm{mmol})$ in Dichlormethan $(80 \mathrm{ml})$ gab man bei $-15^{\circ} \mathrm{C}$ unter Argon und Rühren zunächst Trifluormethansulfonsäureanhydrid (28.3 g, $100 \mathrm{mmol}$ ) und dann, innerhalb 15 min, ein Gemisch von 2,4,6-Collidin (13.1 g, 108 mmol) und 25 (17.9 g, 166 mmol). Man erhitzte 20 h unter Rückfluß, engte anschließend im Rotationsverdampfer ein (Badtemperatur $40^{\circ} \mathrm{C} / 20$ Torr) und extrahierte den Rückstand mit Ether (6 × $40 \mathrm{ml}$ ). Man engte erneut ein, nahm das zurückgebliebene braune Öl in Dichlormethan (170 ml) auf und hydrolysierte mit Wasser (170 ml). Nach 2 h Rückfluß trennte man die Phasen, extrahierte die wässrige mit Dichlormethan $(6 \times 60 \mathrm{ml})$, wusch die vereinigten organischen Phasen mit gesättigter Ammoniumchloridlösung $(60 \mathrm{ml})$ und trocknete $\left(\mathrm{K}_{2} \mathrm{CO}_{3} / \mathrm{MgSO}_{4}\right)$. Man engte im Rotationsverdampfer ein (Badtemperatur $40^{\circ} \mathrm{C} / 20$ Torr), extrahierte den Rückstand (26.1 g) 
mit Pentan/Ether 1:1 $(3 \times 50 \mathrm{ml})$, wusch die vereinigten Extrakte mit $2 \mathrm{~N} \mathrm{HCl}(30 \mathrm{ml})$, trocknete $\left(\mathrm{MgSO}_{4}\right)$, engte erneut ein und chromatographierte den Rückstand (9.4 g) an Kieselgel in Pentan/Ether 9:1 (Säule $75 \times 5 \mathrm{~cm}, \mathrm{R}_{\mathrm{f}}=0.45$ [(5R*)-24]). Man erhielt $3.63 \mathrm{~g}$ (21\%) (5R*)-24 als farblose Flüssigkeit (Reinheit 83\% GC, Säule A, $230^{\circ} \mathrm{C}$, Retentionszeit (min): 5.11 [(5R*)-24]). Für die Reduktion mit Bäckerhefe wurde das Material nicht weiter gereinigt. Für die Darstellung von 13, sowie von 77 und 78 wurde es zweimal chromatographiert (Reinheit 95\% GC). Eine analytisch reine Probe wurde durch präparative GC erhalten.

$$
\mathbf{C}_{14} \mathbf{H}_{22} \mathbf{O} \text { (204.3) }
$$

Ber.: C $82.30 \quad$ H 9.87

Gef.: C 81.99 H 9.72

IR (Abb. 13) (Film): $1770 \mathrm{~cm}^{-1}(\mathrm{C}=\mathrm{O})$.

${ }^{1} \mathbf{H}-\mathbf{N M R}$ (Abb. 50) (600 MHz, $\mathrm{CDCl}_{3}, \mathrm{CHCl}_{3}$ int.): $\delta=1.54-1.78$ ppm (m, 9H), 1.78-1.93 (m, 5H), 1.97-2.04 (m, 2H), $2.10\left(\mathrm{~m}_{\mathrm{c}}, 1 \mathrm{H}\right), 2.28\left(\mathrm{~m}_{\mathrm{c}}, 1 \mathrm{H}\right), 2.62$ (d, J = 16.5 Hz, 1H), 3.12 (d, J $=16.5 \mathrm{~Hz}, 1 \mathrm{H})$.

${ }^{13}$ C-NMR (Abb. 91) (75.5 MHz, $\mathrm{CDCl}_{3}, \mathrm{CDCl}_{3}$ int.): $\delta=15.43$ ppm (t), 25.24 (t), 25.97 (t), $26.27(\mathrm{t}), 30.03(\mathrm{t}), 30.97(\mathrm{t}), 31.33(\mathrm{t}), 32.53(\mathrm{t}), 44.55(\mathrm{~s}), 48.65(\mathrm{~s}), 51.65(\mathrm{t}), 71.77(\mathrm{~s})$, $214.10(\mathrm{~s})$.

MS (EI): $m / e=204\left(<1, \mathrm{M}^{+}\right), 134(100)$.

$\left(1 R^{*}\right)-1-C y c l o p e n t y l-2-s p i r o[4.4]$ non-1-yl-ethanon $\left[\left(1 R^{*}\right)-75\right]$ und 1-Cyclopentyl-2spiro[4.4]non-1-en-1-yl-ethanon (76)

Zu einer Lösung von 23 (1.80 g, $10.0 \mathrm{mmol})$ in Dichlormethan $(10 \mathrm{ml})$ gab man bei $-15^{\circ} \mathrm{C}$ innerhalb 15 min unter Argon und Rühren zunächst Trifluormethansulfonsäureanhydrid (3.40 g, $12.0 \mathrm{mmol}$ ) und dann, innerhalb $30 \mathrm{~min}$ ein Gemisch von 25 (2.80 g, $21.0 \mathrm{mmol}$ ) und 2,4,6-Collidin (1.50 g, 12.0 mmol). Anschließend erhitzte man 16 h unter Rückfluß. Man engte im Rotationsverdampfer ein (Badtemperatur $40^{\circ} \mathrm{C} / 20$ Torr), extrahierte den Rückstand mit Ether $(8 \times 8 \mathrm{ml})$, engte erneut ein und hydrolysierte das zurückgebliebene braune Öl nach Aufnahme in Dichlormethan $(20 \mathrm{ml})$ mit Wasser $(20 \mathrm{ml})$. Nach 2 h unter Rückfluß trennte man die Phasen, extrahierte die wässrige Phase mit Dichlormethan $(5 \times 10 \mathrm{ml})$, trocknete die vereinigten organischen Phasen $\left(\mathrm{K}_{2} \mathrm{CO}_{3} / \mathrm{MgSO}_{4}\right)$ und engte wiederum ein. Man extrahierte den Rückstand mit Pentan/Ether 1:1 (3 × $10 \mathrm{ml})$, engte erneut ein und chromatographierte den Rückstand an Kieselgel in Pentan/Ether 93:7 [Säule $88 \times 2.5 \mathrm{~cm}, \mathrm{R}_{\mathrm{f}}=0.44$ ([(1R*)-75]), 0.39 (76)]. Man erhielt so $30 \mathrm{mg}$ (1\%) (1R*)-75 und $82 \mathrm{mg}$ (3\%) 76 als leicht gelbliche Flüssigkeiten. 


\section{$\left(1 R^{*}\right)-1-C y c l o p e n t y l-2-s p i r o[4.4]$ non-1-yl-ethanon [(1R*)-75]}

IR (Abb. 36) (Film): $1720 \mathrm{~cm}^{-1}(\mathrm{C}=\mathrm{O})$.

${ }^{1} \mathbf{H}-\mathbf{N M R}$ (Abb. 76) (600 MHz, $\mathrm{CDCl}_{3}, \mathrm{CHCl}_{3}$ int.): $\delta=1.06-1.17 \mathrm{ppm}(\mathrm{m}, 1 \mathrm{H}), 1.17-1.25$ (m, 1H), 1.25-1.33 (m, 1H), 1.33-1.46 (m, 3H), 1.46-1.69 (m, 12H), 1.69-1.84 (m, 4H), 2.07 (m $\mathrm{c}, 1 \mathrm{H}), 2.23$ (dd, $\mathrm{J}=15,10 \mathrm{~Hz}, 1 \mathrm{H}), 2.44$ (dd, $\mathrm{J}=15,3 \mathrm{~Hz}, 1 \mathrm{H}), 2.84$ (tt, $\mathrm{J}=$ 7.5, $7.5 \mathrm{~Hz}$, $1 \mathrm{H})$.

${ }^{13}$ C-NMR (Abb. 118) (150.8 MHz, $\mathrm{CDCl}_{3}, \mathrm{CDCl}_{3}$ int.): $\delta=21.49$ ppm (t), 24.38 (t), 24.79 $(\mathrm{t}), 25.93(\mathrm{t}), 25.99(\mathrm{t}), 28.73(\mathrm{t}), 29.16(\mathrm{t}), 31.05(\mathrm{t}), 32.25(\mathrm{t}), 37.42(\mathrm{t}), 38.92(\mathrm{t}), 42.65(\mathrm{~d})$, 43.49 (t), 51.64 (d), 52.77 (s), 213.80 (s).

\section{1-Cyclopentyl-2-spiro[4.4]non-1-en-1-yl-ethanon (76)}

IR (Abb. 37) (Film): $1720 \mathrm{~cm}^{-1}(\mathrm{C}=\mathrm{O}), 1625$ (C=C).

${ }^{1} \mathbf{H}-\mathbf{N M R}$ (Abb. 77) (600 MHz, $\mathrm{CDCl}_{3}, \mathrm{CHCl}_{3}$ int.): $\delta=1.31-1.37 \mathrm{ppm}(\mathrm{m}, 2 \mathrm{H}), 1.38-1.46$ (m, 2H), 1.50-1.80 (m, 14H), $2.21\left(\mathrm{~m}_{\mathrm{c}}, 2 \mathrm{H}\right), 2.97$ (tt, J = 8, $\left.8 \mathrm{~Hz}, 1 \mathrm{H}\right), 3.04\left(\mathrm{~m}_{\mathrm{c}}, 2 \mathrm{H}\right), 5.40$ $\left(\mathrm{m}_{\mathrm{c}}, 1 \mathrm{H}\right)$.

${ }^{13}$ C-NMR (Abb. 119) (150.8 MHz, $\mathrm{CDCl}_{3}, \mathrm{CDCl}_{3}$ int.): $\delta=24.46$ ppm (t), 26.01 (t), 29.25 (t), $29.69(\mathrm{t}), 36.11(\mathrm{t}), 39.12$ (t), 40.83 (t), 50.43 (d), 57.67 (s), 127.40 (d), 142.33 (s), 212.12 (s).

\section{$\left(5 R^{*}\right)$-Trispiro[3.0.0.4.2.2] tetradecan $\left[\left(5 R^{*}\right)-13\right]$}

$\mathrm{Zu}$ einer Lösung von Hydrazinhydrat (150 mg, $3.0 \mathrm{mmol}$ ) und gepulvertem KOH (225 mg, $4.0 \mathrm{mmol}$ ) in Diethylenglykol (2.0 ml) gab man unter Argon und Rühren (5R*)-24 (102 mg, $0.50 \mathrm{mmol}$ ). Die Reaktionsmischung wurde $1.5 \mathrm{~h}$ auf $160^{\circ} \mathrm{C}$ erhitzt, anschließend mit Wasser $(20 \mathrm{ml})$ verdünnt und mit Pentan $(3 \times 15 \mathrm{ml})$ extrahiert. Die vereinigten Extrakte wurden mit Wasser $\left(15 \mathrm{ml}\right.$ ) gewaschen, über $\mathrm{MgSO}_{4}$ getrocknet und über eine $20 \mathrm{~cm}$ Vigreux-Kolonne eingeengt. Letzte Reste Lösungsmittel entfernte man unter vermindertem Druck. Es verblieben $88 \mathrm{mg}(82 \%)\left(5 R^{*}\right)-\mathbf{1 3}$ als farblose Flüssigkeit (Reinheit $99 \%$ GC, Säule A, $180^{\circ} \mathrm{C}$; Retentionszeit (min): 3.69 [(5R*)-13]). Die Enantiomeren wurden kapillargaschromatographisch an einer chiralen Phase differenziert [Säule D, $90^{\circ} \mathrm{C}$; Retentionszeiten (min): 32.85/33.42]. Eine analytisch reine Probe des Racemats wurde durch präparative GC gewonnen.

$\mathbf{C}_{14} \mathbf{H}_{22}$ (190.3)

Ber.: C $88.35 \quad$ H 11.65

Gef.: C $88.01 \quad$ H 11.45

IR (Abb. 9) (Film) 
${ }^{1}$ H-NMR (Abb. 46) (600 MHz, $\mathrm{CDCl}_{3}, \mathrm{CHCl}_{3}$ int.): $\delta=1.35-1.40$ ppm (m, 1H), 1.40-1.65 (m, 11H), 1.68-1.87 (m, 6H), $1.93\left(\mathrm{~m}_{\mathrm{c}}, 1 \mathrm{H}\right), 1.99\left(\mathrm{~m}_{\mathrm{c}}, 1 \mathrm{H}\right), 2.09\left(\mathrm{~m}_{\mathrm{c}}, 1 \mathrm{H}\right), 2.23-2.30(\mathrm{~m}, 1 \mathrm{H})$.

${ }^{13}$ C-NMR (Abb. 87) (75.5 MHz, $\mathrm{CDCl}_{3}, \mathrm{CDCl}_{3}$ int.): $\delta=15.84$ ppm (t), 23.14 (t), 24.32 (t), $26.63(\mathrm{t}), 27.09(\mathrm{t}), 30.43(\mathrm{t}), 31.11(\mathrm{t}), 32.07(\mathrm{t}), 32.20(\mathrm{t}), 33.35(\mathrm{t}), 36.09(\mathrm{t}), 49.02(\mathrm{~s})$, 51.89 (s), 52.08 (s).

MS (EI): $m / e=190\left(<1, \mathrm{M}^{+}\right), 162\left(38, \mathrm{M}^{+}-\mathrm{C}_{2} \mathrm{H}_{4}\right), 79(100)$.

(5R*)-Trispiro[3.0.0.4.3.2]tetradecan-12-on [(5R*)-77] und (5R*)-Trispiro[3.0.0.4.3.2]tetradecan-11-on [(5R*)-78)]

Zu einer Lösung von (5R*)-24 (592 mg, $2.90 \mathrm{mmol}$ ) und gepulvertem Kaliumhydroxid (1.94 g, $34.7 \mathrm{mmol})$ in Methanol $(4.4 \mathrm{ml})$ und Wasser $(0.7 \mathrm{ml})$ gab man portionsweise innerhalb von 25 min N-Methyl-N-nitroso-4-toluolsulfonsäureamid (Diazald ${ }^{\circledR}$ ) (806 mg, 3.76 mmol). Anschließend verdünnte man mit Methanol (3.0 ml) und gab nach 45 min zunächst weiteres Diazald $^{\circledR}$ (820 mg, $3.83 \mathrm{mmol}$ ) und dann weiteres Methanol (3.0 ml) zu. Nach einer weiteren Stunde enthielt die Mischung laut GC (Säule A, $230^{\circ} \mathrm{C}$, Retentionszeiten (min): 4.88 [(5R*)24], 7.86 [(5R*)-78], 10.13 [(5R*)-77]) ein 55:45-Gemisch von (5R*)-77 und (5R*)-78. Die heterogene Mischung wurde mit Wasser $(8 \mathrm{ml})$ verdünnt und die dann klare Lösung mit Pentan $(7 \times 10 \mathrm{ml})$ extrahiert. Die vereinigten Extrakte wurden mit gesättigter Ammoniumchloridlösung $(15 \mathrm{ml})$ und Wasser $(15 \mathrm{ml})$ gewaschen, getrocknet $\left(\mathrm{MgSO}_{4}\right)$, und im Rotationsverdampfer eingeengt $\left(40^{\circ} \mathrm{C} / 15\right.$ Torr). Man erhielt so $449 \mathrm{mg}$ (71\%) eines 55:45Gemisches von $\left(5 R^{*}\right)-77$ und $\left(5 R^{*}\right)-78$ als farblose Flüssigkeit (Reinheit 93\% GC). Das Gemisch wurde für die Darstellung von $\left(5 R^{*}\right)$-14 eingesetzt. Analytisch reine Proben von $\left(5 R^{*}\right)$-77 und $\left(5 R^{*}\right)$-78 wurden als farblose Flüssigkeiten durch präparative GC erhalten.

$\mathbf{C}_{15} \mathbf{H}_{22} \mathbf{O}(218.3)$

Ber.: C $82.52 \quad$ H 10.16

Gef.: C $82.42 \quad$ H 9.82

\section{$\left(5 R^{*}\right)$-Trispiro[3.0.0.4.3.2] tetradecan-12-on [(5R*)-77]}

IR (Abb. 38) (Film): $1750 \mathrm{~cm}^{-1}(\mathrm{C}=\mathrm{O})$.

${ }^{1} \mathbf{H}-\mathbf{N M R}$ (Abb. 78) (600 MHz, $\mathrm{CDCl}_{3}, \mathrm{CHCl}_{3}$ int.): $\delta=1.35-1.40 \mathrm{ppm}$ (m, 1H), 1.41-1.46 (m, 1H), 1.50-1.89 (m, 12H), 1.92-2.08 (m, 3H), 1.95 (d, J = $18 \mathrm{~Hz}, 1 \mathrm{H}), 2.16$ (d, J = $18 \mathrm{~Hz}$, 1H), 2.18 (d, J = $18 \mathrm{~Hz}, 1 \mathrm{H}), 2.31-2.38$ (m, 1H), 2.37 (d, J = $18 \mathrm{~Hz}, 1 \mathrm{H}$ ).

${ }^{13}$ C-NMR (Abb. 120) (150.8 MHz, $\mathrm{CDCl}_{3}, \mathrm{CDCl}_{3}$ int.): $\delta=15.68 \mathrm{ppm}(\mathrm{t}), 24.48$ (t), 25.11 $(\mathrm{t}), 25.21(\mathrm{t}), 32.02(\mathrm{t}), 32.30(\mathrm{t}), 32.92(\mathrm{t}), 33.21(\mathrm{t}), 34.92(\mathrm{t}), 47.79(\mathrm{t}), 48.34(\mathrm{~s}), 51.64(\mathrm{~s})$, $52.50(\mathrm{t}), 53.01(\mathrm{~s}), 218.85(\mathrm{~s})$.

MS (EI): $m / e=218\left(29, \mathrm{M}^{+}\right), 122(100)$. 


\section{$\left(5 R^{*}\right)$-Trispiro[3.0.0.4.3.2] tetradecan-11-on [(5R*)-78]}

IR (Abb. 39) (Film): $1740 \mathrm{~cm}^{-1}$ (C=O).

${ }^{1}$ H-NMR (Abb. 79) (600 MHz, $\mathrm{CDCl}_{3}, \mathrm{CHCl}_{3}$ int.): $\delta=1.50-1.55$ ppm (m, 2H), 1.57-1.74 (m, 12H), 1.76-1.85 (m, 2H), 1.91-2.00 (m, 2H), 2.01-2.09 (m, 2H), 2.21 (pseudo t, J = 7.5 $\mathrm{Hz}, 2 \mathrm{H})$.

${ }^{13}$ C-NMR (Abb. 121) (75.5 MHz, $\mathrm{CDCl}_{3}, \mathrm{CDCl}_{3}$ int.): $\delta=15.81 \mathrm{ppm}(\mathrm{t}), 24.23(\mathrm{t}), 25.80(\mathrm{t})$, $26.50(\mathrm{t}), 28.50(\mathrm{t}), 29.53(\mathrm{t}), 31.46(\mathrm{t}), 32.15(\mathrm{t}), 32.78(\mathrm{t}), 33.05(\mathrm{t}), 33.92(\mathrm{t}), 48.07(\mathrm{~s})$, 52.42 (s), 62.30 (s), 221.36 (s).

MS (EI): $m / e=218\left(6, \mathrm{M}^{+}\right), 41(100)$.

\section{$\left(5 R^{*}\right)$-Trispiro[3.0.0.4.3.2]pentadecan $\left[\left(5 R^{*}\right)-14\right]$}

Zu einer Lösung von Hydrazinhydrat (446 mg, $8.9 \mathrm{mmol}$ ) und gepulvertem Kaliumhydroxid (670 mg, $11.9 \mathrm{mmol})$ in Diethylenglykol $(7.0 \mathrm{ml}$ ) gab man unter Argon und Rühren ein 55:45-Gemisch von (5R*)-77 und (5R*)-78 (325 mg, $1.49 \mathrm{mmol}$ ) und erhitzte $2 \mathrm{~h}$ auf $120^{\circ} \mathrm{C}$ und $5 \mathrm{~h}$ auf $195^{\circ} \mathrm{C}$. Man verdünnte mit Wasser $(10 \mathrm{ml})$, extrahierte mit Pentan $(6 \times 10 \mathrm{ml})$, wusch die vereinigten Extrakte mit gesättigter Ammoniumchloridlösung (15 ml) und Wasser (15 ml), und trocknete $\left(\mathrm{MgSO}_{4}\right)$. Man destillierte das Lösungsmittel über eine $20 \mathrm{~cm}$ VigreuxKolonne $(20 \mathrm{~cm})$ ab und chromatographierte den Rückstand an Kieselgel in Pentan (Säule 45 $\left.\times 3 \mathrm{~cm}, \mathrm{R}_{\mathrm{f}}=0.77\left[\left(5 R^{*}\right)-14\right]\right)$. Man erhielt so $187 \mathrm{mg}(66 \%)\left(5 R^{*}\right)-14$ als farblose Flüssigkeit (Reinheit 87\% GC). Eine analytisch reine Probe wurde durch präparative GC erhalten (Säule A, $180^{\circ} \mathrm{C}$; Retentionszeit (min): 6.64 [(5R*)-14]). Die Enantiomeren konnten nicht differenziert werden [Säule D, $110^{\circ} \mathrm{C}$, Retentionszeit (min): $21.09\left(5 R^{*} / 5 S^{*}\right)-14$ ].

$\mathbf{C}_{15} \mathbf{H}_{24}(204.4)$

Ber.: C 88.16 H 11.84

Gef.: C $88.02 \quad$ H 11.79

IR (Abb. 10) (Film)

${ }^{1} \mathbf{H}-\mathbf{N M R}$ (Abb. 47) (600 MHz, $\mathrm{CDCl}_{3}, \mathrm{CHCl}_{3}$ int.): $\delta=1.19-1.26$ ppm (m, 1H), 1.28-1.37 (m, 2H), 1.39-1.64 (m, 13H), 1.67-1.81 (m, 4H), 1.88-1.96 (m, 2H), $1.99\left(\mathrm{~m}_{\mathrm{c}}, 1 \mathrm{H}\right), 2.28\left(\mathrm{~m}_{\mathrm{c}}, 1 \mathrm{H}\right)$.

${ }^{13}$ C-NMR (Abb. 88) (150.8 MHz, $\mathrm{CDCl}_{3}, \mathrm{CDCl}_{3}$ int.): $\delta=15.96$ ppm (t), $19.44(\mathrm{t}), 24.90(\mathrm{t})$, $25.17(\mathrm{t}), 25.64(\mathrm{t}), 32.21(\mathrm{t}), 32.22(\mathrm{t}), 32.78(\mathrm{t}), 33.41(\mathrm{t}), 33.56(\mathrm{t}), 34.66(\mathrm{t}), 38.24(\mathrm{t}), 48.79$ (s), 53.96 (s), 55.06 (s).

MS (EI): $m / e=204\left(4, \mathrm{M}^{+}\right), 121(100)$. 
Reduktion von (5R*)-24 mit Lithiumaluminiumhydrid: $\left(5 R^{*}, 11 R^{*}\right)$-Trispiro[3.0.0.4.2.2]tetradecan-11-ol [(5R*,11 $\left.\left.R^{*}\right)-28\right]$ und $\left(5 R^{*}, 11 S^{*}\right)$-Trispiro[3.0.0.4.2.2]tetradecan-11-ol $\left[\left(5 R^{*}, 11 S^{*}\right)-29\right]$

Zu einer Suspension von Lithiumaluminiumhydrid (114 mg, $3.0 \mathrm{mmol}$ ) in wasserfreiem Ether (8.0 ml) wurde bei Raumtemperatur unter Argon und Rühren eine Lösung von (5R*)-24 (204 $\mathrm{mg}, 1.0 \mathrm{mmol})$ in wasserfreiem Ether $(1.5 \mathrm{ml})$ zugegeben. Nach $30 \mathrm{~min}$ war die Reduktion nach GC-Analyse beendet (Säule A, $230^{\circ} \mathrm{C}$; Retentionszeiten (min): 5.11 [(5R*)-24], 7.22

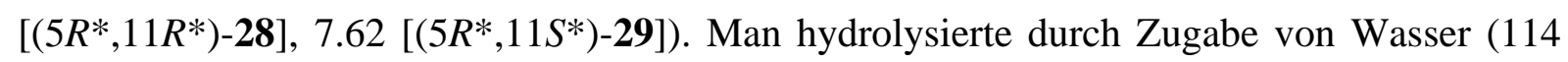
$\mu \mathrm{l}), 15 \%$ Kaliumhydroxidlösung $(114 \mu \mathrm{l})$ und Wasser (342 $\mu \mathrm{l})$, dekantierte die überstehende Lösung ab und extrahierte den Rückstand mit Ether $(3 \times 10 \mathrm{ml})$. Die vereinigten organischen Phasen wurden getrocknet $\left(\mathrm{MgSO}_{4}\right)$ und im Rotationsverdampfer eingeengt (Badtemperatur $45^{\circ} \mathrm{C} / 20$ Torr). Es verblieben $200 \mathrm{mg}$ eines $65: 35$-Gemisches von $\left(5 R^{*}, 11 R^{*}\right)$-28 und $\left(5 R^{*}, 11 S^{*}\right)-29$. Die Alkohole waren weder an Kieselgel noch über präparative GC trennbar. Allerdings konnten die Enantiomere von $\left(5 R^{*}, 11 R^{*}\right)$-28 differenziert werden [Säule $\mathrm{C}$, $130^{\circ} \mathrm{C}$; Retentionszeiten (min): 27.02 (5R,11R)-28, 27.45 (5S,11S)-28, 30.07 (5R,11S)- und (5S,11R)-29]. Alle Zuordnungen beruhen auf den Ergebnissen der Reduktion mit Bäckerhefe (s. unten). Die ${ }^{1} \mathrm{H}$ - und ${ }^{13} \mathrm{C}-\mathrm{NMR}$-Daten des Gemisches stimmen mit denen der reinen Alkohole überein.

$\mathbf{C}_{14} \mathbf{H}_{22} \mathbf{O}$ (206.3)

Ber.: C $81.50 \quad$ H 10.75

Gef.: C $81.38 \quad$ H 10.81

Reduktion von (5R*)-24 mit Bäckerhefe: (5S,11S)-(+)-Trispiro[3.0.0.4.2.2]tetradecan-11ol [(5S,11S)-(+)-28)], (5R,11S)-(+)-Trispiro[3.0.0.4.2.2]tetradecan-11-ol [(5R,11S)-(+)-29)] und (5R)-(-)-Trispiro[3.0.0.4.2.2] tetradecan-11-on [(5R)-(-)-24]

$\mathrm{Zu}$ einer Mischung aus frischer Bäckerhefe (40 g), Saccharose (40 g) und Wasser (400 ml) gab man eine Lösung von (5R*)-24 (3.25 g, Reinheit 83\%, $13.3 \mathrm{mmol})$ in Ethanol (13.5 ml) und rührte anschließend bei $35^{\circ} \mathrm{C}$. Man überwachte die Reaktion durch GC (Säule A, $230^{\circ} \mathrm{C}$;

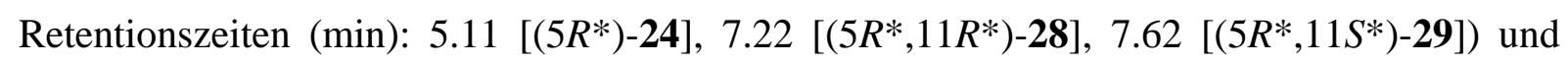
gab nach 8 h weitere Bäckerhefe (40 g), Saccharose (40 g) und Wasser (200 ml) zu. Nach $22 \mathrm{~h}$ verdünnte man mit Wasser (4.5 l) und extrahierte kontinuierlich mit Ether $(1.0 \mathrm{l}, 6 \mathrm{~h}$, GC-Kontrolle). Der Extrakt wurde auf $150 \mathrm{ml}$ eingeengt, mit gesättigter Natriumhydrogencarbonatlösung $(2 \times 30 \mathrm{ml})$ gewaschen, getrocknet $\left(\mathrm{MgSO}_{4}\right)$ und eingeengt (Badtemperatur $50^{\circ} \mathrm{C} / 20$ Torr). Nach GC-Analyse enthielt der ölige Rückstand (3.46 g) 24, (5S,11S)-28 und (5R,11S)-29 im Verhältnis von 58:40:2. Damit bestand 24 aus einem 83:17-Gemisch von (5R)- und (5S)-24. Das gesamte Material wurde an Kieselgel in Pentan/Ether 7:3 chromatographiert \{Säule $80 \times 5 \mathrm{~cm}, \mathrm{R}_{\mathrm{f}}=0.65$ (24), 0.35 ([(5S,11S)-28]/[(5R,11S)-29]\} und lieferte 
1.44 g (53\%) 24 als farblose Flüssigkeit (Reinheit 90\%), und 919 mg (34\%) eines 96:4Gemisches von (5S,11S)-28 und (5R,11S)-29 als farblosen Feststoff vom Schmp. $47-52^{\circ} \mathrm{C}$ (Reinheit $98 \%,[\alpha]_{\mathrm{D}}{ }^{20}=+33.6$, c 1.13 , Aceton). Nach der GC-Analyse an einer chiralen Phase [Säule C, $130^{\circ} \mathrm{C}$; Retentionszeit (min): 27.45] war (5S,11S)-28 enantiomerenrein (>99\% ee). Die Reduktion des 83:17-Gemisches von (5R)- und (5S)-24 (1.44 g, Reinheit 90\%, 6.35 mmol) in einer Mischung aus Bäckerhefe (19 g), Saccharose (19 g) und Wasser (190 ml) wurde analog durchgeführt. Nach 7, 24, 31, 48 und 72 h wurde weitere Bäckerhefe (19 g), Saccharose (19 g) und Wasser (95 ml) zugegeben, und nach $96 \mathrm{~h}$ enthielt der Extrakt (1.26 g) 24, (5S,11S)-28 und (5R,11S)-29 im Verhältnis von 78:16:12. Damit bestand 24 aus einem 99:1-Gemisch von (5R)- und (5S)-24. Säulenchromatographie lieferte 810 mg (62\%) 24. Davon wurden 786 mg (Reinheit 92\%, 3.54 mmol) einer dritten Reduktion mit Bäckerhefe (11 g), Saccharose (11 g) und Wasser (110 ml) unterworfen. Nach 6, 23 und $30 \mathrm{~h}$ wurde weitere Bäckerhefe (11 g), Saccharose (11 g) und Wasser (55 ml) zugegeben, und nach $48 \mathrm{~h}$ enthielt der Extrakt (811 mg) 24, (5S,11S)-28 und (5R,11S)-29 im Verhältnis von 88:0.5:11.5. Damit bestand 24 aus enantiomerenreinem (5R)-24. Säulenchromatographie lieferte $564 \mathrm{mg}$ (78\%) (5R)-24 als farblose Flüssigkeit (Reinheit 92\%) und $73 \mathrm{mg}$ (10\%) eines 95:5Gemisches von $(5 R, 11 S)$-29 und $(5 S, 11 S)$-28 als farblosen Feststoff vom Schmp. $78-82^{\circ} \mathrm{C}$ (Reinheit 96\%). Analytisch reine Proben von $(5 R)-24\left([\alpha]_{\mathrm{D}}{ }^{20}=-0.5\right.$, c 1.13 , Aceton) und dem 95:5-Gemisch von $(5 R, 11 S)-29$ und $(5 S, 11 S)-28\left([\alpha]_{D}{ }^{20}=+62.5\right.$, с 1.13 , Aceton) wurden durch präparative GC erhalten. Die ${ }^{1} \mathrm{H}$ - und ${ }^{13} \mathrm{C}$-NMR-Daten von (5R)-24 waren mit denen des Racemats identisch.

\section{$(5 S, 11 S)-(+)-T r i s p i r o[3.0 .0 .4 .2 .2]$ tetradecan-11-ol [(5S,11S)-(+)-28)]}

IR (Abb. 14) (KBr): $3120-3560 \mathrm{~cm}^{-1}\left(\mathrm{OH}_{\mathrm{ass}}\right)$.

${ }^{1}$ H-NMR (Abb. 51) (600 MHz, $\mathrm{CDCl}_{3}, \mathrm{CHCl}_{3}$ int.): $\delta=1.41-1.49$ ppm (m, 3H), 1.49-1.68 (m, 6H), 1.68-1.90 (m, 8H), 1.92-2.06 (m, 3H), 2.28 (m, $1 \mathrm{H}), 3.74$ (dd, J = 8, $7 \mathrm{~Hz}, 1 \mathrm{H}$ ).

${ }^{13}$ C-NMR (Abb. 92) (150.8 MHz, $\mathrm{CDCl}_{3}, \mathrm{CDCl}_{3}$ int.): $\delta=15.49$ ppm (t), $24.16(\mathrm{t}), 24.80(\mathrm{t})$, $26.05(\mathrm{t}), 27.13(\mathrm{t}), 30.11(\mathrm{t}), 30.50(\mathrm{t}), 32.52(\mathrm{t}), 35.88(\mathrm{t}), 38.01(\mathrm{t}), 44.91(\mathrm{~s}), 48.43(\mathrm{~s})$, 57.55 (s), 72.08 (d).

\section{$(5 R, 11 S)-(+)-T r i s p i r o[3.0 .0 .4 .2 .2]$ tetradecan-11-ol [(5R,11S)-(+)-29)]}

IR (Abb. 15) (KBr): 3100-3555 $\mathrm{cm}^{-1}\left(\mathrm{OH}_{\mathrm{ass}}\right)$.

${ }^{1} \mathbf{H}-\mathbf{N M R}$ (Abb. 52) (600 MHz, $\mathrm{CDCl}_{3}, \mathrm{CHCl}_{3}$ int.): $\delta=1.40-1.46 \mathrm{ppm}(\mathrm{m}, 2 \mathrm{H}), 1.47-1.59$ (m, 6H), 1.59-1.65 (m, 2H), 1.65-1.72 (m, 1H), 1.74-1.88 (m, 6H), $2.07\left(\mathrm{~m}_{\mathrm{c}}, 1 \mathrm{H}\right), 2.18\left(\mathrm{~m}_{\mathrm{c}}\right.$, 1H), 2.34 (dd, J = 9, $7.5 \mathrm{~Hz}, 1 \mathrm{H}), 3.85$ (dd, J = 7.5 Hz, 7.5 Hz, 1H).

${ }^{13}$ C-NMR (Abb. 93)(150.8 MHz, $\mathrm{CDCl}_{3}, \mathrm{CDCl}_{3}$ int.): $\delta=16.01 \mathrm{ppm}(\mathrm{t}), 24.39(\mathrm{t}), 25.09(\mathrm{t})$, $25.33(\mathrm{t}), 28.10(\mathrm{t}), 31.97(\mathrm{t}), 32.23(\mathrm{t}), 32.55(\mathrm{t}), 34.31(\mathrm{t}), 37.68(\mathrm{t}), 46.79(\mathrm{t}), 49.25(\mathrm{~s})$, 57.79 (s), 72.84 (d). 
(1S)-(+)-4,7,7-Trimethyl-3-oxo-2-oxa-bicyclo[2.2.1]heptan-1-carbonsäure(5'S,11'S)trispiro[3.0.0.4.2.2] tetradecan-11-yl-ester [(1S,5' $\left.\left.S, 11^{\prime} S\right)-(+)-79\right]$

Zu einer Lösung eines 96:4-Gemisches aus (5S,11S)-(+)-28 und (5R,11S)-(+)-29 (25 mg, 0.12 mmol) in Pyridin (0.2 ml) gab man bei Raumtemperatur unter Argon und Rühren eine Lösung von (1S)-(-)-4,7,7-Trimethyl-3-oxo-2-oxa-bicyclo[2.2.1] heptan-1-carbonsäurechlorid [(1S)-()-Camphansäurechlorid] (33 mg, $0.14 \mathrm{mmol}$ ) in Pyridin (0.2 ml). Nach 2 h wurde mit Wasser $(8 \mathrm{ml})$ verdünnt und mit Dichlormethan $(3 \times 3 \mathrm{ml})$ extrahiert. Die Extrakte wurden mit $2 \mathrm{~N}$ Schwefelsäure $(4 \mathrm{ml})$ und gesättigter Natriumhydrogencarbonatlösung $(4 \mathrm{ml})$ gewaschen und getrocknet $\left(\mathrm{MgSO}_{4}\right)$. Das Lösungsmittel wurden abgezogen (Badtemperatur $40^{\circ} \mathrm{C} / 15$ Torr) und der feste Rückstand (42 mg, Schmp. 100-105 ${ }^{\circ} \mathrm{C}$ ) aus Ethanol (1.0 ml) durch Diffusion mit Wasser (0.6 ml) kristallisiert. Man erhielt $32 \mathrm{mg}$ (69\%) reines (1S,5'S,11's)-(+)-79 als farblose Kristalle vom Schmp. $109-110^{\circ} \mathrm{C}\left([\alpha]_{\mathrm{D}}{ }^{20}=+29.5\right.$, c 1.28 , Aceton).

$\mathrm{C}_{24} \mathrm{H}_{34} \mathbf{O}_{4}(386.5)$

HRMS $m / e\left(\mathrm{M}^{+}\right) \quad$ Ber.: $386.2457 \quad$ Gef.: 386.2457

IR (Abb. 40) (KBr): 1795,1735 $\mathrm{cm}^{-1}(\mathrm{C}=\mathrm{O})$.

${ }^{1} \mathbf{H}-N M R$ (Abb. 80) (600 MHz, $\mathrm{C}_{6} \mathrm{D}_{6}, \mathrm{C}_{6} \mathrm{D}_{5} \mathrm{H}$ int): $\delta=0.70 \mathrm{ppm}(\mathrm{s}, 3 \mathrm{H}), 0.85$ (s, 3H), 0.87 (s, $3 \mathrm{H}), 1.23\left(\mathrm{~m}_{\mathrm{c}}, 2 \mathrm{H}\right), 1.36\left(\mathrm{~m}_{\mathrm{c}}, 1 \mathrm{H}\right), 1.48-1.56(\mathrm{~m}, 4 \mathrm{H}), 1.58-1.74(\mathrm{~m}, 7 \mathrm{H}), 1.75-1.84(\mathrm{~m}, 2 \mathrm{H})$, $1.88\left(\mathrm{~m}_{\mathrm{c}} 1 \mathrm{H}\right), 1.98-2.21(\mathrm{~m}, 5 \mathrm{H}), 2.25\left(\mathrm{~m}_{\mathrm{c}}, 1 \mathrm{H}\right), 4.83(\mathrm{dd}, \mathrm{J}=8,7.5 \mathrm{~Hz}, 1 \mathrm{H})$.

${ }^{13}$ C-NMR (Abb. 122) (150.8 MHz, $\mathrm{C}_{6} \mathrm{D}_{6}, \mathrm{C}_{6} \mathrm{D}_{6}$ int.): $\delta=9.80 \mathrm{ppm}(\mathrm{q}), 15.85$ (t), 16.51 (q), $16.80(\mathrm{q}), 24.88(\mathrm{t}), 26.08(\mathrm{t}), 26.13(\mathrm{t}), 27.15(\mathrm{t}), 28.78(\mathrm{t}), 30.36(\mathrm{t}), 30.76(\mathrm{t}), 30.99(\mathrm{t})$, $32.68(\mathrm{t}), 35.40(\mathrm{t}), 35.80(\mathrm{t}), 46.10(\mathrm{~s}), 48.76(\mathrm{~s}), 53.79(\mathrm{~s}), 54.62$ (s), 57.63 (s), 74.80 (d), 90.70 (s), 167.37 (s), $177.32(\mathrm{~s})$.

MS (EI): $m / e=134.2(100), 386\left(1, \mathrm{M}^{+}\right)$.

\section{(5R)-(-)-Trispiro[3.0.0.4.2.2]tetradecan [(5R)-(-)-13)]}

(5R)-(-)-24 (111 mg, $0.54 \mathrm{mmol}$ ) wurde wie für das Racemat beschrieben deoxygeniert und lieferte 69 mg (67\%) rohes (5R)-13 (Reinheit 96\% GC). Präparative GC [Säule A, $180^{\circ} \mathrm{C}$, Retentionszeit (min): 3.69] ergab analytisch reines (5R)-(-)-13 als farblose Flüssigkeit (Reinheit $>99.5 \%$ GC). Nach Kapillargaschromatographie an einer chiralen Phase [Säule D, $90^{\circ} \mathrm{C}$; Retentionszeit (min): 33.93] war das Material enantiomerenrein (>99\% ee; $[\alpha]_{\mathrm{D}}{ }^{20}=-4.1$, c 1.19, $\mathrm{CHCl}_{3}$ ). Die ${ }^{1} \mathrm{H}$ - und ${ }^{13} \mathrm{C}-\mathrm{NMR}$-Daten waren mit denen des Racemats identisch.

\section{(5S)-(-)-Trispiro[3.0.0.4.3.2]pentadecan [(5S)-(-)-14]}

(5R)-(-)-24 (204 mg, $1.00 \mathrm{mmol}$ ) wurde wie für das Racemat beschrieben ringerweitert und lieferte 186 mg (85\%) eines 55:45-Gemisches von (5R)-77 und (5R)-78 (Reinheit 93\% GC). 
Dieses Gemisch wurde wie für das Gemisch der Racemate beschrieben deoxygeniert und lieferte 103 mg (59\%) rohes (5S)-14 (Reinheit 84\% GC). Präparative GC [Säule A, $180^{\circ} \mathrm{C}$; Retentionszeit (min): 6.21] ergab analytisch reines (5S)-(-)-14 als farblose Flüssigkeit (Reinheit > 99.5\% GC). Als von (5R)-(-)-24 abgeleitet war (5S)-(-)-14 ebenfalls enantiomerenrein $\left(>99 \%\right.$ ee; $[\alpha]_{\mathrm{D}}{ }^{20}=-0.9$, c $1.22, \mathrm{CHCl}_{3}$ ). Die ${ }^{1} \mathrm{H}$ - und ${ }^{13} \mathrm{C}-\mathrm{NMR}-\mathrm{Daten}$ waren mit denen des Racemats identisch.

\section{Cyclobutyliden-spiro[4.4]nonan (85)}

$\mathrm{Zu}$ einer Suspension von 4-Brombutyltriphenylphosphoniumbromid (28.7 g, $60 \mathrm{mmol}$ ) in trockenem Benzol (80 ml) gab man unter Argon und Rühren in Abständen von 15 min portionsweise Kalium-tert-butylat $(3 \times 4.47 \mathrm{~g}, 120 \mathrm{mmol})$ und erhitzte anschließend auf $50^{\circ} \mathrm{C}$. Nach 3 h tropfte man 71 (2.72 g, $20 \mathrm{mmol})$ zu und erhöhte die Temperatur auf $70^{\circ} \mathrm{C}$. Laut GC [Säule A, $160^{\circ} \mathrm{C}$; Retentionszeiten (min): 5.75 (71), 6.83 (85)] war nach 48 h keine Umsatzänderung mehr zu beobachten. Das Gemisch wurde mit gesättigter Ammoniumchloridlösung ( $3 \mathrm{ml}$ ) hydrolysiert, die überstehende Lösung abdekantiert, der Rückstand mit Pentan $(3 \times 30 \mathrm{ml})$ extrahiert und die vereinigten organischen Phasen über eine $30 \mathrm{~cm}$ Vigreux-Kolonne eingeengt (Badtemperatur bis $120^{\circ} \mathrm{C}$ ). Ausgefallenes Triphenylphosphinoxid wurde abfiltriert und das Filtrat mit Wasser $(6 \times 50 \mathrm{ml})$ gewaschen und getrocknet $\left(\mathrm{MgSO}_{4}\right)$. Die Lösungsmittel wurden abdestilliert und der Rückstand (1.65 g) wurde an Kieselgel in Pentan chromatographiert [Säule $\left.50 \times 2.5, \mathrm{R}_{\mathrm{f}}=0.69(\mathbf{8 5})\right]$. Man erhielt so $288 \mathrm{mg}$ (4\%) rohes 85 (Reinheit 48\% GC). Eine analytisch reine Probe wurde durch präparative GC erhalten. Farblose Flüssigkeit.

$\mathbf{C}_{13} \mathbf{H}_{20}$ (176.3)

HRMS m/e (M+) Ber.: 176.1565 Gef.: 176.1565

IR (Abb. 41) (Film)

${ }^{1}$ H-NMR (Abb. 81) (600 MHz, $\mathrm{CDCl}_{3}, \mathrm{CHCl}_{3}$ int): $\delta=1.38-1.43$ ppm (m, 2H), 1.46-1.49 (m, $2 \mathrm{H}), 1.50-1.58(\mathrm{~m}, 4 \mathrm{H}), 1.59-1.67(\mathrm{~m}, 2 \mathrm{H}), 1.71-1.77(\mathrm{~m}, 2 \mathrm{H}), 1.95\left(\mathrm{~m}_{\mathrm{c}}, 2 \mathrm{H}\right), 2.08\left(\mathrm{~m}_{\mathrm{c}}, 2 \mathrm{H}\right)$, $2.58\left(\mathrm{~m}_{\mathrm{c}}, 2 \mathrm{H}\right), 2.73\left(\mathrm{~m}_{\mathrm{c}}, 2 \mathrm{H}\right)$.

${ }^{13}$ C-NMR (Abb. 123) (150.8 MHz, $\mathrm{CDCl}_{3}, \mathrm{CDCl}_{3}$ int): $\delta=17.75$ ppm (t), $23.69(\mathrm{t}), 25.21(\mathrm{t})$, $29.48(\mathrm{t}), 30.61(\mathrm{t}), 31.38(\mathrm{t}), 37.87(\mathrm{t}), 42.51(\mathrm{t}), 52.73(\mathrm{~s}), 128.94(\mathrm{~s}), 140.03(\mathrm{~s})$.

MS (EI): $m / e=176\left(54, \mathrm{M}^{+}\right), 148$ (100).

\section{5-(4-Nitrobenzolsulfonimido)spiro[3.5]nonan (87)}

Eine Lösung von 4-Nitrobenzolsulfonsäureazid ${ }^{75}$ (311 g, 1.36 mmol) und 69 (140g, 1.14 mol, Reinheit 90\%) in wasserfreiem Acetonitril (1.40 l) wurde unter Argon, Rühren und Licht- 
ausschuss zum Sieden erhitzt. Nach begleitender DC-Analyse $\left[\mathrm{CH}_{2} \mathrm{Cl}_{2}, \mathrm{R}_{\mathrm{f}}=0.88\right.$ (69), 0.64 (87)] war die Reaktion nach 20 h beendet. Die Mischung wurde gekühlt und 24 h bei $-18^{\circ} \mathrm{C}$ kristallisieren lassen. Man erhielt 188 g (57\%) 87 als gelbliche Kristalle vom Schmp. 127$129^{\circ} \mathrm{C}$ (Lit. ${ }^{26} 131^{\circ} \mathrm{C}$ ). Das Filtrat wurde eingeengt, der Rückstand (204 g) in Acetonitril (300 $\mathrm{ml})$ gelöst und $72 \mathrm{~h}$ bei $-18^{\circ} \mathrm{C}$ kristallisieren lassen. Dies lieferte weitere $41 \mathrm{~g}$ (12\%) 87 als gelbliche Kristalle vom Schmp. 133-135 C. Die Gesamtausbeute betrug 229 g (69\%). Die ${ }^{1} \mathrm{H}$ - und ${ }^{13} \mathrm{C}$-NMR-Daten stimmten mit Literaturdaten ${ }^{26}$ überein.

\section{Spiro[3.5]nonan-5-on (32)}

87 (229 g, 0.71 mol) wurde, wie in der Literatur beschrieben, ${ }^{26}$ in einem 1:1-Gemisch von Ethanol und $4 \mathrm{~N}$ wässriger $\mathrm{HCl}$ verseift. Nach DC-Analyse $\left[\mathrm{CH}_{2} \mathrm{Cl}_{2}, \mathrm{R}_{\mathrm{f}}=0.64(87), 0.54\right.$ (32)] war die Reaktion nach $2 \mathrm{~h}$ bei $50^{\circ} \mathrm{C}$ beendet (Lit. $16 \mathrm{~h}$ ). Aufarbeitung und Destillation lieferten 74 g (71\%) 32 als farblose Flüssigkeit vom Sdp. 82-88 ${ }^{\circ} \mathrm{C} / 18$ Torr (Lit.: $78-83^{\circ} \mathrm{C} / 10$ Torr $^{26}$ ). Laut GC [Säule A, $160^{\circ} \mathrm{C}$; Retentionszeiten (min): 6.09 (71), 6.61 (32)] war das Material 98\% rein. Die ${ }^{1} \mathrm{H}$ - und ${ }^{13} \mathrm{C}-\mathrm{NMR}$-Daten stimmten mit Literaturdaten ${ }^{26}$ überein.

\section{Dispiro[3.1.4.3]tridecan-5-on (31)}

Zu einer Suspension von Kaliumhydrid (28.7 g, $251 \mathrm{mmol}$ ) in wasserfreiem Ether (300 ml) tropfte man bei Raumtemperatur unter Argon und Rühren innerhalb von 1.5 h 32 (11.7 g, 85 mmol) zu. Nachdem die Wasserstoffentwicklung beendet war (40 min), wurde 1,4Dibrombutan (21.7 g, $100 \mathrm{mmol}$ ) zugegeben und weiter bei Raumtemperatur gerührt. Nach $24 \mathrm{~h}$ enthielt das Reaktionsgemisch laut GC [Säule A, $180^{\circ} \mathrm{C}$, rel. Retentionszeiten (min): 3.59 (32), 3.82 (1,4-Dibrombutan), 9.61 (31)] noch 35\% 32. Man setzte weiteres 1,4Dibrombutan (6.25 g, $29 \mathrm{mmol}$ ) zu, und nach $90 \mathrm{~h}$ war die Umsetzung beendet. Man verdünnte mit Pentan $(100 \mathrm{ml})$, hydrolysierte mit gesättigter Ammoniumchloridlösung (12 $\mathrm{ml})$, dekantierte $\mathrm{ab}$ und extrahierte den Rückstand mit Pentan $(3 \times 30 \mathrm{ml})$. Die vereinigten organischen Phasen wurden getrocknet $\left(\mathrm{MgSO}_{4}\right)$ und im Rotationsverdampfer eingeengt (Badtemperatur $45^{\circ} \mathrm{C} / 20$ Torr). Destillation des Rückstandes (20.6 g) ergab 12.5 g (76\%) 31 als farblose Flüssigkeit vom Sdp. $104-113^{\circ} \mathrm{C} / 1.3$ Torr.

$$
\mathbf{C}_{13} \mathbf{H}_{20} \mathbf{O} \text { (192.3) }
$$

Ber.: C $81.20 \quad$ H 10.48

Gef.: C $80.88 \quad$ H 10.26

IR (Abb. 16) (Film): $1695 \mathrm{~cm}^{-1}(\mathrm{C}=\mathrm{O})$.

${ }^{1} \mathbf{H}-\mathbf{N M R}$ (Abb. 53) (600 MHz, $\mathrm{CDCl}_{3}, \mathrm{CHCl}_{3}$ int): $\delta=1.28-1.35$ ppm (m, 2H), 1.50-1.62 (m, 6H), 1.62-1.74 (m, 5H), 1.86-1.95 (m, 3H), 1.95-2.02 (m, 2H), 2.43-2.50 (m, 2H). 
${ }^{13}$ C-NMR (Abb. 94) (75.5 MHz, $\mathrm{CDCl}_{3}, \mathrm{CDCl}_{3}$ int): $\delta=15.38 \mathrm{ppm}(\mathrm{t}), 19.28(\mathrm{t}), 24.67(\mathrm{t})$, $29.93(\mathrm{t}), 35.97(\mathrm{t}), 37.62(\mathrm{t}), 38.71(\mathrm{t}), 50.50(\mathrm{~s}), 56.13(\mathrm{~s}), 215.33(\mathrm{~s})$.

MS (EI): $m / e=192\left(5, \mathrm{M}^{+}\right), 41(100)$.

\section{Dispiro[4.0.4.3]tridecan-1-on (30)}

Man erhitzte eine Lösung von 31 (12.5 g, 65 mmol) in Ameisensäure (125 ml) unter Argon und Rühren auf $50-55^{\circ} \mathrm{C}$, bis laut GC [Säule A, $230^{\circ} \mathrm{C}$; Retentionszeiten (min): 5.37 (31), 6.01 (30)] 31 verbraucht war (26 h). Man goss in Eiswasser (750 ml), extrahierte mit Pentan $(5 \times 150 \mathrm{ml})$, wusch die vereinigten Extrakte mit Wasser $(100 \mathrm{ml})$, trocknete über festem Natriumcarbonat und engte im Rotationsverdampfer ein (Badtemperatur $40^{\circ} \mathrm{C} / 20$ Torr). Der Rückstand (11.5 g braunes Öl) wurde an Kieselgel in Pentan/Ether 9:1 chromatographiert [Säule $75 \times 5.5 \mathrm{~cm}, \mathrm{R}_{\mathrm{f}}=0.34$ (30)]. Und lieferte $4.64 \mathrm{~g}$ (38\%) 30 als farblose Flüssigkeit (Reinheit 99\% GC). Die ${ }^{1} \mathrm{H}$ - und ${ }^{13} \mathrm{C}-\mathrm{NMR}$-Daten stimmten mit Literaturdaten ${ }^{23}$ überein.

\section{1-Methylen-dispiro[4.0.4.3]tridecan (33)}

Zu einer Suspension von Methyltriphenylphosphoniumbromid (6.31 g, $17.7 \mathrm{mmol}$ ) in wasserfreiem Ether $(28 \mathrm{ml})$ gab man unter Argon und Rühren Kalium-tert-butylat (1.75 g, 15.6 mmol) und erhitzte zum Sieden. Nach $3 \mathrm{~h}$ wurde alles bis zu einer Badtemperatur von $120^{\circ} \mathrm{C}$ Flüchtige abdestilliert. Anschließend wurde die Temperatur auf $50^{\circ} \mathrm{C}$ gesenkt und $30(1.00 \mathrm{~g}$, $5.20 \mathrm{mmol}$ ) zugegeben. Laut GC [Säule A, $200^{\circ} \mathrm{C}$; Retentionzeiten (min): 3.94 (33), 9.72 (30)] war die Reaktion nach $2.5 \mathrm{~h}$ bei $50^{\circ} \mathrm{C}$ beendet. Man verdünnte mit Pentan (20 ml), hydrolysierte mit Wasser $(0.6 \mathrm{ml})$, dekantierte ab, extrahierte den Rückstand mit Pentan (3 x 8 $\mathrm{ml}$ ) und engte die vereinigten organischen Phasen über eine $20 \mathrm{~cm}$ Vigreux-Kolonne ein (Badtemperatur bis $90^{\circ} \mathrm{C}$ ). Das ausgefallene Triphenylphosphinoxid wurde abgesaugt, das Filtrat nochmals eingeengt und der Rückstand an Kieselgel in Pentan chromatographiert [Säule $30 \times 2.5 \mathrm{~cm}, \mathrm{R}_{\mathrm{f}}=0.72$ (33)]. Man erhielt so $780 \mathrm{mg}$ (81\%) 33 als farblose Flüssigkeit (Reinheit 99\% GC).

$\mathbf{C}_{14} \mathbf{H}_{22}(190.3 \mathrm{~g} / \mathrm{mol})$

Ber.: C $88.35 \quad$ H 11.65

Gef.: C $88.62 \quad$ H 11.31

IR (Abb. 17) (Film): $1645 \mathrm{~cm}^{-1}(\mathrm{C}=\mathrm{C})$.

${ }^{1} \mathbf{H}$-NMR (Abb. 54) (600 MHz, $\mathrm{C}_{6} \mathrm{D}_{6}, \mathrm{C}_{6} \mathrm{D}_{5} \mathrm{H}$ int): $\delta=1.19-1.25$ ppm (m, 1H), 1.30-1.39 (m, 2H), 1.39-1.47 (m, 2H), 1.48-1.66 (m, 9H), 1.66-1.74 (m, 3H), 1.80-1.86 (m, 1H), 2.22-2.30 (m, 1H), 2.30-2.37 (m, 1H), 4.80 (ddd, $\mathrm{J}=2.5,1.25,1.25 \mathrm{~Hz}, 1 \mathrm{H}$ ), 5.04 (ddd, $\mathrm{J}=$ 2.5, 1.25, $1.25 \mathrm{~Hz}, 1 \mathrm{H})$. 
${ }^{13}$ C-NMR (Abb. 95) (150.8 MHz, $\mathrm{C}_{6} \mathrm{D}_{6}, \mathrm{C}_{6} \mathrm{D}_{6}$ int.): $\delta=20.66 \mathrm{ppm}(\mathrm{t}), 23.74(\mathrm{t}), 24.54(\mathrm{t})$, $24.64(\mathrm{t}), 33.98(\mathrm{t}), 35.17(\mathrm{t}), 37.00(\mathrm{t}), 37.72(\mathrm{t}), 38.05(\mathrm{t}), 40.13(\mathrm{t}), 56.48(\mathrm{~s}), 57.96(\mathrm{~s})$, $106.54(\mathrm{t}), 158.15(\mathrm{~s})$.

MS (EI): $m / e=190\left(10, \mathrm{M}^{+}\right), 108(100)$.

\section{Dichloressigsäure-2,2-dichlor-1-dispiro[4.0.4.3]tridec-1-en-1-yl-methyl-vinyl-ester (94) und Essigsäure-2,2-dichlor-1-dispiro[4.0.4.3] tridec-1-en-1-yl-methyl-vinyl-ester (95)}

Zu einer Mischung aus Zinkstaub (300 mg, $4.60 \mathrm{mmol}$ ) und 33 (288 mg, $1.52 \mathrm{mmol}$ ) in wasserfreiem Ether $(3.0 \mathrm{ml})$ tropfte man unter Argon und Ultraschall innerhalb von $10 \mathrm{~min}$ eine Lösung von Trichloracetylchlorid (602 mg, $3.30 \mathrm{mmol}$ ) in Ether (3.0 ml) und erhitzte anschließend zum Sieden. Nach 6 h war nach GC-Analyse [Säule B, 2 min $200^{\circ} \mathrm{C}, 30^{\circ} \mathrm{C} / \mathrm{min}$ auf $230^{\circ} \mathrm{C}$; Retentionszeiten (min): 1.68 (33), 13.09 (94)] keine Umsatzänderung mehr zu beobachten. Man filtrierte über Celite, wusch den Rückstand mit Ether $(3 \times 8 \mathrm{ml})$ und die vereinigten organischen Phasen mit Wasser $(20 \mathrm{ml})$, gesättigter Natriumhydrogencarbonat(20 ml) und gesättigter Natriumchloridlösung $(20 \mathrm{ml})$. Nach Trocknen $\left(\mathrm{MgSO}_{4}\right)$ wurde das Lösungsmittel im Rotationsverdampfer abgezogen (Badtemperatur $40^{\circ} \mathrm{C} / 15$ Torr). Der Rückstand (560 mg) bestand laut GC-Analyse aus einem 2:1-Gemisch von 94 und nicht umgesetztem 33. Zur Aufnahme der Spektren wurde 94 (Reinheit 90\%) präparativ gaschromatographisch isoliert.

Das verbliebene Material (500 mg) wurde zu einer Suspension von Zinkstaub (785 mg, 12 mmol) in konzentrierter Essigsäure $(2.0 \mathrm{ml})$ gegeben und unter Argon und Rühren auf $60^{\circ} \mathrm{C}$ erhitzt. Nach einer Stunde war die Reaktion nach GC-Analyse beendet [Säule B, $230^{\circ} \mathrm{C}$; Retentionszeiten (min): 6.82 (94), 10.69 (95)]. Man filtrierte über Celite, wusch den Rückstand mit Pentan $(3 \times 8 \mathrm{ml})$ und versetzte anschließend mit Wasser $(20 \mathrm{ml})$. Man trennte die Phasen, extrahierte die wässrige mit Pentan $(3 \times 15 \mathrm{ml})$, wusch die vereinigten organischen Phasen mit $1 \mathrm{~N} \mathrm{NaOH}$ (50 ml) und gesättigter Natriumchloridlösung (50 ml), und trocknete $\left(\mathrm{MgSO}_{4}\right)$. Man zog das Lösungsmittel im Rotationsverdampfer ab (Badtemperatur $40^{\circ} \mathrm{C} / 15$ Torr) und chromatographierte den Rückstand (320 mg) an Kieselgel in Pentan/Ether 95:5 [Säule $45 \times 2.5 \mathrm{~cm}, \mathrm{R}_{\mathrm{f}}=0.79$ (33), 0.38 (95)]. Man erhielt so $64 \mathrm{mg}$ (22\%) unumgesetztes 33 und $87 \mathrm{mg}$ (17\%) 95 als farblose Flüssigkeiten.

\section{Dichloressigsäure-2,2-dichlor-1-dispiro[4.0.4.3]tridec-1-en-1-yl-methyl-vinyl-ester (94)}

${ }^{1} \mathbf{H}-\mathbf{N M R}$ (Abb. 82) (600 MHz, $\mathrm{CDCl}_{3}, \mathrm{CHCl}_{3}$ int): $\delta=1.26-1.33$ ppm (m, 3H), 1.42-1.60 (m, 9H), 1.60-1.68 (m, 2H), 1.85 (ddd, $\mathrm{J}=12.5,8.5,8.5 \mathrm{~Hz}, 1 \mathrm{H}$ ), 2.02 (ddd, $\mathrm{J}=12.5,8,4 \mathrm{~Hz}$, 1H), 2.10-2.20 (m, 2H), 3.24 (dddd, $\mathrm{J}=17,2,2,2 \mathrm{~Hz}, 1 \mathrm{H}$ ), 3.29 (dddd, $\mathrm{J}=17,2$, 2, $2 \mathrm{~Hz}$, 1H), 5.59 (dddd, $\mathrm{J}=$ 2, 2, 2, $2 \mathrm{~Hz}, 1 \mathrm{H}$ ), 6.00 (s, 1H). 
${ }^{13}$ C-NMR (Abb. 124) (150.8 MHz, $\mathrm{CDCl}_{3}, \mathrm{CDCl}_{3}$ int): $\delta=20.99$ ppm (t), $23.76(\mathrm{t}), 24.15$ (t), $30.06(\mathrm{t}), 31.84(\mathrm{t}), 33.98(\mathrm{t}), 34.73(\mathrm{t}), 37.17(\mathrm{t}), 37.29(\mathrm{t}), 37.62(\mathrm{t}), 57.36(\mathrm{~s}), 62.17(\mathrm{~s})$, 63.51 (d), 116.32 (s), 129.85 (d), 140.81 (s), 145.21 (s), 160.78 (s).

Essigsäure-2,2-dichlor-1-dispiro[4.0.4.3]tridec-1-en-1-yl-methyl-vinyl-ester (95)

$\mathrm{C}_{18} \mathrm{H}_{24} \mathrm{Cl}_{2} \mathrm{O}_{2}$ (343.3)

Ber.: C $62.98 \quad$ H 7.05

Gef.: C $62.70 \quad$ H 7.12

IR (Abb. 42) (Film): $1775 \mathrm{~cm}^{-1}(\mathrm{C}=\mathrm{O})$.

${ }^{1} \mathbf{H}-\mathbf{N M R}$ (Abb. 83) (600 MHz, $\mathrm{CDCl}_{3}, \mathrm{CHCl}_{3}$ int): $\delta=1.26-1.35$ ppm (m, 3H), 1.43-1.67 (m, 11H), 1.85 (ddd, J = 12, 8, 8 Hz, 1H), 2.02 (ddd, J = 12, 8, 4 Hz, 1H), 2.06-2.20 (m, 2H), 2.15 (s, 3H), 3.14 (dddd, $\mathrm{J}=17,1.5,1.5,1.5 \mathrm{~Hz}, 1 \mathrm{H}$ ), 3.23 (dddd, $\mathrm{J}=17,1.5,1.5,1.5 \mathrm{~Hz}, 1 \mathrm{H}$ ), 5.55 (dddd, $\mathrm{J}=1.5,1.5,1.5,1.5 \mathrm{~Hz}, 1 \mathrm{H}$ ).

${ }^{13}$ C-NMR (Abb. 125) (150.8 MHz, $\mathrm{CDCl}_{3}, \mathrm{CDCl}_{3}$ int): $\delta=20.53$ ppm (q), 21.01 (t), 23.87 (t), $24.26(\mathrm{t}), 30.04(\mathrm{t}), 32.18(\mathrm{t}), 33.86(\mathrm{t}), 34.87(\mathrm{t}), 37.14(\mathrm{t}), 37.41(\mathrm{t}), 37.82(\mathrm{t}), 57.25(\mathrm{~s})$, 62.27 (s), 114.77 (s), 128.73 (d), 141.91 (s), 146.08 (s), 167.38 (s).

MS (EI): $m / e=299\left(2, \mathrm{M}^{+}-\mathrm{CH}_{3} \mathrm{CO}\right), 43.0$ (100).

\section{$\left(1 S^{*}, 5 S^{*}\right)-1-(1-E t h o x y-v i n y l)-d i s p i r o[4.0 .4 .3] t r i d e c a n-1-o l ~\left[\left(1 S^{*}, 5 S^{*}\right)-41\right]$}

Zu einer Lösung von Ethylvinylether (7.22 g, $100 \mathrm{mmol}$ ) in wasserfreiem THF (85 ml) tropfte man bei $-78^{\circ} \mathrm{C}$ unter Argon und Rühren innerhalb von 50 min eine 1.5 molare Lösung von tert-Butyllithium in Hexan (40 ml, $60 \mathrm{mmol}$ ). 15 min nach beendeter Zugabe ließ man auf Raumtemperatur erwärmen und verwendete die resultierende gelbliche Lösung von 1-Ethoxyvinyllithium im nächsten Schritt. Eine Suspension von fein gepulvertem trockenem $\mathrm{CeCl}_{3}$ (9.60 g, $39.0 \mathrm{mmol})$ in wasserfreiem THF (145 ml) wurde $2 \mathrm{~h}$ bei Raumtemperatur unter Argon gerührt und anschließend mit 30 (3.10 g, 16.1 mmol) versetzt. Nach weiteren 2 h bei Raumtemperatur wurde auf $0^{\circ} \mathrm{C}$ gekühlt, die frisch hergestellte 1-Ethoxyvinyllithiumlösung innerhalb von 45 min zugetropft und das Gemisch anschließend bei Raumtemperatur gerührt. Laut GC [Säule B, $210^{\circ} \mathrm{C}$; Retentionszeiten (min): 2.81 (30), 4.32 ((1S*,5S*)-41)] war nach 45 min keine Umsatzänderung mehr zu beobachten. Man verdünnte mit Pentan (100 ml), hydrolysierte mit gesättigter Ammoniumchloridlösung (40 ml) und saugte ab. Man wusch den Rückstand mit Pentan $(4 \times 50 \mathrm{ml})$, und die vereinigten Filtrate mit Wasser $(2 \times 250 \mathrm{ml})$. Die Phasen wurden getrennt, die wässrige mit Pentan $(250 \mathrm{ml})$ extrahiert und die vereinigten organischen Phasen getrocknet $\left(\mathrm{MgSO}_{4}\right)$. Man zog das Lösungsmittel im Rotationsverdampfer ab (Badtemperatur $50^{\circ} \mathrm{C} / 15$ Torr) und erhielt so $3.88 \mathrm{~g}(94 \%)$ rohes $\left(1 S^{*}, 5 S^{*}\right)-41$ als gelbliche Flüssigkeit (Reinheit 81\% GC). Dieses Material wurde als solches weiter umgesetzt. Für die Aufnahme der Spektren wurde eine Probe präparativ gaschromatographisch gereinigt. 
$\mathrm{C}_{17} \mathrm{H}_{28} \mathbf{O}_{2}$ (264.4)

HRMS m/e $\left(\mathrm{M}^{+}\right) \quad$ Ber.: 264.2089 Gef.: 264.2089

IR (Abb. 19) (Film): $3600-3300 \mathrm{~cm}^{-1}\left(\mathrm{OH}_{\mathrm{ass}}\right)$.

${ }^{1} \mathbf{H}-N M R$ (Abb. 56) (600 MHz, $\mathrm{C}_{6} \mathrm{D}_{6}, \mathrm{C}_{6} \mathrm{D}_{5} \mathrm{H}$ int): $\delta=0.97 \mathrm{ppm}(\mathrm{t}, \mathrm{J}=7 \mathrm{~Hz}, 3 \mathrm{H}), 1.39-1.86$ (m, 16H), 1.92-1.99 (m, 1H), 2.13-2.22 (m, 3H), 2.41 (m $\left.\mathrm{m}_{\mathrm{c}}, 1 \mathrm{H}\right), 3.32$ (q, J = $\left.7 \mathrm{~Hz}, 2 \mathrm{H}\right), 3.93$ (d, J = $2 \mathrm{~Hz}, 1 \mathrm{H}), 4.40$ (d, J = $2 \mathrm{~Hz}, 1 \mathrm{H})$.

${ }^{13}$ C-NMR (Abb. 97) (150.8 MHz, $\mathrm{C}_{6} \mathrm{D}_{6}, \mathrm{C}_{6} \mathrm{D}_{6}$ int): $\delta=14.33$ ppm (q), 20.11 (t), 20.34 (t), $24.29(\mathrm{t}), 25.52(\mathrm{t}), 33.64(\mathrm{t}), 35.61(\mathrm{t}), 35.77(\mathrm{t}), 36.45(\mathrm{t}), 39.25(\mathrm{t}), 41.47(\mathrm{t}), 56.62(\mathrm{~s})$, $60.92(\mathrm{~s}), 62.88(\mathrm{t}), 82.14(\mathrm{t}), 85.77(\mathrm{~s}), 166.29(\mathrm{~s})$.

MS (EI): $m / e=264\left(22, \mathrm{M}^{+}\right), 97(100)$.

\section{$\left(1 S^{*}, 5 S^{*}\right)-1-(1-E t h o x y-c y c l o p r o p y l)-d i s p i r o[4.0 .4 .3] t r i d e c a n-1-o l ~\left[\left(1 S^{*}, 5 S^{*}\right)-42\right]$}

Zu einer Suspension von frisch hergestelltem Zink/Silber-Paar ${ }^{67}$ (9.2 g) in wasserfreiem Ether (50 ml) gab man unter Argon und Rühren Diiodmethan (20.7 g, 77.4 mmol), wobei ein exothermer Effekt und selbsttätiger Rückfluß zu beobachten waren. Nach 20 min setzte man (1S*,5S*)-41 (3.65 g, 13.8 mmol, Reinheit 81\%) zu, und weiteren 1.5 h Rückfluß war die

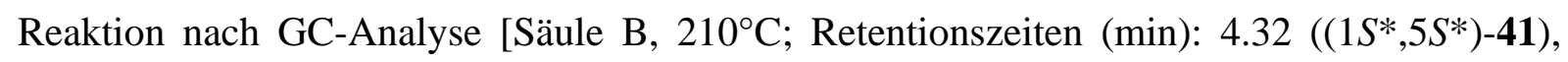
$\left.6.41\left(\left(1 S^{*}, 5 S^{*}\right)-42\right)\right]$ beendet. Man verdünnte mit Pentan $(130 \mathrm{ml})$ und hydrolysierte mit gesättigter Ammoniumchloridlösung (1.6 ml, 15 min Rühren). Die überstehende Lösung wurde abdekantiert und der Rückstand mit Ether $(3 \times 50 \mathrm{ml})$ extrahiert. Die vereinigten organischen Phasen wurden mit gesättigter Ammoniumchloridlösung $(80 \mathrm{ml})$ und Wasser $(2 \times 80$ $\mathrm{ml}$ ) gewaschen, getrocknet $\left(\mathrm{MgSO}_{4}\right)$ und im Rotationsverdampfer (Badtemperatur $50^{\circ} \mathrm{C} / 15$ Torr) eingeengt. Man erhielt so $2.65 \mathrm{~g}(69 \%)$ rohes $\left(1 S^{*}, 5 S^{*}\right)-42$ als gelbliche Flüssigkeit (Reinheit 80\% GC). Dieses Material wurde als solches weiter umgesetzt. Für die Aufnahme der Spektren wurde eine Probe präparativ gaschromatographisch gereinigt.

$\mathrm{C}_{18} \mathrm{H}_{30} \mathrm{O}_{2}$ (278.4)

HRMS $m / e\left(\mathrm{M}^{+}\right) \quad$ Ber.: $278.2246 \quad$ Gef.: 278.2246

IR (Abb. 20) (Film): 3600-3400 $\mathrm{cm}^{-1}\left(\mathrm{OH}_{\mathrm{ass}}\right)$.

${ }^{1} \mathbf{H}-N M R$ (Abb. 57) $\left(600 \mathrm{MHz}, \mathrm{C}_{6} \mathrm{D}_{6}, \mathrm{C}_{6} \mathrm{D}_{5} \mathrm{H}\right.$ int): $\delta=0.62-0.68 \mathrm{ppm}(\mathrm{m}, 1 \mathrm{H}), 0.67-0.76$ (m, $3 \mathrm{H}), 0.98$ (dd, $\mathrm{J}=7,7 \mathrm{~Hz}, 3 \mathrm{H}), 1.20-1.28(\mathrm{~m}, 2 \mathrm{H}), 1.32\left(\mathrm{~m}_{\mathrm{c}}, 1 \mathrm{H}\right), 1.42-1.83(\mathrm{~m}, 13 \mathrm{H}), 1.87$ (ddd, $\mathrm{J}=12,10,5 \mathrm{~Hz}, 1 \mathrm{H}$ ), 2.02-2.10 (m, 2H), 2.18-2.24 (m, 1H), 2.30 (ddd, J = 13, 10, $7 \mathrm{~Hz}$, 1H), $3.13(\mathrm{dq}, \mathrm{J}=9,7 \mathrm{~Hz}, 1 \mathrm{H}), 3.35$ (dq, $\mathrm{J}=9,7 \mathrm{~Hz}, 1 \mathrm{H})$.

${ }^{13}$ C-NMR (Abb. 98) (150.8 MHz, $\mathrm{C}_{6} \mathrm{D}_{6}, \mathrm{C}_{6} \mathrm{D}_{6}$ int): $\delta=8.89 \mathrm{ppm}(\mathrm{t}), 10.37(\mathrm{t}), 16.09(\mathrm{t})$, $20.21(\mathrm{q}), 20.69(\mathrm{t}), 24.25(\mathrm{t}), 25.34(\mathrm{t}), 34.55(\mathrm{t}), 35.62(\mathrm{t}), 36.43(\mathrm{t}), 38.29(\mathrm{t}), 39.14(\mathrm{t})$, $40.33(\mathrm{t}), 56.65(\mathrm{~s}), 61.36(\mathrm{~s}), 63.41(\mathrm{t}), 65.20(\mathrm{~s}), 87.01(\mathrm{~s})$ 
MS (EI): $m / e=278\left(8, \mathrm{M}^{+}\right), 108(100)$.

\section{$\left(4 R^{*}, 5 S^{*}\right)$-Trispiro[3.0.0.4.3.3]hexadecan-1-on [(4R*,5S*)-43]}

Ein Mischung aus (1S*,5S*)-42 (2.42 g, 8.68 mmol, Reinheit 80\%) und $8 \mathrm{M}$ wässriger Tetrafluorborsäure (4.40 ml, $35.2 \mathrm{mmol})$ in Ether $(85 \mathrm{ml})$ wurde bei Raumtemperatur gerührt. Laut ${ }^{1} \mathrm{H}-\mathrm{NMR}$ war die Reaktion nach $2 \mathrm{~h}$ beendet. Die Mischung wurde mit gesättigter Natriumhydrogencarbonatlösung $(3 \times 50 \mathrm{ml})$ behandelt, mit Wasser $(2 \times 50 \mathrm{ml})$ gewaschen und getrocknet $\left(\mathrm{MgSO}_{4}\right)$. Das Lösungsmittel wurde abgezogen (Badtemperatur $45^{\circ} \mathrm{C} / 15$ Torr) und der Rückstand (2.12 g) an Kieselgel in Pentan/Ether 9:1 chromatographiert [Säule $75 \times 5.5$ $\left.\mathrm{cm}, \mathrm{R}_{\mathrm{f}}=0.42\left(\left(4 R^{*}, 5 S^{*}\right)-43\right)\right]$. Man erhielt so $1.09 \mathrm{~g}(64 \%)\left(4 R^{*}, 5 S^{*}\right)-43$ als farblose Flüssigkeit. Laut GC [Säule B, $210^{\circ} \mathrm{C}$; Retentionszeit (min): $5.88\left(\left(4 R^{*}, 5 S^{*}\right)-43\right)$ ] war das Material $94 \%$ rein.

\section{$\mathrm{C}_{16} \mathrm{H}_{24} \mathrm{O}(232.4)$}

Ber.: C $82.70 \quad$ H 10.41

Gef.: C $82.54 \quad$ H 10.56

IR (Abb. 21) (Film): $1770 \mathrm{~cm}^{-1}(\mathrm{C}=\mathrm{O})$.

${ }^{1} \mathbf{H}$-NMR (Abb. 58) (600 MHz, $\mathrm{CDCl}_{3}, \mathrm{CHCl}_{3}$ int): $\delta=1.20 \mathrm{ppm}\left(\mathrm{m}_{\mathrm{c}}, 1 \mathrm{H}\right), 1.27\left(\mathrm{~m}_{\mathrm{c}}, 1 \mathrm{H}\right)$, 1.35-1.80 (m, 16H), 1.86 (ddd, $\mathrm{J}=12.5,9,9 \mathrm{~Hz}, 1 \mathrm{H}), 1.95\left(\mathrm{~m}_{\mathrm{c}}, 1 \mathrm{H}\right), 2.03$ (ddd, $\mathrm{J}=10.5,8,4$ Hz, 1H), 2.25 (ddd, J = 10.5, 10, 7 Hz, 1H), 2.77 (ddd, J = 18, 10.5, 5.5 Hz, 1H), 2.88 (ddd, J $=18,10,7 \mathrm{~Hz}, 1 \mathrm{H})$.

${ }^{13}$ C-NMR (Abb. 99) (150.8 MHz, $\mathrm{CDCl}_{3}, \mathrm{CDCl}_{3}$ int): $\delta=19.92 \mathrm{ppm}(\mathrm{t}), 20.65(\mathrm{t}), 21.36(\mathrm{t})$, $22.71(t), 23.81(t), 34.71(t), 34.77(t), 35.39(t), 35.59(t), 38.79(t), 38.85(t), 40.43(t), 55.91$ (s), 58.95 (s), 76.10 (s), 216.42 (s).

MS (EI): $m / e=232\left(23, \mathrm{M}^{+}\right), 108(100)$.

\section{$\left(4 R^{*}, 5 S^{*}\right)-\mathrm{N}$-Trispiro[3.0.0.4.3.3] $\left[\left(4 R^{*}, 5 S^{*}\right)-98\right]$}

$\mathrm{Zu}$ einer Lösung von 2,4-Dinitrophenylhydrazin (100 $\mathrm{mg}, 0.50 \mathrm{mmol}$ ) in konzentrierter Schwefelsäure $(0.50 \mathrm{ml})$, Wasser $(0.75 \mathrm{ml})$ und Ethanol $(2.5 \mathrm{ml})$ gab man $\left(4 R^{*}, 5 S^{*}\right)-43$ (50 mg, $0.22 \mathrm{mmol}$ ). Man rührte $15 \mathrm{~min}$ und ließ die zunächst klare Lösung sieben Tage bei Raumtemperatur stehen. Die ausgefallenen orangen Kristalle wurden abfiltriert, mit Wasser $(5 \times 1 \mathrm{ml})$ und Aceton $(0.5 \mathrm{ml})$ gewaschen und getrocknet. Ausbeute: $58 \mathrm{mg}$ (63\%). Schmp. $152^{\circ} \mathrm{C}$.

$\mathrm{C}_{22} \mathrm{H}_{28} \mathrm{~N}_{4} \mathrm{O}_{4}(412.5)$ 
Ber.: C $64.06 \quad$ H 6.84

Gef.: C 64.32 H 6.64

IR (Abb. 43) (KBr): $3330(\mathrm{~N}-\mathrm{H}), 1625 \mathrm{~cm}^{-1}(\mathrm{C}=\mathrm{N})$.

${ }^{1} \mathbf{H}$-NMR (Abb. 84) (600 MHz, $\mathrm{CDCl}_{3}, \mathrm{CHCl}_{3}$ int): $\delta=1.24-1.36$ ppm (m, 3H), 1.39-1.74 (m, $12 \mathrm{H}), 1.76-1.90$ (m, 3H), 2.02-2.13 (m, 3H), 2.41 (ddd, $\mathrm{J}=12,10,7 \mathrm{~Hz}, 1 \mathrm{H}), 2.77-2.89$ (m 2H), 7.78 (d, J = 9.5 Hz, 1H), 8.25 (dd, J = 9.5, $2.5 \mathrm{~Hz}, 1 \mathrm{H}$ ), 9.08 (d, J = 2.5 Hz, 1H), 10.67 (br s, 1H).

${ }^{13}$ C-NMR (Abb. 126) (150.8 MHz, $\mathrm{CDCl}_{3}, \mathrm{CDCl}_{3}$ int): $\delta=19.74$ ppm (t), 20.33 (t), $22.99(\mathrm{t})$, $24.09(\mathrm{t}), 24.19(\mathrm{t}), 25.70(\mathrm{t}), 34.36(\mathrm{t}), 34.99(\mathrm{t}), 35.06(\mathrm{t}), 35.71(\mathrm{t}), 39.58(\mathrm{t}), 41.01(\mathrm{t}), 56.09$ (s), 58.50 (s), 63.48 (s), 116.03 (d), 123.63 (d), 128.65 (s), 130.05 (d), 137.35 (s), 144.91 (s), 169.40 (s).

MS (EI): $m / e=412\left(17, \mathrm{M}^{+}\right), 263(100)$.

\section{$\left(5 S^{*}\right)$-Trispiro[3.0.0.4.3.3]hexadecan [(5S*)-15]}

Zu einer Lösung von Natrium (276 mg, $12.0 \mathrm{mmol}$ ) in Diethylenglykol (16 ml) gab man unter Argon und Rühren (4R*,5S*)-43 (232 mg, $1.0 \mathrm{mmol})$ und wasserfreies Hydrazin (2.60 g, 81 mmol) und erhitzte auf $180^{\circ} \mathrm{C}$. Nach GC-Analyse [Säule A, $230^{\circ} \mathrm{C}$; Retentionszeiten (min):

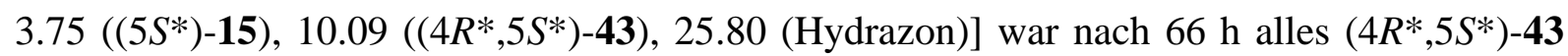
verbraucht. Das Reaktionswasser und überschüssiges Hydrazin wurden unter strömendem Argon weitestgehend abdestilliert, wobei die Temperatur schrittweise auf $200-205^{\circ} \mathrm{C}$ erhöht wurde. Nach 72 h bei dieser Temperatur war die Reduktion laut GC komplett. Man spülte die Apparatur mit Pentan (20 ml), goß das Reaktionsgemisch in Wasser $(60 \mathrm{ml})$ und extrahierte mit Pentan $(2 \times 60 \mathrm{ml})$. Man wusch die vereinigten organischen Phasen mit Wasser $(3 \times 60$ $\mathrm{ml}$ ), trocknete $\left(\mathrm{MgSO}_{4}\right)$, zog das Lösungsmittel ab (Badtemperatur $35^{\circ} \mathrm{C} / 15$ Torr), und unterwarf den Rückstand einer Kurzweg-Chromatographie an Kieselgel in Pentan (Säule $15 \times 1$ $\mathrm{cm}, \mathrm{R}_{\mathrm{f}}=0.77$ ). Nach Verdampfen des Lösungsmittels (Badtemperatur $50^{\circ} \mathrm{C} / 15$ Torr) verblieben $140 \mathrm{mg}$ (58\%) rohes (5S*)-15 als farblose Flüssigkeit (Reinheit 90\% GC). Eine analytisch reine Probe wurde über präparative GC erhalten.

$\mathbf{C}_{16} \mathbf{H}_{26}$ (218.4)

Ber.: C $88.00 \quad$ H 12.00

Gef.: C $87.87 \quad$ H 11.83

IR (Abb. 11) (Film)

${ }^{1} \mathbf{H}-\mathbf{N M R}$ (Abb. 48) (600 MHz, $\mathrm{CDCl}_{3}, \mathrm{CHCl}_{3}$ int): $\delta=1.24-1.29$ ppm (m, 1H), 1.30-1.38 (m, 4H), 1.43-1.74 (m, 16H), 1.78-1.90 (m, 2H), 1.93 (ddd, J = 12, 8, 4 Hz, 1H), 2.25 (ddd, J = 10, 10, $10 \mathrm{~Hz}, 1 \mathrm{H}$ ), 2.31 (ddd, $\mathrm{J}=10,10,10 \mathrm{~Hz}, 1 \mathrm{H}$ ). 
${ }^{13}$ C-NMR (Abb. 89) (150.8 MHz, $\mathrm{CDCl}_{3}, \mathrm{CDCl}_{3}$ int): $\delta=15.91 \mathrm{ppm}(\mathrm{t}), 19.58(\mathrm{t}), 20.59(\mathrm{t})$, $23.70(t), 24.72(t), 30.25(t), 31.95(t), 32.55(t), 34.12(t), 35.14(t), 35.81(t), 40.38(t), 41.39$ (t), $52.08(\mathrm{~s}), 55.73(\mathrm{~s}), 56.49(\mathrm{~s})$.

MS (EI): $m / e=218\left(1, \mathrm{M}^{+}\right), 79(100)$.

\section{$\left(4 R^{*}, 5 S^{*}\right)-1-M e t h y l e n-t r i s p i r o[3.0 .0 .4 .3 .3]$ hexadecan $\left[\left(4 R^{*}, 5 S^{*}\right)-44\right]$}

Zu einer Suspension von Methyltriphenylphosphoniumbromid (3.97 g, $11.1 \mathrm{mmol}$ ) in trockenem Benzol (20 ml) gab man unter Argon und Rühren Kalium-tert-butylat (1.12 g, 10.0 mmol) und erhitzte zum Sieden. Nach 2.5 h destillierte man alles bis zu einer Badtemperatur von $120^{\circ} \mathrm{C}$ Flüchtige ab und versetzte anschließend tropfenweise mit (4R*,5S*)-43 (700 mg, $2.92 \mathrm{mmol})$. Laut GC [Säule B, $200^{\circ} \mathrm{C}$; Retentionszeiten (min): 3.73 ((4R*,5S*)-44), 7.56 $\left.\left(\left(4 R^{*}, 5 S^{*}\right)-43\right)\right]$ war die Umsetzung nach $1.5 \mathrm{~h}$ bei $120^{\circ} \mathrm{C}$ komplett. Man verdünnte mit Pentan $(40 \mathrm{ml})$, hydrolysierte mit Wasser $(0.8 \mathrm{ml})$, dekantierte ab, extrahierte den Rückstand mit Pentan $(3 \times 8 \mathrm{ml})$ und engte die vereinigten organischen Phasen über eine $30 \mathrm{~cm}$ VigreuxKolonne ein (Badtemperatur bis $90^{\circ} \mathrm{C}$ ). Das ausgefallene Triphenylphosphinoxid wurde abfiltriert und das Filtrat eingeengt und an Kieselgel in Pentan chromatographiert [Säule $30 \times 2.5$ $\left.\mathrm{cm}, \mathrm{R}_{\mathrm{f}}=0.66\left(\left(4 R^{*}, 5 S^{*}\right)-44\right)\right]$. Man erhielt so $582 \mathrm{mg}(87 \%)\left(4 R^{*}, 5 S^{*}\right)-44$ als farblosen glasartigen Feststoff vom Schmp. $27-33^{\circ} \mathrm{C}$ (Reinheit $>99 \%$ GC).

$\mathrm{C}_{17} \mathrm{H}_{26}$ (230.4)

Ber.: C $88.63 \quad$ H 11.37

Gef.: C $88.53 \quad$ H 11.09

IR (Abb. 22) (Film): $1660 \mathrm{~cm}^{-1}(\mathrm{C}=\mathrm{C})$.

${ }^{1} \mathbf{H}-\mathbf{N M R}$ (Abb. 59) (600 MHz, $\mathrm{C}_{6} \mathrm{D}_{6}, \mathrm{C}_{6} \mathrm{D}_{5} \mathrm{H}$ int): $\delta=1.30-1.42 \mathrm{ppm}$ (m, 3H), 1.43-1.48 (m, $1 \mathrm{H}), 1.50-1.80(\mathrm{~m}, 14 \mathrm{H}), 1.87\left(\mathrm{~m}_{\mathrm{c}}, 1 \mathrm{H}\right), 1.96-2.01(\mathrm{~m}, 1 \mathrm{H}), 2.15-2.26(\mathrm{~m}, 2 \mathrm{H}), 2.46-2.56$ (m, 2H), 4.83 (dd, J = 2.25, $2.25 \mathrm{~Hz}, 1 \mathrm{H}$ ), 4.85 (dd, J = 2.25, $2.25 \mathrm{~Hz}, 1 \mathrm{H}$ ).

${ }^{13}$ C-NMR (Abb. 100) (150.8 MHz, $\mathrm{C}_{6} \mathrm{D}_{6}, \mathrm{C}_{6} \mathrm{D}_{6}$ int): $\delta=19.58 \mathrm{ppm}(\mathrm{t}), 19.79(\mathrm{t}), 23.18(\mathrm{t})$, $24.07(t), 25.53(t), 26.18(t), 33.23(t), 34.89(t), 35.18(t), 36.01(t), 39.57(t), 43.66(t), 56.40$ (s), $57.33(\mathrm{~s}), 60.21(\mathrm{~s}), 106.24(\mathrm{t}), 158.57(\mathrm{~s})$.

MS (EI): $m / e=230\left(28, \mathrm{M}^{+}\right), 121(100)$.

\section{$\left(3 S^{*}, 4 R^{*}, 5 S^{*}\right)-1-O x a-t e t r a s p i r o[2.0 .0 .0 .4 .3 .3 .2] o c t a d e c a n ~\left[\left(3 S^{*}, 4 R^{*}, 5 S^{*}\right)-46\right]$}

Zu einer Lösung von (4R*,5S*)-44 (582 mg, $2.53 \mathrm{mmol})$ in Dichlormethan (8.0 ml) gab man 3-Chlorperbenzoesäure (940 mg, 70\% w/w, $3.80 \mathrm{mmol}$ ) und rührte das Gemisch bei Raum-

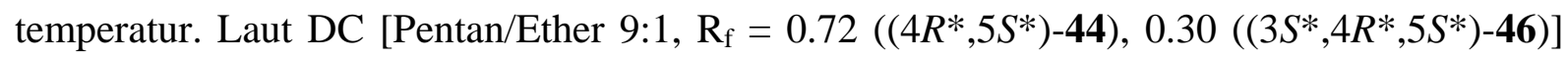
war die Reaktion nach 1.5 h beendet. Man wusch mit 1 N KOH (6 ml), extrahierte die wäss- 
rige Phase mit Dichlormethan $(3 \times 8 \mathrm{ml})$, wusch die vereinigten organischen Phasen mit Wasser $(2 \times 15 \mathrm{ml})$ und trocknete $\left(\mathrm{MgSO}_{4}\right)$. Man engte im Rotationsverdampfer ein (Badtemperatur $40^{\circ} \mathrm{C} / 15$ Torr) und chromatographierte den festen Rückstand (650 mg) an Kieselgel in

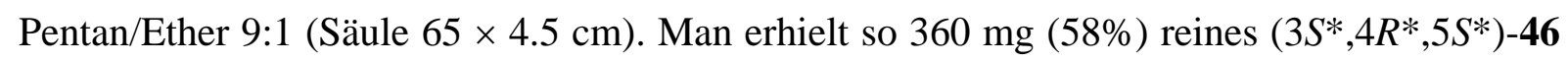
als farblosen glasartigen Feststoff vom Schmp. $78^{\circ} \mathrm{C}$.

$\mathrm{C}_{17} \mathrm{H}_{26} \mathrm{O}$ (246.4)

Ber.: C $82.87 \quad$ H 10.64

Gef.: C $82.61 \quad$ H 10.48

IR (Abb. 23) (KBr)

${ }^{1} \mathbf{H}-N M R$ (Abb. 60) (600 MHz, $\mathrm{C}_{6} \mathrm{D}_{6}, \mathrm{C}_{6} \mathrm{D}_{5} \mathrm{H}$ int): $\delta=1.20-1.67$ ppm (m, 19H), 1.73-1.79 (m, 1H), 1.90-1.99 (m, 1H), 2.08-2.19 (m, 2H), 2.43 (d, J = 4.5 Hz, 1H), 2.54-2.68 (m, 1H), $2.83(\mathrm{~d}, \mathrm{~J}=4.5 \mathrm{~Hz}, 1 \mathrm{H})$.

${ }^{13}$ C-NMR (Abb. 101) (150.8 MHz, $\left.\mathrm{C}_{6} \mathrm{D}_{6}, \mathrm{C}_{6} \mathrm{D}_{6} \mathrm{int}\right): \delta=20.30 \mathrm{ppm}(\mathrm{t}), 21.30(\mathrm{t}), 22.85(\mathrm{t})$, $23.68(\mathrm{t}), 23.89(\mathrm{t}), 26.13(\mathrm{t}), 33.80(\mathrm{t}), 35.16(\mathrm{t}), 36.65(\mathrm{t}), 37.17(\mathrm{t}), 37.19(\mathrm{t}), 40.83(\mathrm{t}), 53.97$ (t), $55.26(\mathrm{~s}), 58.13(\mathrm{~s}), 58.68(\mathrm{~s}), 65.51(\mathrm{~s})$.

MS (EI): $m / e=246\left(3, \mathrm{M}^{+}\right), 108(100)$.

\section{$\left(5 S^{*}, 6 S^{*}\right)$-Trispiro[4.0.0.4.3.3]heptadecan-2-on $\left[\left(5 S^{*}, 6 S^{*}\right)-35\right]$}

Lithiumiodid (1.98 g, $14.8 \mathrm{mmol}$ ) wurde $2 \mathrm{~h}$ bei $120^{\circ} \mathrm{C} / 0.1$ Torr getrocknet und anschließend in wasserfreiem THF $(14.8 \mathrm{ml})$ gelöst (exothermer Effekt). $0.98 \mathrm{ml}(0.98 \mathrm{mmol})$ dieser

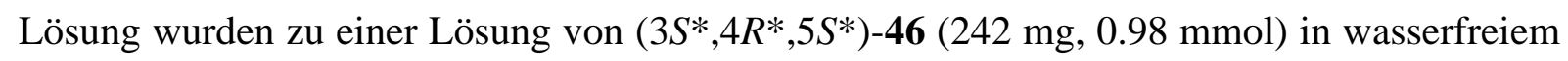
THF (4 ml) gegeben. Nach $6 \mathrm{~h}$ bei $60-70^{\circ} \mathrm{C}$ zeigte eine begleitende DC-Analyse

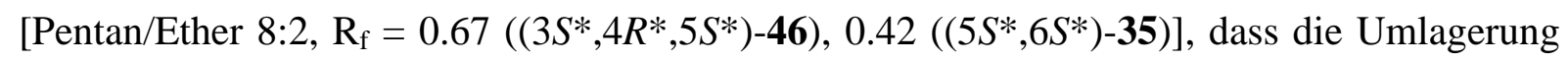
beendet war. Die Mischung wurde mit Pentan $(4 \mathrm{ml})$ verdünnt und mit Wasser $(4 \mathrm{ml})$ gewaschen. Die wässrige Phase wurde mit Pentan $(3 \times 5 \mathrm{ml})$ extrahiert und die vereinigten organischen Phasen wurden mit Wasser $(2 \times 8 \mathrm{ml})$ gewaschen und über Magnesiumsulfat getrocknet. Die Lösungsmittel wurden im Rotationsverdampfer abgezogen (Badtemperatur $45^{\circ} \mathrm{C} / 15$ Torr) und der Rückstand an Kieselgel in Pentan/Ether 8:2 chromatographiert (Säule $\left.95 \times 2.5 \mathrm{~cm}, \mathrm{R}_{\mathrm{f}}=0.42\right)$. Man erhielt so $213 \mathrm{mg}(88 \%)\left(5 S^{*}, 6 S^{*}\right)-35$ als farblose Flüssigkeit. Laut GC [Säule B, $230^{\circ} \mathrm{C}$; Retentionszeit (min): $9.58\left(\left(5 S^{*}, 6 S^{*}\right)-35\right)$ ] war das Material >99\% rein.

$\mathrm{C}_{17} \mathrm{H}_{26} \mathrm{O}$ (246.4)

Ber.: C $82.87 \quad$ H 10.64

Gef.: C $82.67 \quad$ H 10.42

IR (Abb. 18) (Film):1745 cm-1 (C=O). 
${ }^{1} \mathbf{H}-\mathbf{N M R}$ (Abb. 55) (600 MHz, $\mathrm{C}_{6} \mathrm{D}_{6}, \mathrm{C}_{6} \mathrm{D}_{5} \mathrm{H}$ int): $\delta=1.08-1.13 \mathrm{ppm}$ (m, 1H), 1.15-1.27 (m, 4H), 1.28-1.58 (m, 16H), 1.80-1.94 (m, 2H), 1.94-2.01 (m, 1H), 1.96 (d, J = 17 Hz, 1H), 2.17 (d, $\mathrm{J}=17 \mathrm{~Hz}, 1 \mathrm{H})$.

${ }^{13}$ C-NMR (Abb. 96) (150.8 MHz, $\mathrm{C}_{6} \mathrm{D}_{6}, \mathrm{C}_{6} \mathrm{D}_{6}$ int): $\delta=19.31 \mathrm{ppm}(\mathrm{t}), 19.73(\mathrm{t}), 23.66(\mathrm{t})$, $24.58(\mathrm{t}), 33.27(\mathrm{t}), 33.41(\mathrm{t}), 33.50(\mathrm{t}), 35.35(\mathrm{t}), 35.50(\mathrm{t}), 36.57(\mathrm{t}), 40.06(\mathrm{t}), 40.83(\mathrm{t}), 46.90$ (t), $51.90(\mathrm{~s}), 56.22(\mathrm{~s}), 56.52(\mathrm{~s}), 216.81(\mathrm{~s})$.

MS (EI): $m / e=246\left(3, \mathrm{M}^{+}\right), 67(100)$.

\section{rac-Trispiro[4.0.0.4.3.3]heptadecan (rac-8)}

$\mathrm{Zu}$ einem Gemisch aus fein gepulvertem Kaliumhydroxid (224 mg, $4.0 \mathrm{mmol}$ ) und Hydrazinhydrat (150 mg, $3.0 \mathrm{mmol}$ ) in Diethylenglykol (2.5 ml) wurde (5S*,6S*)-35 (123 mg, $0.50 \mathrm{mmol}$ ) gegeben und die Mischung unter Argon und Rühren auf $180^{\circ} \mathrm{C}$ erhitzt. Nach 2 h war die Reaktion nach GC-Analyse [Säule B, $200^{\circ} \mathrm{C}$, rel. Retentionszeiten (min): 4.13

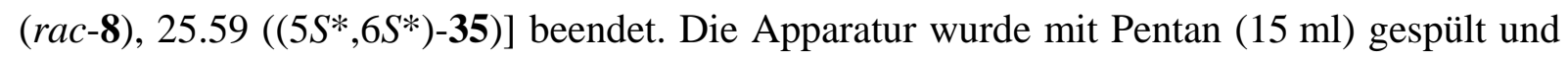
das Reaktionsgemisch in Wasser $(8 \mathrm{ml})$ gegossen und mit Pentan $(3 \times 8 \mathrm{ml})$ extrahiert. Die vereinigten organischen Phasen wurden mit Wasser $(2 \times 8 \mathrm{ml})$ gewaschen, getrocknet $\left(\mathrm{MgSO}_{4}\right)$ und über eine $30 \mathrm{~cm}$ Vigreux-Kolonne eingeengt (Badtemperatur bis $60^{\circ} \mathrm{C}$ ). Der Rückstand wurde an Kieselgel in Pentan chromatographiert (Säule $15 \times 1 \mathrm{~cm}, \mathrm{R}_{\mathrm{f}}=0.74$ ). Das Lösungsmittel wurde über eine $30 \mathrm{~cm}$ Vigreux-Kolonne abdestilliert. Letzte Reste wurden im Rotationsverdampfer (Badtemperatur $40^{\circ} \mathrm{C} / 15$ Torr) entfernt. Man erhielt $94 \mathrm{mg}$ (82\%) reines rac-8 als farblose Flüssigkeit.

$\mathbf{C}_{17} \mathbf{H}_{28}$ (232.4)

Ber.: C $87.86 \quad$ H 12.14

Gef.: C $88.06 \quad$ H 11.92

IR (Abb. 8) (Film)

${ }^{1} \mathbf{H}-\mathrm{NMR}$ (Abb. 45) (600 MHz, $\mathrm{CDCl}_{3}, \mathrm{CHCl}_{3}$ int): $\delta=1.28-1.36 \mathrm{ppm}$ (m, 6H), 1.45-1.67 (m, $18 \mathrm{H}), 1.67-1.73$ (m, 2H), 1.82-1.88 (m, 2H).

${ }^{13}$ C-NMR (Abb. 86) (150.8 MHz, $\mathrm{CDCl}_{3}, \mathrm{CDCl}_{3}$ int): $\delta=19.57 \mathrm{ppm}(\mathrm{t}), 23.39(\mathrm{t}), 24.32(\mathrm{t})$, $32.54(\mathrm{t}), 35.21(\mathrm{t}), 35.79(\mathrm{t}), 40.94(\mathrm{t}), 55.92(\mathrm{~s}), 56.65(\mathrm{~s})$.

MS (EI): $m / e=232\left(14, \mathrm{M}^{+}\right), 43(100)$.

\section{Cyclopropyliden-spiro[4.4]nonan (49)}

Zu einer Suspension von Natriumhydrid (33.6 g, $0.84 \mathrm{~mol}$ ) in wasserfreiem THF (1.20 l) gab man unter Argon und Rühren Cyclopropyltriphenylphosphoniumbromid (322 g, $0.84 \mathrm{~mol}$ ) und erhitzte auf $70^{\circ} \mathrm{C}$. Nach $4 \mathrm{~h}$ wurde 71 (83.3 g, $0.60 \mathrm{~mol}$ ) zugetropft und die Reaktion gas- 
chromatographisch überwacht [Säule A, $140^{\circ} \mathrm{C}$; Retentionszeiten (min): 8.21 (49), 10.97 (71)]. Nach 40 h bei $70^{\circ} \mathrm{C}$ war keine Umsatzänderung mehr beobachtbar. Die Reaktionsmischung wurde in Wasser gegossen $(1.60 \mathrm{l})$ und mit Pentan $(4 \times 500 \mathrm{ml})$ extrahiert, und die vereinigten organischen Phasen wurden mit Wasser $(4 \times 200 \mathrm{ml})$ und gesättigter Ammoniumchlorid $(200 \mathrm{ml})$ gewaschen und getrocknet $\left(\mathrm{MgSO}_{4}\right)$. Der größte Teil des Lösungsmittels wurde über eine $30 \mathrm{~cm}$ Vigreux-Kolonne abdestilliert (Badtemperatur bis $120^{\circ} \mathrm{C}$ ). Zwischenzeitlich ausgefallenes Triphenylphosphinoxid wurde mehrmals abfiltriert. Fraktionierung des Rückstandes lieferte 50.6 g (52\%) 49 als farblose Flüssigkeit vom Sdp. 85-95 C/14 Torr (Reinheit 97\% GC) sowie 11.9 g (14\%) unumgesetztes 71 (Sdp. $74^{\circ} \mathrm{C} / 14$ Torr, Reinheit $>99 \%$ GC).

\section{$\mathbf{C}_{13} \mathbf{H}_{20} \mathbf{O}(162.3)$}

Ber.: C $88.82 \quad$ H 11.18

Gef.: C 88.71

IR (Abb. 24) (Film)

${ }^{1}$ H-NMR (Abb. 61) (600 MHz, $\mathrm{C}_{6} \mathrm{D}_{6}, \mathrm{C}_{6} \mathrm{D}_{5} \mathrm{H}$ int): $\delta=0.80$ ppm (AA'-Teil eines AA'BB'Systems, 2H), 1.03 (BB'-Teil eines AA`BB'-Systems, 2H), 1.48-1.54 (m, 2H), 1.54-1.59 (m, 2H), 1.59-1.66 (m, 4H), 1.70-1.79 (m, 2H), 1.85-1.92 (m, 2H), 2.49 (m, $2 \mathrm{H})$.

${ }^{13}$ C-NMR (Abb. 102) (150.8 MHz, $\mathrm{C}_{6} \mathrm{D}_{6}, \mathrm{C}_{6} \mathrm{D}_{6}$ int): $\delta=-0.17 \mathrm{ppm}(\mathrm{t}), 2.39(\mathrm{t}), 24.25(\mathrm{t})$, $25.52(\mathrm{t}), 33.32(\mathrm{t}), 39.39(\mathrm{t}), 41.81(\mathrm{t}), 53.97(\mathrm{~s}), 108.77(\mathrm{~s}), 138.94(\mathrm{~s})$.

MS (EI): $m / e=162\left(4, \mathrm{M}^{+}\right), 91(100)$.

\section{(4S*)-Dispiro[3.0.4.3]dodecan-1-on [(4S*)-51]}

$\mathrm{Zu}$ einer Lösung von 49 (50.6 g, $0.31 \mathrm{~mol})$ in Dichlormethan (800 ml) wurde 3Chlorperbenzoesäure (116 g, 70\% w/w, $0.47 \mathrm{~mol}$ ) so zugegeben, dass die Innentemperatur $30^{\circ} \mathrm{C}$ nicht überschritt $(1.5 \mathrm{~h})$. Nach einer begleitenden DC-Analyse (Pentane/Ether 9:1; $\mathrm{R}_{\mathrm{f}}=$ 0.80 (49), 0.31 [(4S*)-51]) war die Reaktion nach 45 min beendet. 1 N KOH (600 ml) wurde zugegeben und die resultierende Mischung kräftig gerührt. Die Phasen wurden getrennt, die organische Phase wurde mit Wasser $(4 \times 250 \mathrm{ml})$ gewaschen, getrocknet $\left(\mathrm{MgSO}_{4}\right)$ und destillativ bis auf $400 \mathrm{ml}$ eingeengt. Nach Abkühlen tropfte man unter Argon und Rühren Bortrifluorid-Etherat $(0.3 \mathrm{ml})$ so zu, dass die Innentemperatur $30^{\circ} \mathrm{C}$ nicht überschritt. Mit den letzten Tropfen war die exotherme Reaktion und, nach DC, die Umlagerung beendet. Das Gemisch wurde mit festen Natriumcarbonat (30 g) neutralisiert, das Filtrat im Rotationsverdampfer eingeengt (Badtemperatur $40^{\circ} \mathrm{C} / 15$ Torr) und der Rückstand über eine Mikrodestille fraktioniert. Man erhielt 35.9 g (65\%) (4S*)-51 als farblose Flüssigkeit vom Sdp. 104$109^{\circ} \mathrm{C} / 12$ Torr. Laut GC [Säule B, $200^{\circ} \mathrm{C}$; Retentionszeit (min): 2.56 ((4S*)-51)] war das Material 97\% rein. 


\section{$\mathbf{C}_{12} \mathbf{H}_{18} \mathbf{O}(178.3)$}

Ber.: C $80.85 \quad$ H 10.18

Gef.: C 81.12 H 9.89

IR (Abb. 25) (Film): $1770 \mathrm{~cm}^{-1}(\mathrm{C}=\mathrm{O})$.

${ }^{1} \mathbf{H}-\mathbf{N M R}$ (Abb. 62) (600 MHz, $\mathrm{CDCl}_{3}, \mathrm{CHCl}_{3}$ int): $\delta=1.29-1.34$ ppm (m, 2H), 1.36-1.42 (m, 1H), 1.45-1.70 (m, 10H), 1.74 (ddd, $J=11,10.5,6.5$ Hz, 1H), 1.96 (ddd, J = 11, 11, $7 \mathrm{~Hz}$, 1H), 1.99-2.05 (m, 1H), 2.69 (ddd, $\mathrm{J}=17.5,11,6.5 \mathrm{~Hz}, 1 \mathrm{H}$ ), 2.87 (ddd, $\mathrm{J}=17.5,10.5,7 \mathrm{~Hz}$, $1 \mathrm{H})$.

${ }^{13}$ C-NMR (Abb. 103) (150.8 MHz, $\mathrm{CDCl}_{3}, \mathrm{CDCl}_{3}$ int): $\delta=19.21 \mathrm{ppm}(\mathrm{t}), 20.53(\mathrm{t}), 24.26(\mathrm{t})$, $25.01(\mathrm{t}), 33.28(\mathrm{t}), 33.82(\mathrm{t}), 34.19(\mathrm{t}), 37.64(\mathrm{t}), 42.28(\mathrm{t}), 54.45(\mathrm{~s}), 76.34(\mathrm{~s}), 216.47(\mathrm{~s})$.

MS (EI): $m / e=178\left(20, \mathrm{M}^{+}\right), 122(100)$.

\section{$\left(1 R^{*}, 4 S^{*}\right)-1-C y c l o p e n t-1-e n y l-d i s p i r o[3.0 .4 .3]$ dodecan-1-ol $\left[\left(1 R^{*}, 4 S^{*}\right)-52\right]$}

$\mathrm{Zu}$ einer Lösung von 1-Bromcyclopenten ${ }^{76}$ (10.6 g, $\left.72 \mathrm{mmol}\right)$ in wasserfreiem THF (240 ml) tropfte man innerhalb $1 \mathrm{~h}$ bei $-78^{\circ} \mathrm{C}$ unter Argon und Rühren eine 1.5 molare Lösung von tert-Butyllithium in Pentan (96 ml, $144 \mathrm{mmol}$ ). Nach weiteren $45 \mathrm{~min}$ bei $-78^{\circ} \mathrm{C}$ ließ man auf $0^{\circ} \mathrm{C}$ erwärmen. Die resultierende 0.21 molare Lösung von 1-Lithiocyclopenten $(340 \mathrm{ml})$ wurde in nächsten Schritt verwendet. Eine Suspension von gepulvertem trockenem $\mathrm{CeCl}_{3}$ (14.8 g, $60 \mathrm{mmol})$ in wasserfreiem THF $(220 \mathrm{ml})$ wurde $2 \mathrm{~h}$ bei Raumtemperatur unter Argon gerührt, mit (4S*)-51 (5,34 g, $30 \mathrm{mmol})$ versetzt und für weitere $2 \mathrm{~h}$ bei Raumtemperatur gerührt. Anschließend wurde das Gemisch auf $0^{\circ} \mathrm{C}$ gekühlt und mit $230 \mathrm{ml}$ (48 mmol) der 0.21 molaren Lösung von 1-Lithiocyclopenten versetzt. Man ließ auf Raumtemperatur erwärmen und überwachte die Reaktion durch GC [Säule B, $200^{\circ} \mathrm{C}$; Retentionszeiten (min): 2.56 ((4S*)-51), $\left.5.90\left(\left(1 R^{*}, 4 S^{*}\right)-52\right)\right]$. Nach 16 h enthielt das Gemisch 28\% nicht umgesetztes (4S*)-51. Daraufhin wurden die restlichen $110 \mathrm{ml}$ (23 mmol) der 0.21 molaren Lösung von 1Lithiocyclopenten zugegeben, und nach weiteren 30 min war der Umsatz komplett. Die Mischung wurde mit Pentan (300 ml) verdünnt und mit gesättigter Ammoniumchloridlösung (60 ml) hydrolysiert. Die überstehende Lösung wurde abdekantiert, der Rückstand mit Pentan $(2 \times 150 \mathrm{ml})$ extrahiert, und die vereinigten organischen Phasen wurden mit Wasser $(2 \times 300$ $\mathrm{ml})$ gewaschen und getrocknet $\left(\mathrm{MgSO}_{4}\right)$. Die Lösungsmittel wurden im Rotationsverdampfer abdestilliert (Badtemperatur $40^{\circ} \mathrm{C} / 15$ Torr) und der braune flüssige Rückstand (8.8 g) wurde an Kieselgel in Pentan/Ether 9:1 chromatographiert [Säule $100 \times 7 \mathrm{~cm}, \mathrm{R}_{\mathrm{f}}=0.46\left(\left(1 R^{*}, 4 S^{*}\right)\right.$ 52)]. Man erhielt $6.06 \mathrm{~g}(82 \%)\left(1 R^{*}, 4 S^{*}\right)-52$ als farblose Flüssigkeit (Reinheit 96\% GC).

$\mathrm{C}_{17} \mathrm{H}_{26} \mathrm{O}(246.4)$

Ber.: C 82.87 H 10.64

Gef.: C 81.64 H 10.33 
HRMS $m / e\left(\mathrm{M}^{+}\right) \quad$ Ber.: 246.1984 Gef.: 246.1984

IR (Abb. 26) (Film):3600 (OH), 3580-3350 cm $\left(\mathrm{OH}_{\mathrm{ass}}\right)$.

${ }^{1} \mathbf{H}-\mathbf{N M R}$ (Abb. 63) (600 MHz, $\mathrm{C}_{6} \mathrm{D}_{6}, \mathrm{C}_{6} \mathrm{D}_{5} \mathrm{H}$ int): $\delta=0.94-0.97 \mathrm{ppm}(\mathrm{m}, 1 \mathrm{H}), 1.32-1.60$ (m, 8H), 1.61-1.72 (m, 4H), 1.73-1.84 (m, 4H), 1.89-1.95 (m, 1H), $2.15\left(\mathrm{~m}_{\mathrm{c}}, 1 \mathrm{H}\right), 2.22-2.28$ (m, $3 \mathrm{H}), 2.31\left(\mathrm{~m}_{\mathrm{c}}, 1 \mathrm{H}\right), 2.35-2.45$ (m, 2H), 5.43 (dddd, $\mathrm{J}=2$, 2, 2, $\left.2 \mathrm{~Hz}, 1 \mathrm{H}\right)$.

${ }^{13}$ C-NMR (Abb. 104) (150.8 MHz, $\left.\mathrm{C}_{6} \mathrm{D}_{6}, \mathrm{C}_{6} \mathrm{D}_{6} \mathrm{int}\right): \delta=19.48 \mathrm{ppm}(\mathrm{t}), 24.37$ (t), 25.12 (t), $25.61(\mathrm{t}), 26.68(\mathrm{t}), 31.86(\mathrm{t}), 32.60(\mathrm{t}), 33.39(\mathrm{t}), 34.16(\mathrm{t}), 35.51(\mathrm{t}), 36.32(\mathrm{t}), 38.86(\mathrm{t}), 55.18$ (s), 58.21 (s), 81.24 (s), 125.41 (d), 149.40 (s).

MS (EI): $m / e=246\left(1, \mathrm{M}^{+}\right), 110(100)$.

\section{$\left(6 S^{*}, 7 S^{*}, 8 R^{*}\right)-8$-Brom-trispiro[4.0.0.4.3.3]heptadecan-12-on $\left[\left(6 S^{*}, 7 S^{*}, 8 R^{*}\right)-54\right]$}

Zu trocknem Zinkbromid (1.08 g, $4.8 \mathrm{mmol}$ ) in wasserfreiem Acetonitril (22 ml) gab man trockenes N-Chlor-toluol-4-sulfonsäureamid (1.09 g, $4.8 \mathrm{mmol}$ ) und rührte unter Argon bis eine nahezu klare gelbe Lösung vorlag (10 min). Diese Lösung gab man unter Argon und Rühren zu (1 $\left.R^{*}, 4 S^{*}\right)-52$ (984 mg, $\left.4.0 \mathrm{mmol}\right)$. Es bildete sich ein Niederschlag und die Mischung wurde farblos. Nach 15 min war die Umsetzung laut DC [Pentan/Ether 8:2; $\mathrm{R}_{\mathrm{f}}=$ $\left.0.62\left(\left(1 R^{*}, 4 S^{*}\right)-52\right), 0.45\left(\left(6 S^{*}, 7 S^{*}, 8 R^{*}\right)-54\right)\right]$ beendet. Die Mischung wurde über Kieselgel (Säule $8 \times 4.5 \mathrm{~cm}$ ) filtriert und mit Ether eluiert. Die Lösungsmittel wurden abdestilliert (Badtemperatur $40^{\circ} \mathrm{C} / 15$ Torr $)$ und der heterogene Rückstand mit Pentan $(2 \times 25$ ml) extrahiert. Die vereinigten Extrakte wurden eingeengt (Badtemperatur $40^{\circ} \mathrm{C} / 15$ Torr) und der Rückstand (1.34 g) an Kieselgel in Pentan/Ether 8:2 chromatographiert (Säule $70 \times 4.5 \mathrm{~cm}$ ). Man erhielt $1.07 \mathrm{~g}(82 \%)$ reines $\left(6 S^{*}, 7 S^{*}, 8 R^{*}\right)-54$ als farblosen Feststoff vom Schmp. $76^{\circ} \mathrm{C}$.

$\mathrm{C}_{17} \mathrm{H}_{25} \mathrm{BrO}$ (325.3)

Ber.: C $62.77 \quad$ H 7.75

Gef.: C $62.85 \quad$ H 7.64

IR (Abb. 28) (KBr): $1730 \mathrm{~cm}^{-1}(\mathrm{C}=\mathrm{O})$.

${ }^{1} \mathbf{H}-N M R$ (Abb. 65) (600 MHz, $\mathrm{C}_{6} \mathrm{D}_{6}, \mathrm{C}_{6} \mathrm{D}_{5} \mathrm{H}$ int): $\delta=1.03-1.15$ ppm (m, 3H), 1.18-1.27 (m, 2H), 1.30-1.51 (m, 10H), 1.52-1.67 (m, 3H), 1.85 (m, $1 \mathrm{H}), 1.89-2.07$ (m, 3H), 2.15 (ddd, $\mathrm{J}=$ 18.5, 10, $10 \mathrm{~Hz}, 1 \mathrm{H}), 2.47-2.56$ (m, 1H), 4.11 (ddd, $\mathrm{J}=8,8,2.5 \mathrm{~Hz}, 1 \mathrm{H}$ ).

${ }^{13}$ C-NMR (Abb. 106) (150.8 MHz, $\left.\mathrm{C}_{6} \mathrm{D}_{6}, \mathrm{C}_{6} \mathrm{D}_{6} \mathrm{int}\right): \delta=19.88 \mathrm{ppm}(\mathrm{t}), 23.21(\mathrm{t}), 23.74(\mathrm{t})$, $24.34(t), 28.77(t), 31.41(t), 33.94(t), 34.69(t), 35.65(t), 35.84(t), 37.39(t), 40.15(t), 53.56$ (d), 56.59 (s), 56.74 (s), 65.09 (s), 215.82 (s).

MS (EI): $m / e=326,324\left(9,9, \mathrm{M}^{+}\right), 108$ (100). 
$\left(6 S^{*}\right)$-Trispiro[4.0.0.4.3.3]heptadecan-12-on [(6S*)-55]

Ein Gemisch aus (6S*,7S*,8R*)-54 (976 mg, $3.00 \mathrm{mmol})$ und Zinkstaub (1.11 g, $17 \mathrm{mmol})$ in Eisessig (3.0 ml) wurde unter Argon und Rühren auf $60^{\circ} \mathrm{C}$ erhitzt. Nach GC-Analyse [Säule

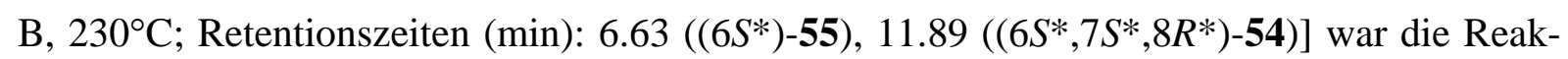
tion nach $1.5 \mathrm{~h}$ beendet. Die Mischung wurde mit Wasser (40 ml) verdünnt und mit Pentan (4 x $30 \mathrm{ml}$ ) extrahiert. Die vereinigten Extrakte wurden mit gesättigter Natriumhydrogencarbonatlösung $(80 \mathrm{ml})$ und Wasser $(2 \times 80 \mathrm{ml})$ gewaschen, getrocknet $\left(\mathrm{MgSO}_{4}\right)$ und im Rotationsverdampfer (Badtemperatur $40^{\circ} \mathrm{C} / 15$ Torr) eingeengt. Es verblieben $655 \mathrm{mg}$ (83\%) rohes (6S*)-55 als farbloser Feststoff vom Schmp. 61ํㅡ (Reinheit 94\% GC). Dieses Material wurde als solches weiter umgesetzt. Kristallisation aus Aceton durch Diffusion mit Wasser lieferte analytisch reine farblose Kristalle vom Schmp. $67-68^{\circ} \mathrm{C}$.

$\mathrm{C}_{17} \mathrm{H}_{26} \mathrm{O}$ (246.4)

Ber.: C $82.87 \quad$ H 10.64

Gef.: C 82.68 H 10.65

IR (Abb. 29) (KBr): $1730 \mathrm{~cm}^{-1}(\mathrm{C}=\mathrm{O})$.

${ }^{1} \mathbf{H}-N M R$ (Abb. 66) (600 MHz, $\mathrm{C}_{6} \mathrm{D}_{6}, \mathrm{C}_{6} \mathrm{D}_{5} \mathrm{H}$ int): $\delta=1.10-1.16 \mathrm{ppm}$ (m, 1H), 1.20-1.35 (m, 5H), 1.37-1.63 (m, 14H), 1.64-1.74 (m, 2H), 1.89-1.99 (m, 2H), $2.02\left(\mathrm{~m}_{\mathrm{c}}, 1 \mathrm{H}\right), 2.14$ (ddd, $\mathrm{J}=$ 19, 10, $2 \mathrm{~Hz}, 1 \mathrm{H})$.

${ }^{13}$ C-NMR (Abb. 107) (150.8 MHz, $\mathrm{C}_{6} \mathrm{D}_{6}, \mathrm{C}_{6} \mathrm{D}_{6}$ int): $\delta=20.32 \mathrm{ppm}(\mathrm{t}), 23.28(\mathrm{t}), 24.00(\mathrm{t})$, $25.22(\mathrm{t}), 25.48(\mathrm{t}), 28.74(\mathrm{t}), 29.65(\mathrm{t}), 31.11(\mathrm{t}), 32.79(\mathrm{t}), 33.09(\mathrm{t}), 34.70(\mathrm{t}), 36.39(\mathrm{t}), 40.06$ (t), $54.95(\mathrm{~s}), 56.11(\mathrm{~s}), 63.32(\mathrm{~s}), 218.95(\mathrm{~s})$.

MS (EI): $m / e=246\left(100, \mathrm{M}^{+}\right)$.

\section{rac-Trispiro[4.0.0.4.3.3]heptadecan (rac-8)}

Zu einer Lösung von Natrium (276 mg, $12.0 \mathrm{mmol}$ ) in Diethylenglykol (16 ml) gab man (6S*)-55 (246 mg, $1.0 \mathrm{mmol}$ ) und wasserfreies Hydrazin (2.60 g, $81 \mathrm{mmol}$ ) und erhitzte unter Argon und Rühren auf $180^{\circ} \mathrm{C}$. Laut GC [Säule A, $230^{\circ} \mathrm{C}$; Retentionszeiten (min): 2.06 (rac8), 5.93 ((6S*)-55), 51.85 (Hydrazon)] war nach 48 h alles (6S*)-55 verbraucht. Der größte Teil des überschüssigen Hydrazins und des Reaktionswassers wurde unter strömendem Argon durch langsame Temperaturerhöhung auf $200-205^{\circ} \mathrm{C}$ abdestilliert. Nach 10 Tagen bei dieser Temperatur war die Reduktion vollständig. Die Apparatur wurde mit Pentan (20 ml) gespült und die Reaktionsmischung in Wasser $(50 \mathrm{ml})$ gegossen und mit Pentan $(2 \times 50 \mathrm{ml})$ extrahiert. Die vereinigten organischen Phasen wurden mit Wasser $(3 \times 50 \mathrm{ml})$ gewaschen und getrocknet $\left(\mathrm{MgSO}_{4}\right)$. Das Lösungsmittel wurde abdestilliert (Badtemperatur $35^{\circ} \mathrm{C} / 15$ Torr) und der Rückstand (150 mg) über Kieselgel filtriert [Säule $18 \times 1 \mathrm{~cm}, \mathrm{R}_{\mathrm{f}}=0.74$ (rac-8)] und mit Pentan eluiert. Man erhielt 119 mg (50\%) rac-8 als farblose Flüssigkeit (Reinheit 
96\% GC). Die ${ }^{1} \mathrm{H}$ - und ${ }^{13} \mathrm{C}-\mathrm{NMR}-\mathrm{Daten}$ stimmten mit den Daten des durch Deoxygenierung von $\left(5 S^{*}, 6 S^{*}\right)-35$ erhaltenen Materials überein.

\section{$\left(1 R^{*}, 4 S^{*}\right)-1-C y c l o b u t-1-e n y l-d i s p i r o[3.0 .4 .3]$ dodecan-1-ol $\left[\left(1 R^{*}, 4 S^{*}\right)-53\right]$}

Zu einer Lösung von 1-Bromcyclobuten ${ }^{42}$ (4.99 g, 37.5 mmol) in wasserfreiem THF (125 ml) tropfte man innerhalb $1 \mathrm{~h}$ bei $-78^{\circ} \mathrm{C}$ unter Argon und Rühren eine 1.5 molare Lösung von tert-Butyllithium in Pentan $(50 \mathrm{ml}, 75 \mathrm{mmol})$. Nach weiteren $45 \mathrm{~min}$ bei $-78^{\circ} \mathrm{C}$ ließ man auf $0^{\circ} \mathrm{C}$ erwärmen. Die resultierende Lösung von 1-Lithiocyclobuten wurde im nächsten Schritt verwendet. Eine Suspension von gepulvertem trockenem $\mathrm{CeCl}_{3}(7.38 \mathrm{~g}, 30 \mathrm{mmol})$ in wasserfreiem THF (110 ml) wurde $2 \mathrm{~h}$ bei Raumtemperatur unter Argon gerührt, mit (4S*)-51 (2.67 g, $15 \mathrm{mmol}$ ) versetzt und weitere $2 \mathrm{~h}$ bei Raumtemperatur gerührt. Anschließend wurde auf $0^{\circ} \mathrm{C}$ gekühlt und die gesamte Lösung von 1-Lithiocyclobuten innerhalb von 25 min zugetropft. Man ließ auf Raumtemperatur erwärmen und überwachte den Fortgang der Reaktion gaschromatographisch [Säule B, 200 $\mathrm{C}$; Retentionszeiten (min): 2.56 ((4S*)-51), 3.97 $\left.\left(\left(1 R^{*}, 4 S^{*}\right)-53\right)\right]$. Nach einer Stunde war alles $\left(4 S^{*}\right)-51$ verbraucht. Die Mischung wurde mit Pentan (150 ml) verdünnt und mit gesättigter Ammoniumchloridlösung (40 ml) hydrolysiert. Die überstehende Lösung wurde abdekantiert und der Rückstand mit Pentan $(2 \times 75 \mathrm{ml})$ extrahiert. Die vereinigten organischen Phasen wurden mit Wasser $(2 \times 150 \mathrm{ml})$ gewaschen und getrocknet $\left(\mathrm{MgSO}_{4}\right)$. Die Lösungsmittel wurden im Rotationsverdampfer abdestilliert (Badtemperatur $40^{\circ} \mathrm{C} / 15$ Torr) und der gelbe Rückstand (4.0 g) an Kieselgel in Pentan/Ether 9:1 chromatographiert [Säule $\left.75 \times 6 \mathrm{~cm}, \mathrm{R}_{\mathrm{f}}=0.43\left(\left(4 S^{*}\right)-51\right), 0.39\left(\left(1 R^{*}, 4 S^{*}\right)-53\right)\right]$. Man erhielt 2.23 g (64\%) $\left(1 R^{*}, 4 S^{*}\right)-53$ als farblose Flüssigkeit (Reinheit 96\% GC).

$$
\mathrm{C}_{\mathbf{1 6}} \mathrm{H}_{\mathbf{2 4}} \mathrm{O} \text { (232.4) }
$$

Ber.: C $82.70 \quad$ H 10.41

Gef.: C $81.75 \quad$ H 10.14

HRMS $m / e\left(\mathrm{M}^{+}\right) \quad$ Ber.: 232.1827 Gef.: 232.1827.

IR (Abb. 27) (Film): 3600 (OH), 3580-3300 $\mathrm{cm}^{-1}\left(\mathrm{OH}_{\mathrm{ass}}\right)$.

${ }^{1} \mathbf{H}-\mathbf{N M R}$ (Abb. 64) (600 MHz, $\mathrm{C}_{6} \mathrm{D}_{6}, \mathrm{C}_{6} \mathrm{D}_{5} \mathrm{H}$ int): $\delta=1.07$ ppm (br s, $1 \mathrm{H}$ ), 1.30-1.41 (m, $4 \mathrm{H}), 1.46-1.65(\mathrm{~m}, 7 \mathrm{H}), 1.72-1.85(\mathrm{~m}, 4 \mathrm{H}), 2.10-2.20(\mathrm{~m}, 2 \mathrm{H}), 2.23\left(\mathrm{~m}_{\mathrm{c}}, 2 \mathrm{H}\right), 2.36-2.50(\mathrm{~m}$, $3 \mathrm{H}), 5.67\left(\mathrm{~m}_{\mathrm{c}}, 1 \mathrm{H}\right)$.

${ }^{13}$ C-NMR (Abb. 105) (150.8 MHz, $\left.\mathrm{C}_{6} \mathrm{D}_{6}, \mathrm{C}_{6} \mathrm{D}_{6} \mathrm{int}\right): \delta=19.48 \mathrm{ppm}(\mathrm{t}), 25.40(\mathrm{t}), 25.49(\mathrm{t})$, $25.76(\mathrm{t}), 26.32(\mathrm{t}), 29.98(\mathrm{t}), 30.11(\mathrm{t}), 34.08(\mathrm{t}), 35.34(\mathrm{t}), 36.52(\mathrm{t}), 38.84(\mathrm{t}), 55.07(\mathrm{~s})$, 58.02 (s), 79.97 (s), 127.86 (d), 153.71 (s).

MS (EI): $m / e=232\left(2, \mathrm{M}^{+}\right), 96$ (100). 
$\left(1 R^{*}, 4 S^{*}, 5 S^{*}\right)-1-B r o m-t r i s p i r o[3.0 .0 .4 .3 .3]$ hexadecan-16-on $\left[\left(1 R^{*}, 4 S^{*}, 5 S^{*}\right)-56\right]$

Zu trocknem Zinkbromid (2.10 g, $9.31 \mathrm{mmol}$ ) in wasserfreiem Acetonitril (42 ml) gab man trockenes N-Chlor-toluol-4-sulfonsäureamid (2.12 g, $9.31 \mathrm{mmol}$ ) und rührte unter Argon, bis eine nahezu klare gelbe Lösung vorlag (10 min). Diese Lösung gab man unter Argon und Rühren zu $\left(1 R^{*}, 4 S^{*}\right)-53(1.78 \mathrm{~g}, 7.67 \mathrm{mmol})$. Es bildete sich ein Niederschlag und die Mischung wurde farblos. Nach $1.5 \mathrm{~h}$ war die Umsetzung laut DC [Pentan/Ether 95:5; $\mathrm{R}_{\mathrm{f}}=$ $\left.0.25\left(\left(1 R^{*}, 4 S^{*}\right)-53\right), 0.23\left(\left(1 R^{*}, 4 S^{*}, 5 S^{*}\right)-56\right)\right]$ beendet. Die Mischung wurde über Kieselgel (Säule $21 \times 4.5 \mathrm{~cm}$ ) filtriert und mit Ether eluiert. Die Lösungsmittel wurden abdestilliert (Badtemperatur $40^{\circ} \mathrm{C} / 15$ Torr) und der heterogene Rückstand (3.8 g) mit Pentan $(5 \times 25 \mathrm{ml})$ extrahiert. Die vereinigten Extrakte wurden eingeengt (Badtemperatur $40^{\circ} \mathrm{C} / 15$ Torr) und der Rückstand (2.36 g) an Kieselgel in Pentan/Ether 95:5 chromatographiert (Säule $75 \times 5.5 \mathrm{~cm}$ ). Man erhielt 1.58 g (66\%) (1 $\left.R^{*}, 4 S^{*}, 5 S^{*}\right)-56$ als farbloses Öl (Reinheit 90\% NMR), das unter Kühlung fest wurde. Schmp. $29^{\circ} \mathrm{C}$.

$\mathbf{C}_{16} \mathrm{H}_{23} \mathrm{BrO}$ (311.3)

Ber.: C $61.74 \quad$ H 7.75

Gef.: C $61.96 \quad$ H 7.23

HRMS $m / e\left(\mathrm{M}^{+}\right) \quad$ Ber.:310.0932 Gef.: 310.0932.

IR (Abb. 30) (Film): $1738 \mathrm{~cm}^{-1}(\mathrm{C}=\mathrm{O})$.

${ }^{1} \mathbf{H}-N M R$ (Abb. 67) (600 MHz, $\mathrm{C}_{6} \mathrm{D}_{6}, \mathrm{C}_{6} \mathrm{D}_{5} \mathrm{H}$ int): $\delta=0.91-0.99$ ppm (m, 2H), 0.99-1.14 (m, $2 \mathrm{H}), 1.18-1.41(\mathrm{~m}, 9 \mathrm{H}), 1.52-1.63(\mathrm{~m}, 4 \mathrm{H}), 1.92-2.02(\mathrm{~m}, 2 \mathrm{H}), 2.26\left(\mathrm{~m}_{\mathrm{c}}, 1 \mathrm{H}\right), 2.35\left(\mathrm{~m}_{\mathrm{c}}, 1 \mathrm{H}\right)$, $2.62\left(\mathrm{~m}_{\mathrm{c}}, 1 \mathrm{H}\right), 4.20$ (dd, $\left.\mathrm{J}=9,9 \mathrm{~Hz}, 1 \mathrm{H}\right)$.

${ }^{13}$ C-NMR (Abb. 108) (150.8 MHz, $\left.\mathrm{C}_{6} \mathrm{D}_{6}, \mathrm{C}_{6} \mathrm{D}_{6} \mathrm{int}\right): \delta=19.72 \mathrm{ppm}(\mathrm{t}), 22.69(\mathrm{t}), 23.05(\mathrm{t})$, $25.31(\mathrm{t}), 29.22(\mathrm{t}), 29.62(\mathrm{t}), 33.53(\mathrm{t}), 34.68(\mathrm{t}), 34.91(\mathrm{t}), 36.30(\mathrm{t}), 37.28(\mathrm{t}), 44.70(\mathrm{~d})$, $55.17(\mathrm{~s}), 56.85(\mathrm{~s}), 65.63(\mathrm{~s}), 215.24(\mathrm{~s})$.

MS (EI): $m / e=312,310\left(8,8, \mathrm{M}^{+}\right), 231$ (100).

\section{$\left(1 S^{*}, 4 S^{*}\right)-1-\left[\left(1^{\prime}-\right.\right.$ Methylselanyl)cyclobutyl)]dispiro[3.0.4.3]dodecan-1-ol [(1S*,4S*)-58]}

Zu einer Lösung von 1,1-Bis(methylselanyl)cyclobutan ${ }^{44}$ (2.70 g, $11.0 \mathrm{mmol}$ ) in wasserfreiem Ether $(11 \mathrm{ml})$ tropfte man bei $-78^{\circ} \mathrm{C}$ unter Argon und Rühren eine 1.5 molare Lösung von tert-Butyllithium in Pentan $(7.9 \mathrm{ml}, 11.8 \mathrm{mmol})$ so $\mathrm{zu}$, dass die Innentemperatur $60^{\circ} \mathrm{C}$ nicht überstieg $(1 \mathrm{~h})$. Eine Stunde nach beendeter Zugabe tropfte man innerhalb 10 min (4S*)-51 (1.60 g, $9.0 \mathrm{mmol}) \mathrm{zu}$ und hielt weiter bei $-78^{\circ} \mathrm{C}$. Nach einer weiteren Stunde war laut DC

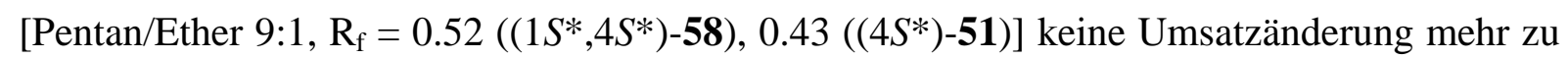
beobachten. Die Reaktionsmischung wurde noch kalt mit gesättigter Ammoniumchloridlösung $(5 \mathrm{ml})$ hydrolysiert, und die organische Phase wurde abgetrennt und getrocknet 
$\left(\mathrm{MgSO}_{4}\right)$. Die Lösungsmittel wurden abdestilliert (Badtemperatur $60^{\circ} \mathrm{C} / 20$ Torr) und der Rückstand (3.60 g) an Kieselgel in Pentan/Ether 9:1 chromatographiert (Säule $65 \times 4.5 \mathrm{~cm}$ ). Man erhielt $1.26 \mathrm{~g}$ (37\%) eines 85:15 Gemisches aus $\left(1 S^{*}, 4 S^{*}\right)$-58 und nicht umgesetztem (4S*)-51) als übelriechende farblose Flüssigkeit. Dieses Material wurde als solches weiter umgesetzt.

${ }^{1} \mathbf{H}-\mathbf{N M R}$ (Abb. 69) (600 MHz, $\mathrm{CDCl}_{3}, \mathrm{CHCl}_{3}$ int): $\delta=1.24-1.35$ ppm (m, 3H), 1.36-1.48 (m, $4 \mathrm{H}), 1.49-1.69(\mathrm{~m}, 6 \mathrm{H}), 1.77-1.99(\mathrm{~m}, 4 \mathrm{H}), 2.02-2.18(\mathrm{~m}, 3 \mathrm{H}), 2.12$ (s, 3H), 2.21-2.34 (m, $3 \mathrm{H}), 2.47\left(\mathrm{~m}_{\mathrm{c}}, 1 \mathrm{H}\right), 2.67-2.74(\mathrm{~m}, 1 \mathrm{H})$.

${ }^{13}$ C-NMR (Abb. 111) (150.8 MHz, $\mathrm{CDCl}_{3}, \mathrm{CDCl}_{3}$ int): $\delta=4.91 \mathrm{ppm}(\mathrm{q}), 16.62(\mathrm{t}), 19.06$ (t), $25.68(\mathrm{t}), 25.80(\mathrm{t}), 27.25(\mathrm{t}), 28.54(\mathrm{t}), 28.97(\mathrm{t}), 34.06(\mathrm{t}), 34.21(\mathrm{t}), 34.75(\mathrm{t}), 35.08(\mathrm{t}), 38.09$ (t), $55.22(\mathrm{~s}), 56.92(\mathrm{~s}), 58.50(\mathrm{~s}), 83.81(\mathrm{~s})$.

\section{(5S*)-Trispiro[3.0.0.4.3.3]hexadecan-16-on [(5S*)-57]}

Zu einer Lösung von 3-Chlorperbenzoesäure (2.94 g, 70\% w/w, $12.0 \mathrm{mmol}$ ) in Dichlormethan

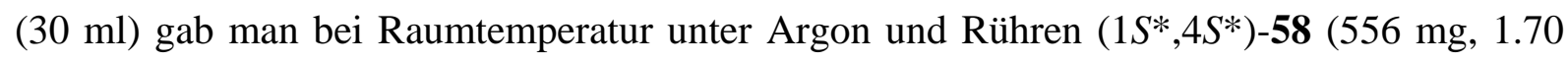
mmol). Nach 45 min war die Umsetzung laut DC [Pentan/Ether 9:1, $\mathrm{R}_{\mathrm{f}}=0.52\left(\left(1 S^{*}, 4 S^{*}\right)-58\right)$,

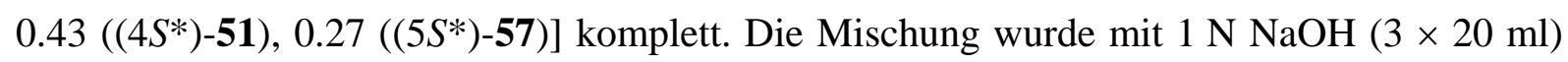
und Wasser $(30 \mathrm{ml})$ gewaschen und getrocknet $\left(\mathrm{MgSO}_{4}\right)$. Das Lösungsmittel wurde abdestilliert (Badtemperatur 30\%15 Torr) und der Rückstand (520 mg) an Kieselgel in Pentan/Ether 9:1 chromatographiert (Säule $45 \times 2.5 \mathrm{~cm}$ ). Man erhielt $236 \mathrm{mg}(70 \%)\left(5 S^{*}\right)-57$ als farblose Flüssigkeit. Laut GC [Säule B, $230^{\circ} \mathrm{C}$; Retentionszeit (min): 3.78 ((5S*)-57)] war das Material $>99 \%$ rein.

\section{$\mathbf{C}_{16} \mathbf{H}_{24} \mathbf{O}(232.4)$}

Ber.: C $82.70 \quad$ H 10.41

Gef.: C $82.76 \quad$ H 10.12 .

IR (Abb. 31) (Film): $1735 \mathrm{~cm}^{-1}(\mathrm{C}=\mathrm{O})$.

${ }^{1} \mathbf{H}-\mathbf{N M R}$ (Abb. 68) (600 MHz, $\mathrm{CDCl}_{3}, \mathrm{CHCl}_{3}$ int): $\delta=1.32-1.38$ ppm (m, 3H), 1.46-1.67 (m, $11 \mathrm{H}), 1.67-1.77$ (m, 2H), 1.84-1.98 (m, 3H), 2.04 (m $\left.\mathrm{m}_{\mathrm{c}}, 1 \mathrm{H}\right), 2.17-2.25$ (m, 3H), $2.29\left(\mathrm{~m}_{\mathrm{c}}, 1 \mathrm{H}\right)$.

${ }^{13}$ C-NMR (Abb. 109) (150.8 MHz, $\mathrm{CDCl}_{3}, \mathrm{CDCl}_{3}$ int): $\delta=14.75$ ppm (t), $19.72(\mathrm{t}), 22.63(\mathrm{t})$, $23.25(\mathrm{t}), 25.47(\mathrm{t}), 28.18(\mathrm{t}), 28.32(\mathrm{t}), 33.05(\mathrm{t}), 33.09(\mathrm{t}), 33.38(\mathrm{t}), 35.12(\mathrm{t}), 38.37(\mathrm{t}), 54.22$ (s), 56.35 (s), 58.41 (s), 221.10 (s).

MS (EI): m/e = 232 (30, $\left.\mathrm{M}^{+}\right), 123$ (100). 


\section{[15,15-D $\left.\mathrm{D}_{2}\right]-\left(5 S^{*}\right)-$ Trispiro[3.0.0.4.3.3]hexadecan-16-on $\left\{\left[15,15-\mathrm{D}_{2}\right]-\left(5 S^{*}\right)-57\right\}$}

Zu einer Lösung von (5S*)-57 (46 mg, $0.2 \mathrm{mmol})$ in THF (0.6 ml) gab man $2 \mathrm{~N} \mathrm{NaOD}$ in $\mathrm{D}_{2} \mathrm{O}$ (1.0 ml, $2.0 \mathrm{mmol}$ ) und rührte das resultierende Zweiphasengemisch bei Raumtemperatur kräftig. Nach 48 h wurde die wässrige Phase durch frische NaOD-Lösung ersetzt, und dieser Vorgang wurde fünfmal wiederholt. Die Mischung wurde mit Pentan (1.0 ml) verdünnt, und die organische Phase abgetrennt und getrocknet $\left(\mathrm{MgSO}_{4}\right)$. Die Lösungsmittel wurden abgezogen (Badtemperatur $40^{\circ} \mathrm{C} / 15$ Torr) und der Rückstand dickschichtchromatographisch gereinigt [SIL G-200 UV $254,20 \times 20 \times 0.2 \mathrm{~cm}$; Pentan/Ether 8:2, $\mathrm{R}_{\mathrm{f}}=0.44$ ([15,15- $\left.\left.\left.\mathrm{D}_{2}\right]-\left(5 S^{*}\right)-57\right)\right]$. Man erhielt $28 \mathrm{mg}(60 \%)$ [15,15-D $]-\left(5 S^{*}\right)-57$ (MS: 27\% $\mathrm{D}_{1}, 73 \%$ $\mathrm{D}_{2}$ ) als farblose Flüssigkeit.

${ }^{13}$ C-NMR (Abb. 110) (150.8 MHz, $\mathrm{CDCl}_{3}, \mathrm{CDCl}_{3}$ int): $\delta=14.72 \mathrm{ppm}(\mathrm{t}), 19.68(\mathrm{t}), 22.60(\mathrm{t})$, $23.21(\mathrm{t}), 25.42(\mathrm{t}), 27.97(\mathrm{t}), 28.28(\mathrm{t}), 32.71\left(\mathrm{tt}, \mathrm{J}=20,20 \mathrm{~Hz}, \mathrm{CD}_{2}\right), 33.02(\mathrm{t}, \mathrm{J}=20 \mathrm{~Hz}$, $\left.\mathrm{CD}_{1}\right), 33.03(\mathrm{t}), 33.05(\mathrm{t}), 35.08(\mathrm{t}), 38.32(\mathrm{t}), 54.17(\mathrm{~s}), 56.32(\mathrm{~s}), 58.39$ (s), $221.08(\mathrm{~s})$.

MS (EI): $m / e=233\left[34, \mathrm{M}^{+}\left({ }^{12} \mathrm{CD}_{1}\right)\right], 234\left[100, \mathrm{M}^{+}\left({ }^{13} \mathrm{CD}_{1},{ }^{12} \mathrm{CD}_{2}\right)\right], 235\left[18, \mathrm{M}^{+}\left({ }^{13} \mathrm{CD}_{2}\right)\right]$.

\section{(5S*)-Trispiro[3.0.0.4.3.3]hexadecan [(5S*)-15]}

Zu einer Lösung von Natrium (140 mg, $6.0 \mathrm{mmol}$ ) in Diethylenglykol (8 ml) gab man (5S*)57 (116 mg, $0.5 \mathrm{mmol}$ ) und wasserfreies Hydrazin (1.30 g, $40 \mathrm{mmol}$ ) und erhitzte unter Argon und Rühren auf $180^{\circ} \mathrm{C}$. Laut GC [Säule A, $230^{\circ} \mathrm{C}$; Retentionszeiten (min): 3.86 ((5S*)-15),

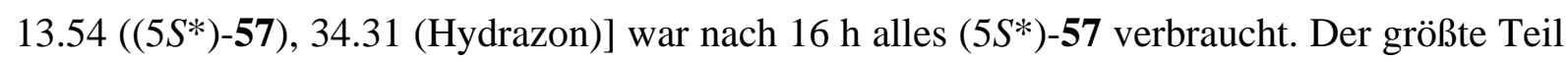
des überschüssigen Hydrazins und des Reaktionswassers wurde unter strömendem Argon und langsamer Temperaturerhöhung auf $200-205^{\circ} \mathrm{C}$ abdestilliert. Nach $72 \mathrm{~h}$ bei dieser Temperatur war die Reduktion vollständig. Die Apparatur wurde mit Pentan (30 ml) gespült und die Reaktionsmischung in Wasser $(50 \mathrm{ml})$ gegossen und mit Pentan $(2 \times 50 \mathrm{ml})$ extrahiert. Die vereinigten organischen Phasen wurden mit Wasser $(3 \times 50 \mathrm{ml})$ gewaschen und getrocknet $\left(\mathrm{MgSO}_{4}\right)$. Das Lösungsmittel wurde abdestilliert (Badtemperatur $25^{\circ} \mathrm{C} / 15$ Torr) und der Rückstand (95 mg) über Kieselgel filtriert [Säule $\left.10 \times 1 \mathrm{~cm}, \mathrm{R}_{\mathrm{f}}=0.77\left(\left(5 S^{*}\right)-15\right)\right]$ und mit Pentan eluiert. Man erhielt 87 mg (78\%) (5S*)-15 als farblose Flüssigkeit (Reinheit 97\% GC). Die ${ }^{1} \mathrm{H}$ - und ${ }^{13} \mathrm{C}$-NMR-Daten stimmten mit den Daten des durch Deoxygenierung von $\left(4 R^{*}, 5 S^{*}\right)-43$ erhaltenen Materials überein.

(SS,1R,4R)-(+)-[( $N$-Methyl-S-phenylsulfonimidoyl)methyl]-dispiro[3.0.4.3]dodecan-1-ol $[(\mathrm{S} S, 1 R, 4 R)-(+)-59] \quad$ und $(\mathrm{S} S, 1 S, 4 S)-(+)-[(N-M e t h y l-S$-phenylsulfonimidoyl)methyl]dispiro[3.0.4.3]dodecan-1-ol [(SS,1S,4S)-(+)-61]

$\mathrm{Zu}$ einer Lösung von (+)-(S)- $N, S$-Dimethyl-S-phenylsulfonimid ${ }^{48}$ (8.80 g, $50 \mathrm{mmol}$ ) in wasserfreiem THF $(150 \mathrm{ml})$ tropfte man bei $0^{\circ} \mathrm{C}$ unter Argon und Rühren eine 1.6 M Lösung 
von n-Butyllithium in Hexan (31.2 ml, $50 \mathrm{mmol}$ ). Die Mischung wurde noch $1 \mathrm{~h}$ bei $0^{\circ} \mathrm{C}$ gerührt und dann in der nächsten Stufe verwendet. Eine Suspension von gepulvertem trockenem $\mathrm{CeCl}_{3}$ (4.94 g, $20 \mathrm{mmol}$ ) in wasserfreiem THF (75 ml) wurde bei Raumtemperatur unter Argon 2 h kräftig gerührt. Nach Zugabe von (4S*)-51 (1.78 g, 10 mmol) wurde weitere 2 h bei Raumtemperatur gerührt. Anschließend wurde auf $-78^{\circ} \mathrm{C}$ gekühlt und die Lösung des lithierten N,S-Dimethyl-S-phenylsulfonimids innerhalb von 15 min zugetropft. Laut DC (Pentan/Ether 7:3; $\left.\mathrm{R}_{\mathrm{f}}=0.58 \quad\left[\left(4 S^{*}\right)-51\right], 0.33 \quad[(\mathrm{SS}, 1 R, 4 R)-59], 0.25 \quad[(\mathrm{SS}, 1 S, 4 S)-\mathbf{6 1}]\right)$ erhöhte sich der Umsatz nach $1 \mathrm{~h}$ bei $-78^{\circ} \mathrm{C}$ durch $1 \mathrm{~h}$ bei Raumtemperatur nicht. Die Mischung wurde mit Ether (300 ml) verdünnt und mit Wasser (10 ml) hydrolysiert. Die überstehende Lösung wurde abdekantiert, der Rückstand mit Ether (100 ml) extrahiert, und die vereinigten organischen Phasen wurden getrocknet $\left(\mathrm{MgSO}_{4}\right)$ und eingeengt (Badtemperatur $20^{\circ} \mathrm{C} / 15$ Torr). Der Rückstand (6.10 g) wurde an Kieselgel in Pentan/Ether 7:3 chromatographiert (Säule $70 \times 5 \mathrm{~cm}$ ). Man erhielt $0.26 \mathrm{~g}(15 \%)$ unumgesetztes (4S*)-51 als farblose Flüssigkeit, $1.28 \mathrm{~g}$ (37\%) (SS,1R,4R)-59 (Reinheit 99\% ${ }^{1} \mathrm{H}-\mathrm{NMR}, \geq 99 \%$ ee; $[\alpha]_{\mathrm{D}}{ }^{20}=+61.3$, c 1.07, $\mathrm{CHCl}_{3}$ ) als farblosen Feststoff vom Schmp. 61-63 $\mathrm{C}$, und $0.84 \mathrm{~g}(24 \%)(\mathrm{SS}, 1 \mathrm{~S}, 4 \mathrm{~S})-\mathbf{6 1}$ (Reinheit 99\% ${ }^{1} \mathrm{H}-\mathrm{NMR}, \geq 99 \%$ ee; $[\alpha]_{\mathrm{D}}{ }^{20}=+42.0$, c 1.06, $\mathrm{CHCl}_{3}$ ) als farblosen Feststoff vom Schmp. $70^{\circ} \mathrm{C}$. Beide Sulfonimide wurden aus Ethanol durch Diffusion von Wasser kristallisiert.

$(\mathrm{S} S, 1 R, 4 R)-(+)-[(N$-Methyl-S-phenylsulfonimidoyl)methyl]-dispiro[3.0.4.3]dodecan-1-ol $[(\mathrm{SS}, 1 R, 4 R)-(+)-59]$

$\mathrm{C}_{20} \mathrm{H}_{29} \mathrm{NO}_{2} \mathrm{~S}$ (347.5)

Ber.: C $69.12 \quad$ H 8.41

Gef.: C $68.99 \quad$ H 8.66

IR (Abb. 32) (KBr): $3600-3200 \mathrm{~cm}^{-1}\left(\mathrm{OH}_{\mathrm{ass}}\right)$.

${ }^{1} \mathbf{H}-\mathbf{N M R}$ (Abb. 70) (600 MHz, $\mathrm{CD}_{2} \mathrm{Cl}_{2}, \mathrm{CDHCl}_{2}$ int, $\left.-50^{\circ} \mathrm{C}\right): \delta=1.05-1.10 \mathrm{ppm}$ (br s, $1 \mathrm{H}$ ), 1.13-1.24 (m, 2H), 1.37-1.50 (m, 10H), 1.51-1.59 (m, 2H), $2.04\left(\mathrm{~m}_{\mathrm{c}}, 1 \mathrm{H}\right), 2.14\left(\mathrm{~m}_{\mathrm{c}}, 1 \mathrm{H}\right), 2.25-$ $2.32(\mathrm{~m}, 1 \mathrm{H}), 2.48(\mathrm{~s}, 3 \mathrm{H}), 2.55\left(\mathrm{~m}_{\mathrm{c}}, 1 \mathrm{H}\right), 3.22\left(\mathrm{AB}, \Delta v_{\mathrm{AB}}=45 \mathrm{~Hz}, \mathrm{~J}_{\mathrm{AB}}=13 \mathrm{~Hz}, 2 \mathrm{H}\right), 7.58(\mathrm{t}$, $\mathrm{J}=7.5 \mathrm{~Hz}, 2 \mathrm{H}), 7.64$ (t, J = 7.5 Hz, 1H), 7.84 (d, J = 7.5 Hz, 2H).

${ }^{13}$ C-NMR (Abb. 112) (150.8 MHz, $\mathrm{CDCl}_{3}, \mathrm{CDCl}_{3}$ int): $\delta=18.60 \mathrm{ppm}(\mathrm{t}), 24.75(\mathrm{t}), 24.91(\mathrm{t})$, $27.77(\mathrm{t}), 28.69(\mathrm{q}), 31.34(\mathrm{t}), 33.75(\mathrm{t}), 34.68(\mathrm{t}), 34.72(\mathrm{t}), 38.02(\mathrm{t}), 54.72(\mathrm{~s}), 58.26(\mathrm{~s})$, 62.32 (t), 78.52 (s), 128.95 (d), 129.65 (d), 133.29 (d), 138.38 (s).

MS (EI): $m / e=348\left(<1, \mathrm{M}^{+}\right), 125(100)$.

(SS,1S,4S)-(+)-[(N-Methyl-S-phenylsulfonimidoyl)methyl]-dispiro[3.0.4.3]dodecan-1-ol [(SS,1S,4S)-(+)-61]

$\mathrm{C}_{20} \mathrm{H}_{29} \mathrm{NO}_{2} \mathrm{~S}(347.5)$ 
Ber.: C $69.12 \quad$ H 8.41

Gef.: C $69.24 \quad$ H 8.50

IR (Abb. 34) (KBr): $3350-3100 \mathrm{~cm}^{-1}\left(\mathrm{OH}_{\mathrm{ass}}\right)$.

${ }^{1} \mathbf{H}$-NMR (Abb. 72) $\left(600 \mathrm{MHz}, \mathrm{CD}_{2} \mathrm{Cl}_{2}, \mathrm{CDHCl}_{2}\right.$ int, $\left.-50^{\circ} \mathrm{C}\right): \delta=1.06-1.24 \mathrm{ppm}(\mathrm{m}, 5 \mathrm{H})$, 1.27-1.33 (m, 1H), 1.35-1.48 (m, 5H), 1.49-1.61 (m, 5H), $1.96\left(\mathrm{~m}_{\mathrm{c}}, 1 \mathrm{H}\right), 2.24\left(\mathrm{~m}_{\mathrm{c}}, 2 \mathrm{H}\right), 2.63$ (s, 3H), $3.33\left(\mathrm{AB}, \Delta v_{\mathrm{AB}}=29 \mathrm{~Hz}, \mathrm{~J}_{\mathrm{AB}}=14 \mathrm{~Hz}, 2 \mathrm{H}\right), 7.55(\mathrm{t}, \mathrm{J}=7.5 \mathrm{~Hz}, 2 \mathrm{H}), 7.63(\mathrm{t}, \mathrm{J}=7.5$ $\mathrm{Hz}, 1 \mathrm{H}), 7.78$ (d, J = $7.5 \mathrm{~Hz}, 2 \mathrm{H})$.

${ }^{13}$ C-NMR (Abb. 114) (150.8 MHz, $\mathrm{CDCl}_{3}, \mathrm{CDCl}_{3}$ int): $\delta=18.66 \mathrm{ppm}(\mathrm{t}), 24.69$ (t), 24.90 (t), $27.08(\mathrm{t}), 28.81(\mathrm{q}), 32.41(\mathrm{t}), 33.50(\mathrm{t}), 34.53(\mathrm{t}), 34.75(\mathrm{t}), 37.92(\mathrm{t}), 54.80(\mathrm{~s}), 57.99(\mathrm{~s})$, 63.17 (t), 76.66 (s), 129.47 (d), 129.54 (d), 133.41 (d), 138.28 (s).

MS (EI): $m / e=348\left(1, \mathrm{M}^{+}\right), 211(100)$.

\section{(4R)-(+)Dispiro[3.0.4.3]dodecan-1-on [(4R)-(+)-51]}

(SS,1R,4R)-(+)-59 (1.10 g, $3.16 \mathrm{mmol})$ wurde unter Argon auf $110^{\circ} \mathrm{C}$ erhitzt. Nach DCAnalyse (Pentan/Ether 7:3; $\left.\mathrm{R}_{\mathrm{f}}=0.52[(4 R)-(+)-51], 0.30[(\mathrm{SS}, 1 R, 4 R)-(+)-59]\right)$ war die Reaktion nach $14 \mathrm{~h}$ beendet. Die Mischung wurde in Ether $(20 \mathrm{ml})$ aufgenommen, die Lösung eingeengt (Badtemperatur $20^{\circ} \mathrm{C} / 15$ Torr) und der Rückstand an Kieselgel in Pentan/Ether 7:3 chromatographiert (Säule $45 \times 2.5 \mathrm{~cm})$. Man erhielt $495 \mathrm{mg}(87 \%)(4 R)-(+)-51$ als farblose Flüssigkeit (Reinheit $99 \%,>99 \%$ ee; $[\alpha]_{\mathrm{D}}^{20}=+145$, c 1.21, Aceton; $\theta_{307}=+6328$, Methanol). Die chemische Reinheit wurde über GC bestimmt [Säule A, $230^{\circ} \mathrm{C}$; Retentionszeit (min): 3.71 [(4R)-(+)-51]. Die ${ }^{1} \mathrm{H}$ - und ${ }^{13} \mathrm{C}-\mathrm{NMR}-\mathrm{Daten}$ waren mit denen des Racemats identisch.

\section{(4S)-(-)-Dispiro[3.0.4.3]dodecan-1-on [(4S)-(-)-51]}

(SS,1S,4S)-(+)-61 (675 mg, 1.94 mmol) wurde wie für (SS,1R,4R)-(+)-59 beschrieben thermolysiert und aufgearbeitet. Man erhielt $331 \mathrm{mg}$ (96\%) (4S)-(-)-51 als farblose Flüssigkeit (Reinheit 99\%, $>99 \%$ ee; $[\alpha]_{\mathrm{D}}{ }^{20}=-145$, c 1.14 , Aceton; $\theta_{307}=-6538$, Methanol). Die ${ }^{1} \mathrm{H}-$ und ${ }^{13} \mathrm{C}-\mathrm{NMR}$-Daten waren mit denen des Racemats identisch.

Reduktion von (4S*)-51 mit (-)-DIP-Cl: (1S,4S)-(+)-Dispiro[3.0.4.3]dodecan-1-ol [(1S,4S)-(+)-62] und (1S,4R)-(+)-Dispiro[3.0.4.3]dodecan-1-ol [(1S,4R)-(+)-60]

Zu (-)-Diisopinocampheylchloroboran [(-)-DIP-Cl] (21.6 g, 67.4mmol) tropfte man innerhalb von 15 min unter Argon und Rühren (4S*)-51 (10.0 g, 56.2 mmol, Reinheit 97\%), wobei ein leicht exothermer Effekt und die Bildung einer klaren Lösung zu beobachten war. Nach GC-Analyse [Säule A, $230^{\circ} \mathrm{C}$; Retentionszeiten (min): 3.71 ((4S*)-51), 5.40 ((1S,4S)-(+)-62 und $(1 S, 4 R)-(+)-60)]$ war die Reduktion nach 18 h beendet. Die Reaktionsmischung wurde 
mit Ether (120 ml) verdünnt und mit Diethanolamin (13.0 g, 124 mmol) versetzt. Nach 2 h wurde der gebildete Feststoff abfitriert und mit Pentan $(2 \times 80 \mathrm{ml})$ gewaschen. Die vereinigten Filtrate wurden mit gesättigter Natriumhydrogencarbonatlösung $(100 \mathrm{ml})$ und Wasser $(2 \times$ $100 \mathrm{ml})$ gewaschen und getrocknet $\left(\mathrm{MgSO}_{4}\right)$. Die Lösungsmittel wurden abdestilliert (Badtemperatur $45^{\circ} \mathrm{C} / 15$ Torr) und der Rückstand (22.6 g) an Kieselgel zunächst in Pentan, und, nach Elution des vom Reagenz freigesetzten Pinens, in Pentan/Ether 8:2 chromatographiert [Säule $\left.80 \times 5.5 \mathrm{~cm}, \mathrm{R}_{\mathrm{f}}=0.34((1 S, 4 S)-(+)-62), 0.22((1 S, 4 R)-(+)-60)\right]$. Man erhielt $2.94 \mathrm{~g}$ (28\%) (1S,4S)-(+)-62 (Reinheit 96\% GC) und 2.27 g (21\%) (1S,4R)-(+)-60 (Reinheit 93\% GC) als farblose zähe Flüssigkeiten.

Analytisch reine Proben erhielt man durch präparative GC. $(1 S, 4 S)-(+)-62: 69 \%$ ee; $[\alpha]_{\mathrm{D}}{ }^{20}=$ +1.21 , c 1.24 , Aceton. $(1 S, 4 R)-(+)-60: \geq 99 \%$ ee; $[\alpha]_{\mathrm{D}}{ }^{20}=+22.1$, c 1.20 , Aceton. Die optischen Reinheiten entsprechen den optischen Reinheiten der durch Oxidation erhaltenen Ketone (4S)-(-)-51 (69\% ee) bzw. (4R)-(+)-51 ( $\geq 99 \%$ ee) (s. unten). Diese wiederum wurden aus den spezifischen Drehungen der durch Racematspaltung von $\left(4 S^{*}\right)-51$ mit (+)-(S)-N,SDimethyl-S-phenylsulfonimid gewonnenen enantiomerenreinen Ketone ermittelt.

\section{$\mathbf{C}_{12} \mathbf{H}_{20} \mathbf{O}(180.3)$}

Ber.: C $79.94 \quad$ H 11.18

Gef.: C $79.67 \quad$ H 10.97

\section{$(1 S, 4 S)$-Dispiro[3.0.4.3]dodecan-1-ol [(1S,4S)-(+)-62]}

HRMS m/e $\left(\mathrm{M}^{+}\right) \quad$ Ber.: 180.1514 Gef.: 180.1514 .

IR (Abb. 35) (Film): 3620 (OH), 3600-3100 cm $\mathrm{cm}^{-1}\left(\mathrm{OH}_{\mathrm{ass}}\right)$.

${ }^{1} \mathbf{H}-N M R$ (Abb. 73) (600 MHz, $\mathrm{CDCl}_{3}, \mathrm{CHCl}_{3}$ int): $\delta=1.27-1.33 \mathrm{ppm}(\mathrm{m}, 1 \mathrm{H}), 1.41\left(\mathrm{~m}_{\mathrm{c}}\right.$, 1H), 1.45-1.69 (m, 14H), 1.71-1.78 (m, 1H), $1.85\left(\mathrm{~m}_{\mathrm{c}}, 1 \mathrm{H}\right), 2.23\left(\mathrm{~m}_{\mathrm{c}}, 1 \mathrm{H}\right), 4.11\left(\mathrm{~m}_{\mathrm{c}}, 1 \mathrm{H}\right)$.

${ }^{13}$ C-NMR (Abb. 115) (150.8 MHz, $\mathrm{CDCl}_{3}, \mathrm{CDCl}_{3}$ int): $\delta=19.29$ ppm (t), 22.60 (t), $24.11(\mathrm{t})$, $25.70(\mathrm{t}), 28.21(\mathrm{t}), 33.18(\mathrm{t}), 34.70(\mathrm{t}), 37.86(\mathrm{t}), 39.36(\mathrm{t}), 53.53(\mathrm{~s}), 56.69(\mathrm{~s}), 77.21(\mathrm{~d})$.

MS (EI): $m / e=180\left(<1, \mathrm{M}^{+}\right), 95(100)$.

\section{(1S,4R)-Dispiro[3.0.4.3]dodecan-1-ol [(1S,4R)-(+)-60]}

IR (Abb. 33) (Film): 3600-3100 $\mathrm{cm}^{-1}\left(\mathrm{OH}_{\mathrm{ass}}\right)$.

${ }^{1} \mathbf{H}$-NMR (Abb. 71) (600 MHz, $\mathrm{CDCl}_{3}, \mathrm{CHCl}_{3}$ int): $\delta=1.20-1.45$ ppm (m, 8H), 1.51-1.69 (m, 8H), 1.76-1.85 (br s, 1H), $2.01\left(\mathrm{~m}_{\mathrm{c}}, 1 \mathrm{H}\right), 2.08$ (m, $\left.1 \mathrm{H}\right), 3.99$ (dd, J= 8, $\left.8 \mathrm{~Hz}, 1 \mathrm{H}\right)$.

${ }^{13}$ C-NMR (Abb. 113) (150.8 MHz, $\mathrm{CDCl}_{3}, \mathrm{CDCl}_{3}$ int): $\delta=19.69 \mathrm{ppm}(\mathrm{t}), 21.36(\mathrm{t}), 24.46(\mathrm{t})$, $24.58(\mathrm{t}), 27.86(\mathrm{t}), 29.89(\mathrm{t}), 32.88$ (t), $33.64(\mathrm{t}), 37.14(\mathrm{t}), 54.02$ (s), $58.06(\mathrm{~s}), 68.77$ (d).

MS (EI): $m / e=180\left(<1, \mathrm{M}^{+}\right), 121(100)$. 


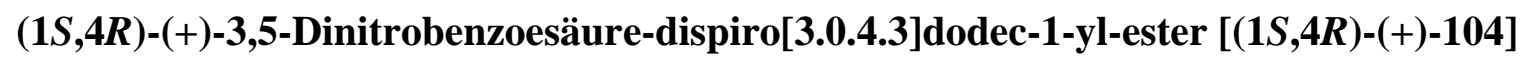

Man löste 3,5-Dinitrobenzoesäurechlorid (97 mg, $0.42 \mathrm{mmol}$ ) und (1S,4R)-(+)-60 (63 mg, $0.63 \mathrm{mmol})$ in Pyridin $(1.2 \mathrm{ml})$, verdünnte nach $21 \mathrm{~h}$ bei Raumtemperatur mit Wasser $(3 \mathrm{ml})$ und extrahierte mit Dichlormethan (2 ml). Man wusch den Extrakt mit Wasser $(2 \times 3 \mathrm{ml})$, trocknete $\left(\mathrm{MgSO}_{4}\right)$, engte ein (Badtemperatur 50\%/15 Torr) und chromatographierte den Rückstand (107 mg) an Kieselgel in Pentan/Ether 8:2 [Säule $10 \times 1 \mathrm{~cm}, \mathrm{R}_{\mathrm{f}}=0.48((1 S, 4 R)$ (+)-104)]. Man erhielt $97 \mathrm{mg}$ farblosen Feststoff. Kristallisation aus Aceton (2.0 ml) durch Diffusion von Wasser $(2.5 \mathrm{ml})$ lieferte $87 \mathrm{mg}(66 \%)$ reines $(1 S, 4 R)-(+)-104$ als farblose Kristalle vom Schmp. $81-84^{\circ} \mathrm{C}\left(99 \%\right.$ ee; $[\alpha]_{\mathrm{D}}{ }^{20}=+95.0$, c 1.19 , Aceton).

$\mathrm{C}_{19} \mathrm{H}_{22} \mathbf{N}_{2} \mathbf{O}_{6}(374.4)$

Ber.: C $60.95 \quad$ H 5.92

Gef.: C $61.00 \quad$ H 5.98

IR (Abb. 44) (KBr): $1725 \mathrm{~cm}^{-1}(\mathrm{C}=\mathrm{O})$.

${ }^{1} \mathbf{H}-\mathbf{N M R}$ (Abb. 85) (600 MHz, $\mathrm{C}_{6} \mathrm{D}_{6}, \mathrm{C}_{6} \mathrm{D}_{5} \mathrm{H}$ int): $\delta=1.12-1.18 \mathrm{ppm}$ (m, 1H), 1.19-1.25 (m, $1 \mathrm{H}), 1.29-1.45(\mathrm{~m}, 6 \mathrm{H}), 1.53-1.66(\mathrm{~m}, 7 \mathrm{H}), 1.90\left(\mathrm{~m}_{\mathrm{c}}, 1 \mathrm{H}\right), 2.22\left(\mathrm{~m}_{\mathrm{c}}, 1 \mathrm{H}\right), 2.27-2.31(\mathrm{~m}, 1 \mathrm{H})$, 5.27 (dd, $\mathrm{J}=8,8 \mathrm{~Hz}, 1 \mathrm{H}), 8.49$ (t, $\mathrm{J}=2 \mathrm{~Hz}, 1 \mathrm{H}), 8.72$ (d, $\mathrm{J}=2 \mathrm{~Hz}, 2 \mathrm{H})$.

${ }^{13}$ C-NMR (Abb. 127)(150.8 MHz, $\mathrm{C}_{6} \mathrm{D}_{6}, \mathrm{C}_{6} \mathrm{D}_{6} \mathrm{int}$ ): $\delta=20.27 \mathrm{ppm}(\mathrm{t}), 21.87(\mathrm{t}), 24.82(\mathrm{t})$, $25.02(\mathrm{t}), 25.48(\mathrm{t}), 31.83(\mathrm{t}), 33.34(\mathrm{t}), 33.60(\mathrm{t}), 36.98(\mathrm{t}), 54.26(\mathrm{~s}), 57.47(\mathrm{~s}), 72.54(\mathrm{~d})$, 121.90 (d), 128.52 (d), 133.44 (s), 148.29 (s), 161.53 (s).

MS (EI): $m / e=374\left(<1, \mathrm{M}^{+}\right), 121(100)$.

\section{(4R)-(+)-Dispiro[3.0.4.3]dodecan-1-on [(4R)-(+)-51]}

Zu einer Suspension von Pyridiniumchlorochromat (5.12 g, 23.7 mmol) in Dichlormethan (12 ml) gab man unter Argon und Rühren eine Lösung von (1S,4R)-(+)-60 (2.27 g, 12.7 mmol, Reinheit 93\%) in Dichlormethan (12 ml), wobei ein exothermer Effekt und eine Abscheidung schwarzer Zersetzungsprodukte zu beobachten war. Nach 1 h bei Raumtemperatur war die Reaktion laut DC [Pentan/Ether 8:2, $\mathrm{R}_{\mathrm{f}}=0.50$ ((4R)-51), 0.22 ((1S,4R)-(+)-60)] beendet. Die überstehende Lösung wurde abdekantiert und der schmierige Rückstand mit Ether $(3 \times 30 \mathrm{ml})$ extrahiert. Die vereinigten organischen Phasen wurden über Kieselgel (Säule $20 \times 4.5 \mathrm{~cm}$ ) filtriert und mit Ether eluiert. Die Lösungsmittel wurden abgezogen (Badtemperatur $40^{\circ} \mathrm{C} / 15$ Torr) und der Rückstand (2.16 g gelbe Flüssigkeit) wurde an Kieselgel in Pentan/Ether 95:5 chromatographiert [Säule $67 \times 4.5 \mathrm{~cm}, \mathrm{R}_{\mathrm{f}}=0.20$ ((4R)-51)]. Man erhielt $1.30 \mathrm{~g}(61 \%)(4 R)-51$ als farblose Flüssigkeit (Reinheit $99 \%$ GC, $>99 \%$ ee; $[\alpha]_{D}{ }^{20}=+145$, с 1.23, Aceton). Die chemische Reinheit wurde durch GC bestimmt [Säule A, $230^{\circ} \mathrm{C}$; Retentionszeit (min): 3.71 $((4 R)-(+)-51)]$. Die optische Reinheit folgte aus dem Drehwert $\left([\alpha]_{\mathrm{D}}{ }^{20}=+145\right.$, с 1.21 , Aceton) von aus (4S*)-51 mit (+)-(S)-N,S-Dimethyl-S-phenylsulfonimid unter anschließender 
Thermolyse erhaltenem enantiomerenreinem Material (s. oben). Die ${ }^{1} \mathrm{H}$ - und ${ }^{13} \mathrm{C}-\mathrm{NMR}-\mathrm{Daten}$ stimmten mit denen des Racemats überein.

\section{(4S)-(-)-Dispiro[3.0.4.3]dodecan-1-on [(4S)-(-)-51]}

Die Synthese wurde wie für (4R)-(+)-51 beschrieben durchgeführt. $179 \mathrm{mg}$ (1.00 mmol) $(1 S, 4 S)-(+)-62$ ergaben 91 mg (53\%) (4S)-(-)-51 (Reinheit 99\% GC, 69\% ee, $[\alpha]_{\mathrm{D}}{ }^{20}=-84.4$, $\mathrm{c}=1.24$, Aceton). Die optische Reinheit folgte aus dem Drehwert $\left([\alpha]_{\mathrm{D}}{ }^{20}=-145\right.$, с 1.14 , Aceton) von aus (4S*)-51 mit (+)-(S)-N,S-Dimethyl-S-phenylsulfonimid unter anschließender Thermolyse erhaltenem enantiomerenreinem Material (s. oben). Die ${ }^{1} \mathrm{H}$ - und ${ }^{13} \mathrm{C}-\mathrm{NMR}-\mathrm{Daten}$ stimmten mit denen des Racemats überein.

\section{(1S,4R)-(-)-1-Cyclopent-1-enyl-dispiro[3.0.4.3]dodecan-1-ol [(1S,4R)-(-)-52]}

Die Synthese wurde wie für $\left(1 R^{*}, 4 S^{*}\right)-52$ beschrieben durchgeführt. $2.19 \mathrm{~g}$ (12.3 mmol) (4R)-(+)-51 (Reinheit 99\%, >99\% ee) ergaben 2.78 g (88\%) (1S,4R)-(-)-52 (Reinheit 95\%, $>99 \%$ ee; $[\alpha]_{\mathrm{D}}{ }^{20}=-46.1$, c 1.30 , Aceton). Die ${ }^{1} \mathrm{H}$ - und ${ }^{13} \mathrm{C}-\mathrm{NMR}$ Daten waren mit denen des Racemats identisch.

\section{$(6 R, 7 S, 8 R)-(-)-8-B r o m-t r i s p i r o[4.0 .0 .4 .3 .3] h e p t a d e c a n-12-o n e ~[(6 R, 7 S, 8 R)-(-)-54]$}

Die Synthese wurde wie für $\left(6 S^{*}, 7 S^{*}, 8 R^{*}\right)-54$ beschrieben durchgeführt. $2.71 \mathrm{~g}$ (11.0 mmol) (1S,4R)-(-)-52 (Reinheit 95\%, >99\% ee) ergaben 2.55 g (75\%) (6R,7S,8R)-(-)-54 (Reinheit $99 \%,>99 \%$ ee; $[\alpha]_{\mathrm{D}}{ }^{20}=-46.3$, c 1.24 , Aceton). Die ${ }^{1} \mathrm{H}$ - und ${ }^{13} \mathrm{C}-\mathrm{NMR}$ Daten waren mit denen des Racemats identisch.

\section{(6R)-(-)-Trispiro[4.0.0.4.3.3]heptadecan-12-on [(6R)-(-)-55]}

Die Synthese wurde für (6S*)-55 beschrieben durchgeführt. $2.47 \mathrm{~g}$ (7.58 mmol) $(6 R, 7 S, 8 R)$ (-)-54 (Reinheit 99\%, >99\% ee) ergaben 1.37 g (73\%) (6R)-(-)-55 (Reinheit 99\%, >99\% ee; $[\alpha]_{\mathrm{D}}{ }^{20}=-127.0$, c 1.21 , Aceton). Die ${ }^{1} \mathrm{H}$ - und ${ }^{13} \mathrm{C}-\mathrm{NMR}$ Daten waren mit denen des Racemats identisch.

\section{(P)-(-)-Trispiro[4.0.0.4.3.3]heptadecan [(P)-(-)-8]}

Die Synthese wurde wie für rac-8 beschrieben durchgeführt. $961 \mathrm{mg}$ (3.90 mmol) (6R)-(-)-55 (Reinheit 99\%, >99\% ee) ergaben 729 mg (80\%) $(P)-(-)-8$ (Reinheit 98\%, >99\% ee; $[\alpha]_{\mathrm{D}}{ }^{20}=$ -62.6, с 1.10, $\mathrm{CHCl}_{3}$ ). Die ${ }^{1} \mathrm{H}$ - und ${ }^{13} \mathrm{C}-\mathrm{NMR}$ Daten waren mit denen des Racemats identisch. 


\section{$(1 R, 4 R)-1-\left[\left(1^{\prime}\right.\right.$-Methylselanyl)cyclobutyl)]dispiro[3.0.4.3]dodecan-1-ol [(1R,4R)-58]}

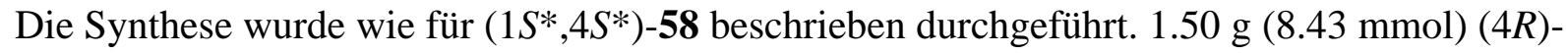
(+)-51 (Reinheit 99\%, >99\% ee) ergaben 1.56 g (40\%) (1R,4R)-58 als 70:30 Gemisch mit (4R)-(+)-51 Die ${ }^{1} \mathrm{H}$ - und ${ }^{13} \mathrm{C}-\mathrm{NMR}-\mathrm{Daten}$ waren mit denen des Gemisches der Racemate identisch.

\section{(5R)-(-)-Trispiro[3.0.0.4.3.3]hexadecan-16-one [(5R)-(-)-57]}

Die Synthese wurde wie für (5S*)-57 beschrieben durchgeführt. 1.55 g (4.74 mmol) $(1 R, 4 R)$ 58 (Reinheit 70\%) ergaben 595 mg (76\%) (5R)-(-)-57 (Reinheit 99\%, >99\% ee; $[\alpha]_{D}{ }^{20}=-$ 12.7, c 1.23, Aceton). Die ${ }^{1} \mathrm{H}$ - und ${ }^{13} \mathrm{C}-\mathrm{NMR}$ Daten waren mit denen des Racemats identisch.

\section{(5R)-(-)-Trispiro[3.0.0.4.3.3]hexadecane [(5R)-(-)-15]}

Die Synthese wurde wie für (5S*)-15 beschrieben durchgeführt. $464 \mathrm{mg}$ (2.00 mmol) (5R)(-)-57 (Reinheit 99\%, >99\% ee) ergaben 287 mg (66\%) (5R)-(-)-15 (Reinheit 98\%, >99\% ee; $[\alpha]_{\mathrm{D}}{ }^{20}=-30.7$, с $\left.1.15, \mathrm{CHCl}_{3}\right)$. Die ${ }^{1} \mathrm{H}$ - und ${ }^{13} \mathrm{C}$-NMR Daten waren mit denen des Racemats identisch. 


\section{SPEKTRENANHANG}

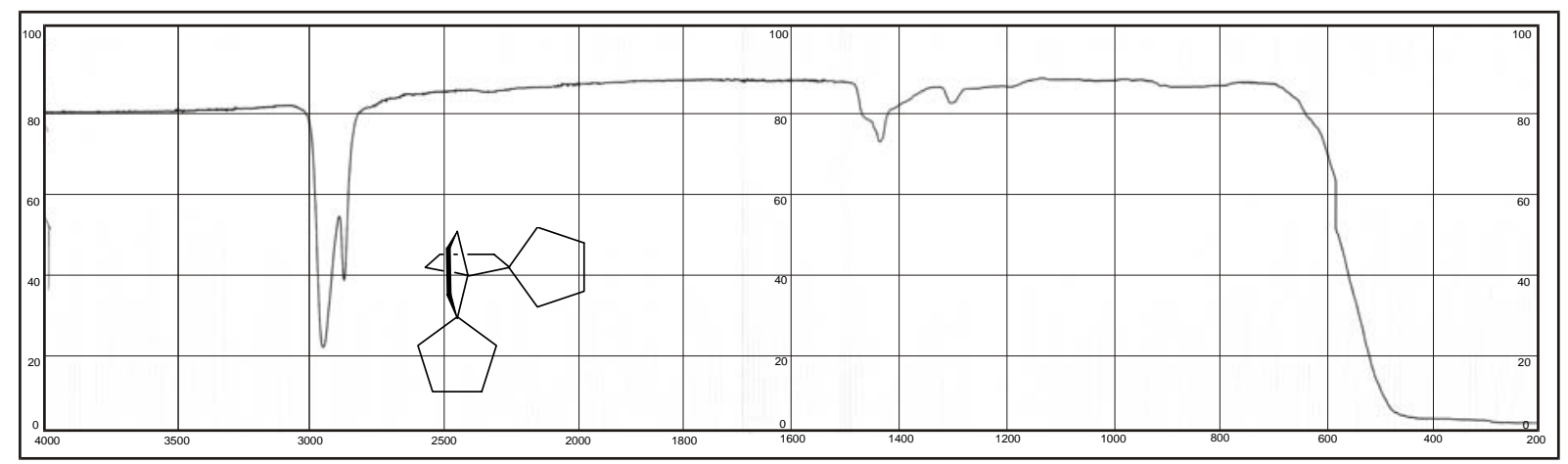

Abb. 8 IR-Spektrum von rac-Trispiro[4.0.0.4.3.3]heptadecan (rac-8)

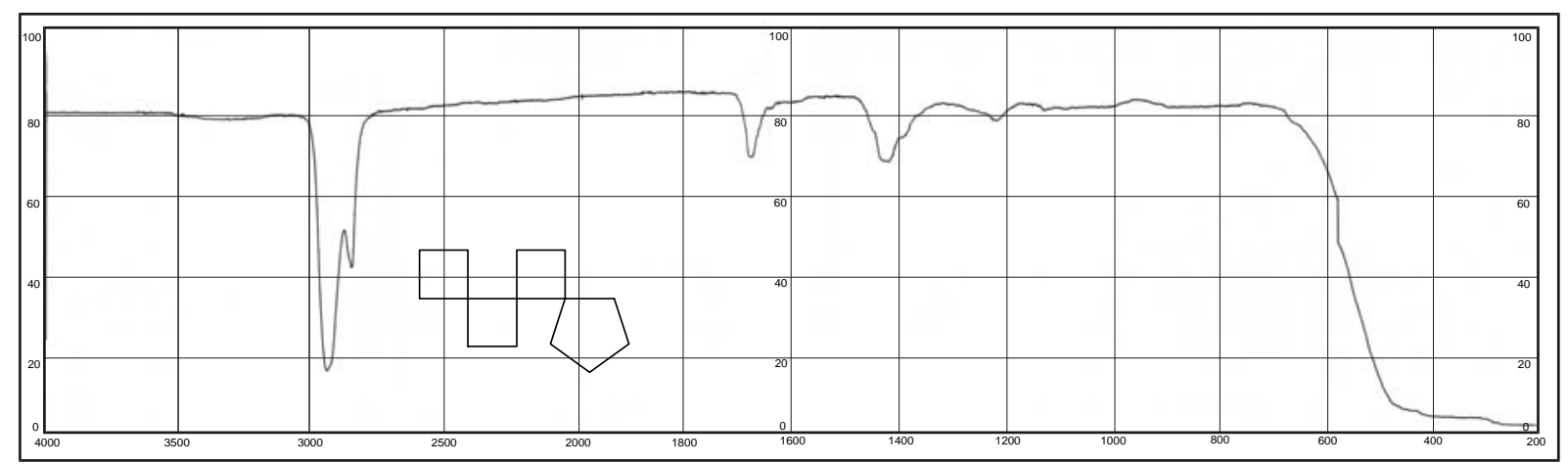

Abb. 9 IR-Spektrum von (5R*)-Trispiro[3.0.0.4.2.2]tetradecan (13)

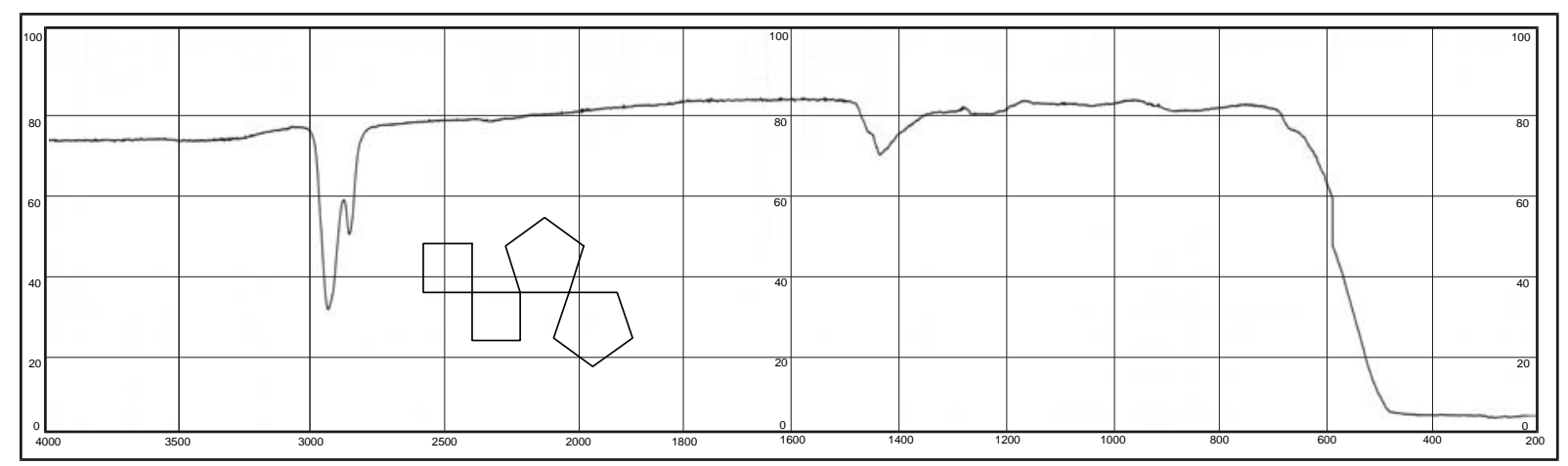

Abb. 10 IR-Spektrum von (5R*)-Trispiro[3.0.0.4.3.2]pentadecan (14) 


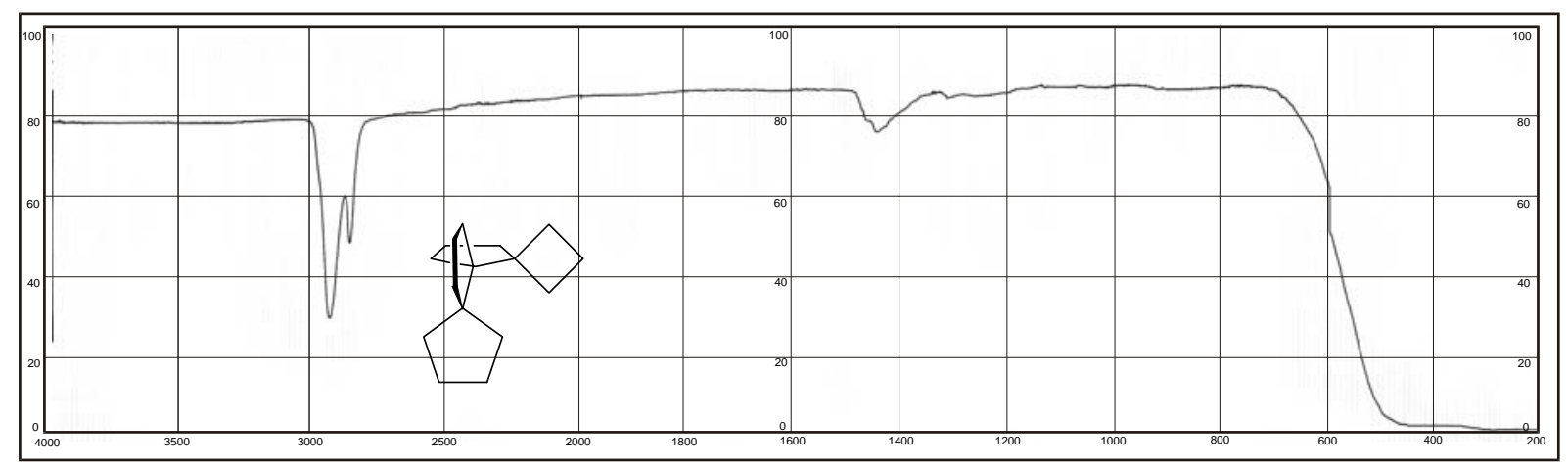

Abb. 11 IR-Spektrum von (5S*)-Trispiro[3.0.0.4.3.3]hexadecan [(5S*)-15]

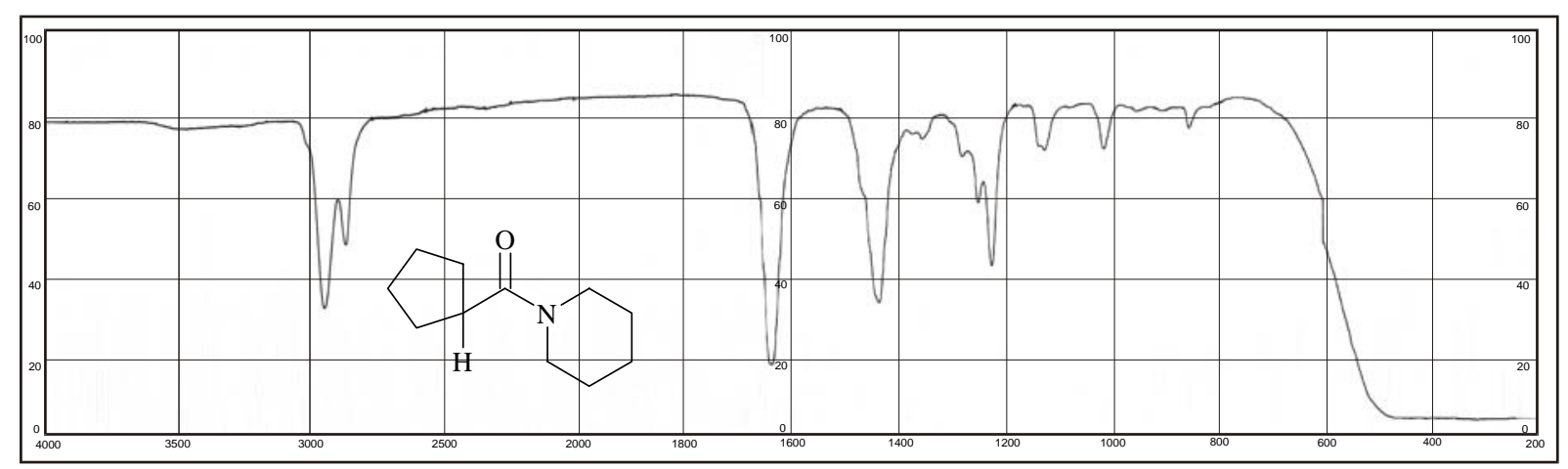

Abb. 12 IR-Spektrum von 1-(Cyclopentylcarbonyl)-piperidin (23)

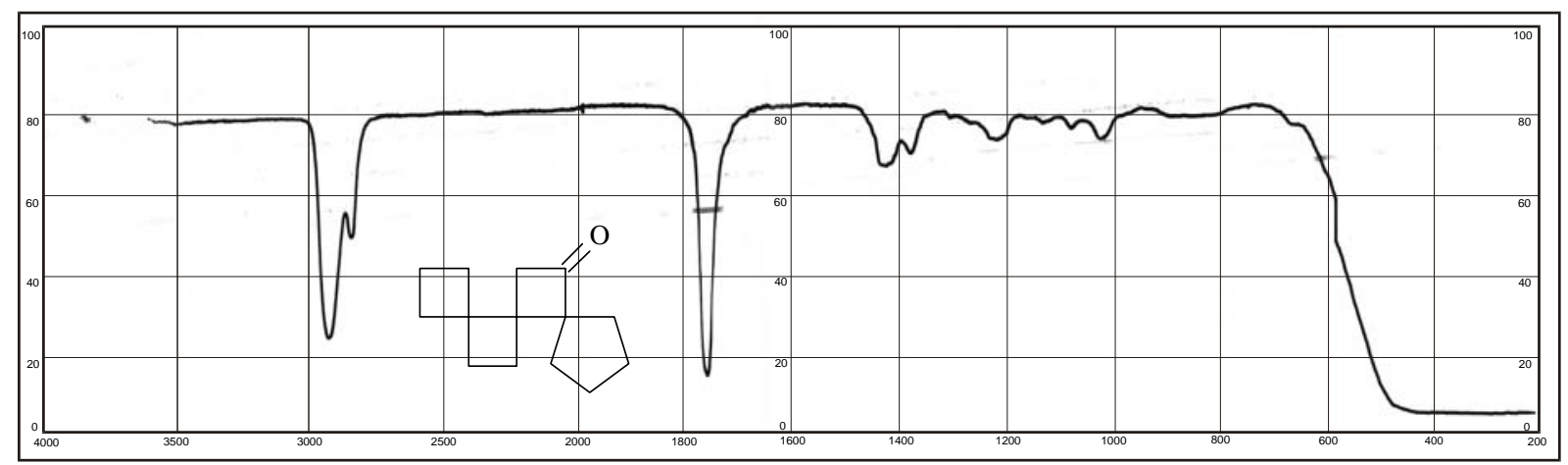

Abb. 13 IR-Spektrum von (5R*)-Trispiro[3.0.0.4.2.2]tetradecan-11-on (24) 


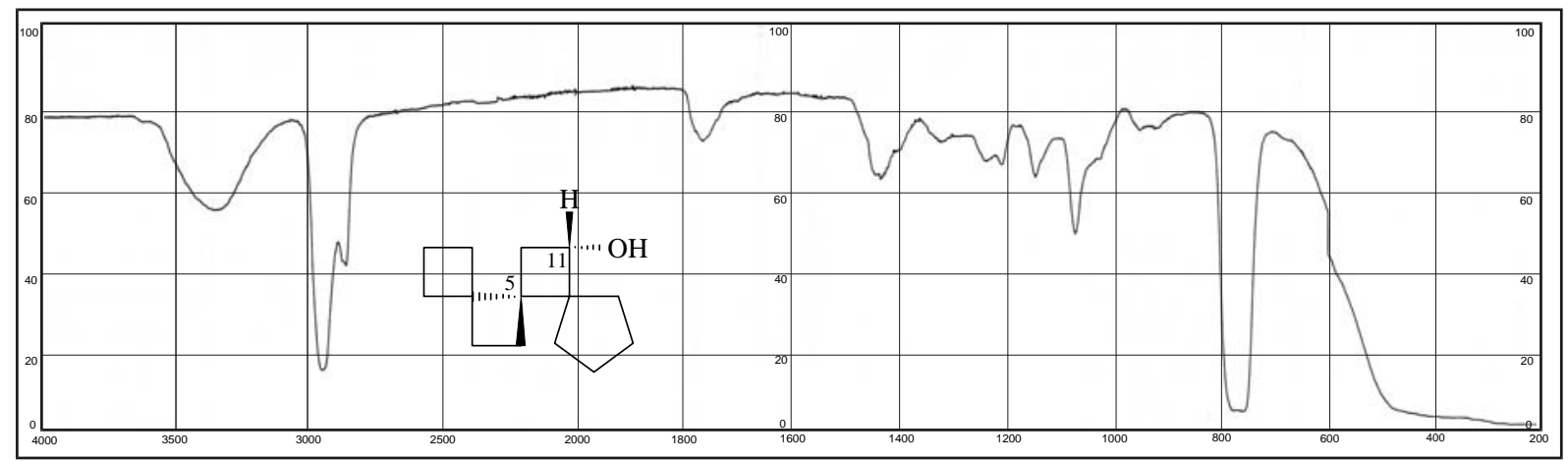

Abb. 14 IR-Spektrum von $(5 S, 11 S)-(+)$-Trispiro[3.0.0.4.2.2]tetradecan-11-ol [(5S,11S)$(+)-28)]$

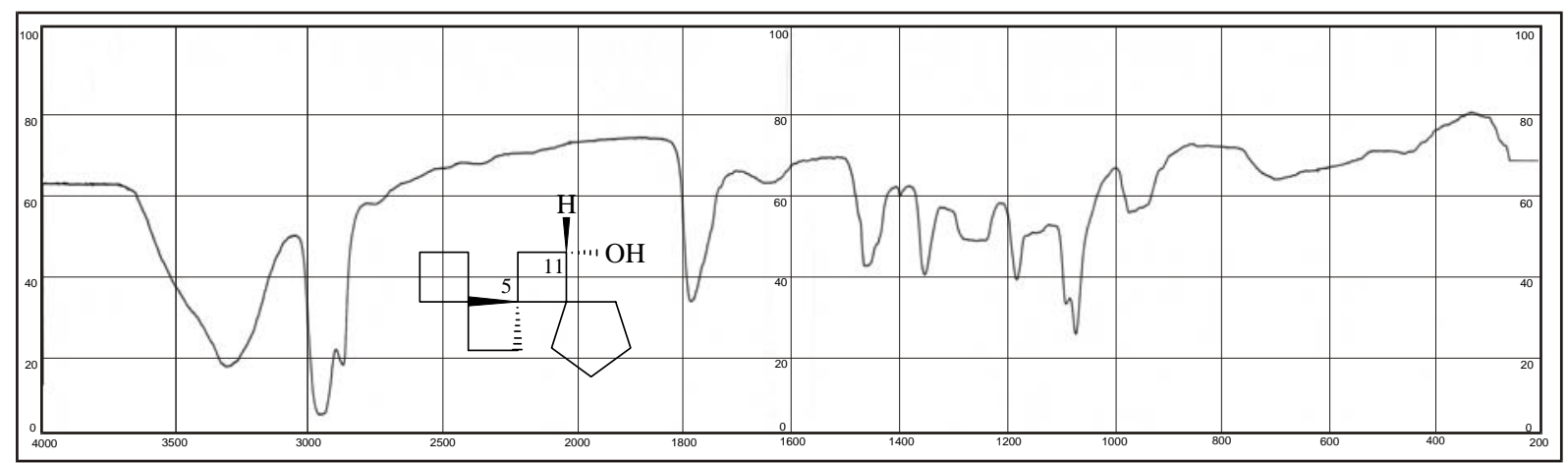

Abb. 15 IR-Spektrum von $(5 R, 11 S)-(+)-T r i s p i r o[3.0 .0 .4 .2 .2]$ tetradecan-11-ol [(5R,11S)$(+)-29)]$

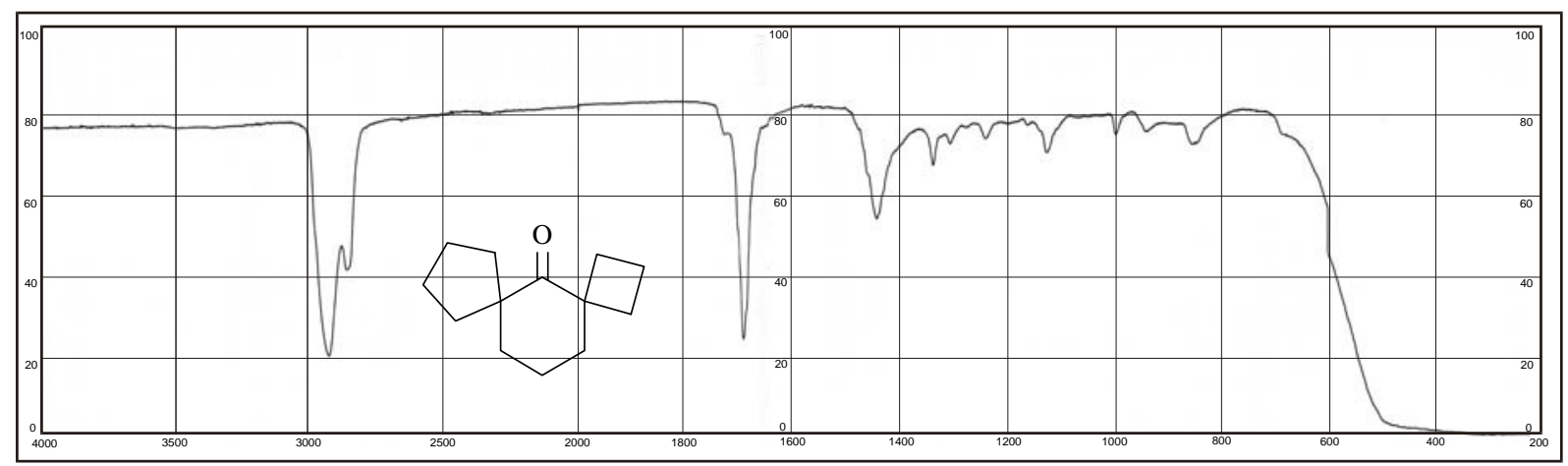

Abb. 16 IR-Spektrum von Dispiro[3.1.4.3]tridecan-5-on (31) 


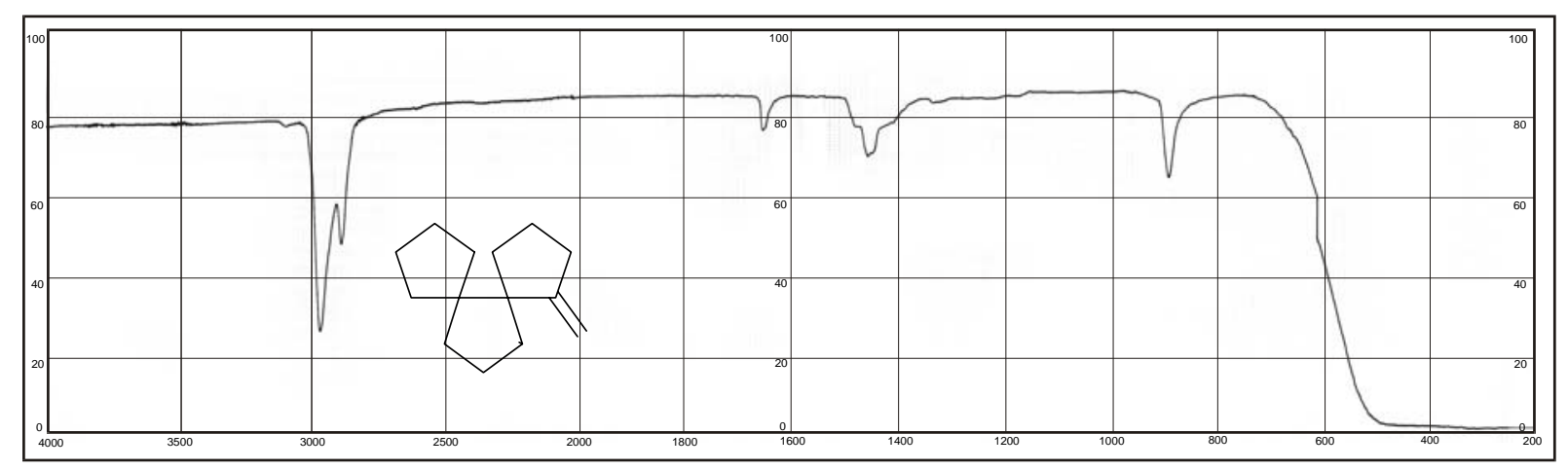

Abb. 17 IR-Spektrum von 1-Methylen-dispiro[4.0.4.3]tridecan (33)

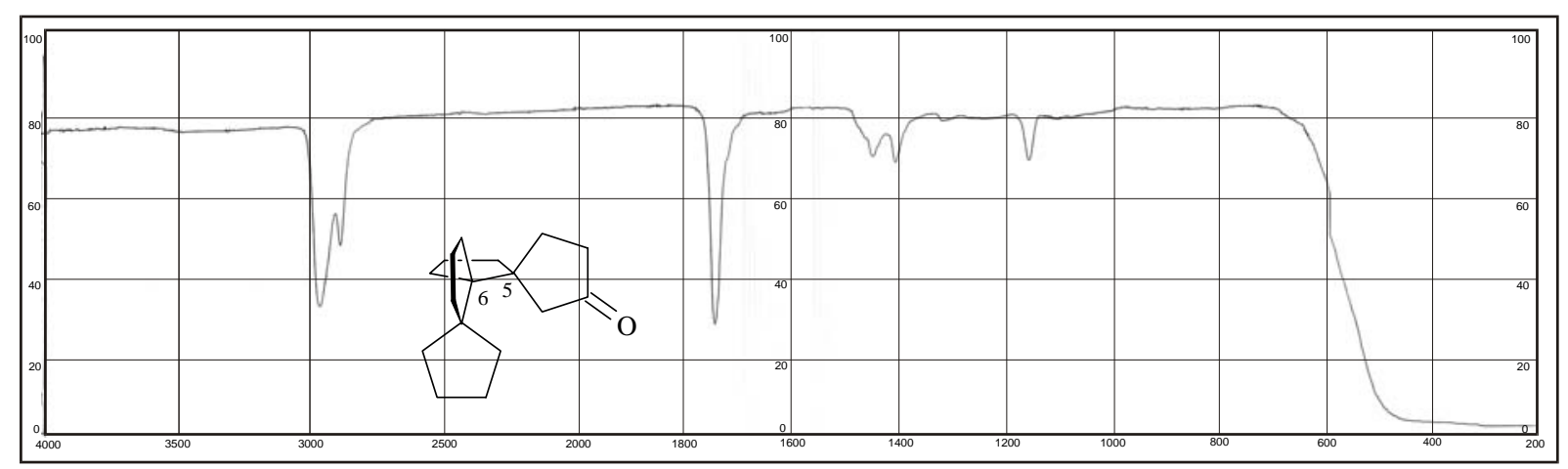

Abb. 18 IR-Spektrum von $\left(5 S^{*}, 6 S^{*}\right)$-Trispiro[4.0.0.4.3.3]heptadecan-2-on [(5S*,6S*)35]

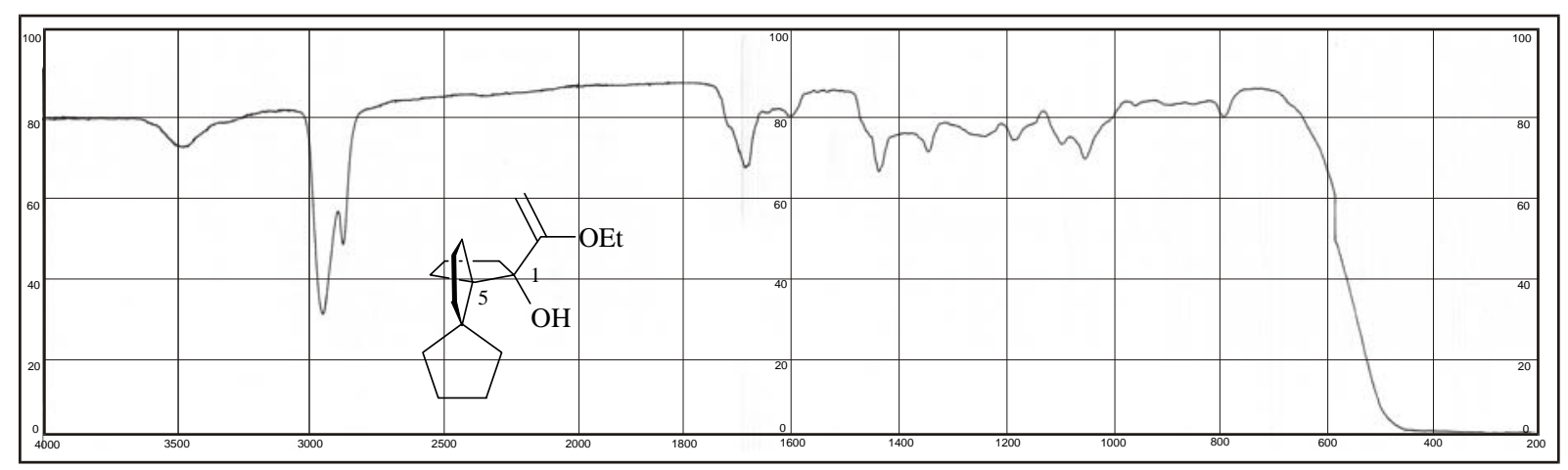

Abb. 19 IR-Spektrum von (1S*,5S*)-1-(1-Ethoxy-vinyl)-dispiro[4.0.4.3]tridecan-1-ol $\left[\left(1 S^{*}, 5 S^{*}\right)-\mathbf{4 1}\right]$ 


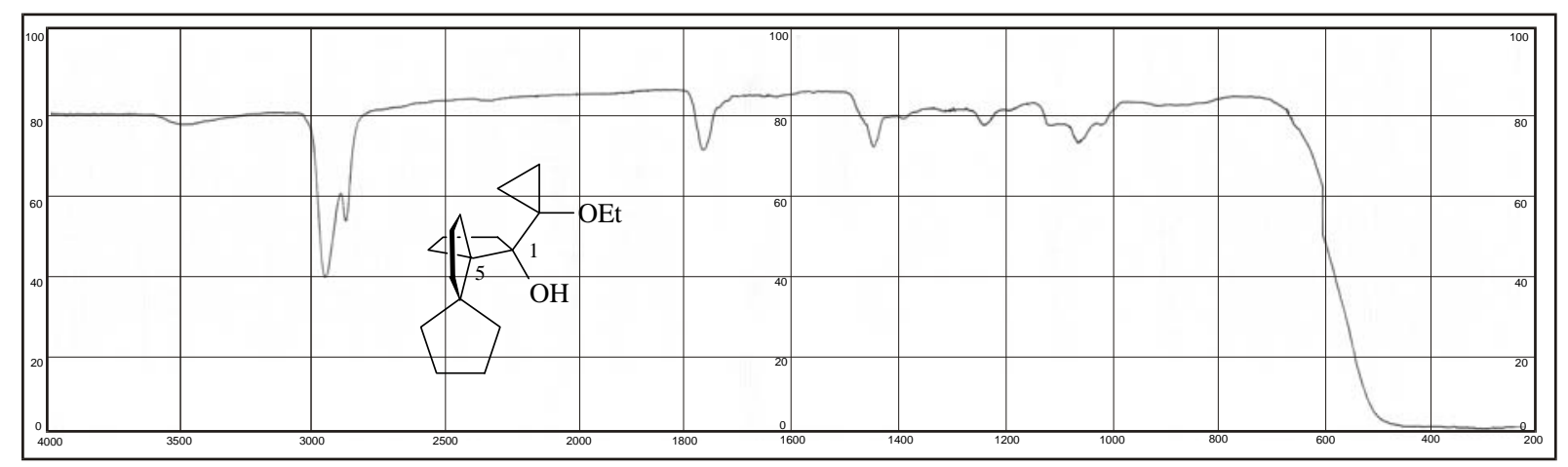

Abb. 20 IR-Spektrum von (1S*,5S*)-1-(1-Ethoxy-cyclopropyl)-dispiro[4.0.4.3]tridecan$1-\mathrm{ol}\left[\left(1 S^{*}, 5 S^{*}\right)-42\right]$

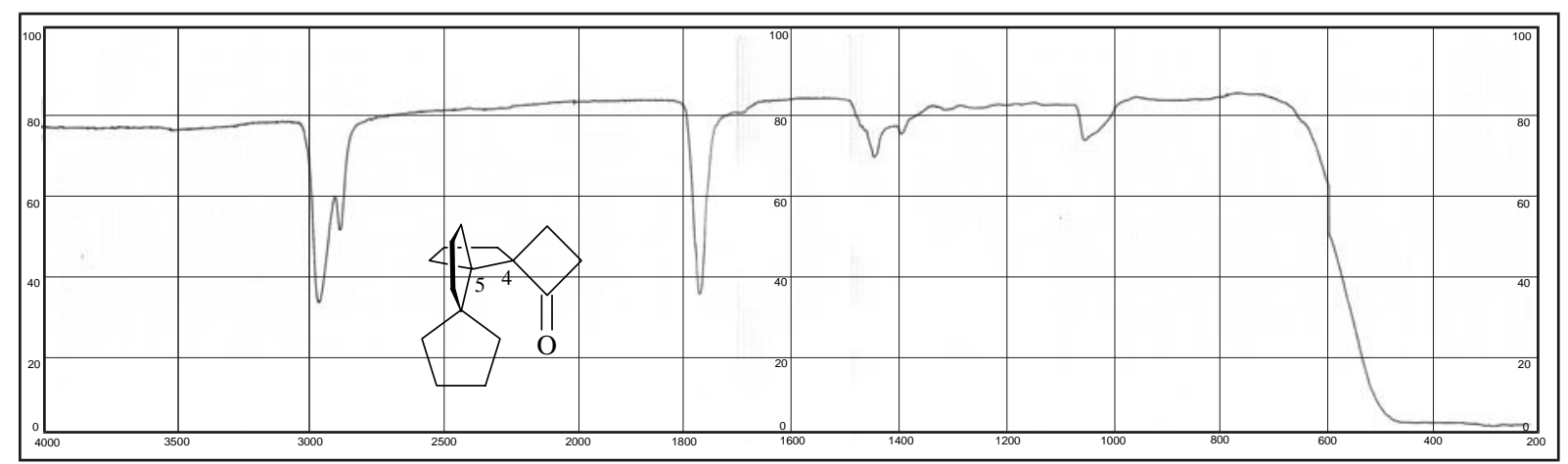

Abb. 21 IR-Spektrum von $\left(4 R^{*}, 5 S^{*}\right)$-Trispiro[3.0.0.4.3.3]hexadecan-1-on [(4R*,5S*)43]

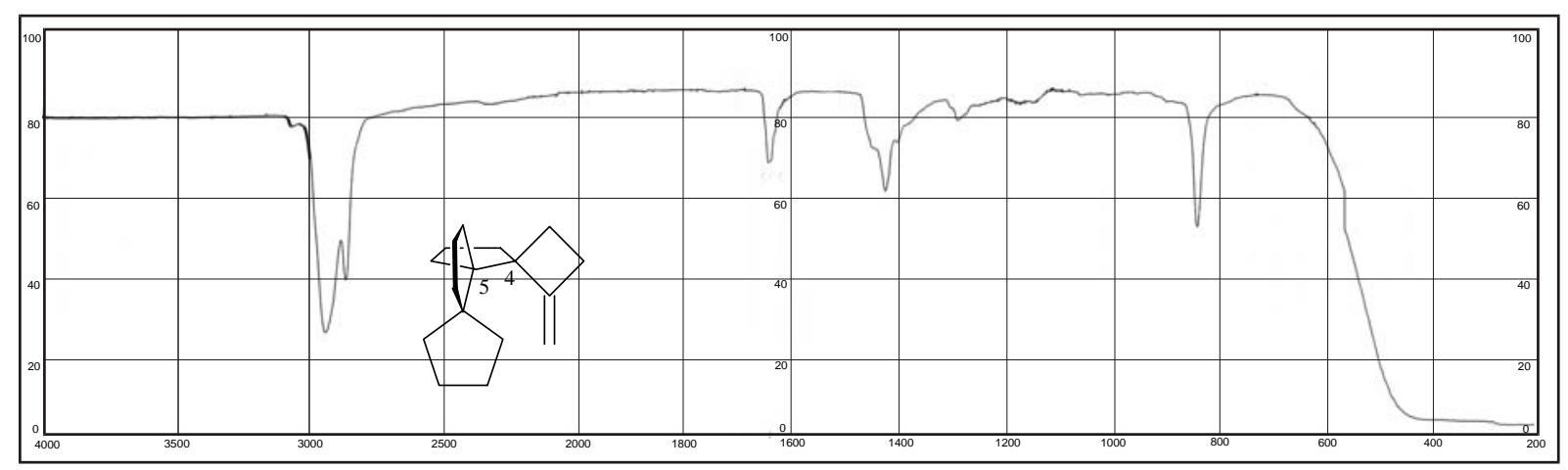

Abb. 22 IR-Spektrum von (4R*,5S*)-1-Methylen-trispiro[3.0.0.4.3.3]hexadecan $\left[\left(4 R^{*}, 5 S^{*}\right)-44\right]$ 


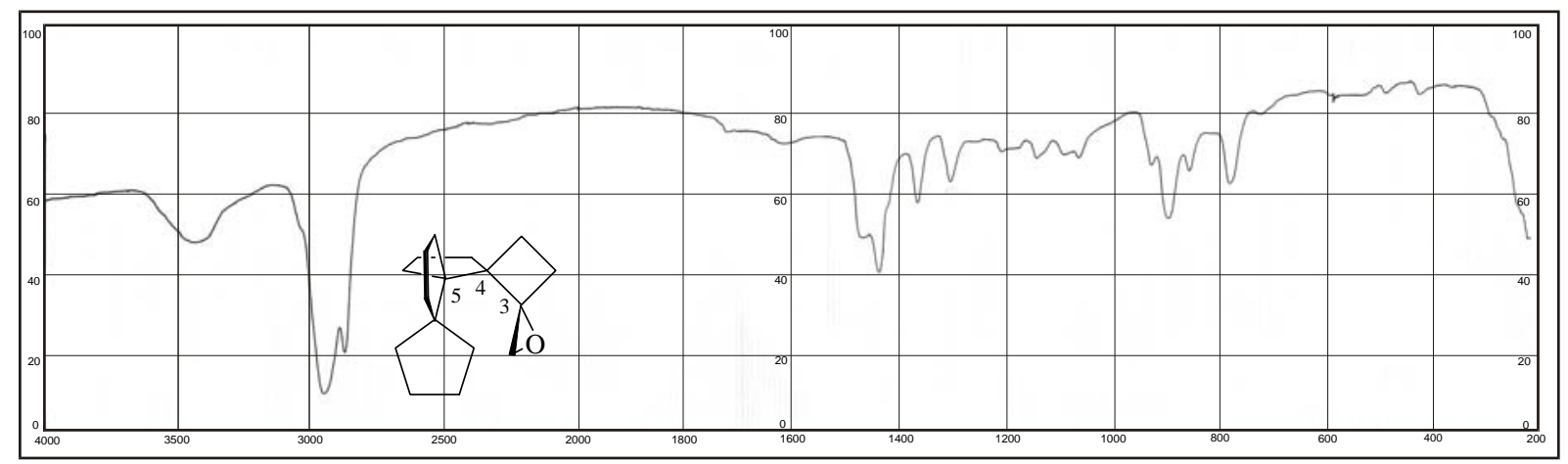

Abb. 23 IR-Spektrum von $\left(3 S^{*}, 4 R^{*}, 5 S^{*}\right)-1-O x a-t e t r a s p i r o[2.0 .0 .0 .4 .3 .3 .2]$ octadecan [(3S*,4R*,5S*)-46]

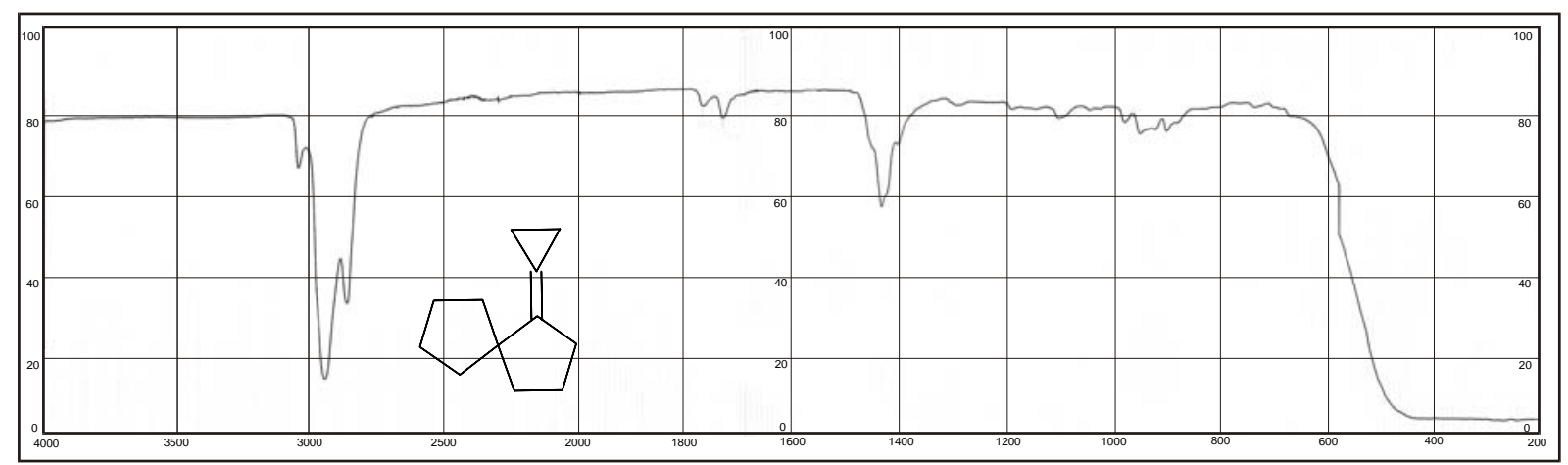

Abb. 24 IR-Spektrum von Cyclopropyliden-spiro[4.4]nonan (49)

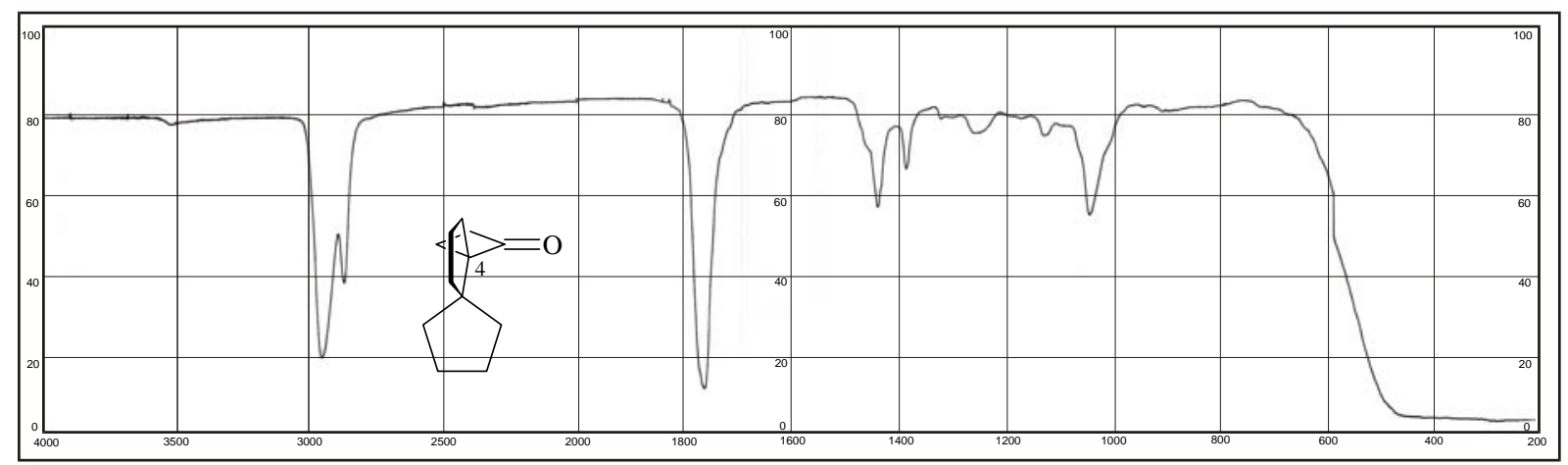

Abb. 25 IR-Spektrum von (4S*)-Dispiro[3.0.4.3]dodecan-1-on [(4S*)-51] 


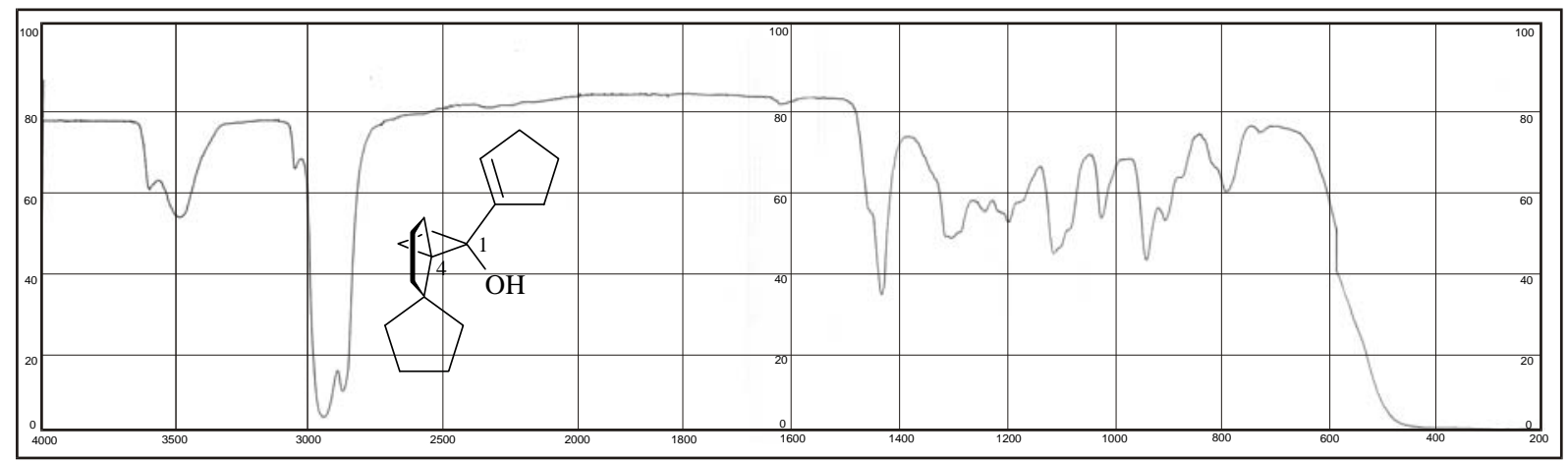

Abb. 26 IR-Spektrum von $\left(1 R^{*}, 4 S^{*}\right)$-1-Cyclopent-1-enyl-dispiro[3.0.4.3]dodecan-1-ol $\left[\left(1 R^{*}, 4 S^{*}\right)-52\right]$

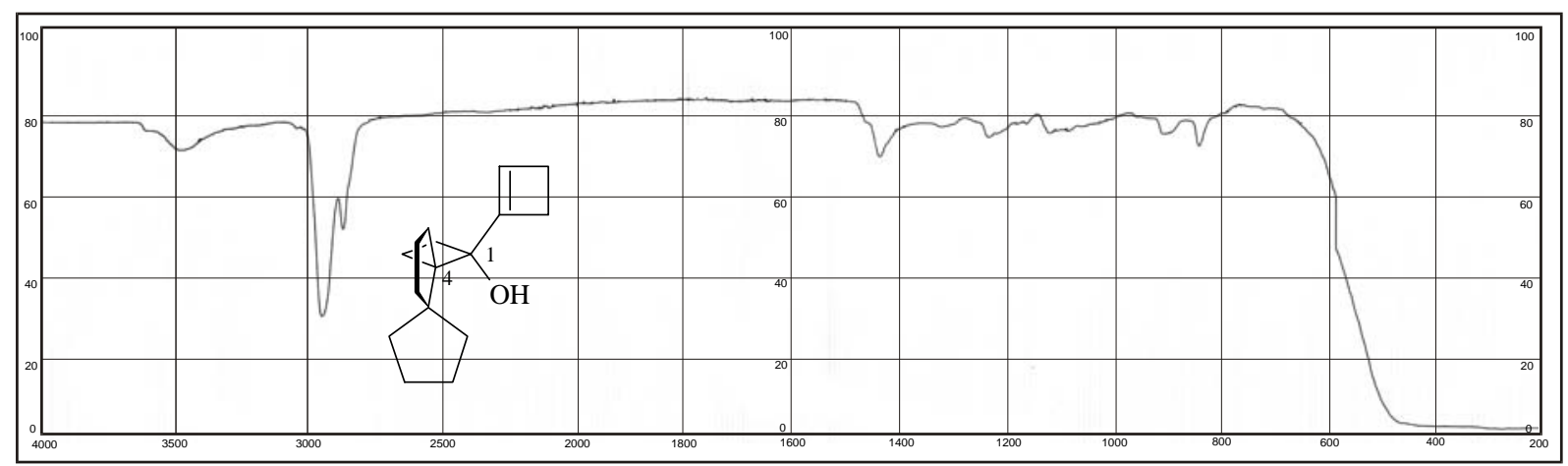

Abb. 27 IR-Spektrum von $\left(1 R^{*}, 4 S^{*}\right)$-1-Cyclobut-1-enyl-dispiro[3.0.4.3]dodecan-1-ol $\left[\left(1 R^{*}, 4 S^{*}\right)-53\right]$

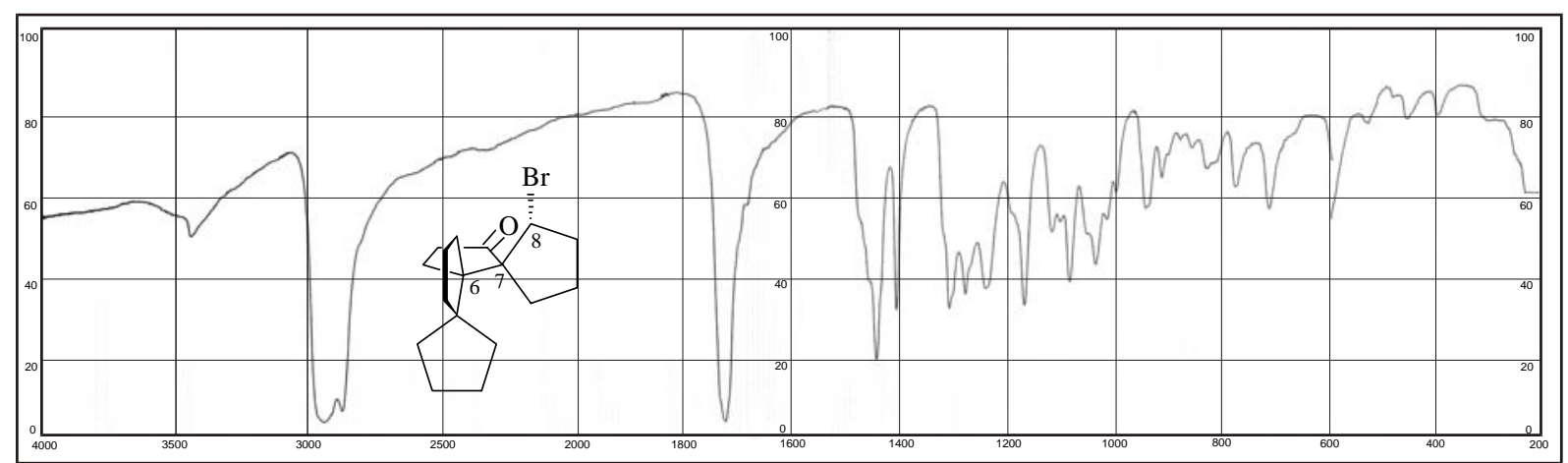

Abb. 28 IR-Spektrum von $\left(6 S^{*}, 7 S^{*}, 8 R^{*}\right)$-8-Brom-trispiro[4.0.0.4.3.3]heptadecan-12-on $\left[\left(6 S^{*}, 7 S^{*}, 8 R^{*}\right)-54\right]$ 


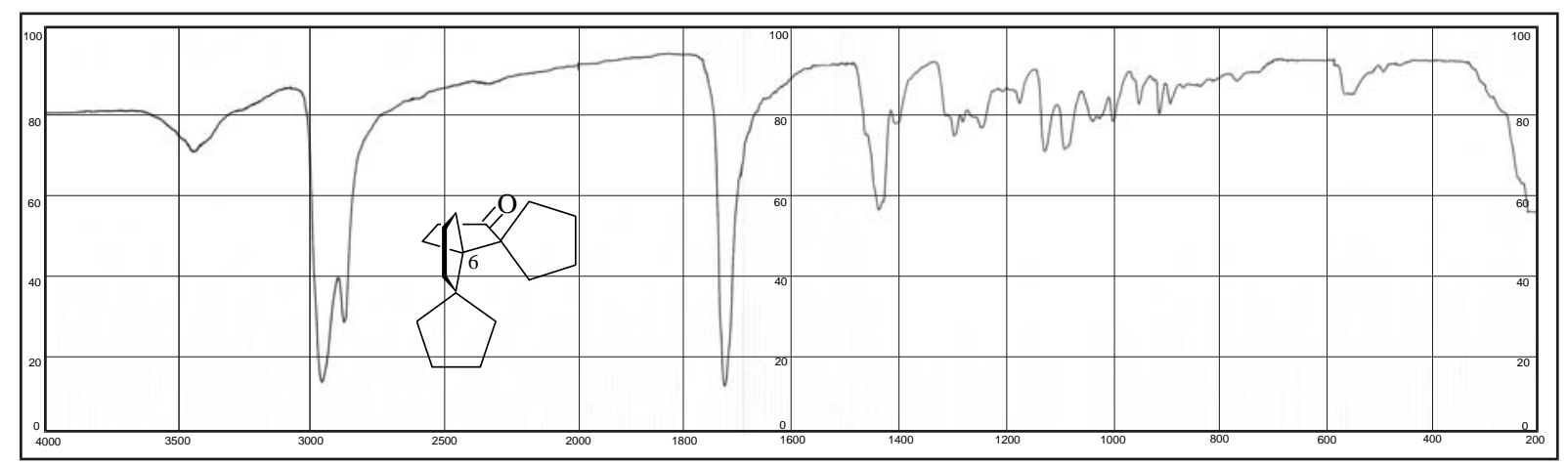

Abb. 29 IR-Spektrum von (6S*)-Trispiro[4.0.0.4.3.3]heptadecan-12-on [(6S*)-55]

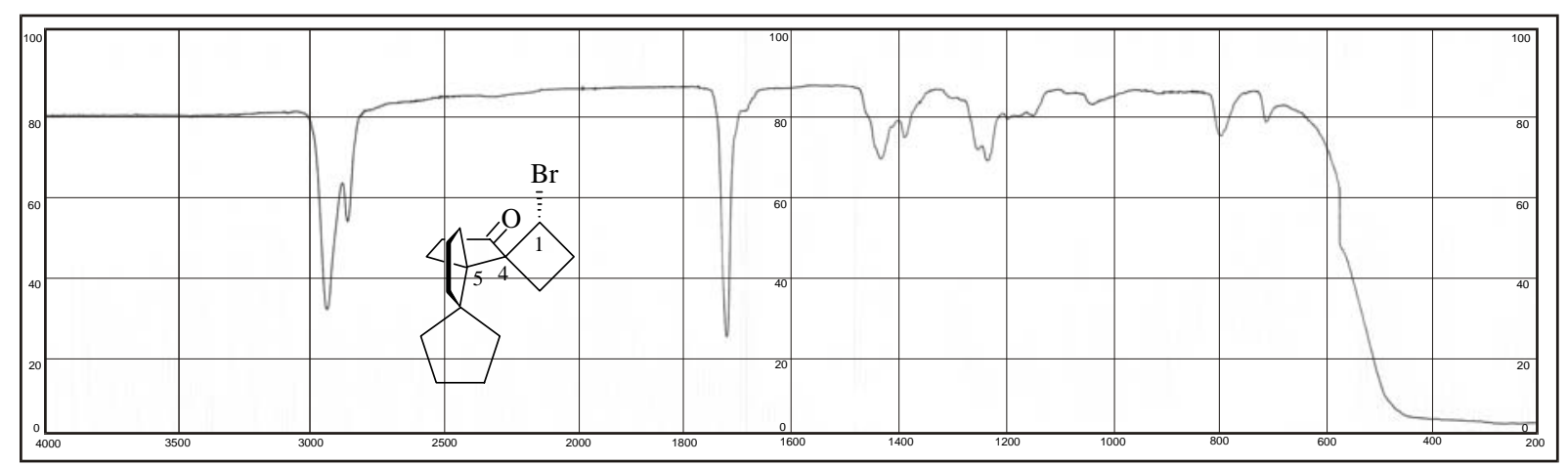

Abb. 30 IR-Spektrum von $\left(1 R^{*}, 4 S^{*}, 5 S^{*}\right)-1-B r o m-t r i s p i r o[3.0 .0 .4 .3 .3]$ hexadecan-16-on $\left[\left(1 R^{*}, 4 S^{*}, 5 S^{*}\right)-56\right]$

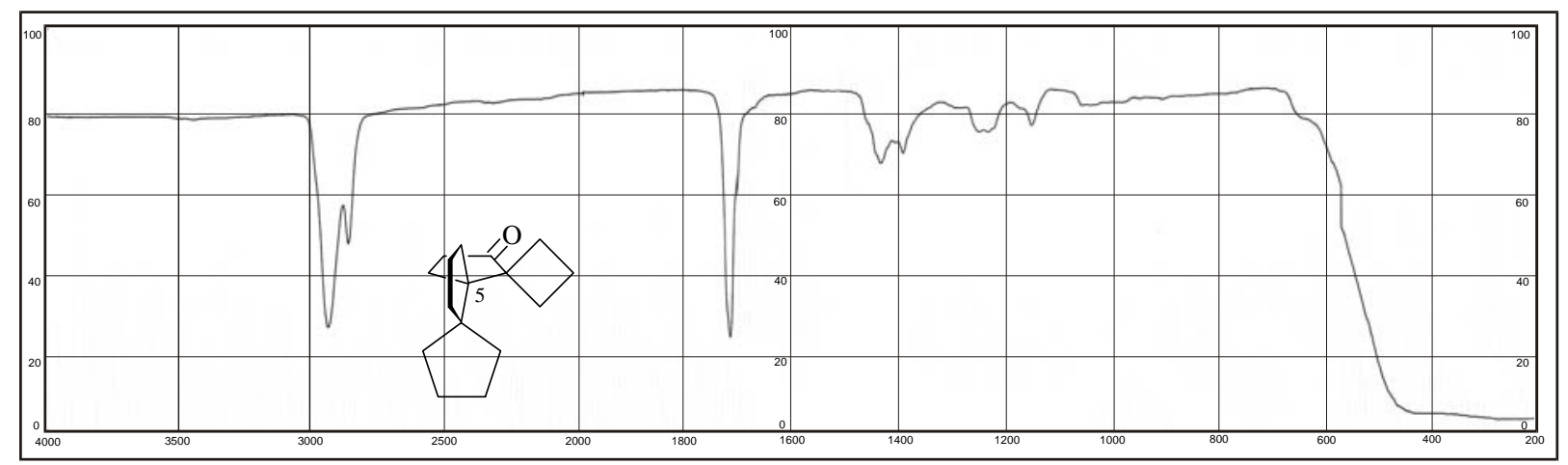

Abb. 31 IR-Spektrum von (5S*)-Trispiro[3.0.0.4.3.3]hexadecan-16-on [(5S*)-57] 


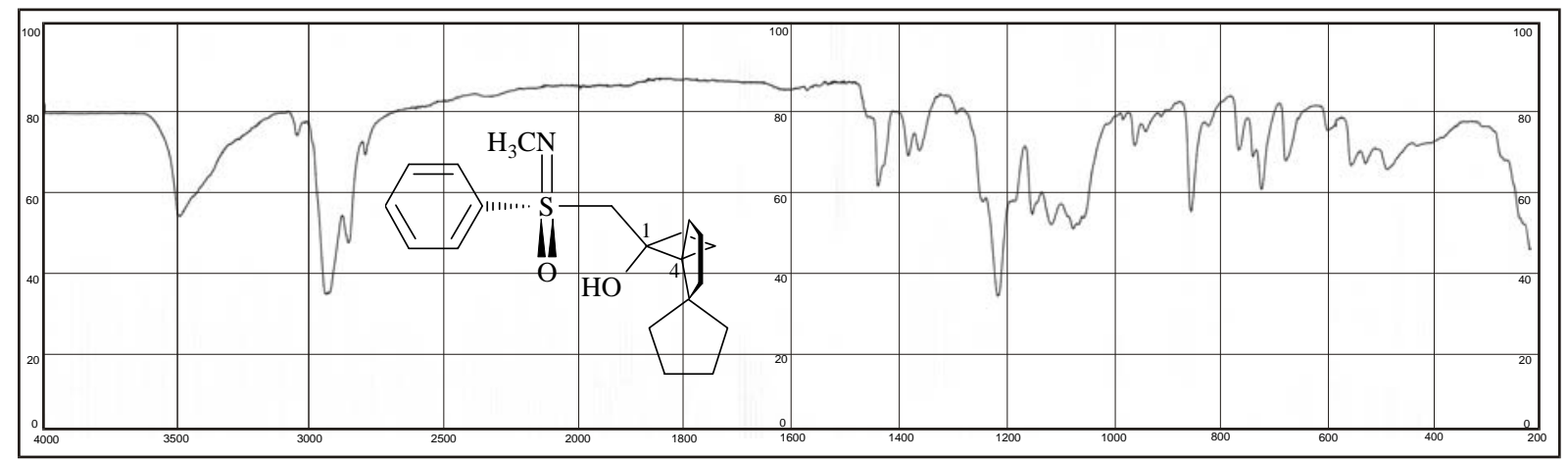

Abb. 32 IR-Spektrum von (SS,1R,4R)-(+)-[(N-Methyl-S-phenylsulfonimidoyl)methyl]dispiro[3.0.4.3]dodecan-1-ol [(SS,1R,4R)-(+)-59]

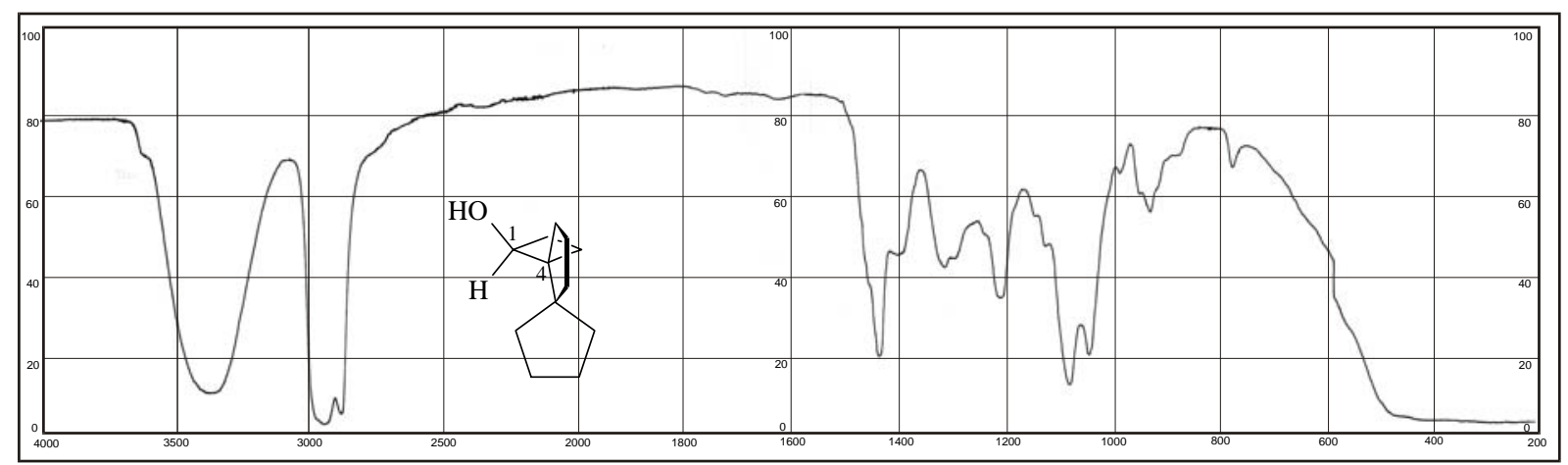

Abb. 33 IR-Spektrum von (1S,4R)-Dispiro[3.0.4.3]dodecan-1-ol [(1S,4R)-(+)-60]

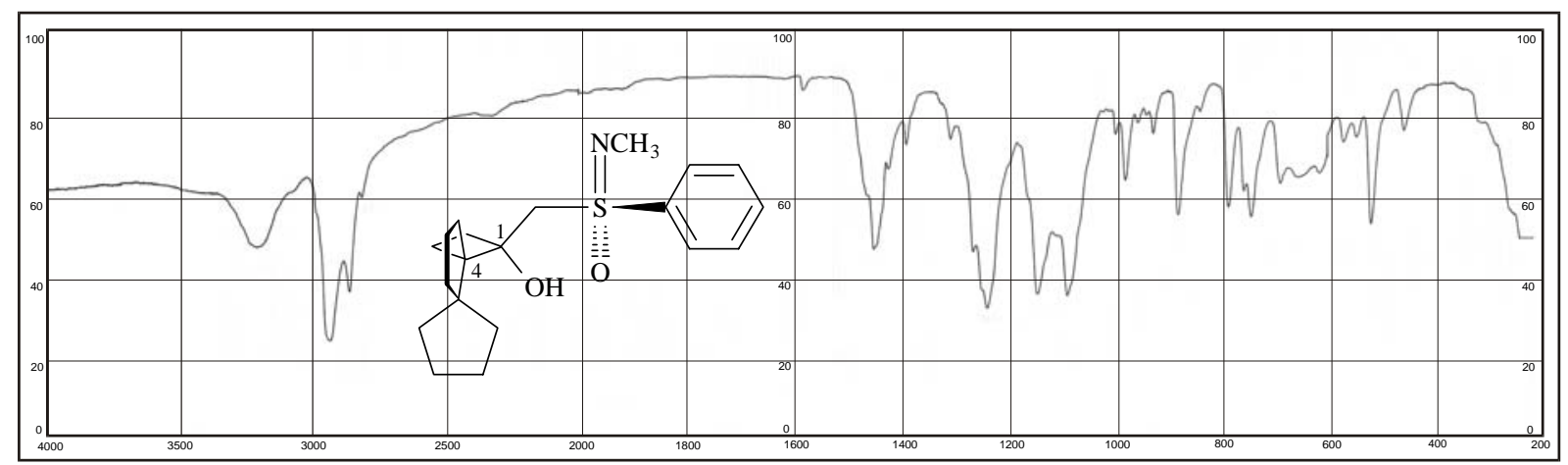

Abb. 34 IR-Spektrum von (SS,1S,4S)-(+)-[(N-Methyl-S-phenylsulfonimidoyl)methyl]dispiro[3.0.4.3]dodecan-1-ol [(SS,1S,4S)-(+)-61] 


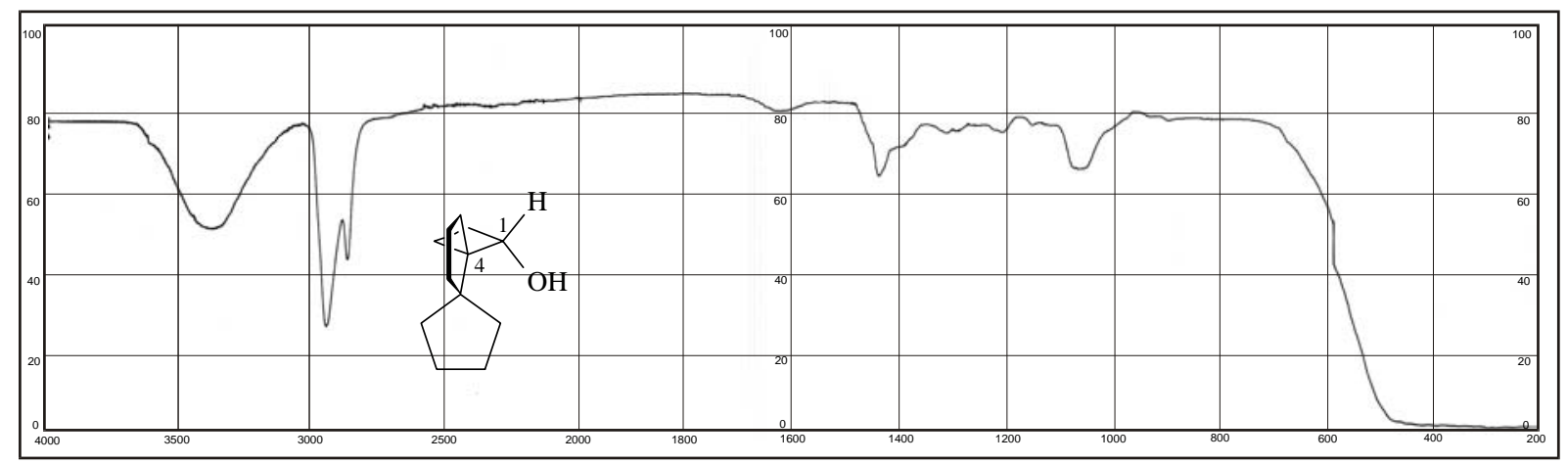

Abb. 35 IR-Spektrum von (1S,4S)-Dispiro[3.0.4.3]dodecan-1-ol [(1S,4S)-(+)-62]

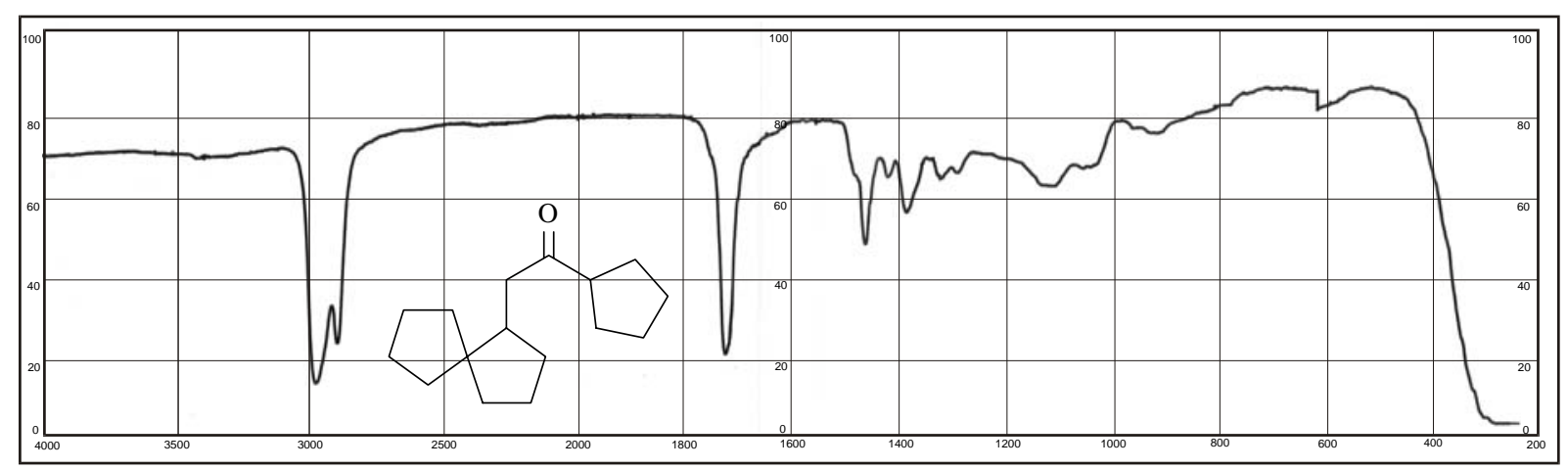

Abb. 36 IR-Spektrum von 1-Cyclopentyl-2-spiro[4.4]non-1-yl-ethanon (75)

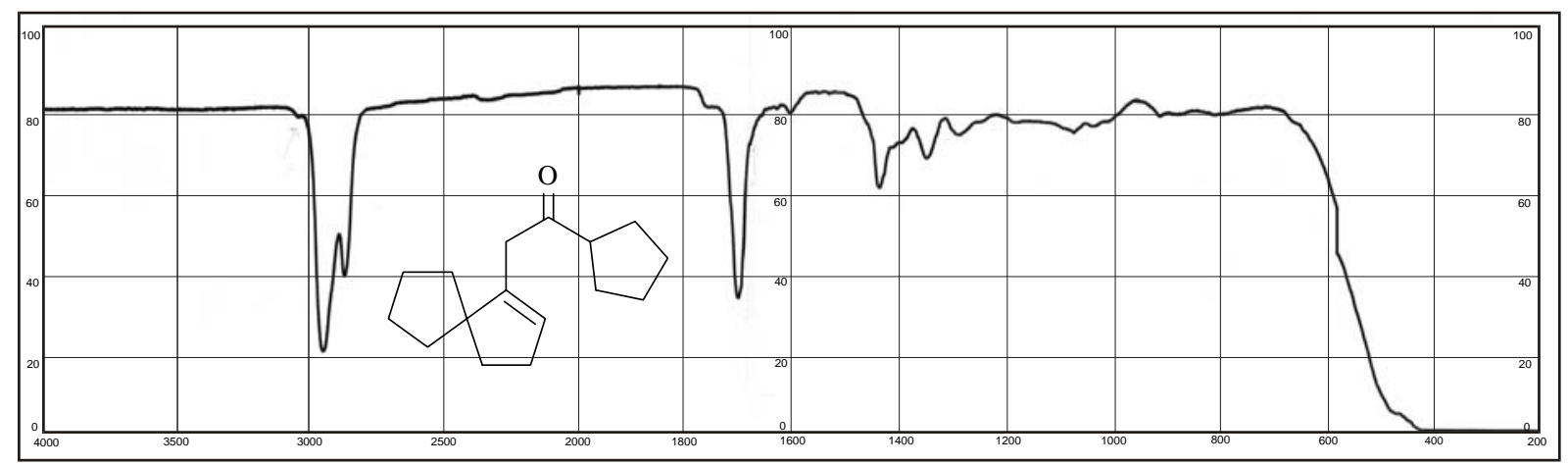

Abb. 37 IR-Spektrum von 1-Cyclopentyl-2-spiro[4.4]non-1-en-1-yl-ethanon (76) 


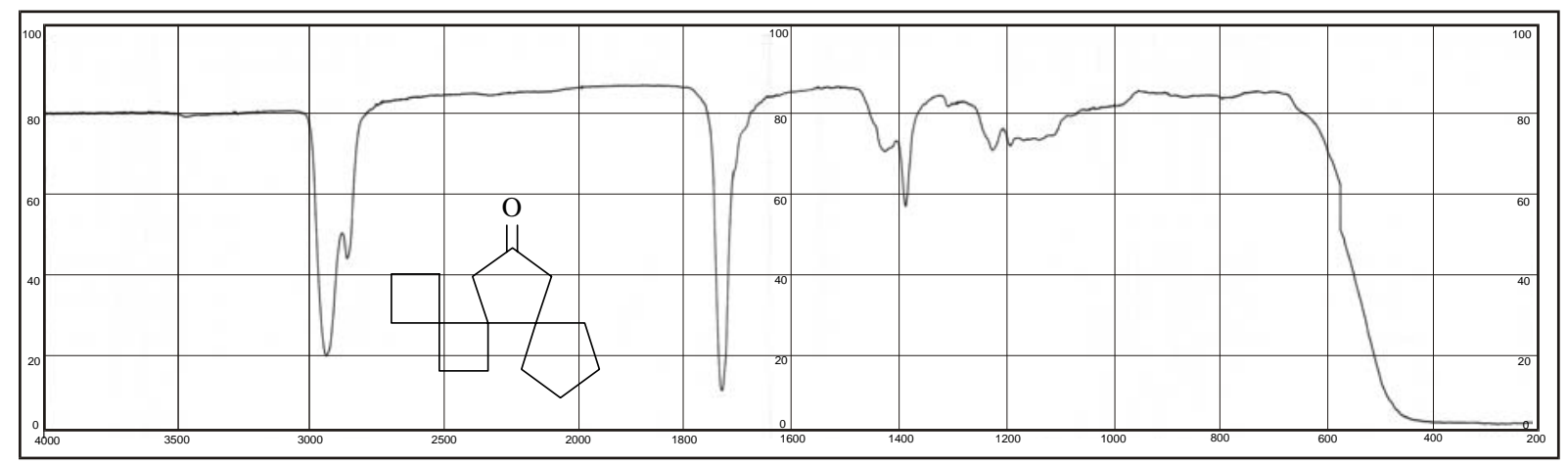

Abb. 38 IR-Spektrum von (5R*)-Trispiro[3.0.0.4.3.2]tetradecan-12-on (77)

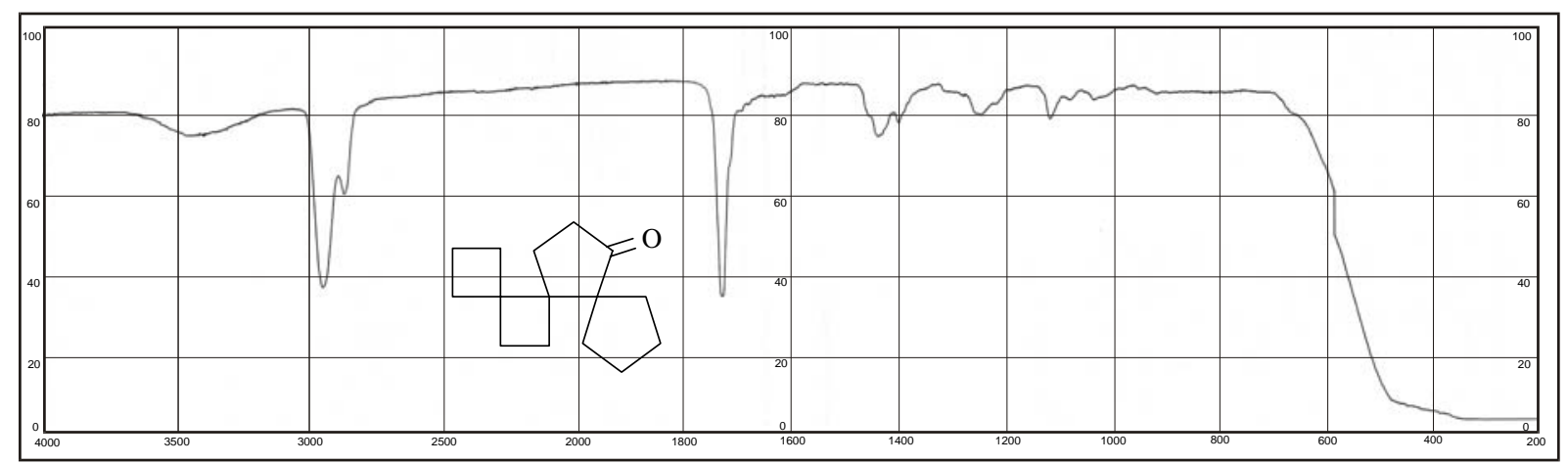

Abb. 39 IR-Spektrum von (5R*)-Trispiro[3.0.0.4.3.2]tetradecan-11-on (78)

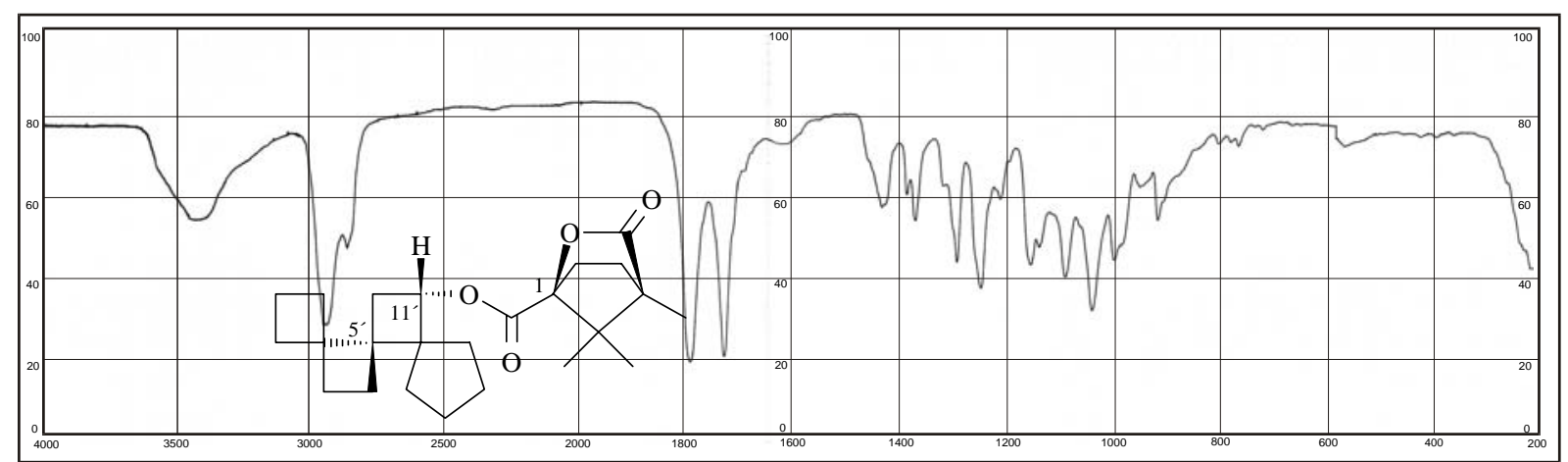

Abb. 40 IR-Spektrum von (1S)-(+)-4,7,7-Trimethyl-3-oxo-2-oxa-bicyclo[2.2.1]heptan1-carbonsäure(5'S,11'S)-trispiro[3.0.0.4.2.2]tetradecan-11-yl-ester $\left[\left(1 S, 5^{\prime} S, 11^{\prime} S\right)-(+)-79\right]$ 


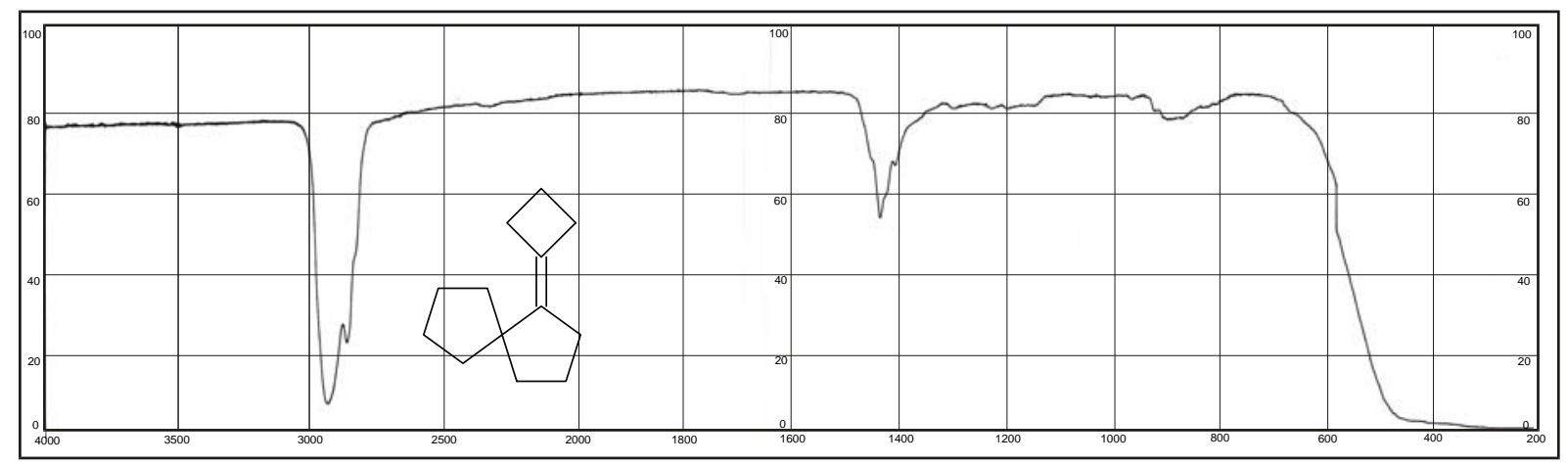

Abb. 41 IR-Spektrum von Cyclobutyliden-spiro[4.4]nonan (85)

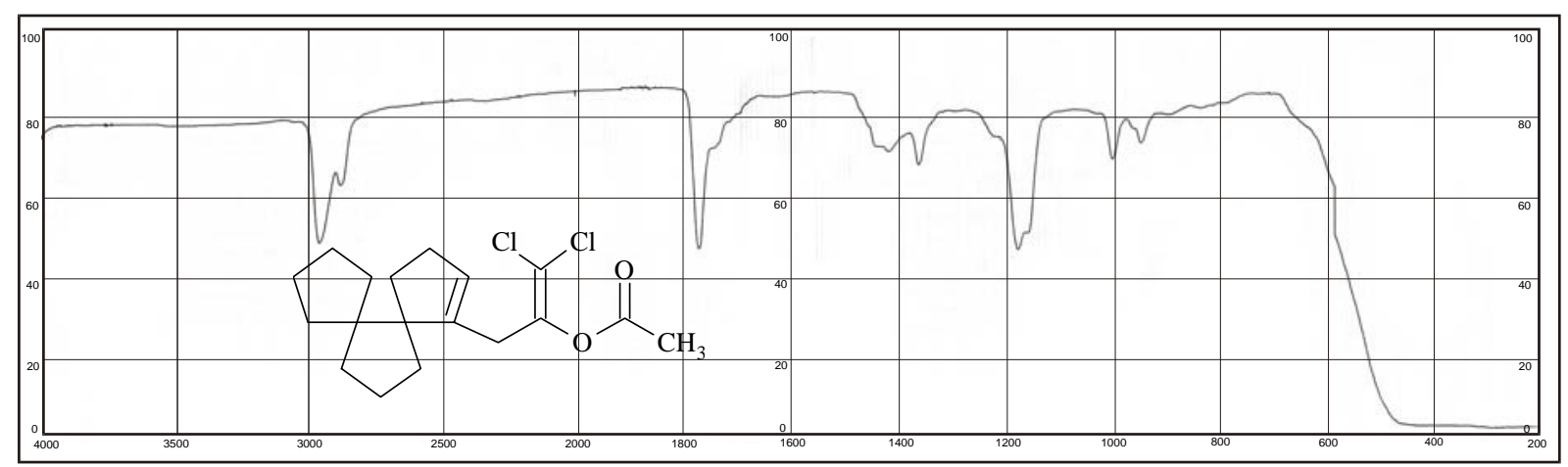

Abb. 42 IR-Spektrum von Essigsäure-2,2-dichlor-1-dispiro[4.0.4.3]tridec-1-en-1-ylmethyl-vinyl-ester (95)

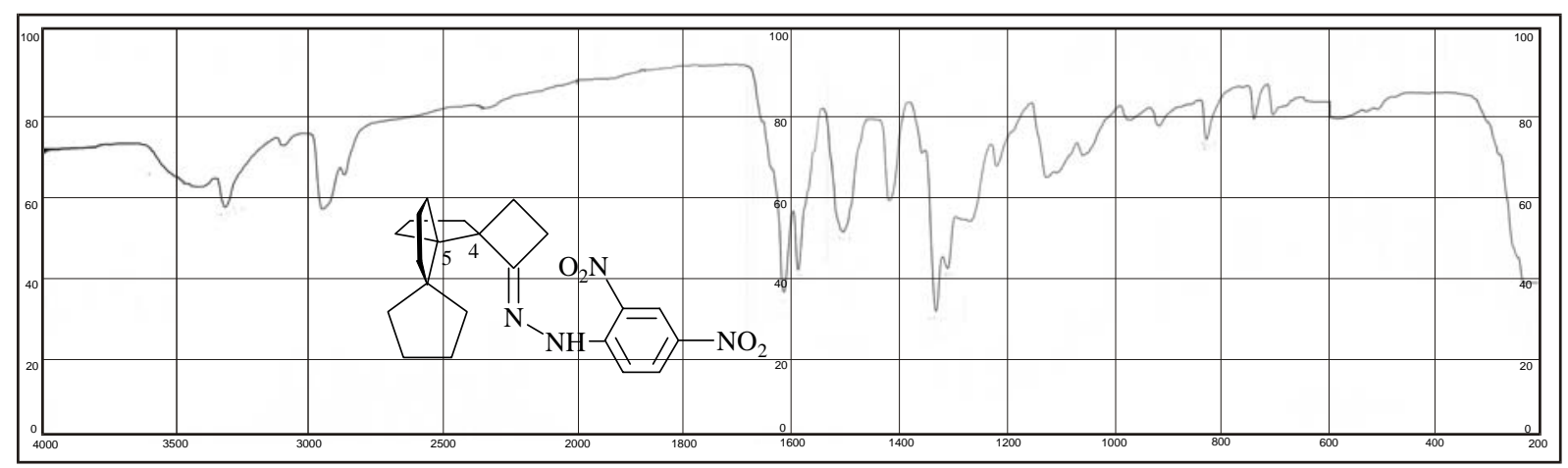

Abb. 43 IR-Spektrum von $\left(4 R^{*}, 5 S^{*}\right)-\mathrm{N}-$ Trispiro[3.0.0.4.3.3]hexadec-1-yliden- $\mathrm{N}^{\prime}-2,4-$ dinitrophenyl-hydrazin [(4R*,5S*)-98] 


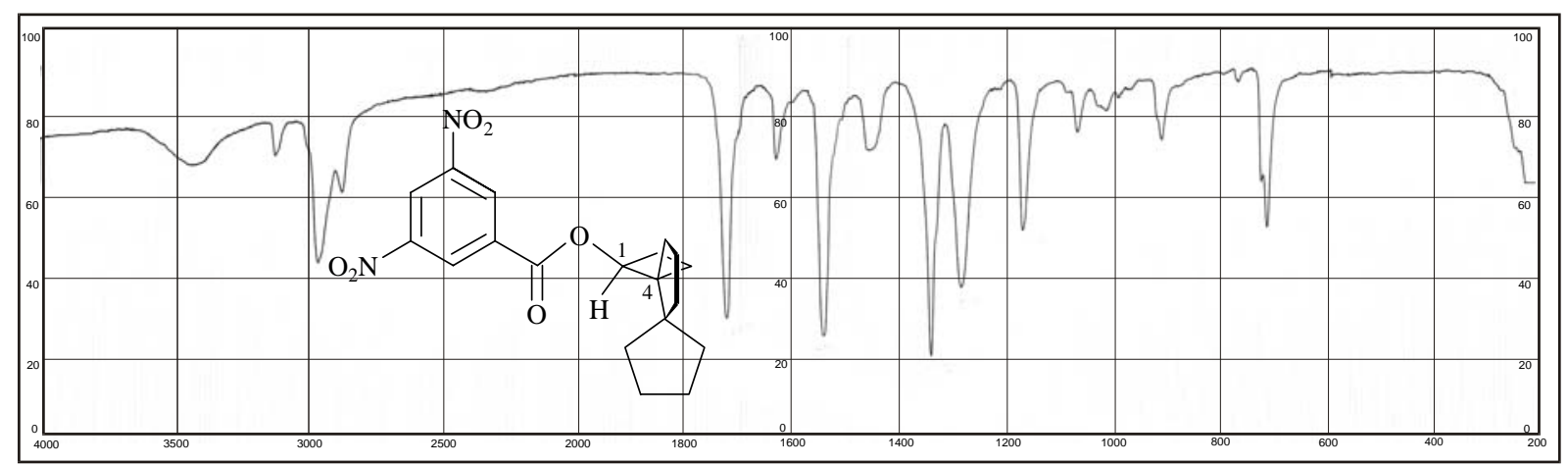

Abb. 44 IR-Spektrum von (1S,4R)-(+)-3,5-Dinitrobenzoesäure-dispiro[3.0.4.3]dodec-1yl-ester $[(1 S, 4 R)-(+)-104]$ 

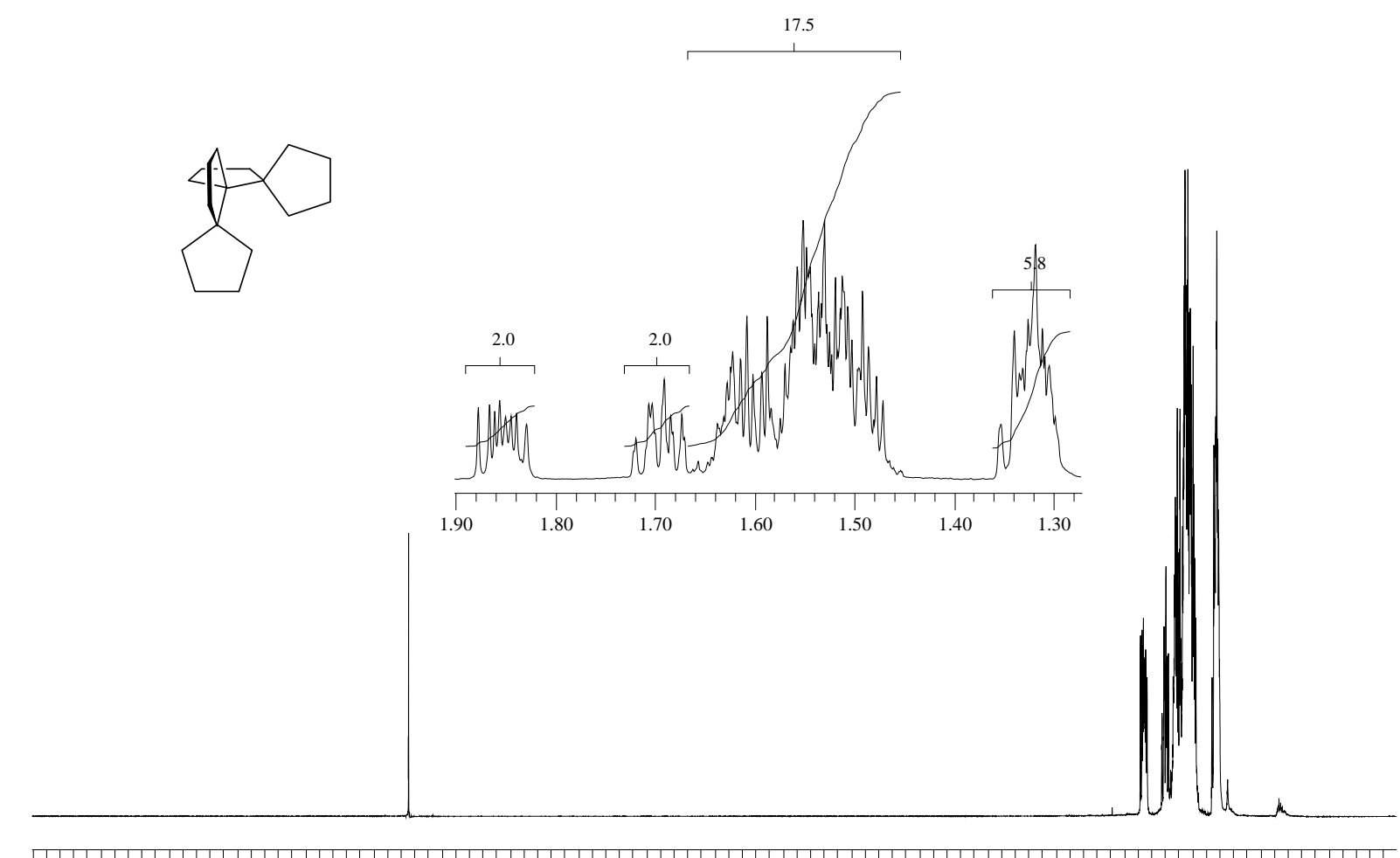

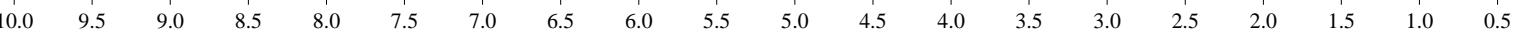

Abb. $45 \quad{ }^{1}$ H-NMR-Spektrum von Trispiro[4.0.0.4.3.3]heptadecan (8)

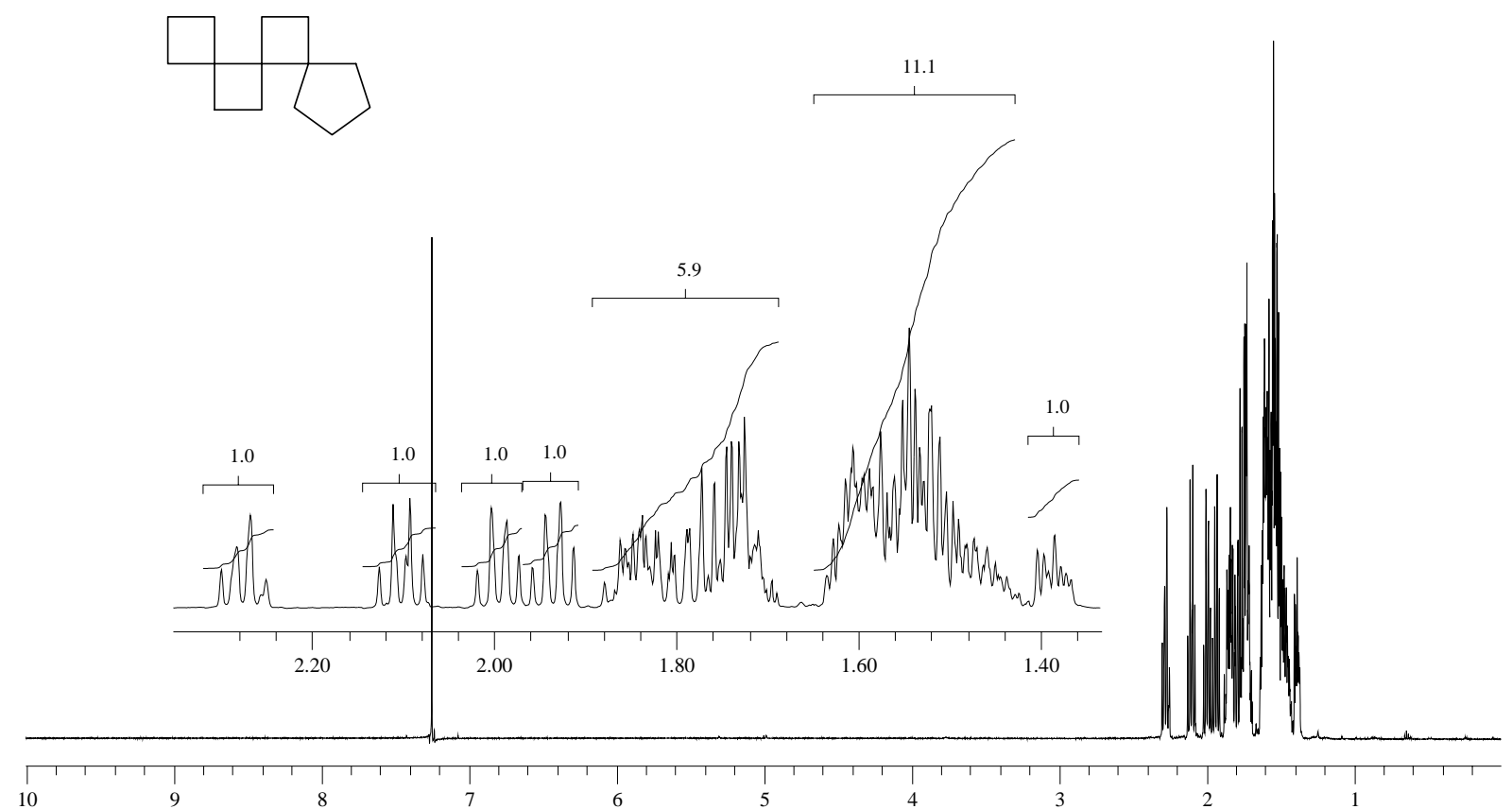

Abb. $46 \quad{ }^{1} \mathrm{H}-\mathrm{NMR}-\mathrm{Spektrum}$ von (5R*)-Trispiro[3.0.0.4.2.2]tetradecan (13) 

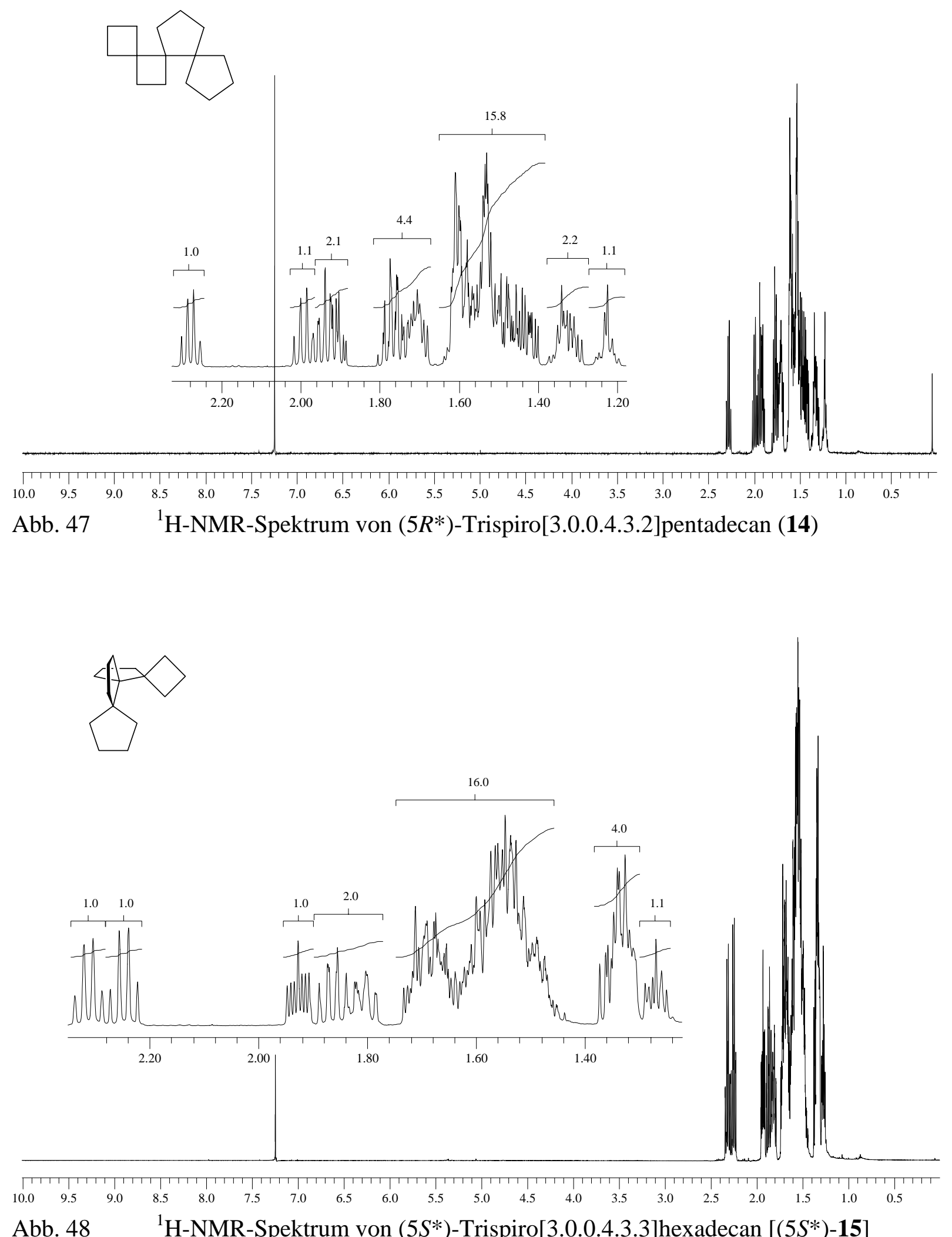

Abb. $48 \quad{ }^{1}$ H-NMR-Spektrum von (5S*)-Trispiro[3.0.0.4.3.3]hexadecan [(5S*)-15] 


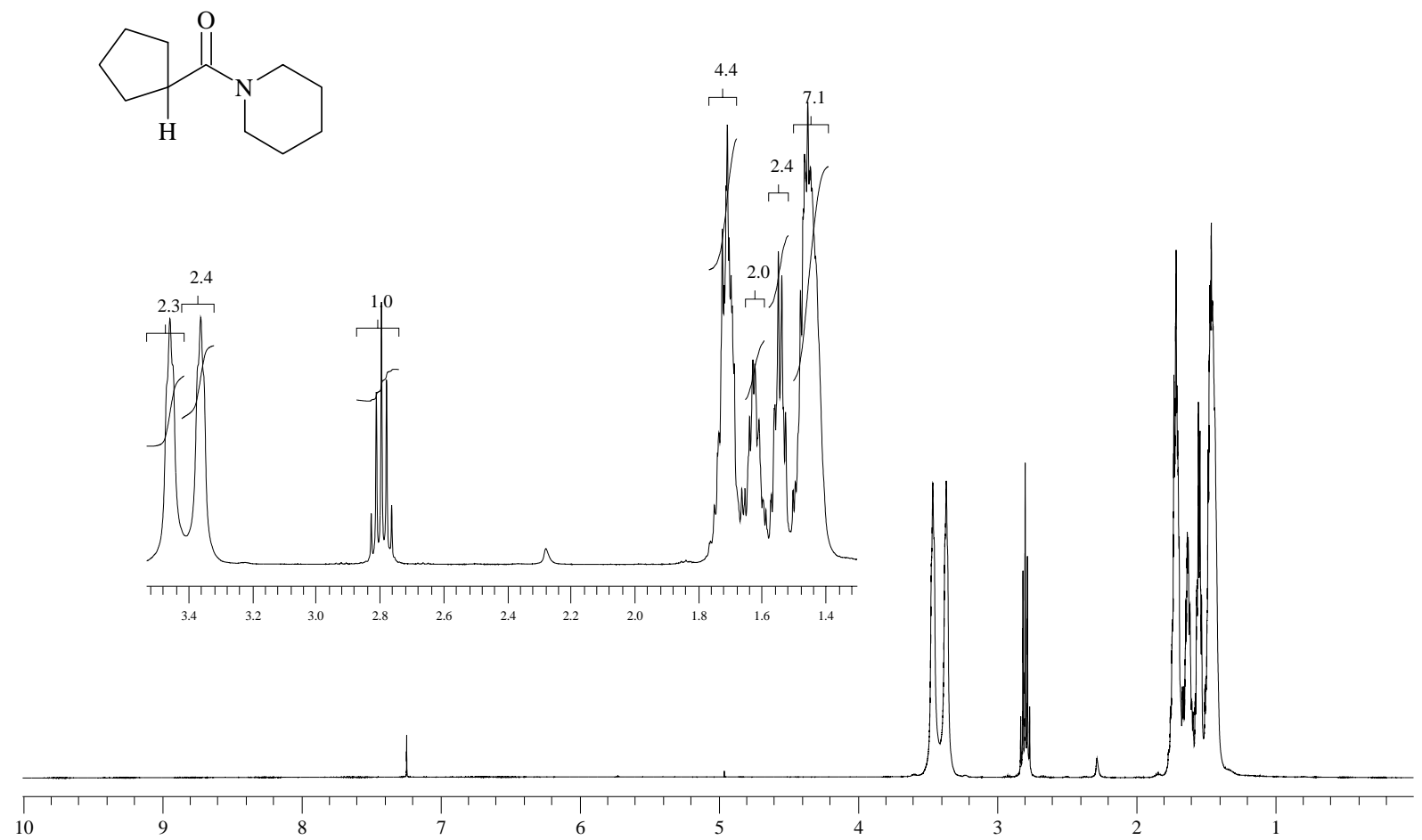

Abb. $49 \quad{ }^{1}$ H-NMR-Spektrum von 1-(Cyclopentylcarbonyl)-piperidin (23)

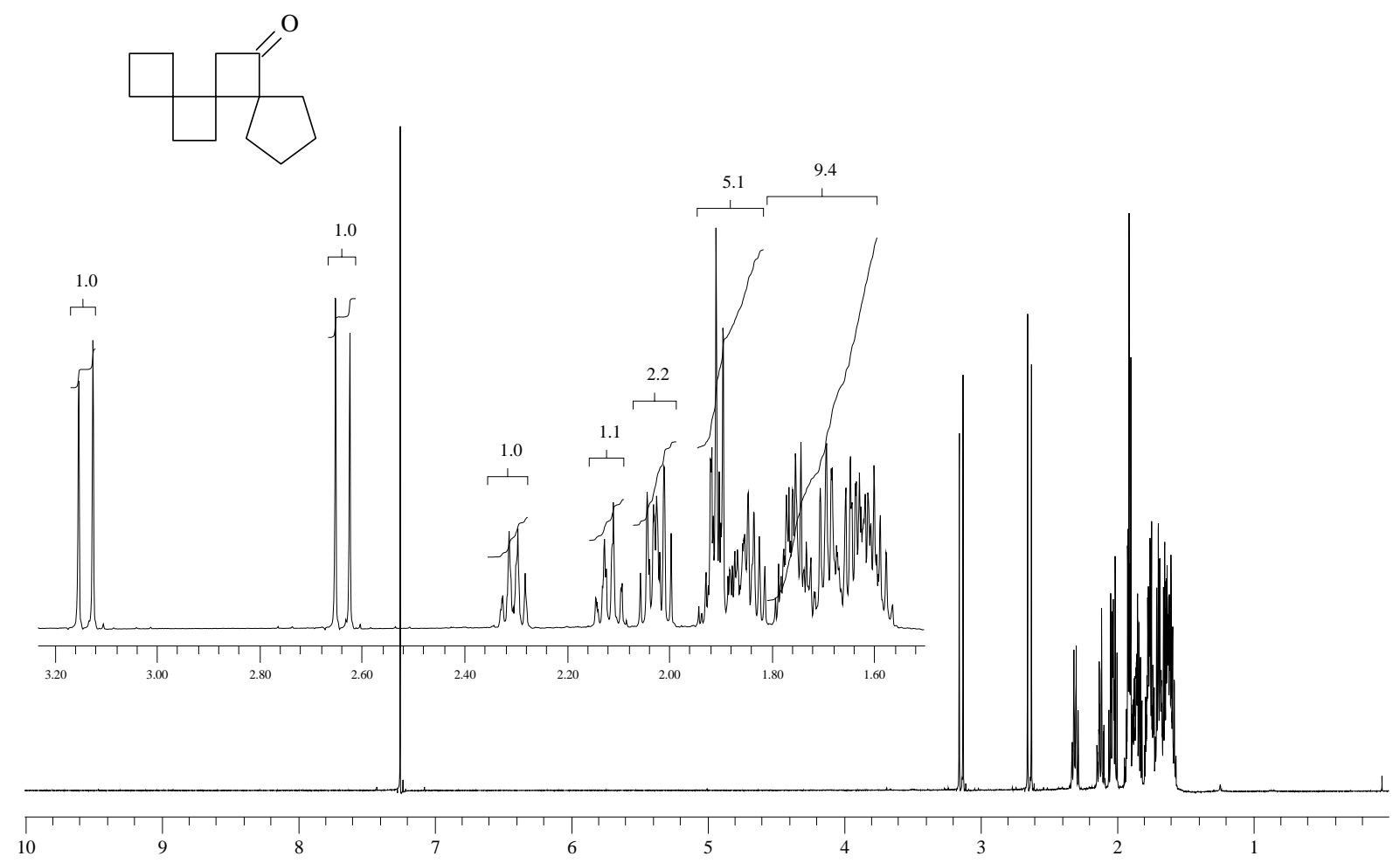

Abb. $50 \quad{ }^{1} \mathrm{H}-\mathrm{NMR}-S p e k t r u m$ von (5R*)-Trispiro[3.0.0.4.2.2]tetradecan-11-on (24) 

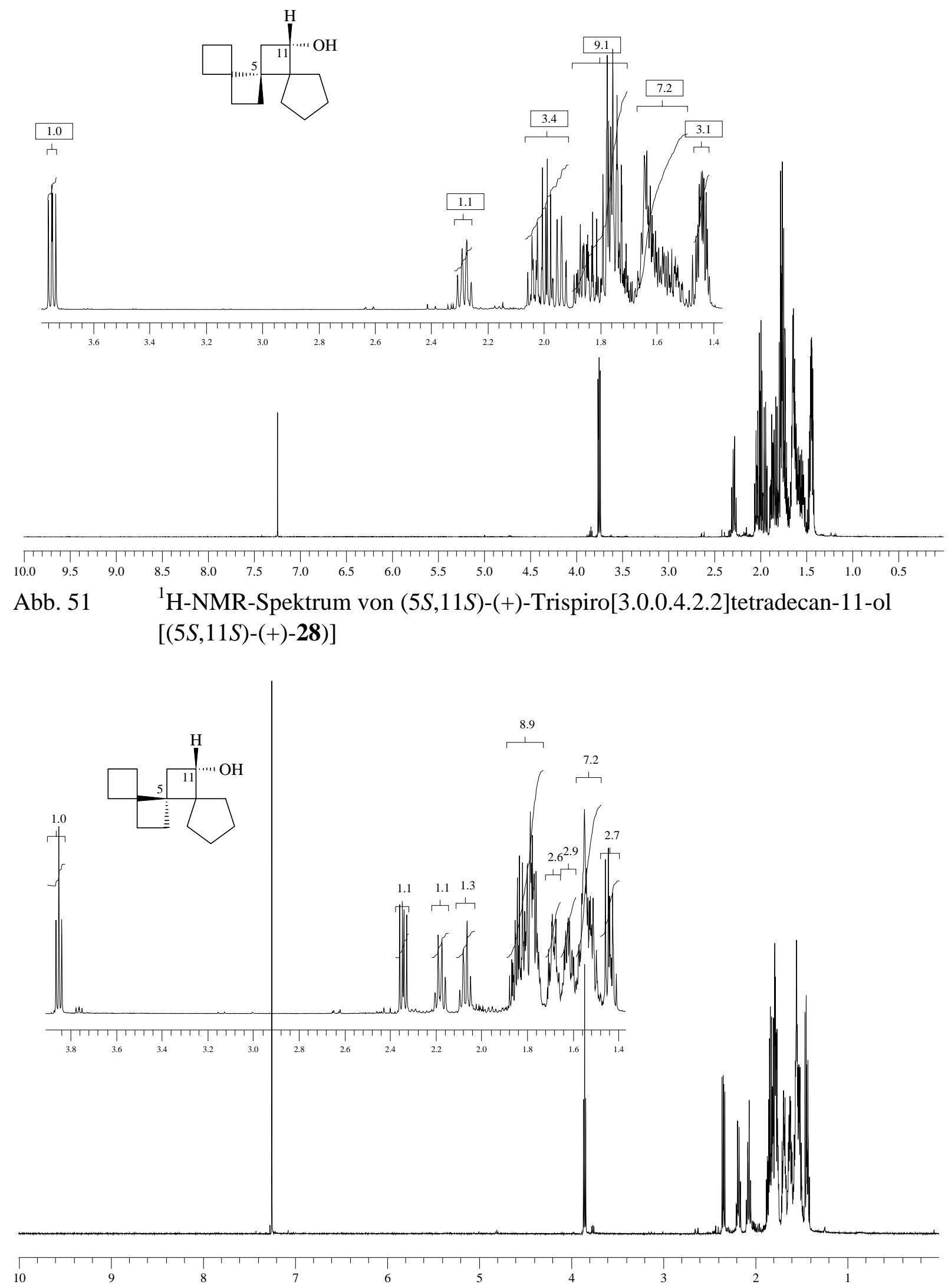

Abb. $52 \quad{ }^{1}$ H-NMR-Spektrum von (5R,11S)-(+)-Trispiro[3.0.0.4.2.2]tetradecan-11-ol [(5R,11S)-(+)-29)] 


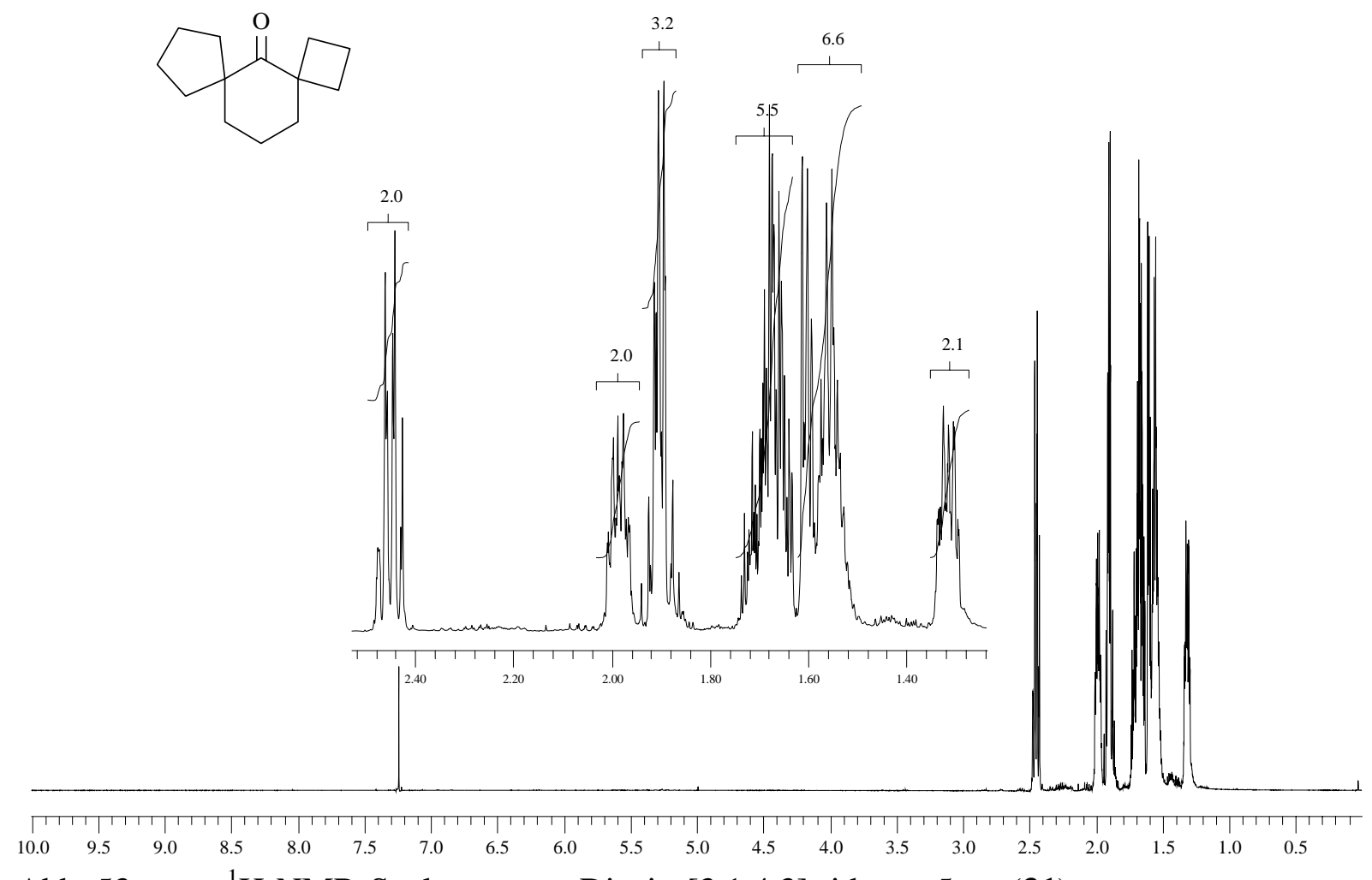

Abb. $53 \quad{ }^{1}$ H-NMR-Spektrum von Dispiro[3.1.4.3]tridecan-5-on (31)

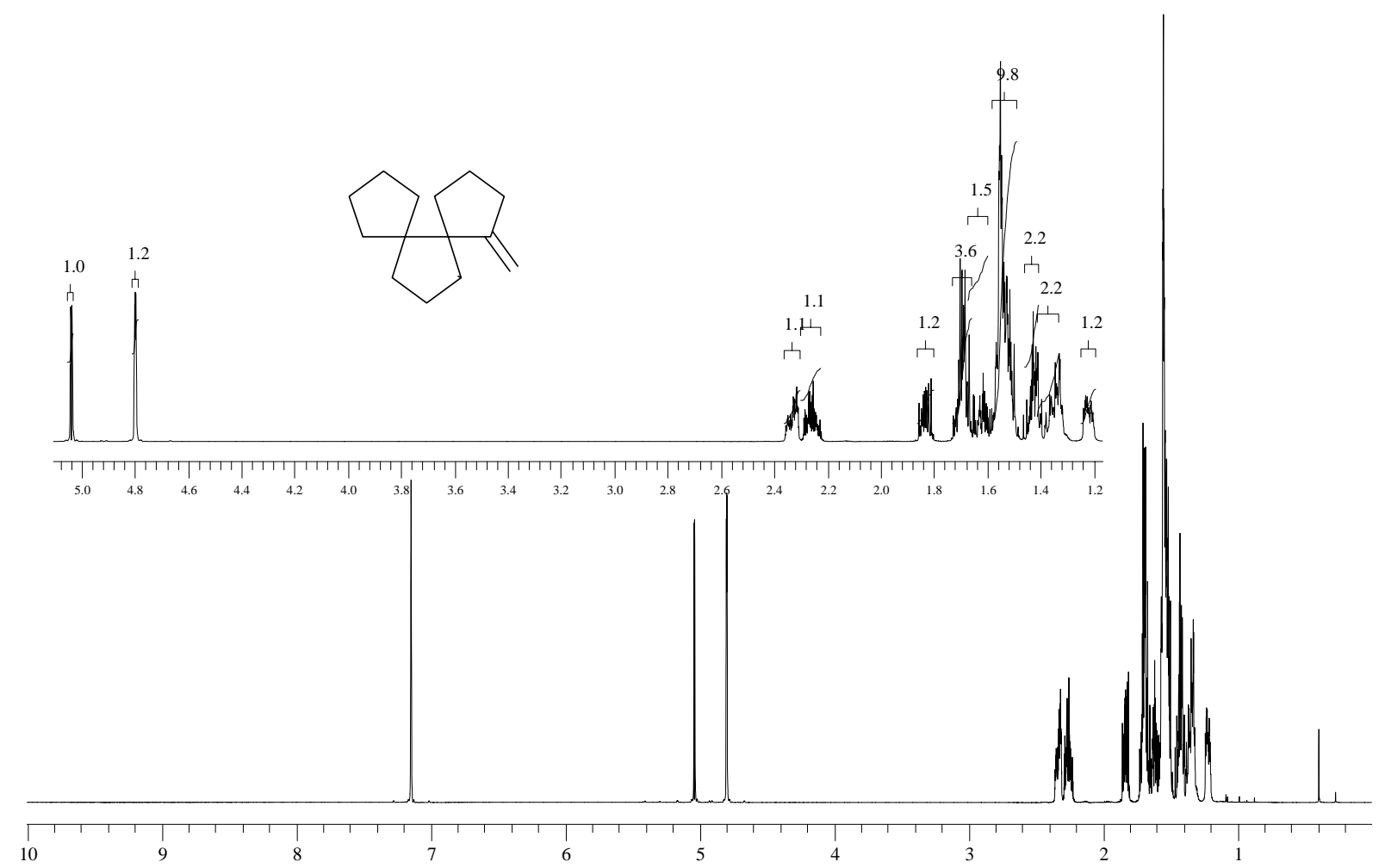

Abb. $54 \quad{ }^{1}$ H-NMR-Spektrum von 1-Methylen-dispiro[4.0.4.3]tridecan (33) 


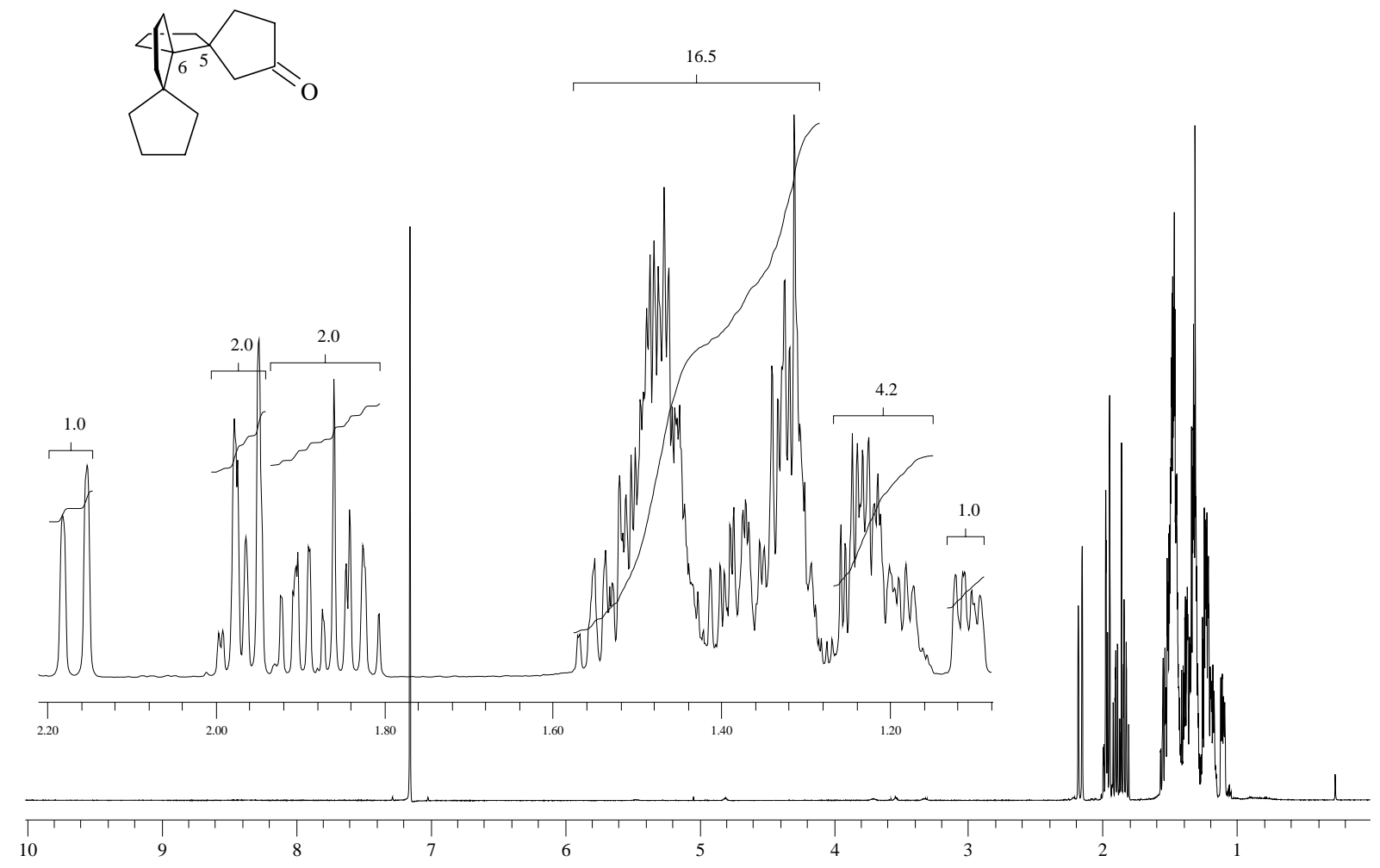

Abb. $55 \quad{ }^{1}$ H-NMR-Spektrum von (5S*,6S*)-Trispiro[4.0.0.4.3.3]heptadecan-2-on $\left[\left(5 S^{*}, 6 S^{*}\right)-35\right]$

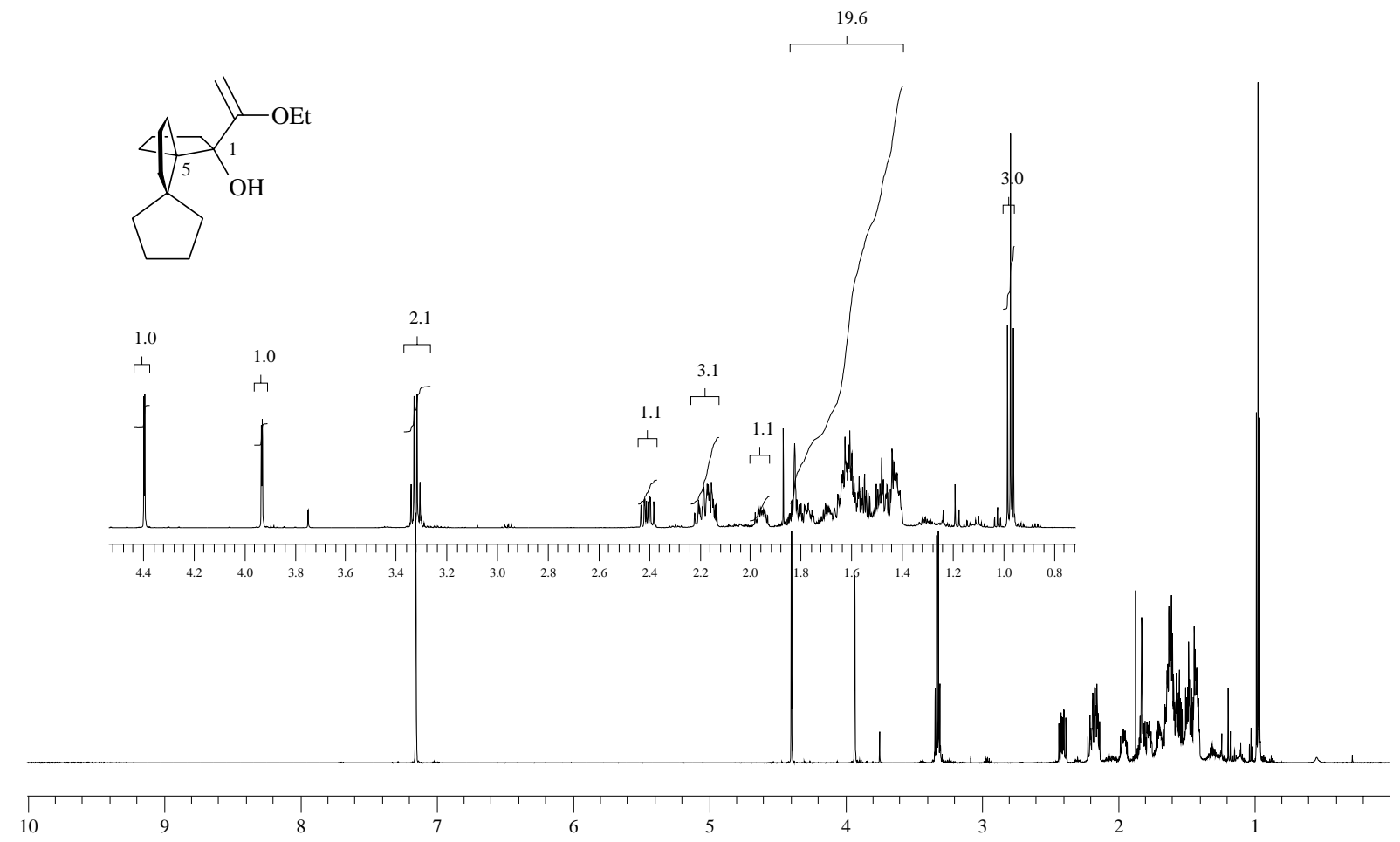

Abb. $56 \quad{ }^{1} \mathrm{H}-\mathrm{NMR}-\mathrm{Spektrum}$ von $\left(1 S^{*}, 5 S^{*}\right)$-1-(1-Ethoxy-vinyl)-dispiro[4.0.4.3]tridecan$1-o l\left[\left(1 S^{*}, 5 S^{*}\right)-41\right]$ 

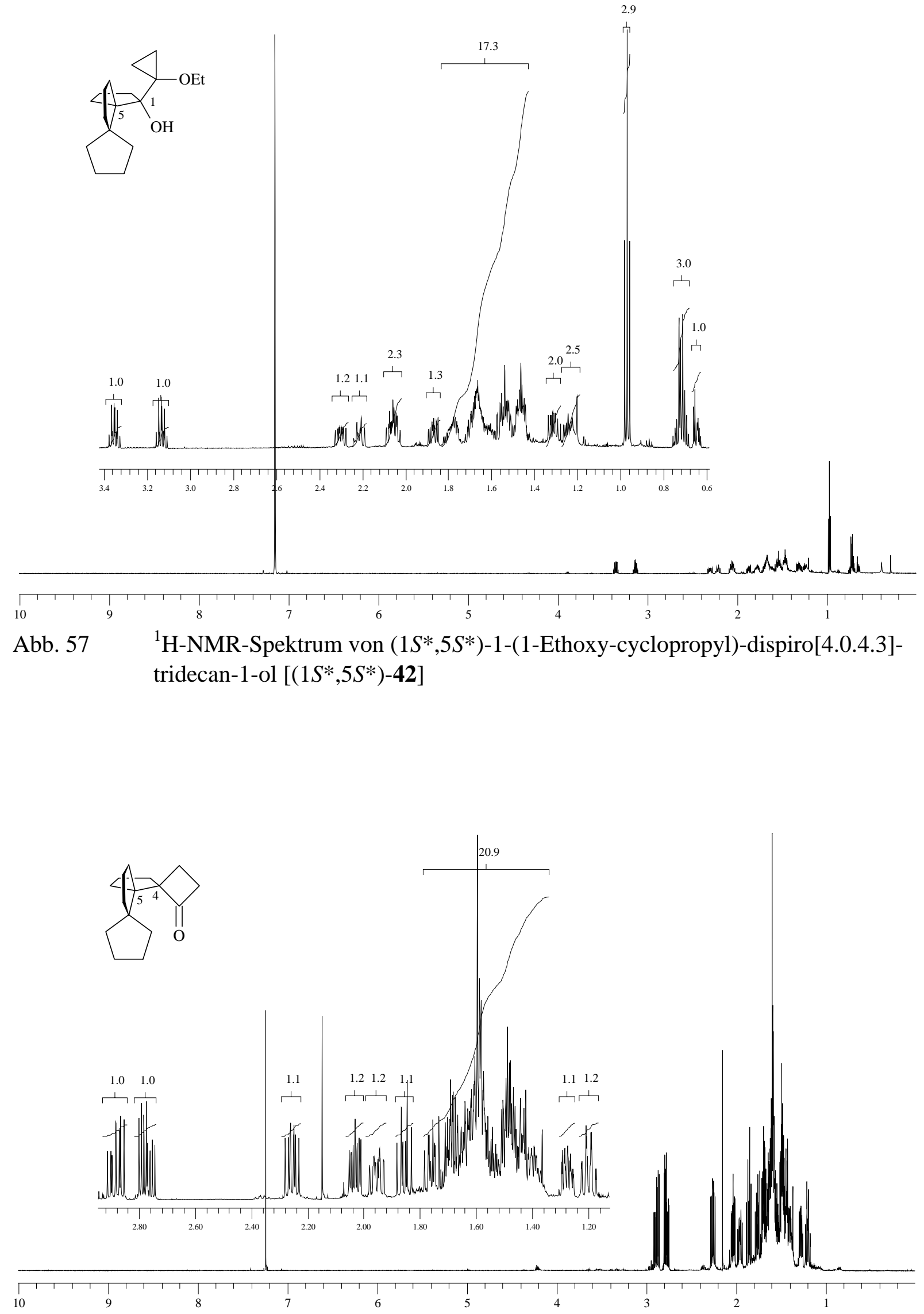

Abb. $58 \quad{ }^{1} \mathrm{H}-\mathrm{NMR}-\mathrm{Spektrum}$ von $\left(4 R^{*}, 5 S^{*}\right)$-Trispiro[3.0.0.4.3.3] [(4R*,5S*)-43] 


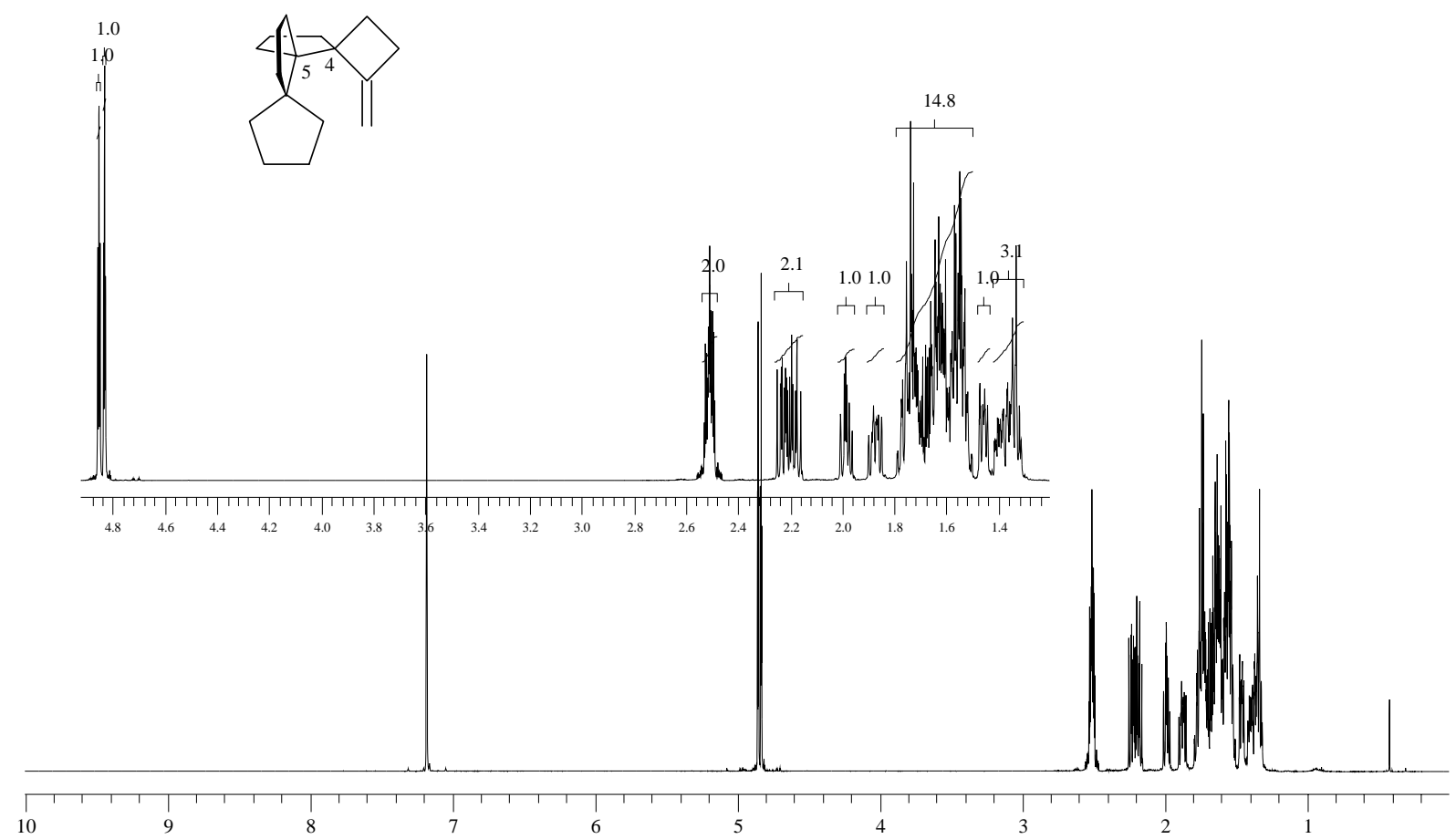

Abb. $59{ }^{1} \mathrm{H}$-NMR-Spektrum von $\left(4 R^{*}, 5 S^{*}\right)$-1-Methylen-trispiro[3.0.0.4.3.3] hexadecan $\left[\left(4 R^{*}, 5 S^{*}\right)-44\right]$

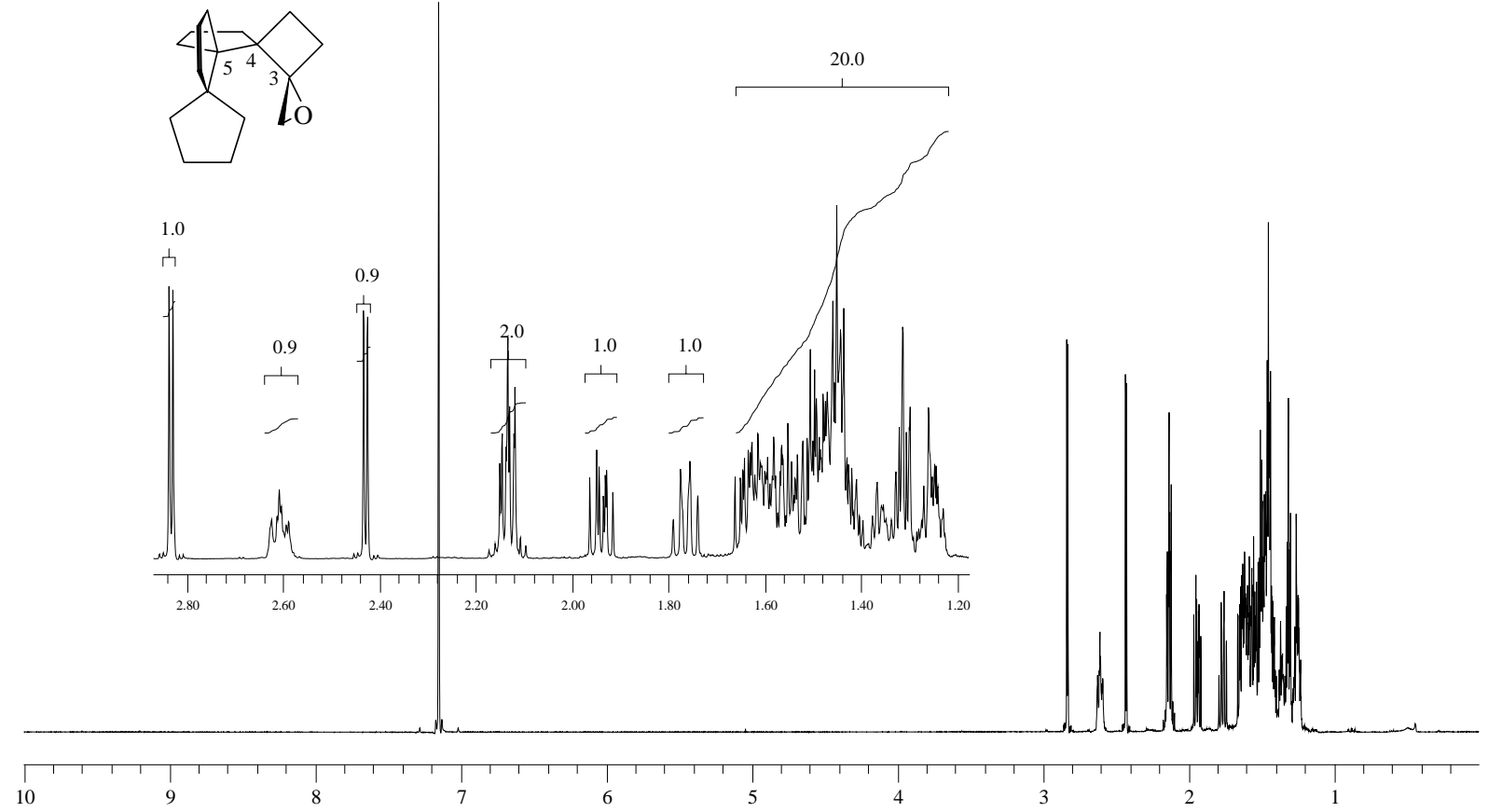

Abb. $60 \quad{ }^{1} \mathrm{H}-\mathrm{NMR}-S p e k t r u m$ von $\left(3 S^{*}, 4 R^{*}, 5 S^{*}\right)-1-O x a-t e t r a s p i r o[2.0 .0 .0 .4 .3 .3 .2]$ octadecan $\left[\left(3 S^{*}, 4 R^{*}, 5 S^{*}\right)-46\right]$ 

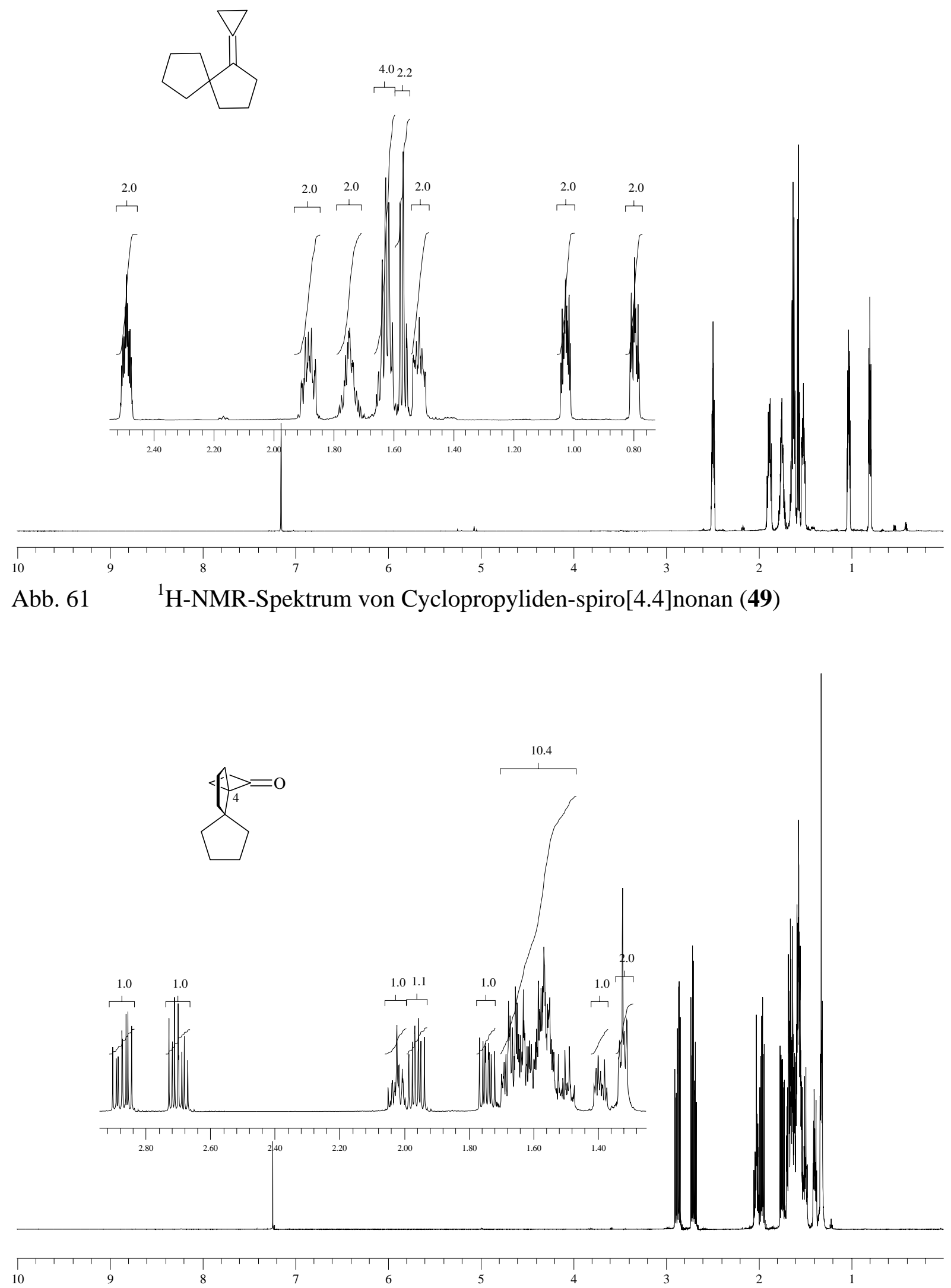

Abb. $62 \quad{ }^{1}$ H-NMR-Spektrum von (4S*)-Dispiro[3.0.4.3]dodecan-1-on [(4S*)-51] 


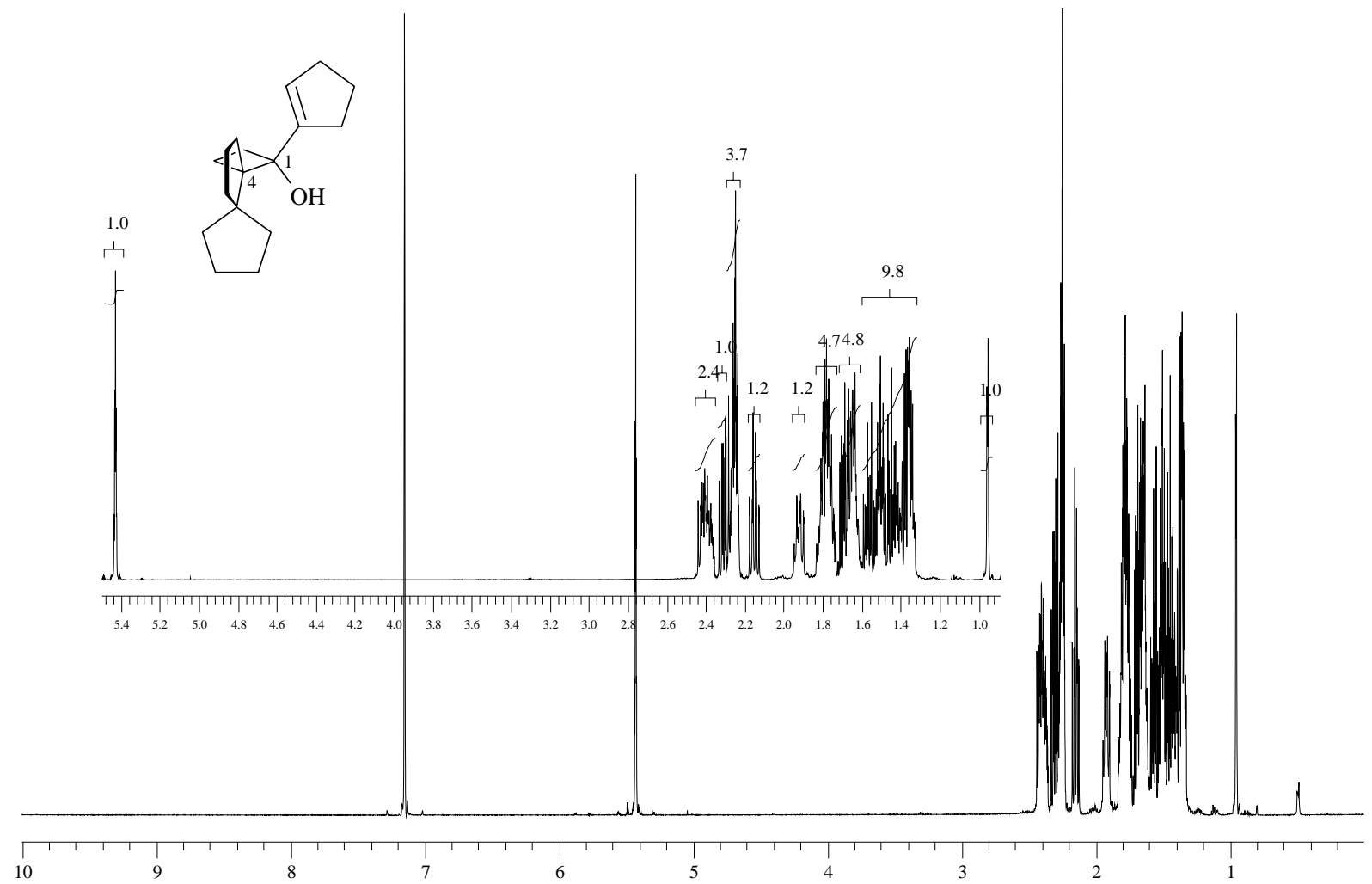

Abb. $63 \quad{ }^{1}$ H-NMR-Spektrum von $\left(1 R^{*}, 4 S^{*}\right)-1-C y c l o p e n t-1-e n y l-d i s p i r o[3.0 .4 .3] d o d e-$ can-1-ol [(1R*,4S*)-52]

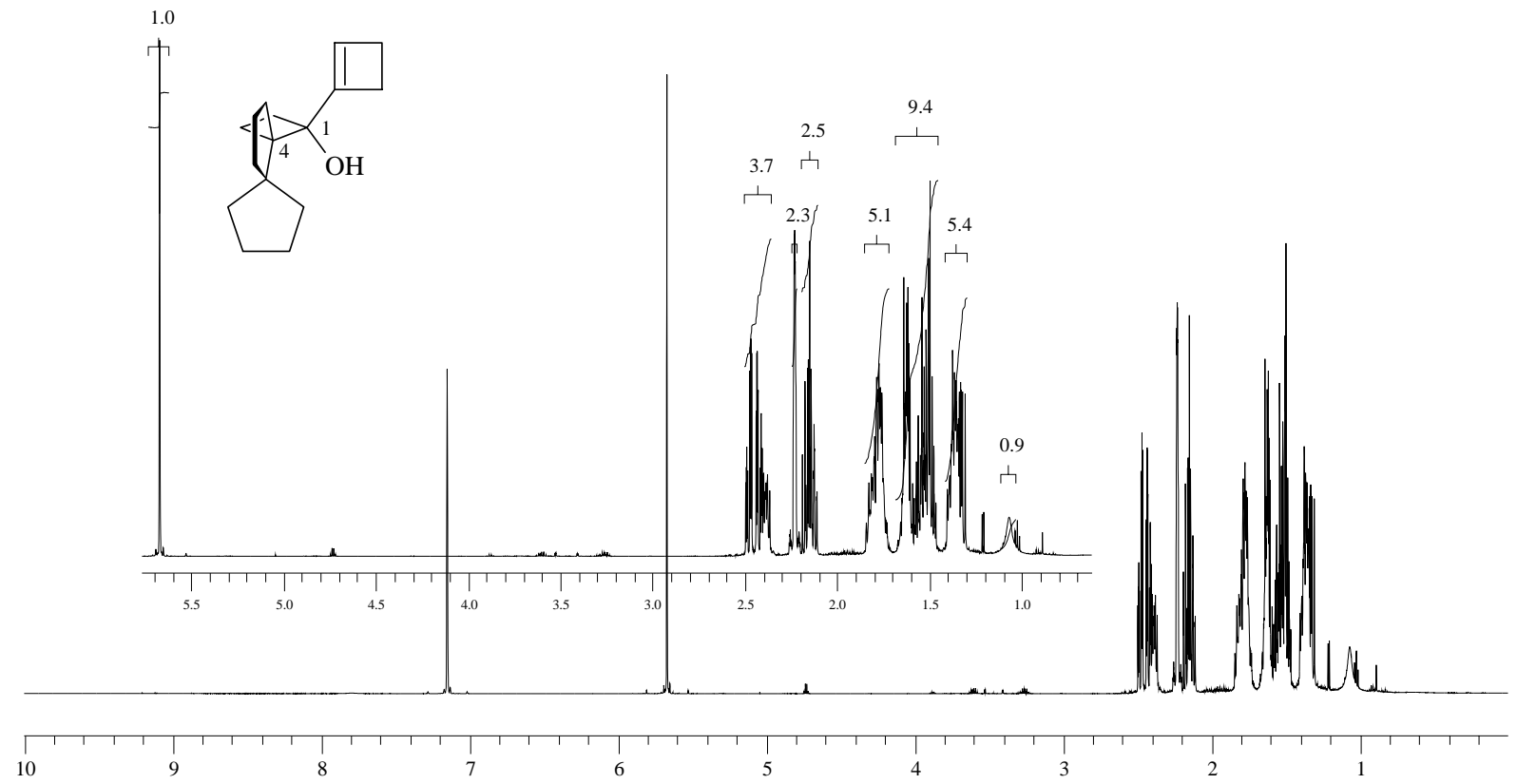

Abb. $64 \quad{ }^{1}$ H-NMR-Spektrum von $\left(1 R^{*}, 4 S^{*}\right)$-1-Cyclobut-1-enyl-dispiro[3.0.4.3]dodecan$1-\mathrm{ol}\left[\left(1 R^{*}, 4 S^{*}\right)-53\right]$ 


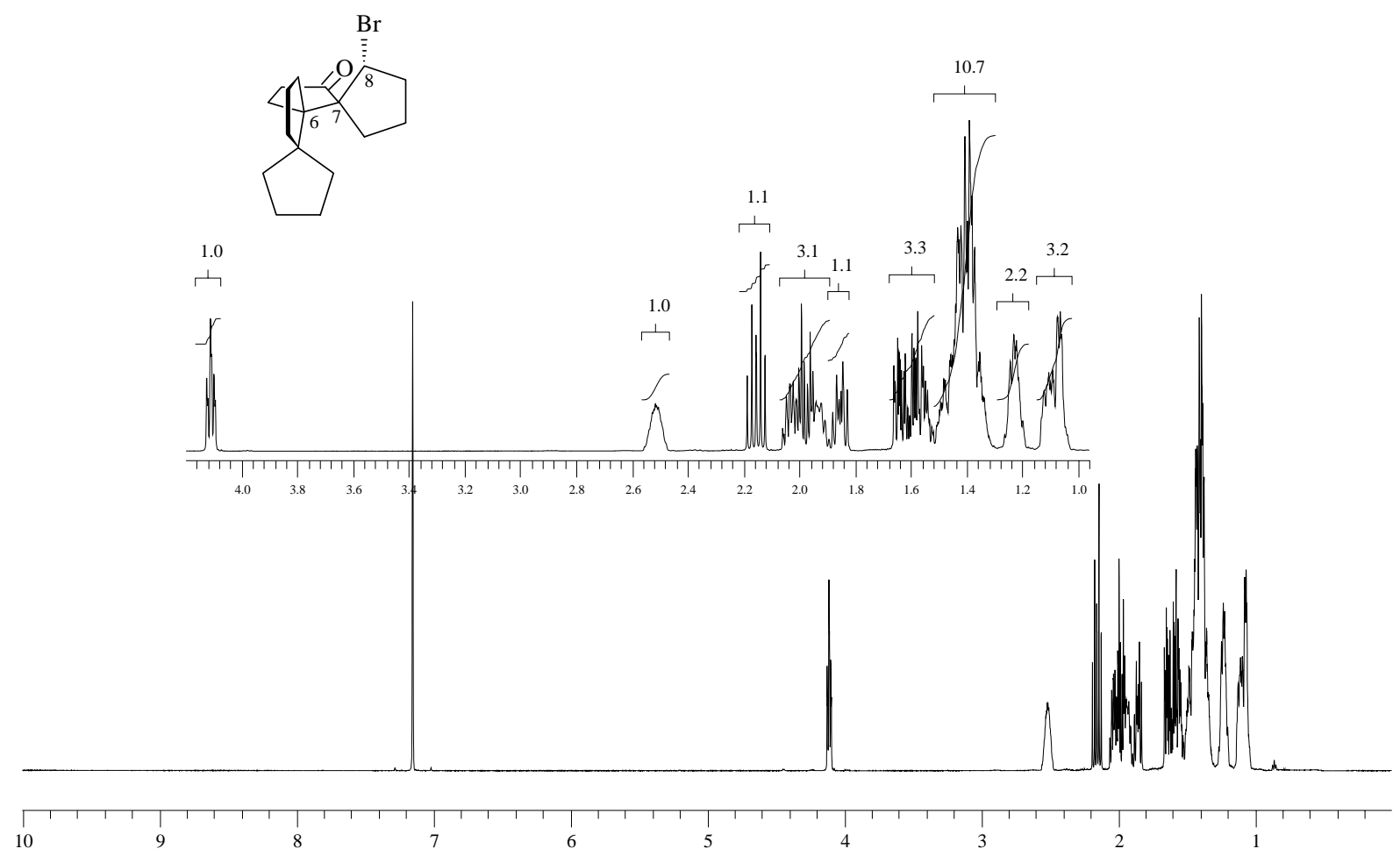

Abb. $65 \quad{ }^{1} \mathrm{H}-\mathrm{NMR}-S p e k t r u m$ von $\left(6 S^{*}, 7 S^{*}, 8 R^{*}\right)$-8-Brom-trispiro[4.0.0.4.3.3]heptadecan-12-on [(6S*,7S*,8R*)-54]

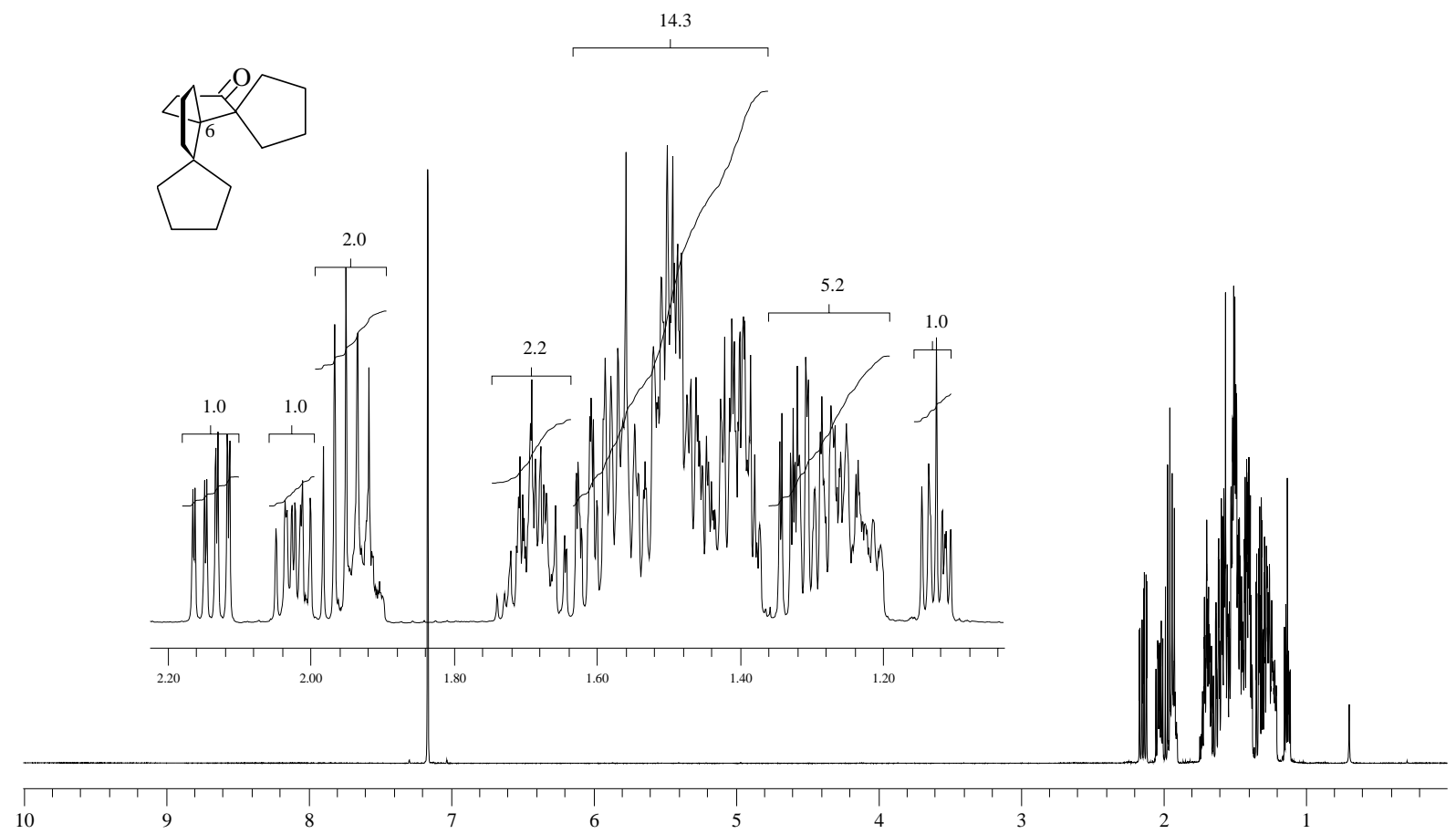

Abb. $66 \quad{ }^{1}$ H-NMR-Spektrum von (6S*)-Trispiro[4.0.0.4.3.3] heptadecan-12-on [(6 $\left.6 S^{*}\right)$ 55] 


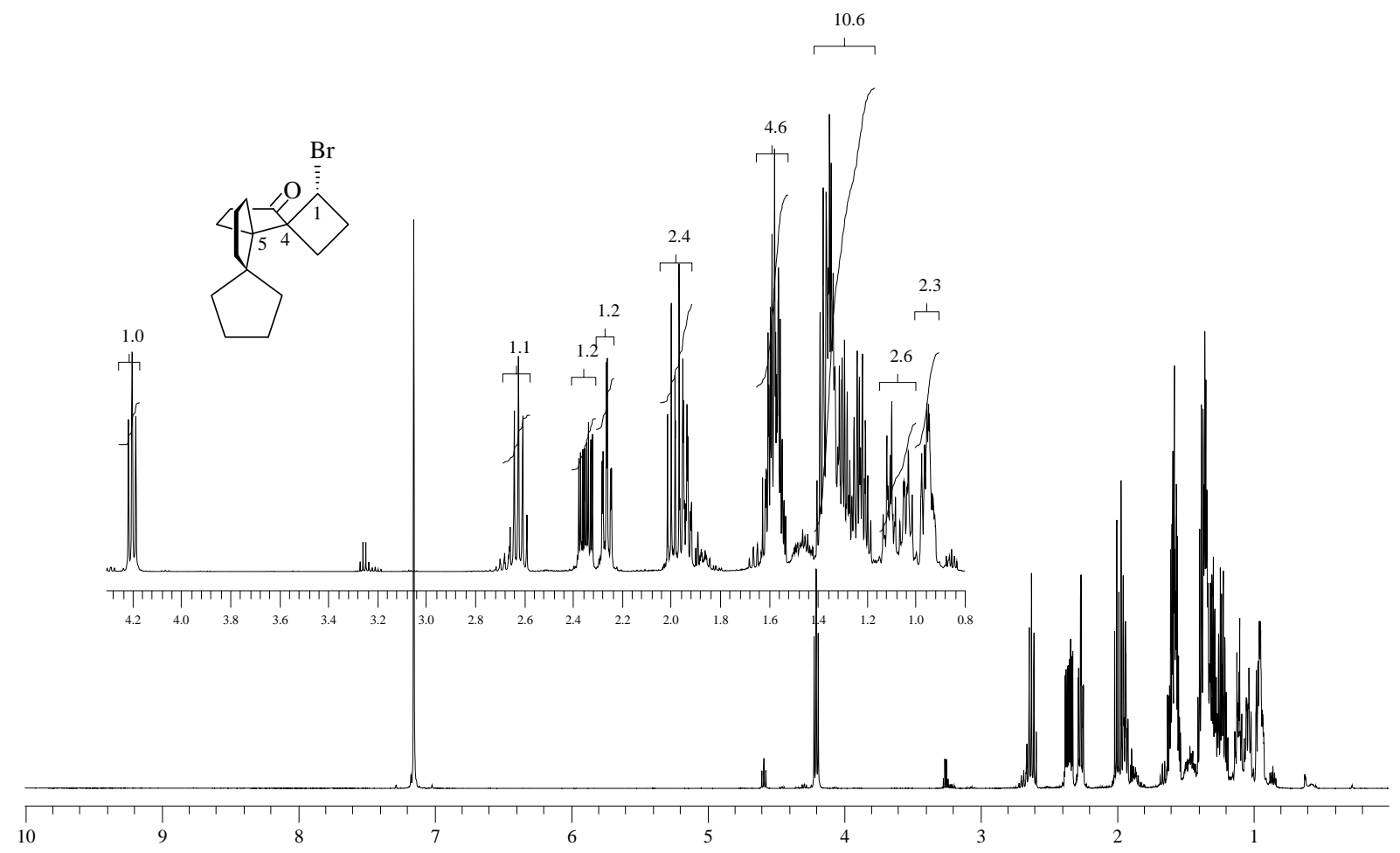

Abb. $67 \quad{ }^{1} \mathrm{H}-\mathrm{NMR}-S p e k t r u m$ von $\left(1 R^{*}, 4 S^{*}, 5 S^{*}\right)$-1-Brom-trispiro[3.0.0.4.3.3] hexadecan16-on $\left[\left(1 R^{*}, 4 S^{*}, 5 S^{*}\right)-56\right]$
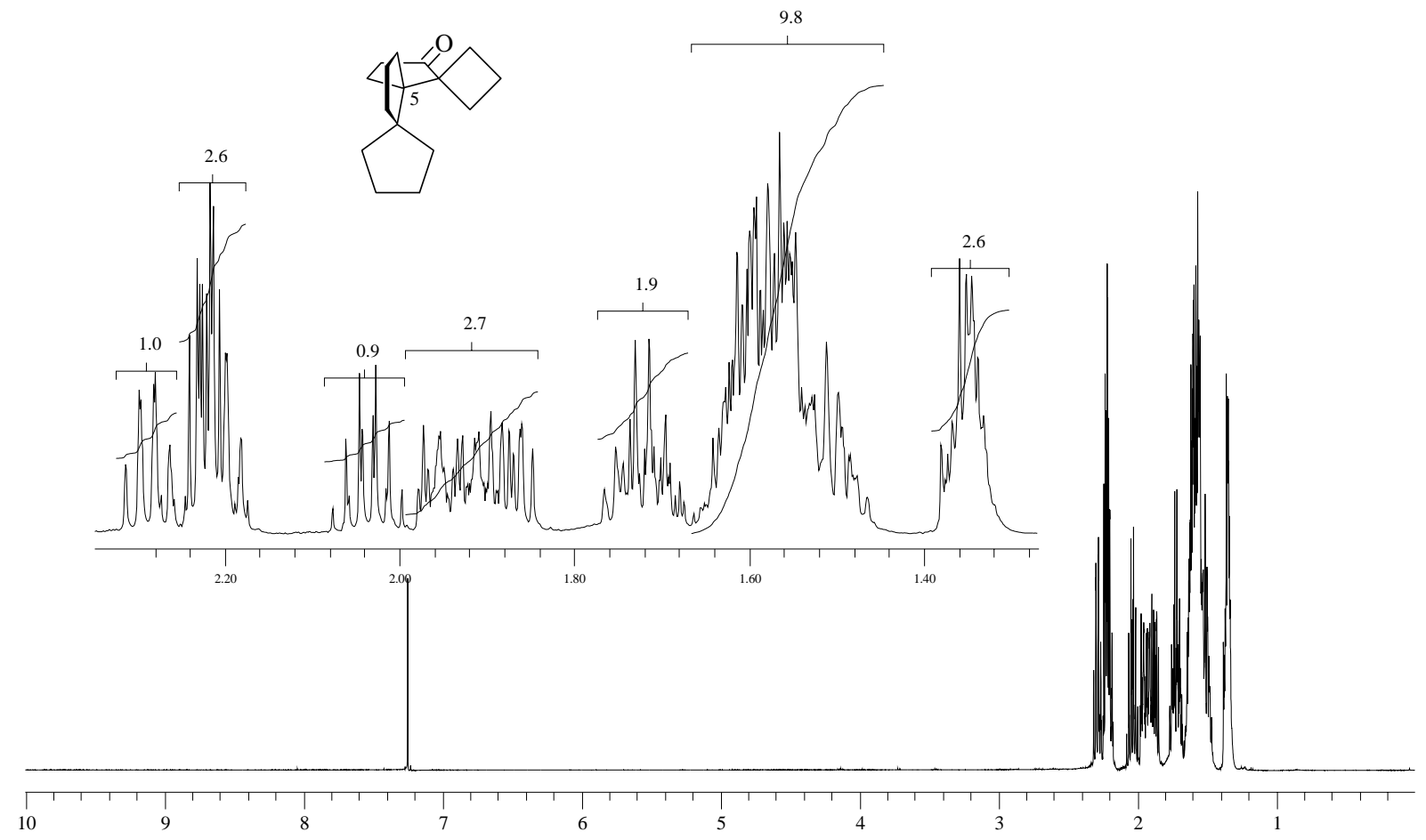

Abb. $68 \quad{ }^{1} \mathrm{H}-\mathrm{NMR}-\mathrm{Spektrum}$ von (5S*)-Trispiro[3.0.0.4.3.3] hexadecan-16-on [(5S*)-57] 


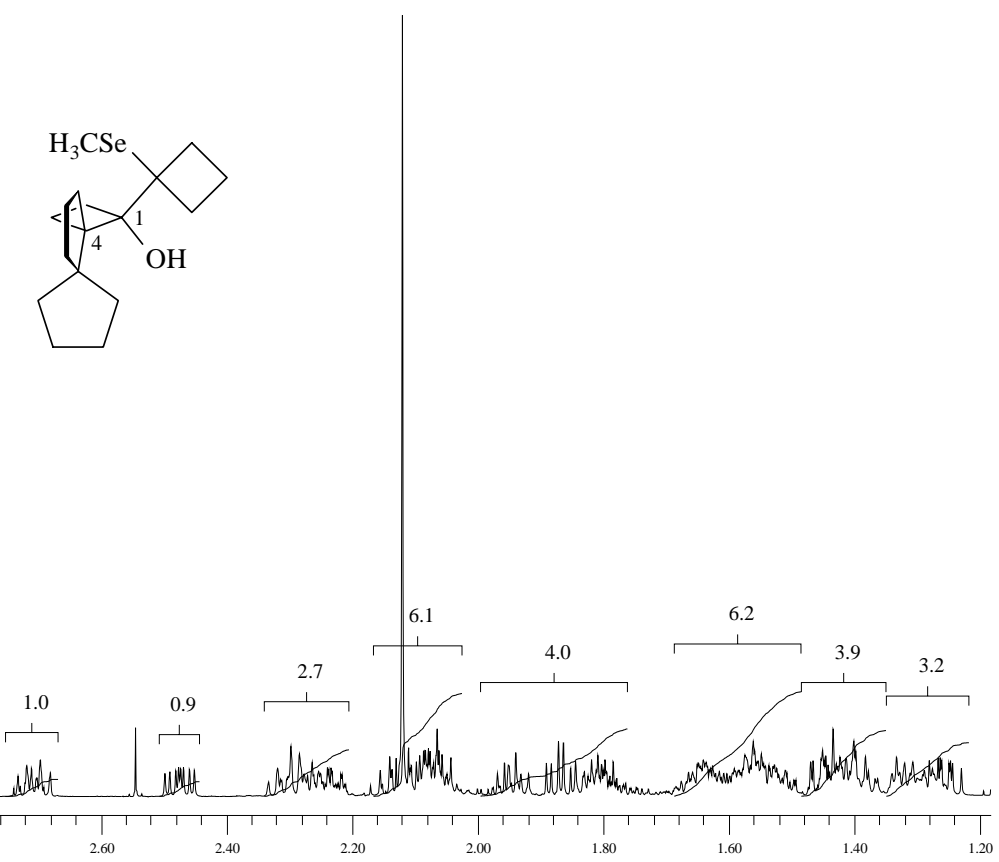

hathinghin

Abb. $69 \quad{ }^{1} \mathrm{H}-\mathrm{NMR}-\mathrm{Spektrum}$ von $\left(1 S^{*}, 4 S^{*}\right)-1-\left[\left(1^{\prime}\right.\right.$-Methylselanyl)cyclobutyl)]dispiro[3.0.4.3]dodecan-1-ol [(1S*,4S*)-58]

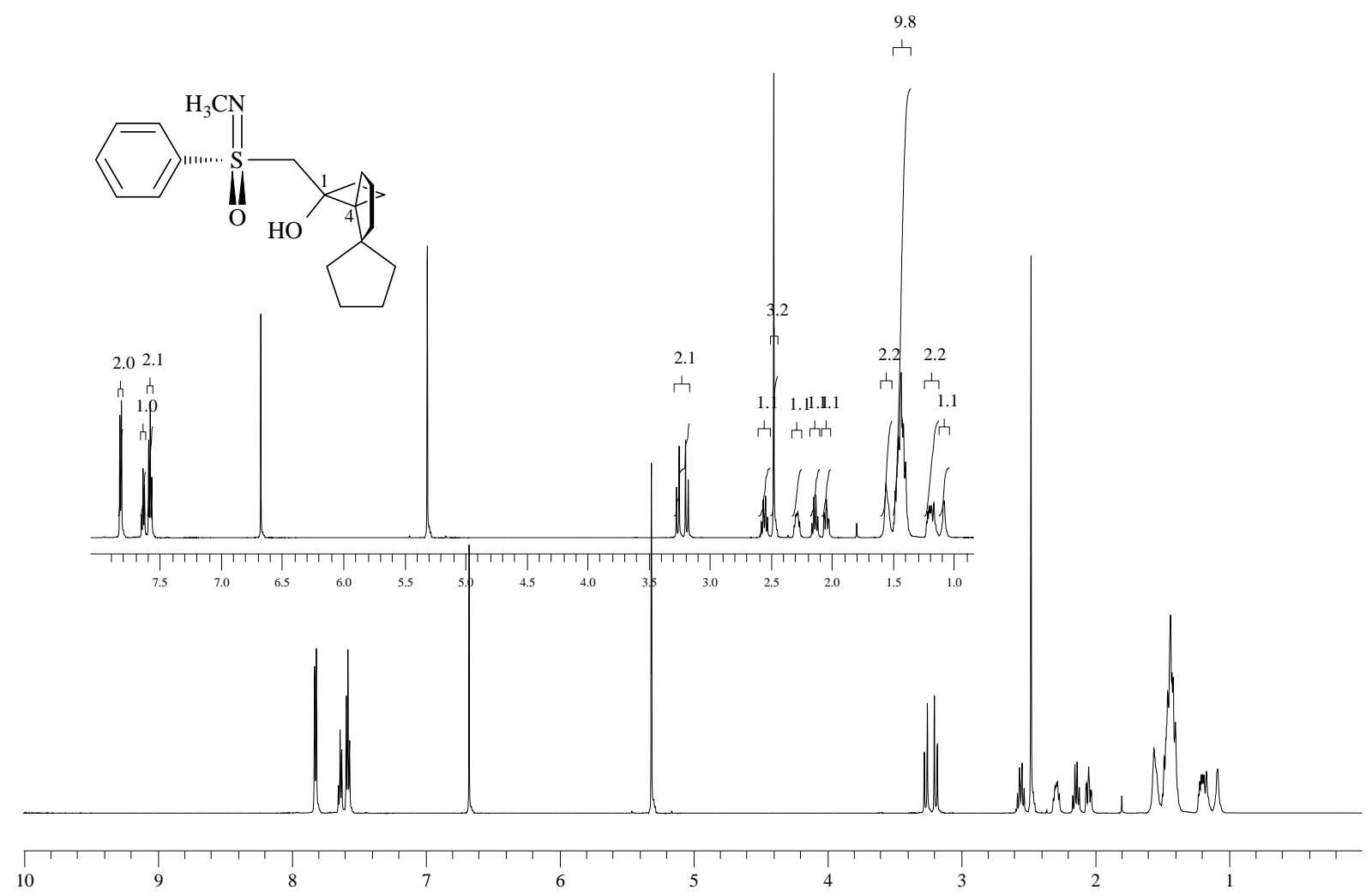

Abb. $70 \quad{ }^{1} \mathrm{H}-\mathrm{NMR}-S p e k t r u m$ von (SS,1R,4R)-(+)-[(N-Methyl-S-phenylsulfonimidoyl)methyl]-dispiro[3.0.4.3]dodecan-1-ol [(SS,1R,4R)-(+)-59] 


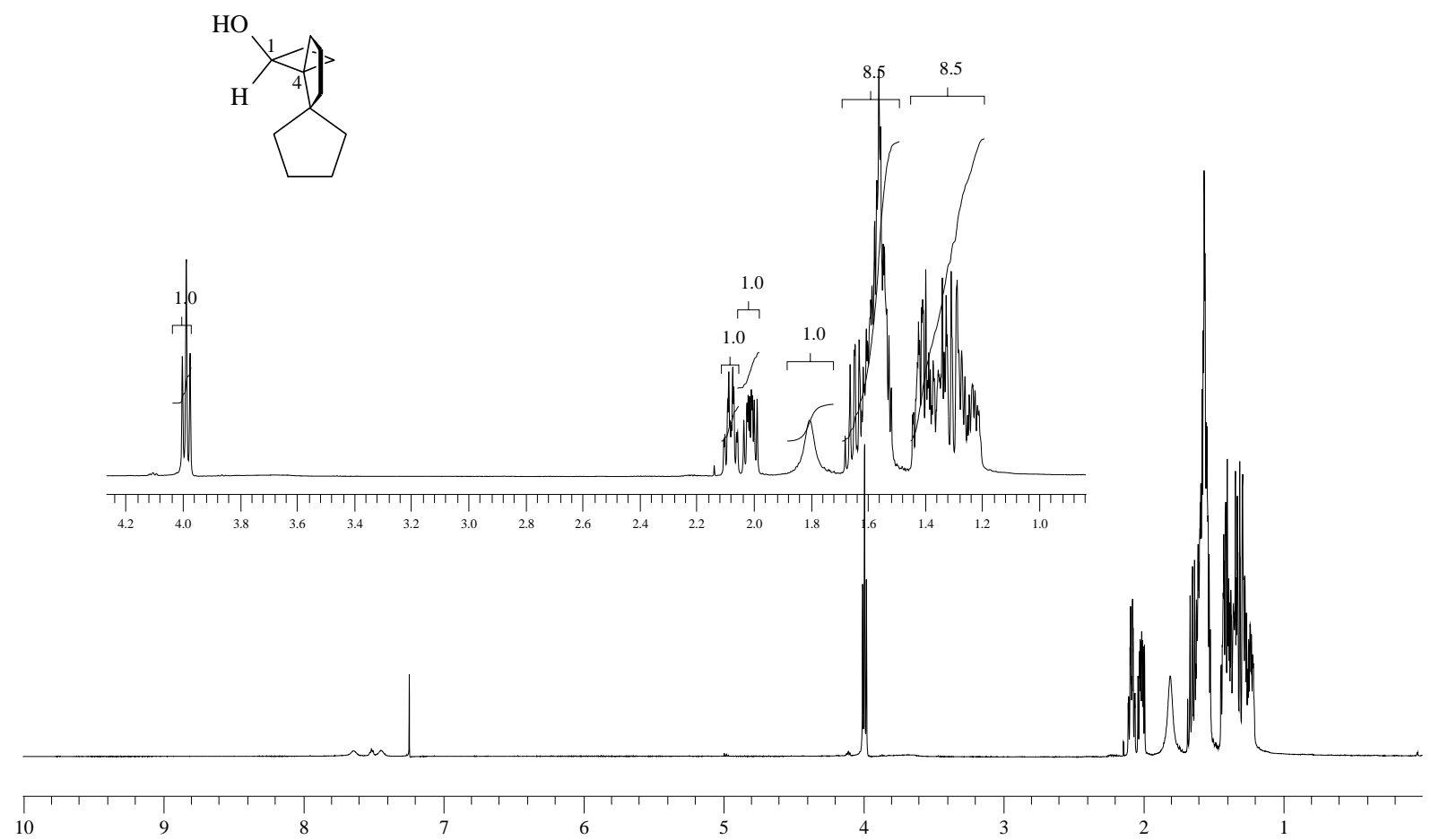

Abb. $71 \quad{ }^{1}$ H-NMR-Spektrum von (1S,4R)-(+)-Dispiro[3.0.4.3]dodecan-1-ol [(1S,4R)$(+)-60]$

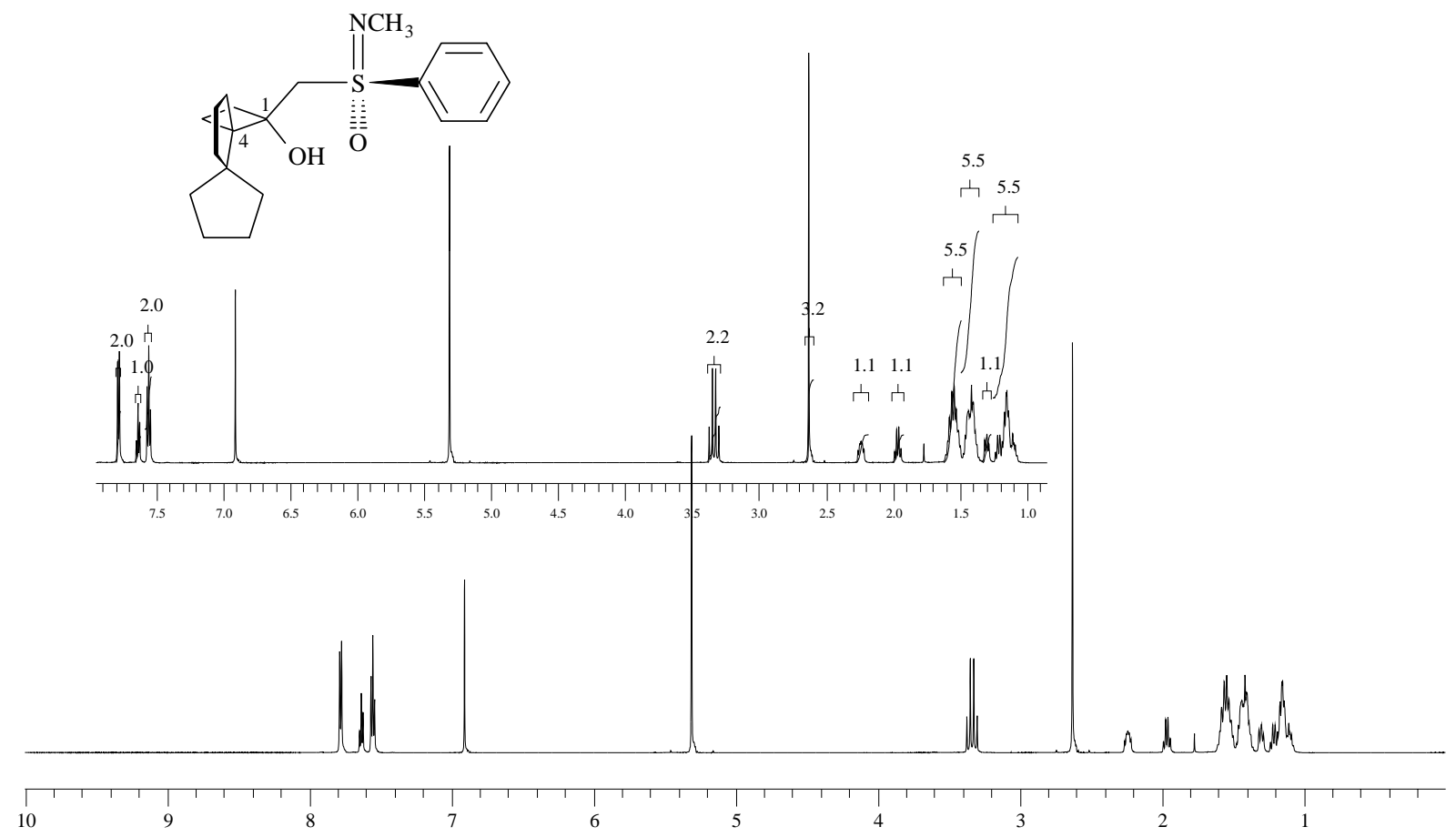

Abb. $72 \quad{ }^{1}$ H-NMR-Spektrum von (SS,1S,4S)-(+)-[(N-Methyl-S-phenylsulfonimidoyl)methyl]-dispiro[3.0.4.3]dodecan-1-ol [(SS,1S,4S)-(+)-61] 


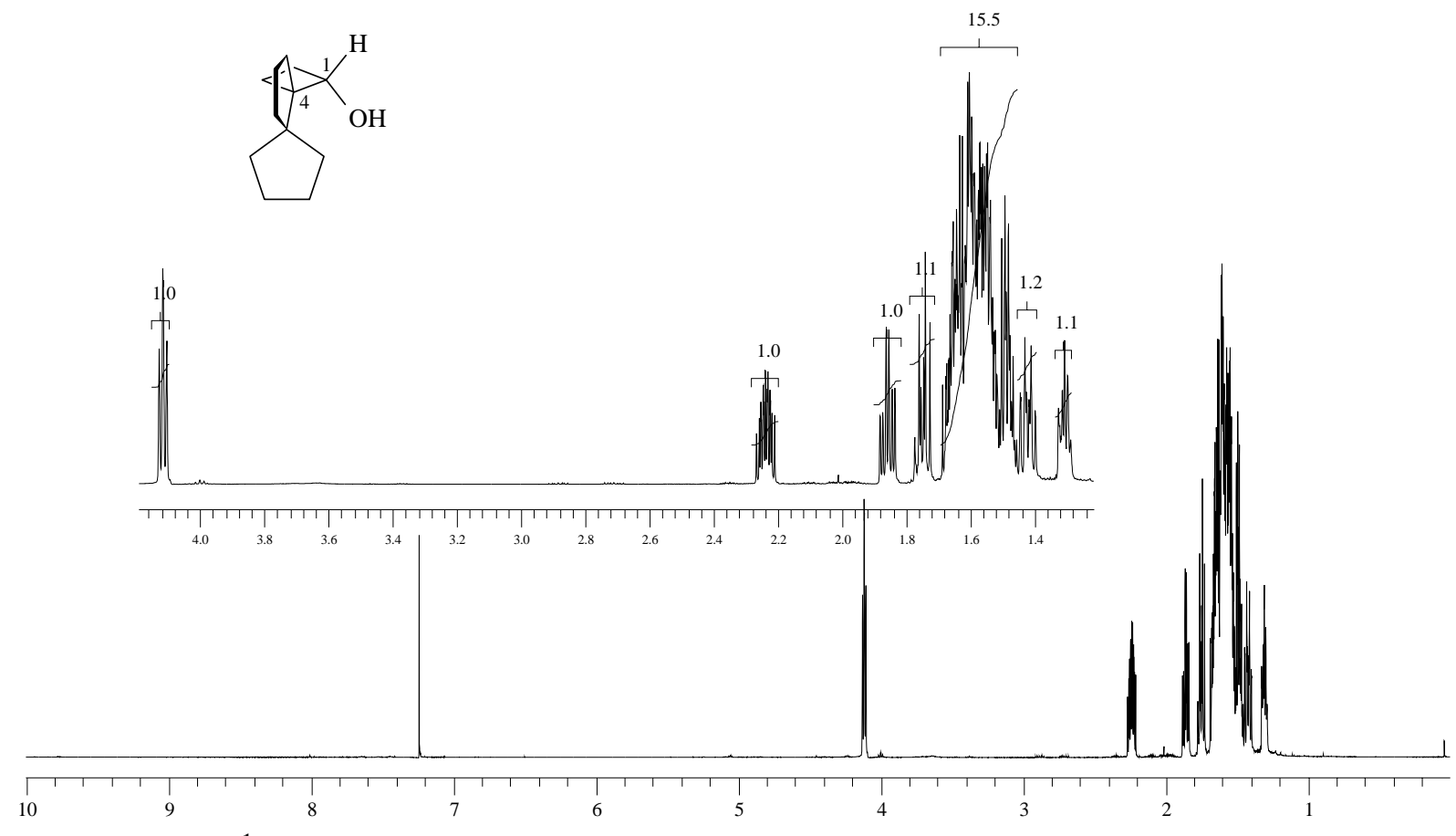

Abb. $73 \quad{ }^{1}$ H-NMR-Spektrum von $(1 S, 4 S)-(+)-D i s p i r o[3.0 .4 .3]$ dodecan-1-ol [(1S,4S)-(+)62]
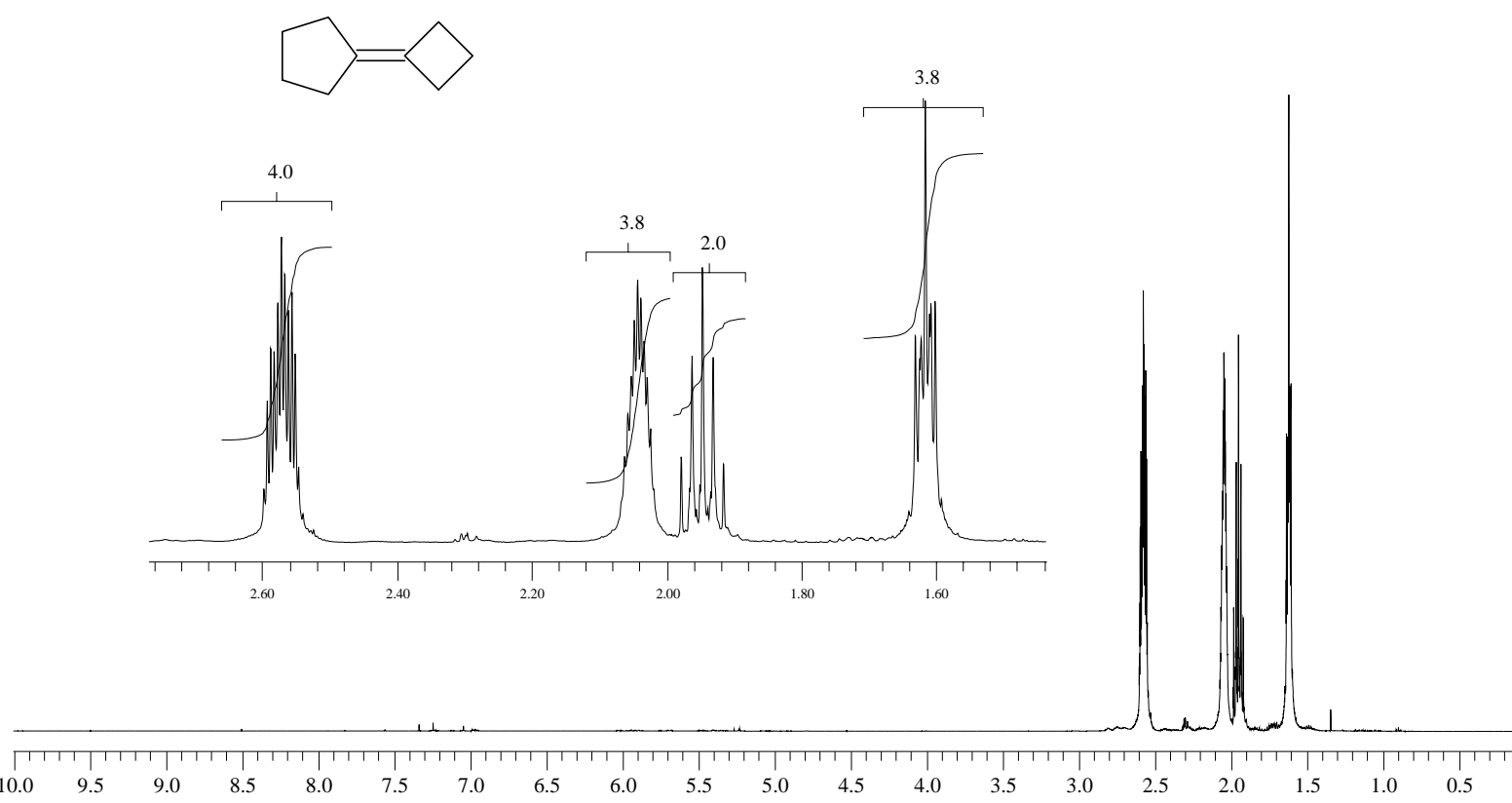

Abb. $74 \quad{ }^{1}$ H-NMR-Spektrum von Cyclobutyliden-Cyclopentan (69) 

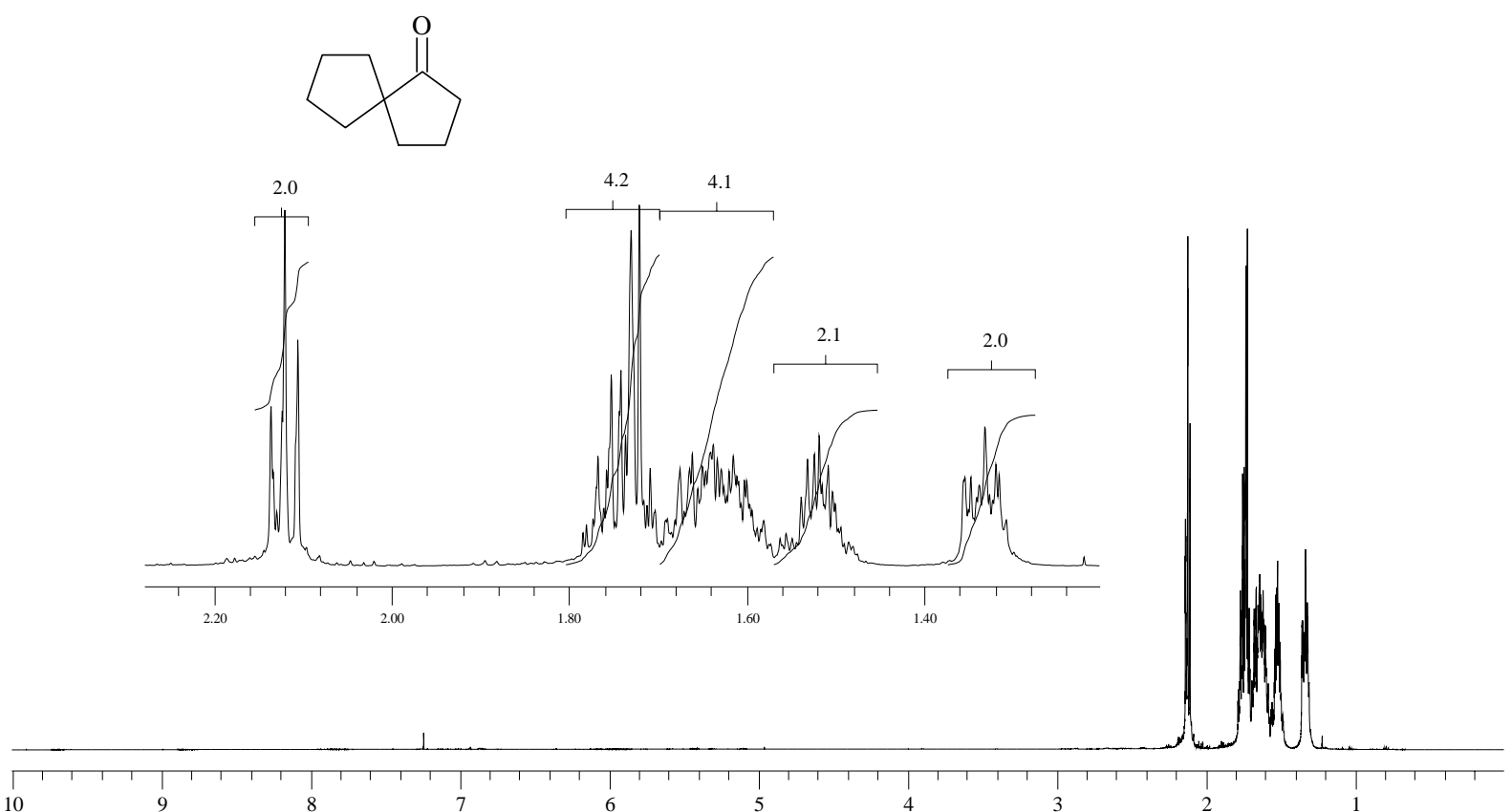

Abb. $75 \quad{ }^{1}$ H-NMR-Spektrum von Spiro[4.4]nonan-1-on (71)<smiles>O=C(CC1CCCC12CCCC2)C1CCCC1</smiles>
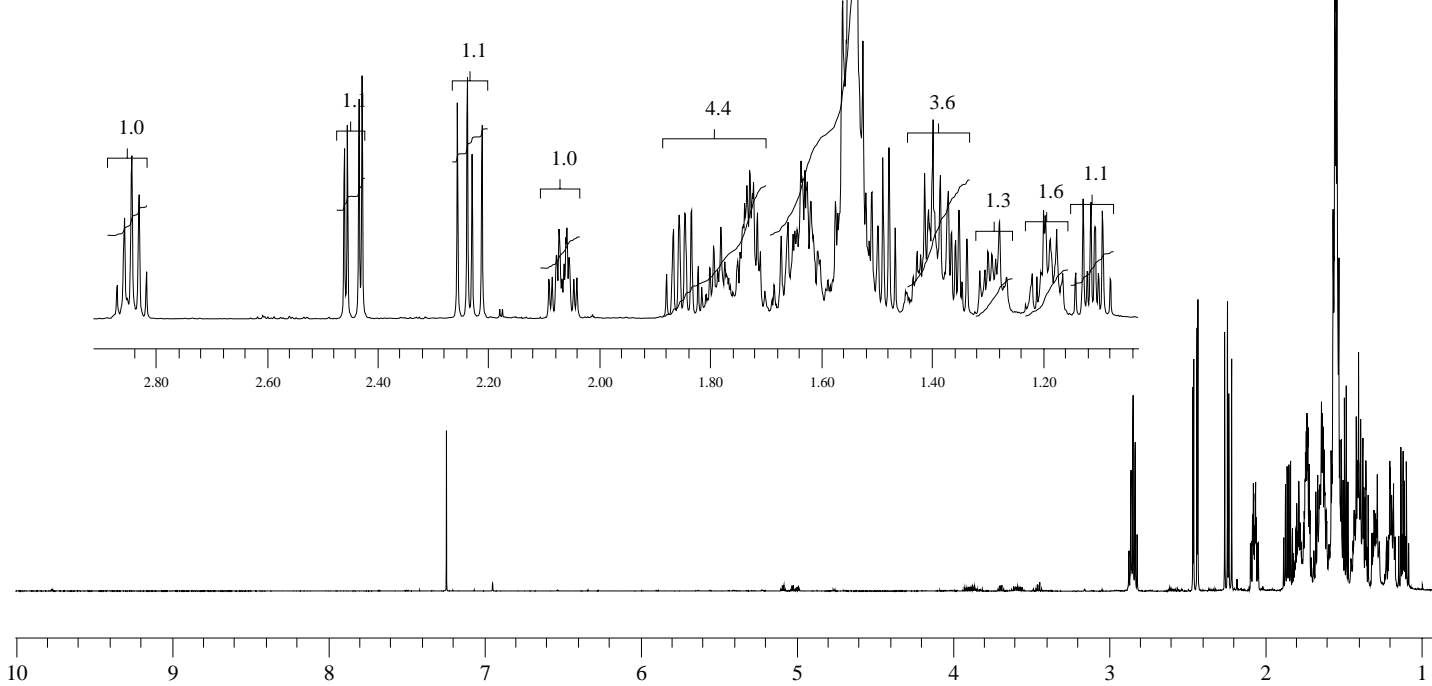

Abb. $76 \quad{ }^{1}$ H-NMR-Spektrum von 1-Cyclopentyl-2-spiro[4.4]non-1-yl-ethanon (75) 


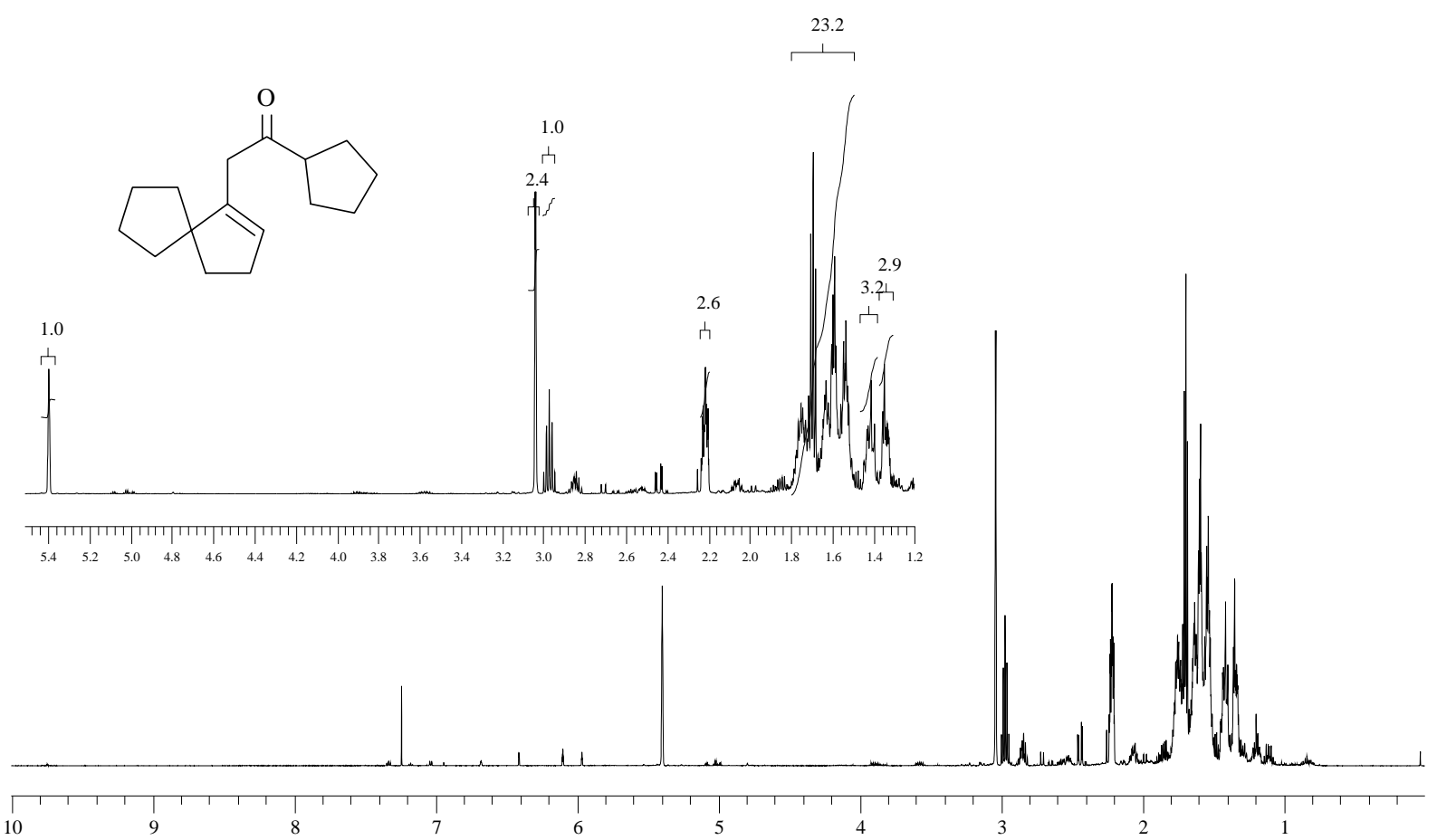

Abb. 77

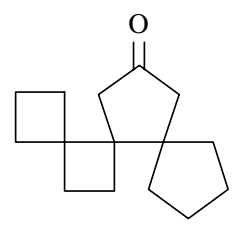

14.4

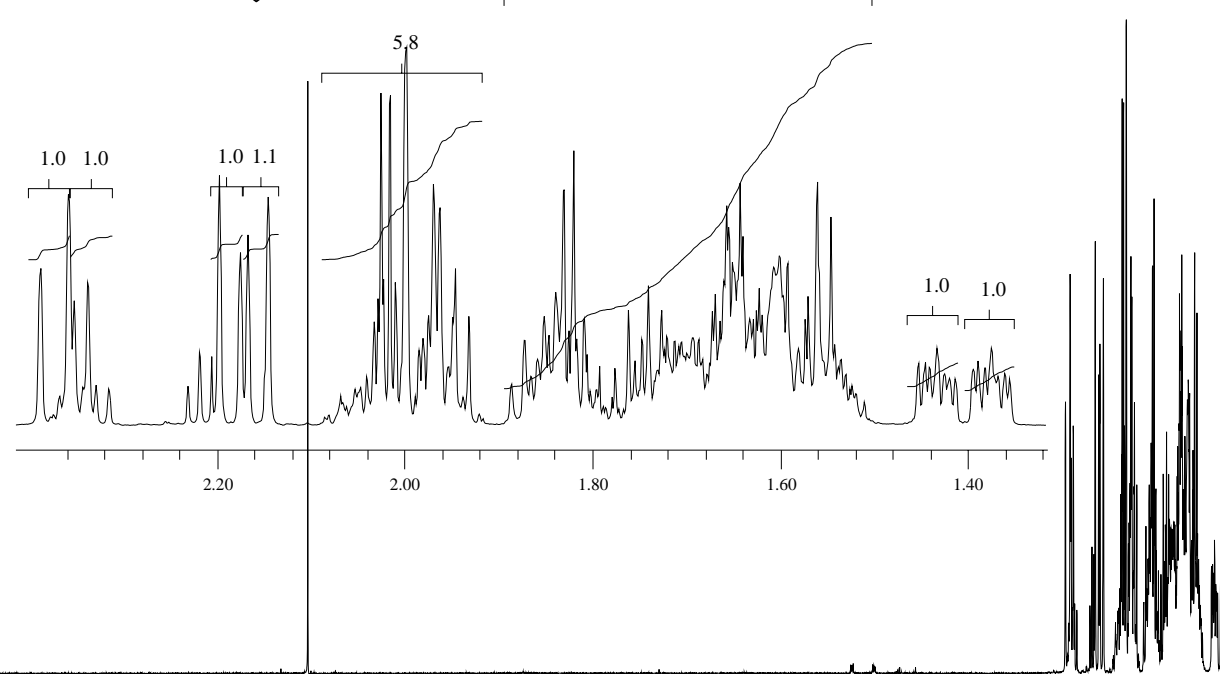

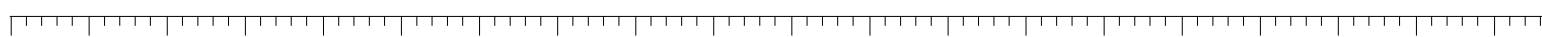

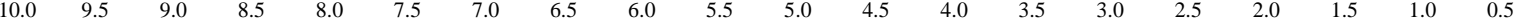

Abb. $78 \quad{ }^{1} \mathrm{H}-\mathrm{NMR}-S p e k t r u m$ von (5R*)-Trispiro[3.0.0.4.3.2]tetradecan-12-on (77) 


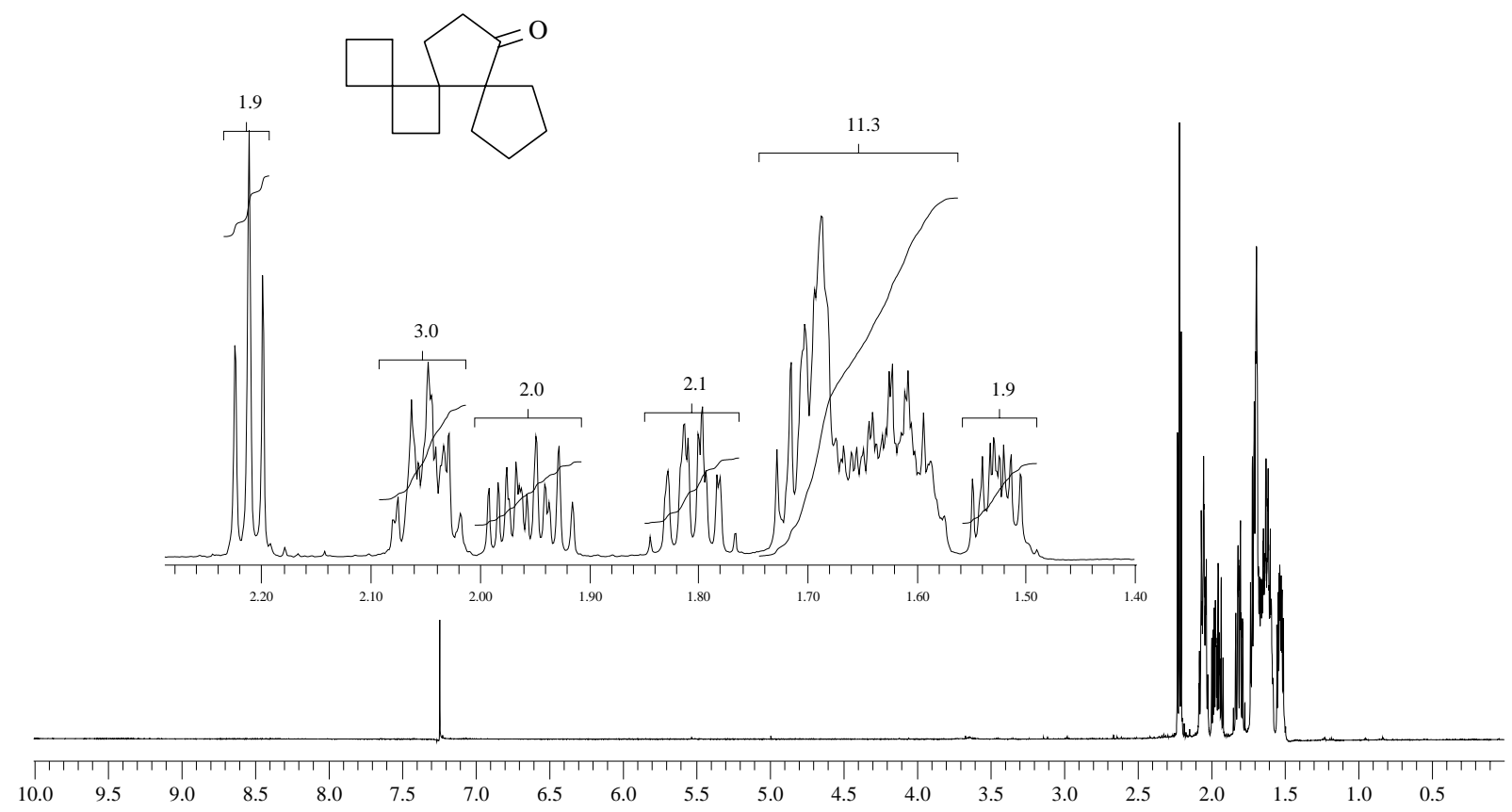

Abb. $79 \quad{ }^{1}$ H-NMR-Spektrum von (5R*)-Trispiro[3.0.0.4.3.2]tetradecan-11-on (78)
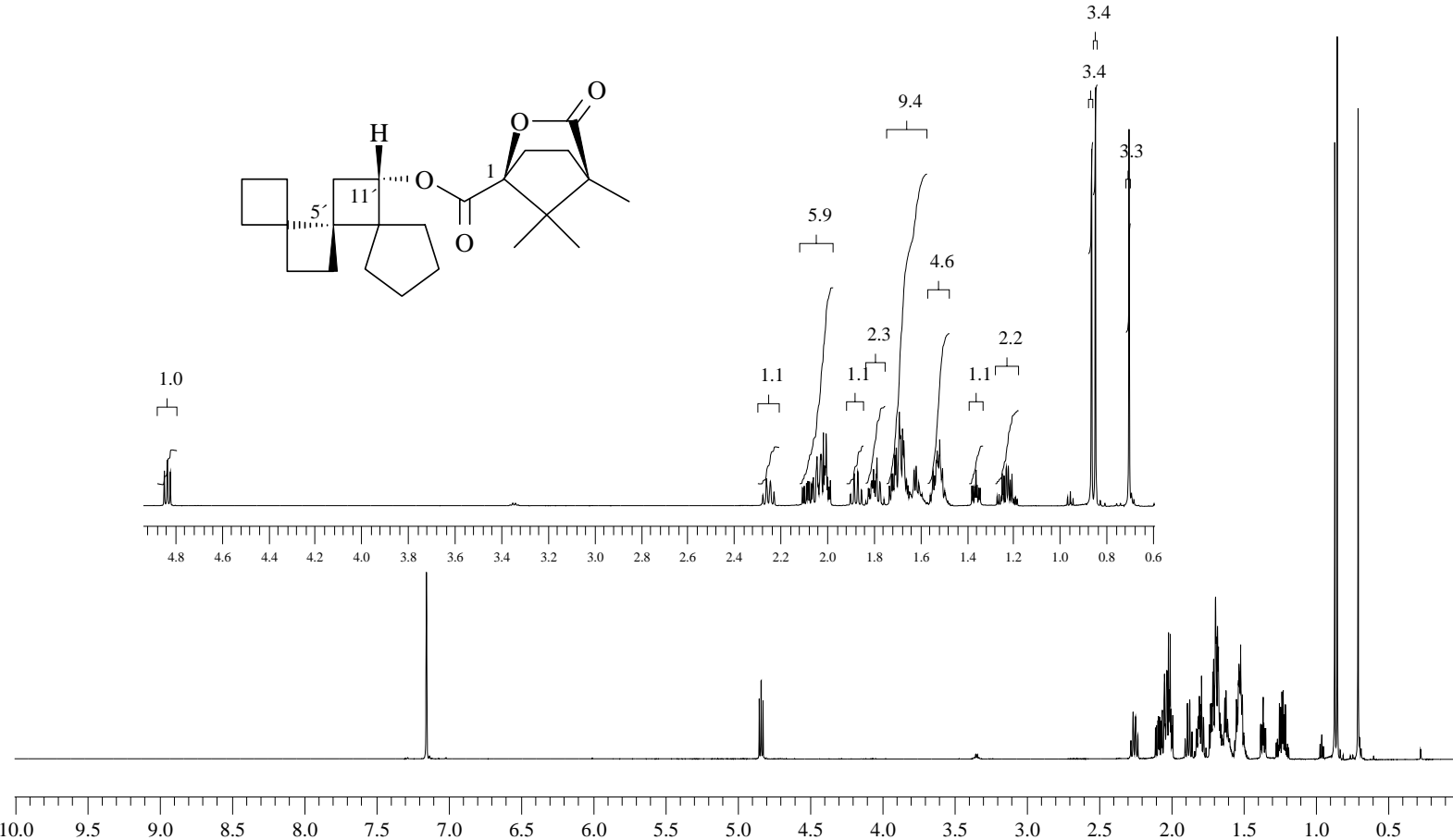
Abb. $80 \quad{ }^{1}$ H-NMR-Spektrum von (1S)-(+)-4,7,7-Trimethyl-3-oxo-2-oxa-bicyclo[2.2.1]heptan-1-carbonsäure(5'S,11'S)-trispiro[3.0.0.4.2.2] tetradecan-11-yl-ester $\left[\left(1 S, 5^{\prime} S, 11^{\prime} S\right)-(+)-79\right]$ 


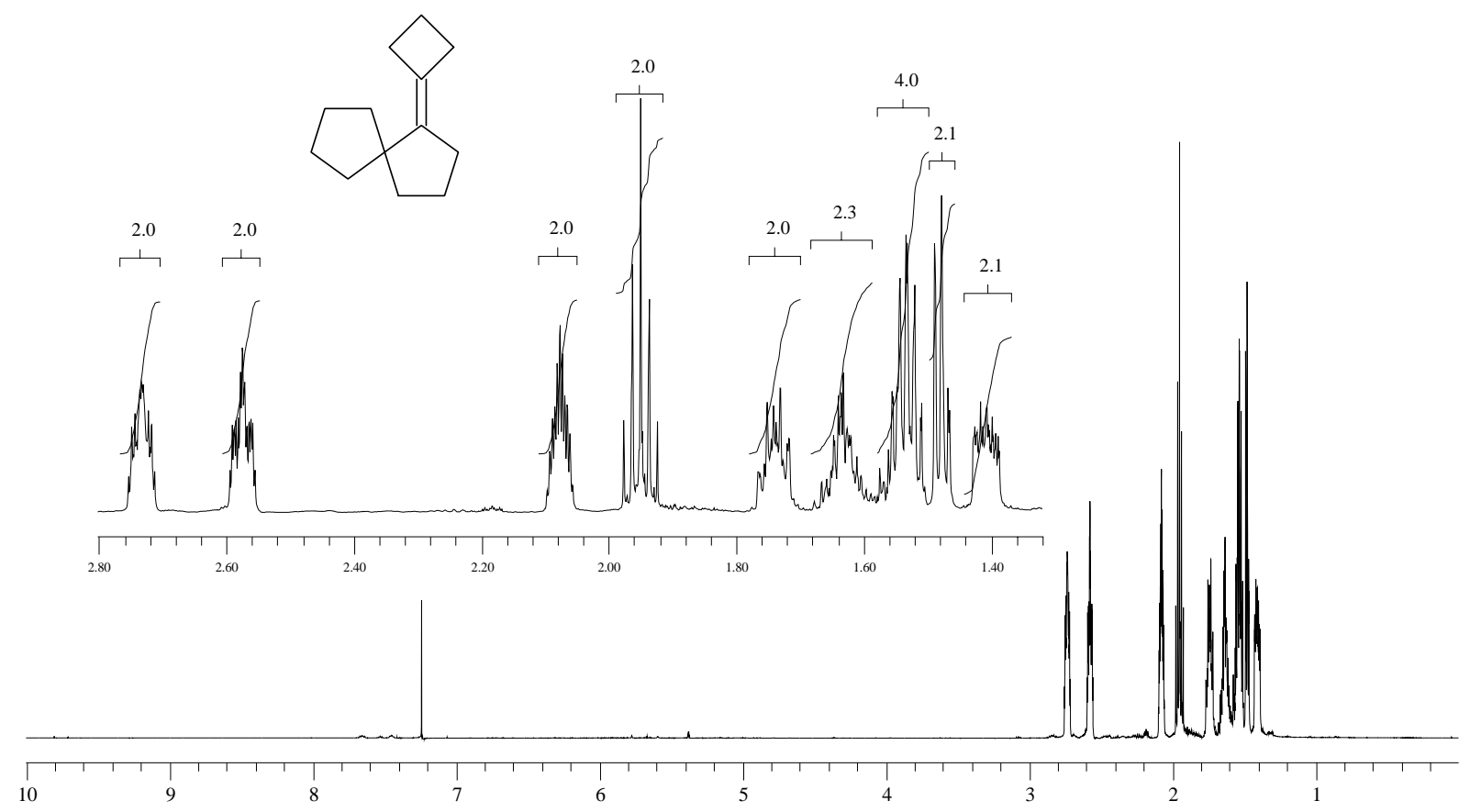

Abb. $81 \quad{ }^{1}$ H-NMR-Spektrum von Cyclobutyliden-spiro[4.4]nonan (85)

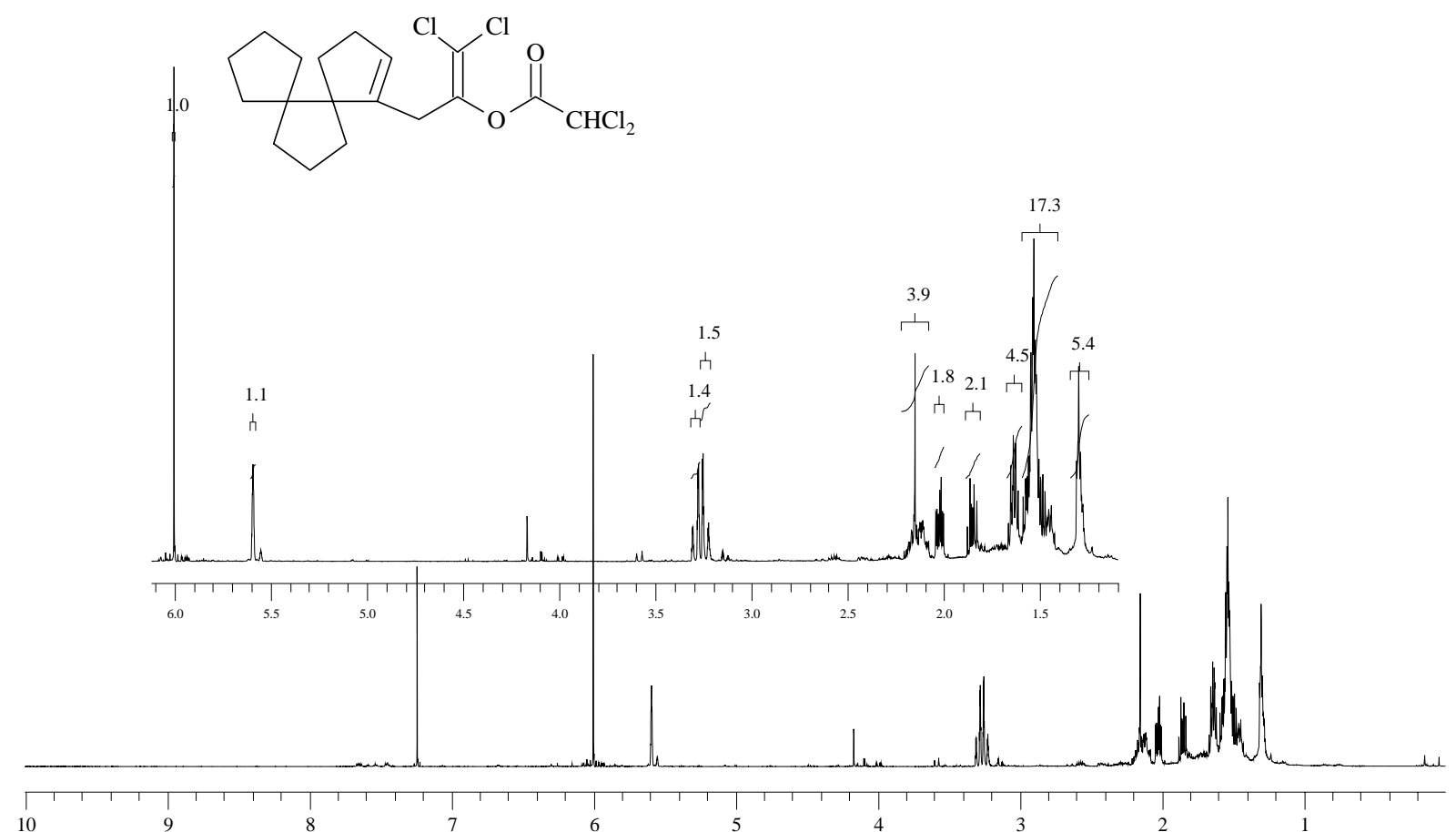

Abb. 82 1'H-NMR-Spektrum von Dichloressigsäure-2,2-dichlor-1-dispiro[4.0.4.3]tridec1-en-1-yl-methyl-vinyl-ester (94) 

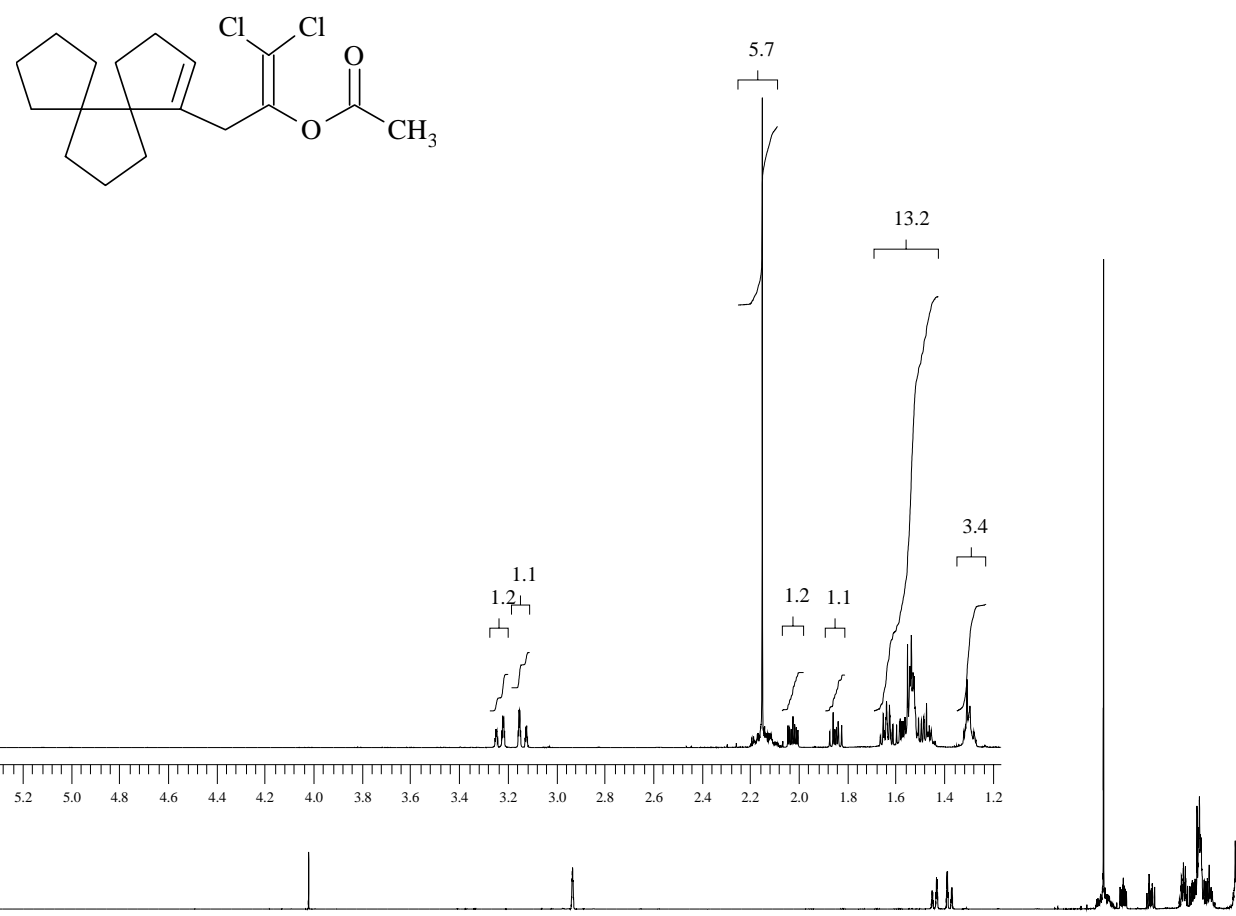

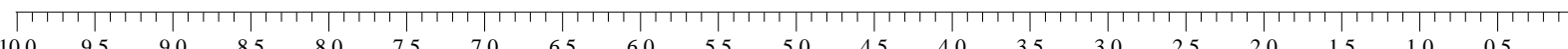
Abb. $83 \quad{ }^{1}$ H-NMR-Spektrum von Essigsäure-2,2-dichlor-1-dispiro[4.0.4.3]tridec-1-en-1yl-methyl-vinyl-ester (95)
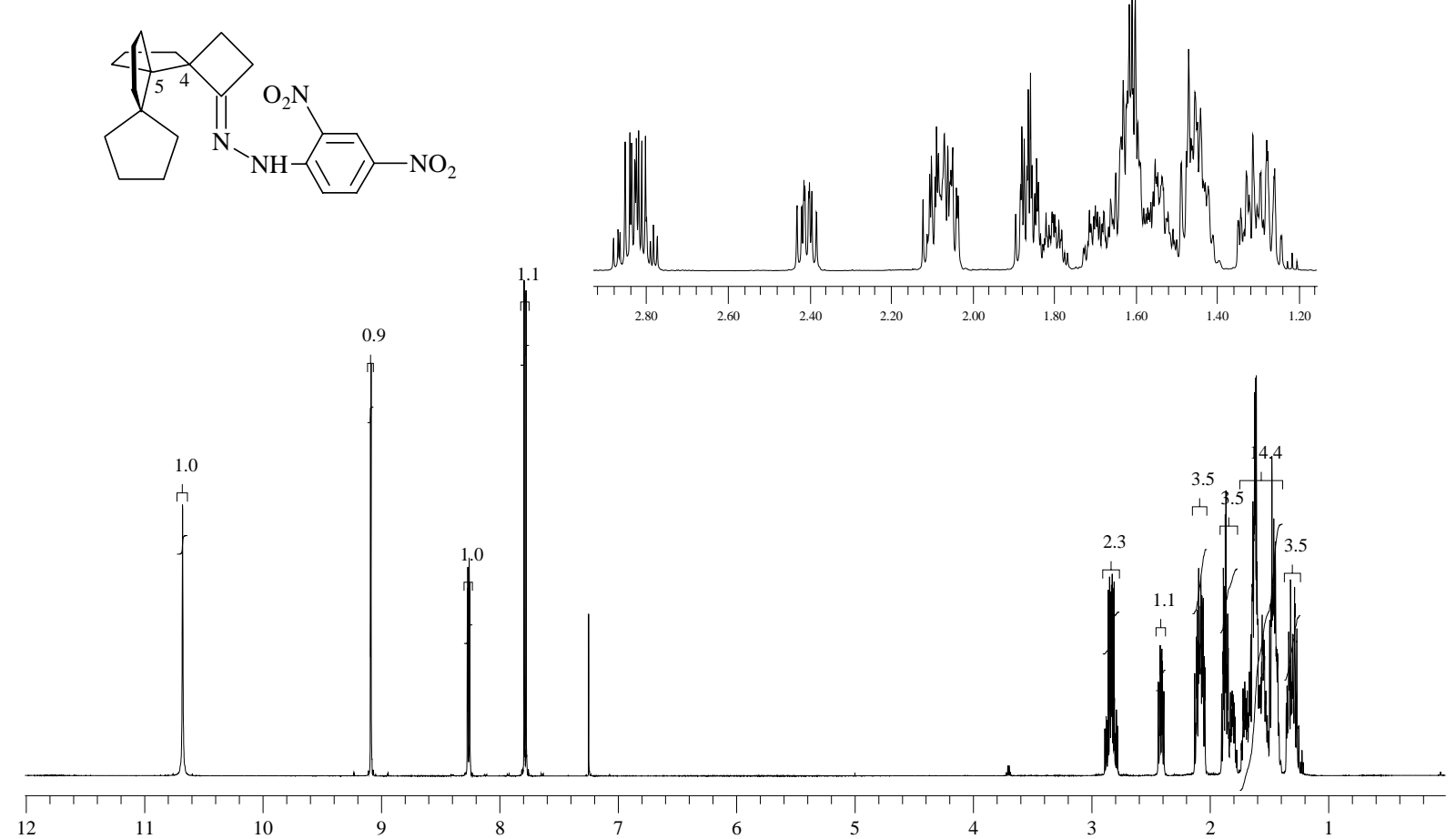

Abb. $84 \quad{ }^{1} \mathrm{H}$-NMR-Spektrum von $\left(4 R^{*}, 5 S^{*}\right)$-N-Trispiro[3.0.0.4.3.3] $\mathrm{N}^{\prime}$-2,4-dinitrophenyl-hydrazin [(4R*,5S*)-98] 


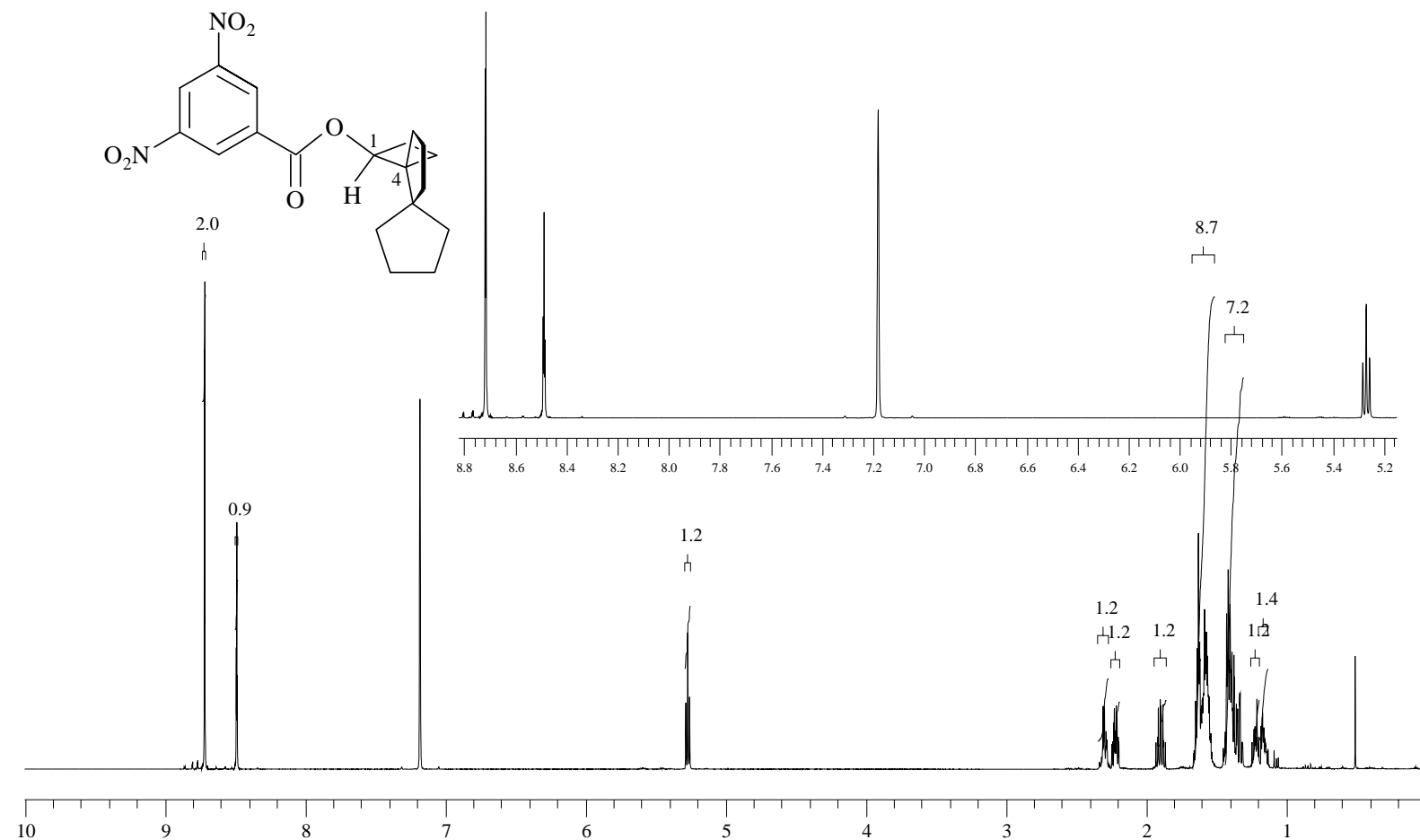

Abb. $85 \quad{ }^{1}$ H-NMR-Spektrum von (1S,4R)-(+)-3,5-Dinitrobenzoesäure-dispiro[3.0.4.3]dodec-1-yl-ester [(1S,4R)-(+)-104] 


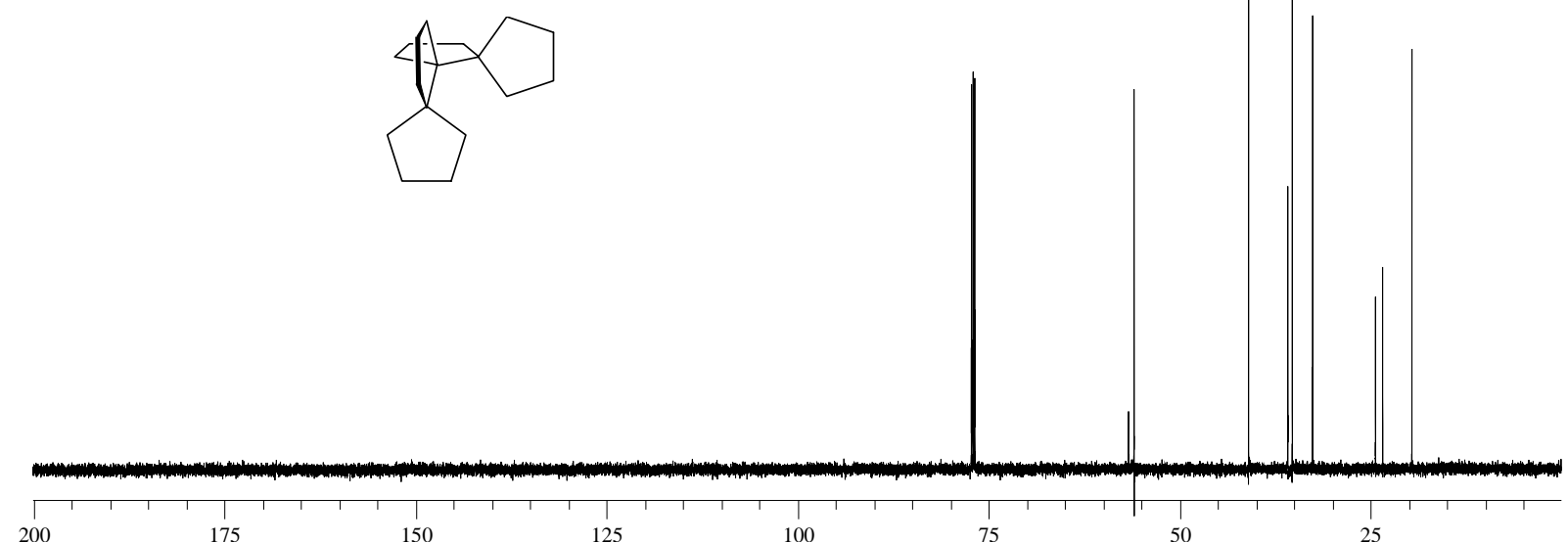

Abb. $86 \quad{ }^{13}$ C-NMR-Spektrum von Trispiro[4.0.0.4.3.3]heptadecan (8)

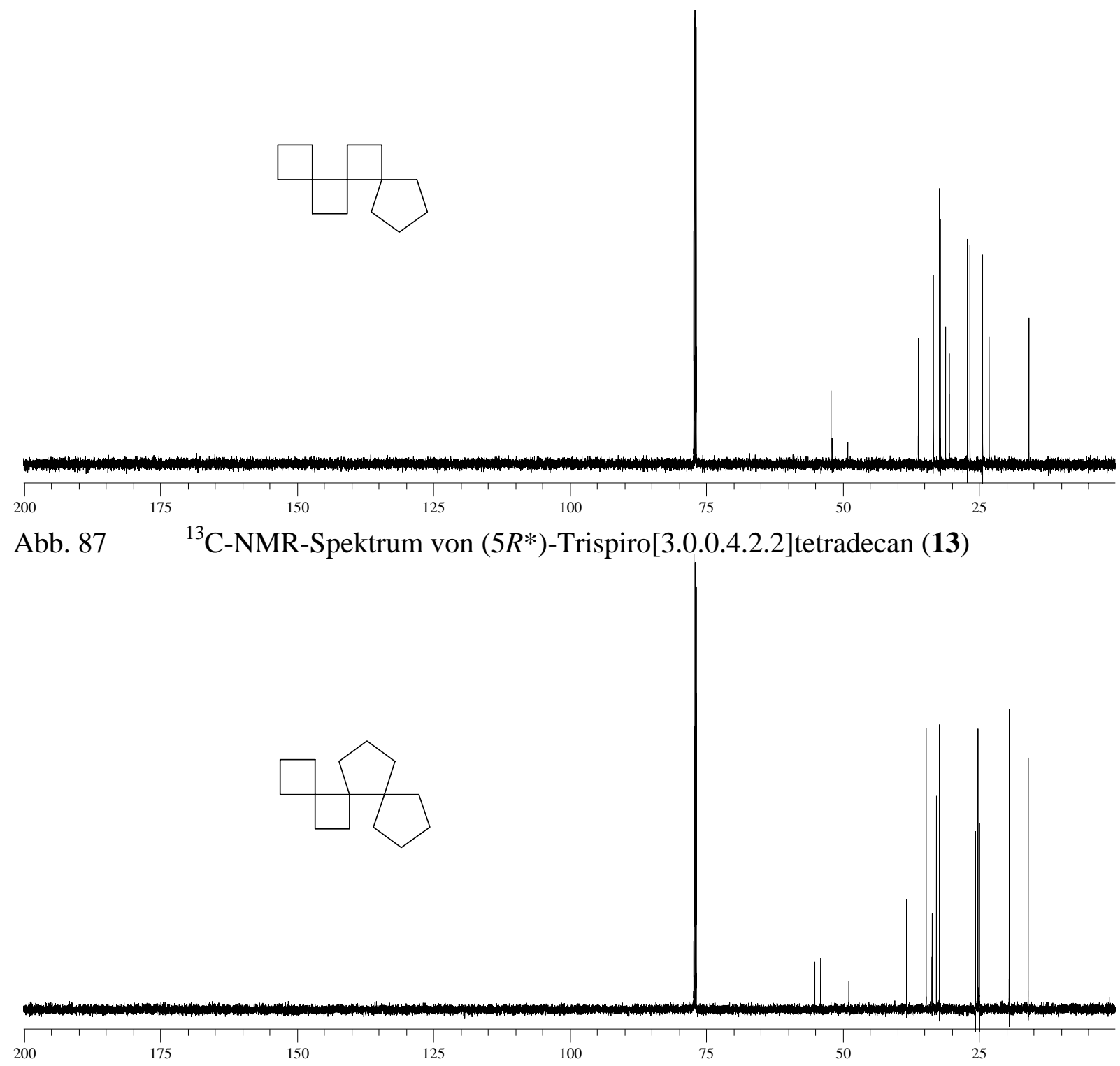

Abb. $88 \quad{ }^{13}$ C-NMR-Spektrum von (5R*)-Trispiro[3.0.0.4.3.2]pentadecan (14) 

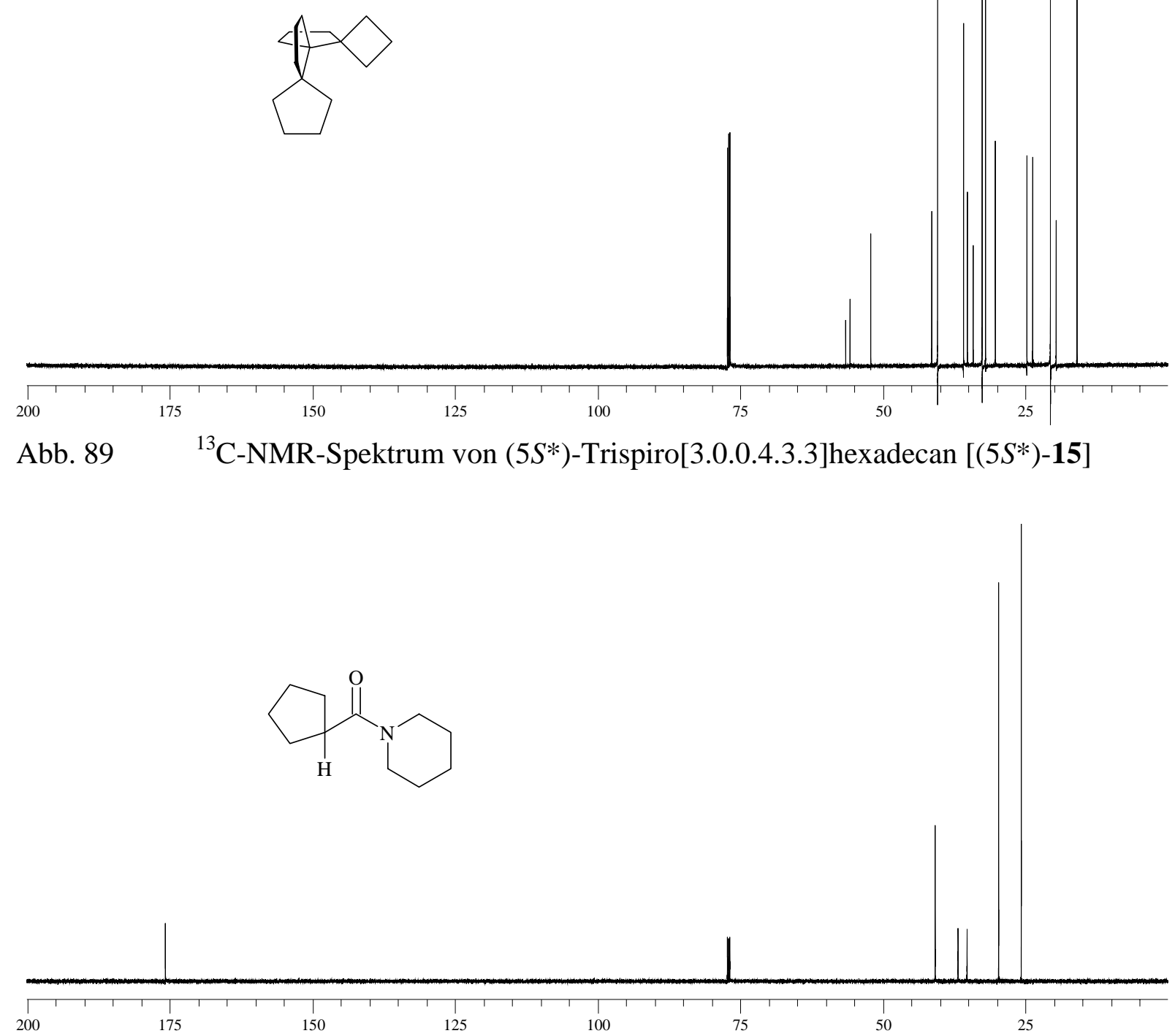

Abb. $90 \quad{ }^{13}$ C-NMR-Spektrum von 1-(Cyclopentylcarbonyl)-piperidin (23)
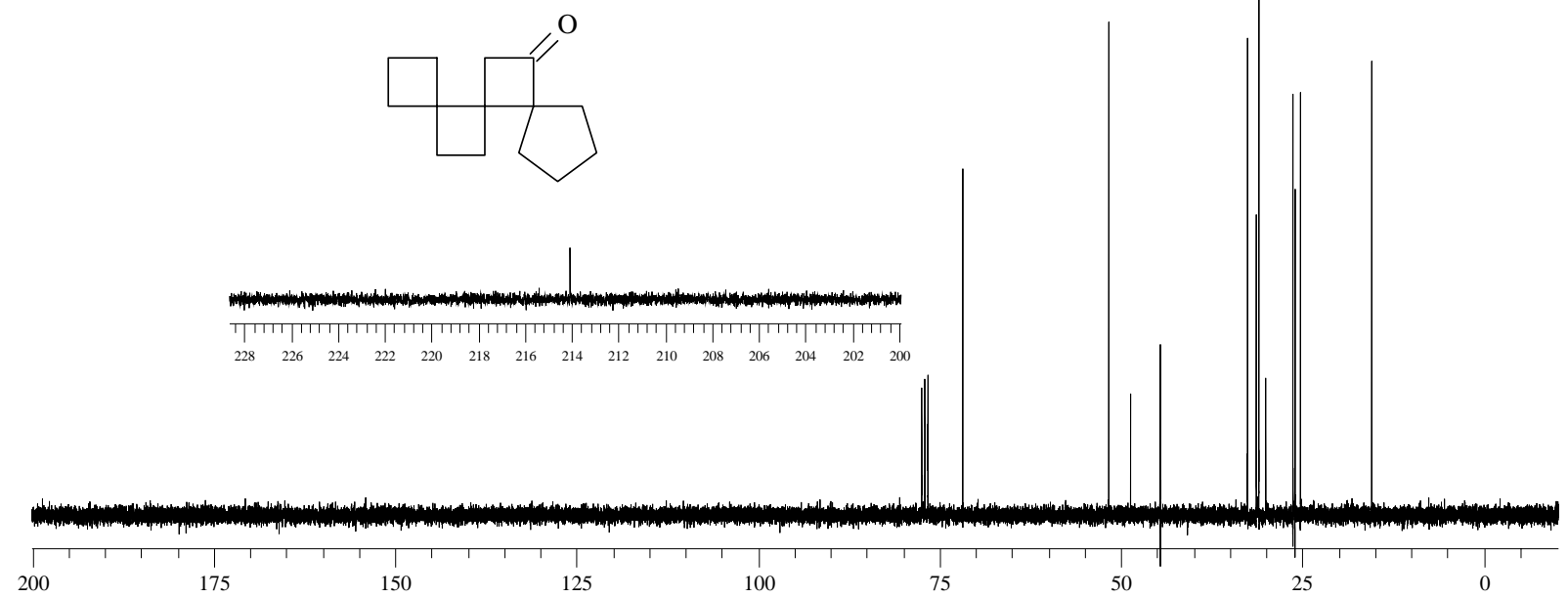

Abb. $91 \quad{ }^{13}$ C-NMR-Spektrum von (5R*)-Trispiro[3.0.0.4.2.2]tetradecan-11-on (24) 


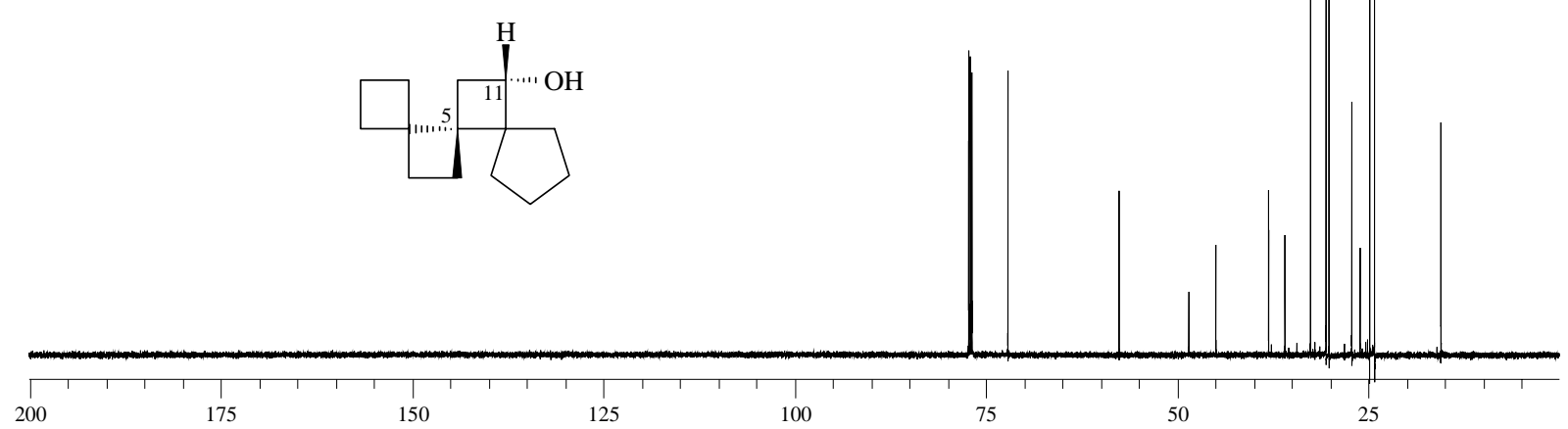

Abb. $92 \quad{ }^{13}$ C-NMR-Spektrum von (5S,11S)-(+)-Trispiro[3.0.0.4.2.2]tetradecan-11-ol [(5S,11S)-(+)-28)]
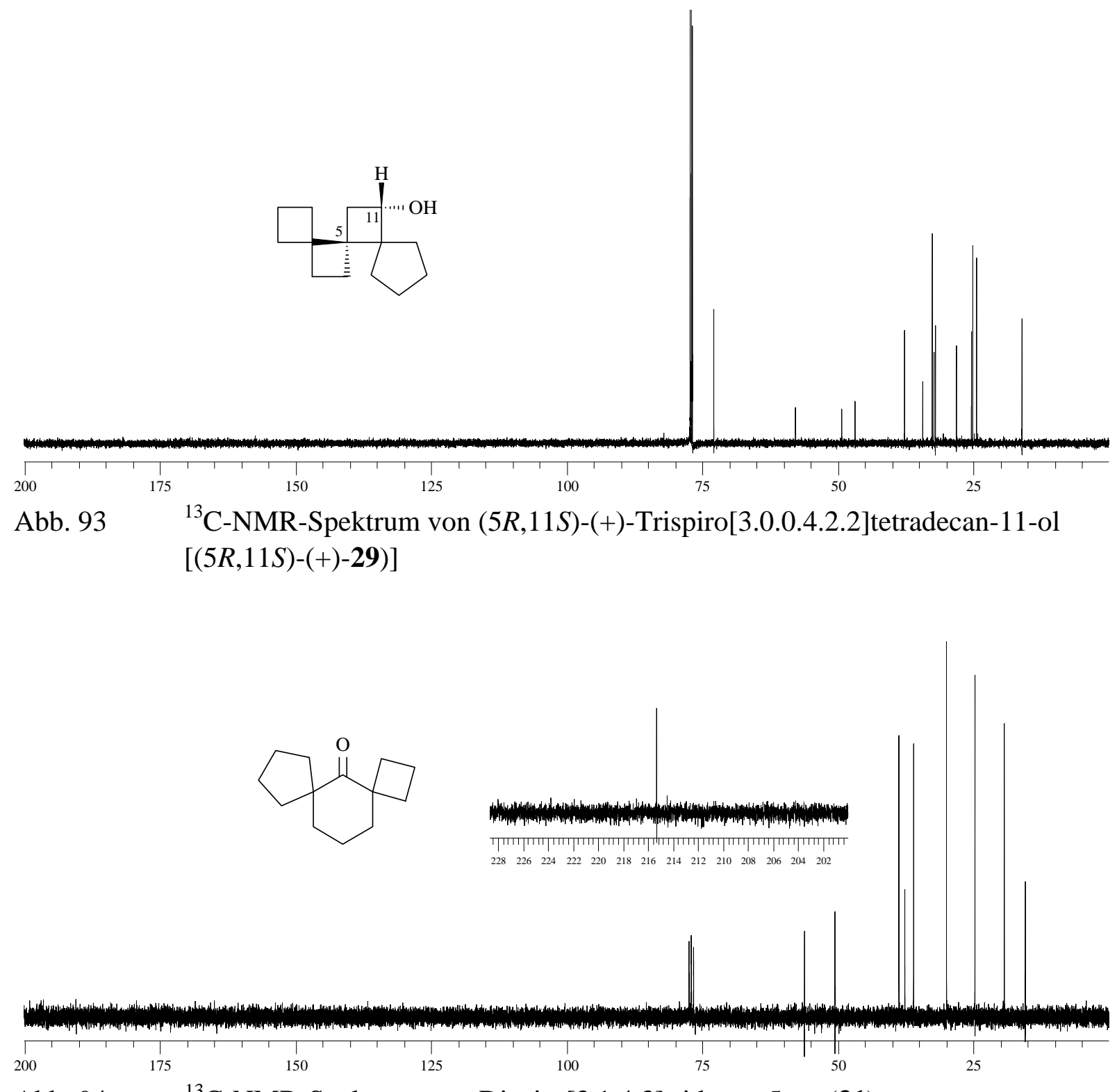

Abb. $94 \quad{ }^{13}$ C-NMR-Spektrum von Dispiro[3.1.4.3]tridecan-5-on (31) 


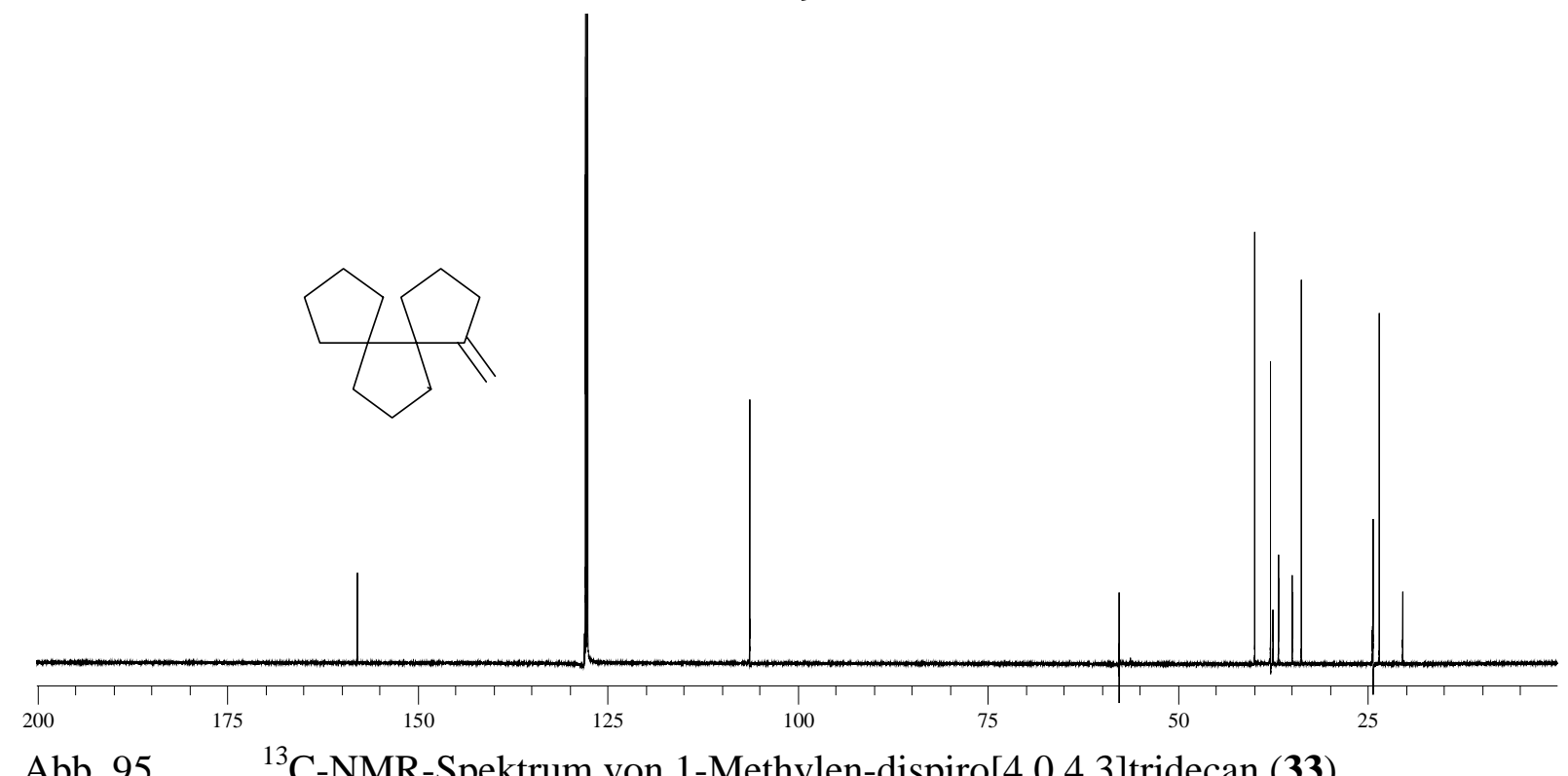

Abb. $95 \quad{ }^{13}$ C-NMR-Spektrum von 1-Methylen-dispiro[4.0.4.3]tridecan (33)

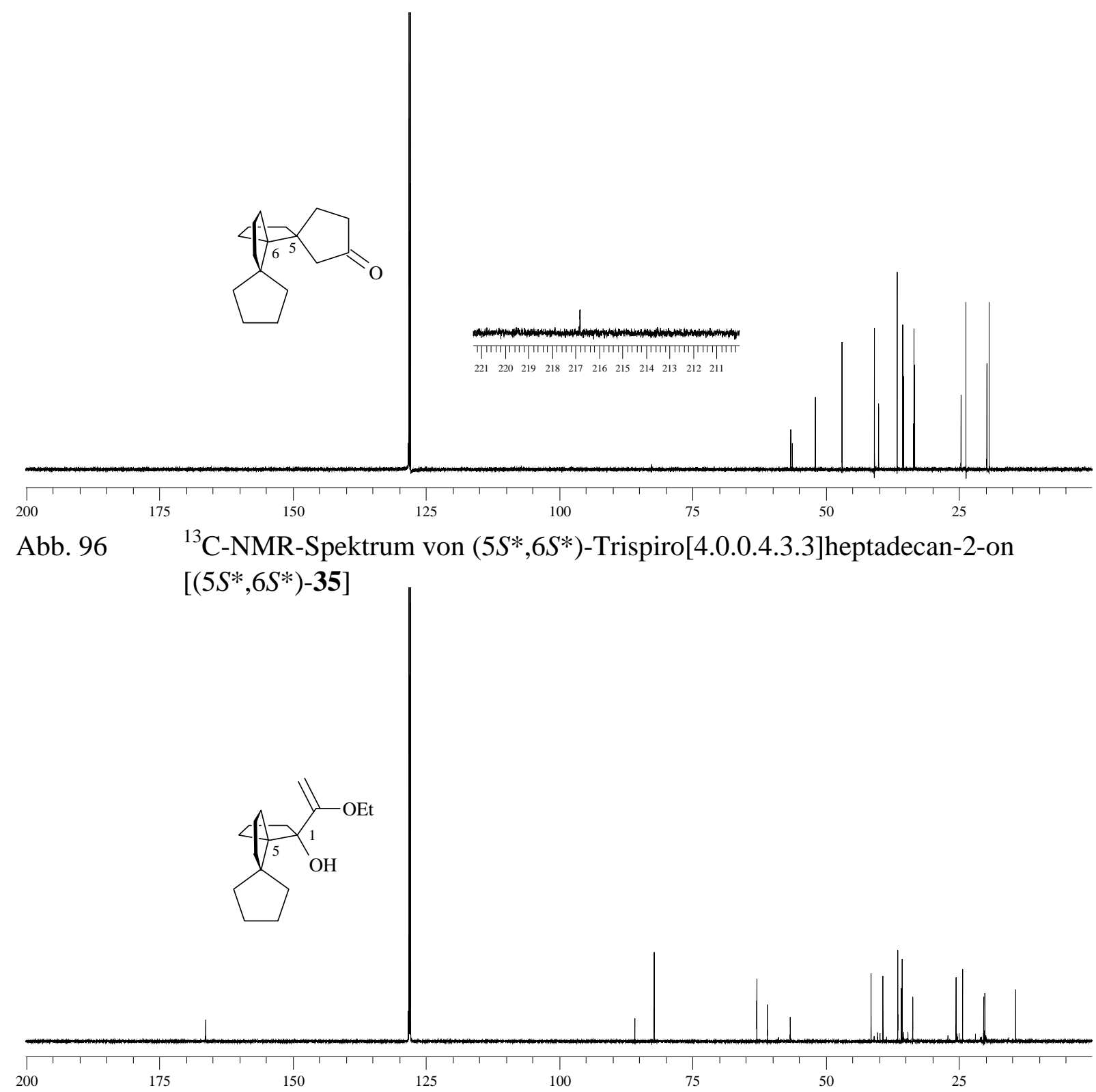

Abb. $97 \quad{ }^{13} \mathrm{C}-\mathrm{NMR}-S p e k t r u m$ von $\left(1 S^{*}, 5 S^{*}\right)$-1-(1-Ethoxy-vinyl)-dispiro[4.0.4.3]tridecan-1-ol [(1S*,5S*)-41] 

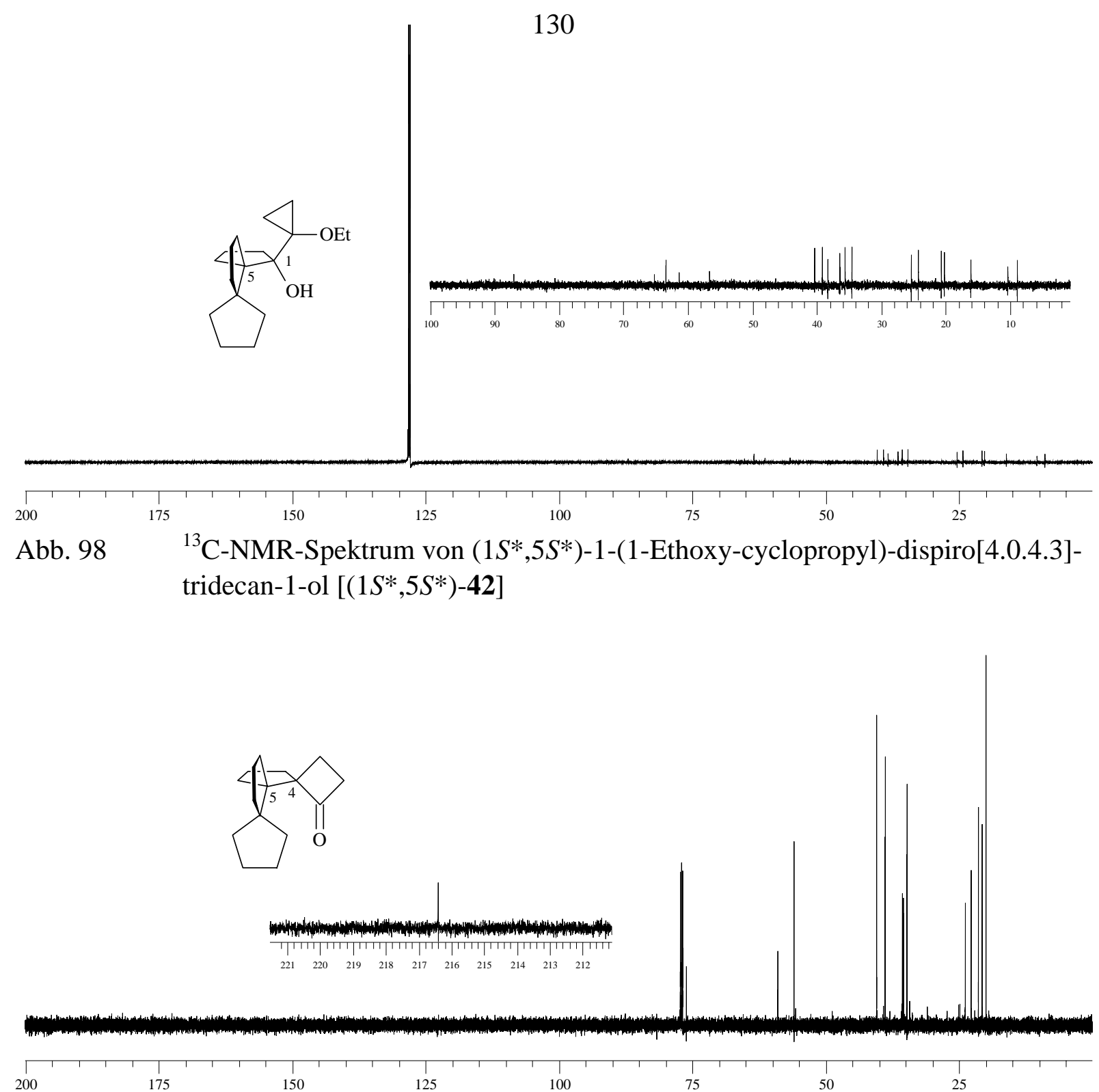

Abb. $99 \quad{ }^{13}$ C-NMR-Spektrum von $\left(4 R^{*}, 5 S^{*}\right)$-Trispiro[3.0.0.4.3.3] hexadecan-1-on [(4R*,5S*)-43]

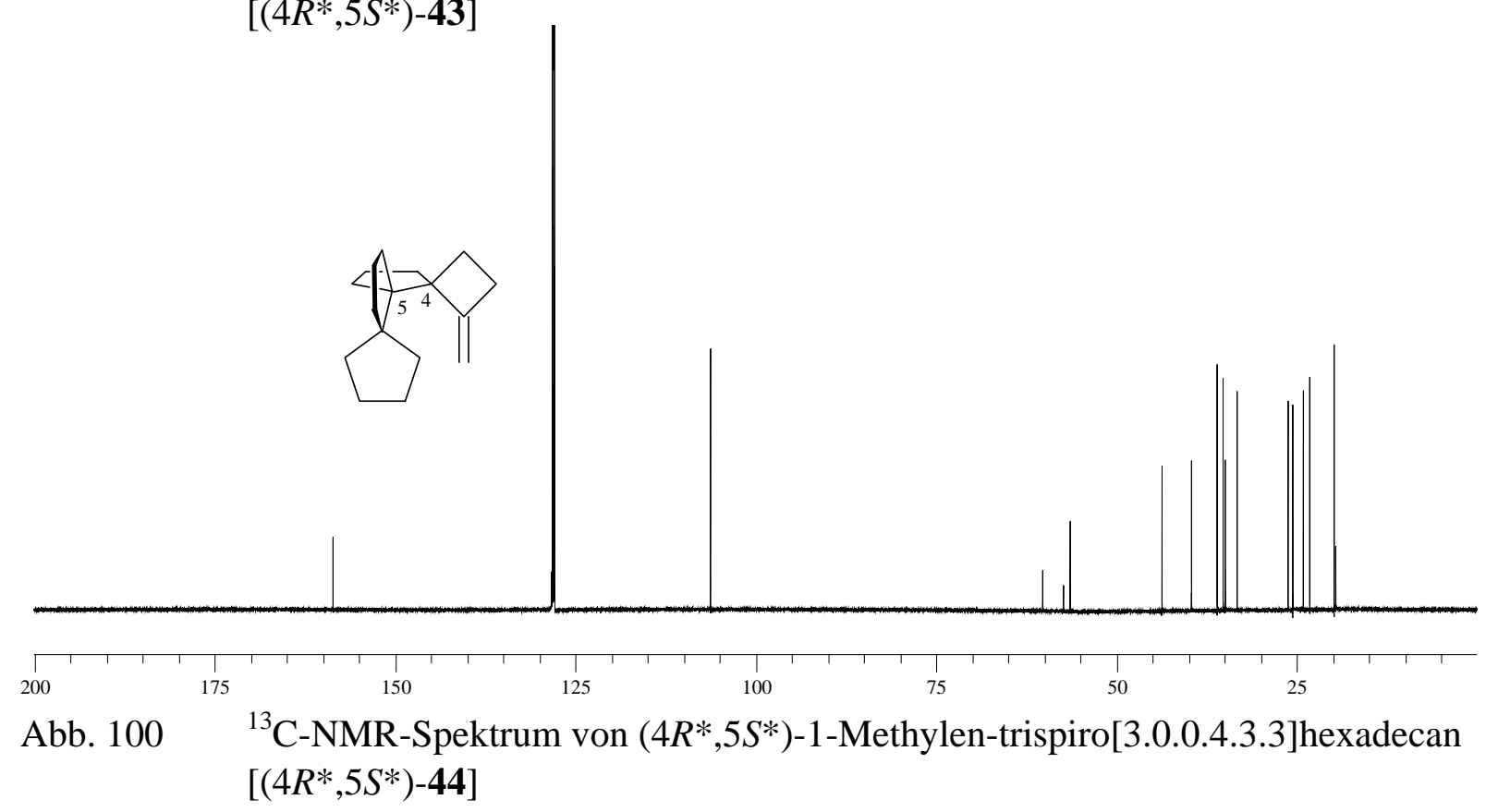



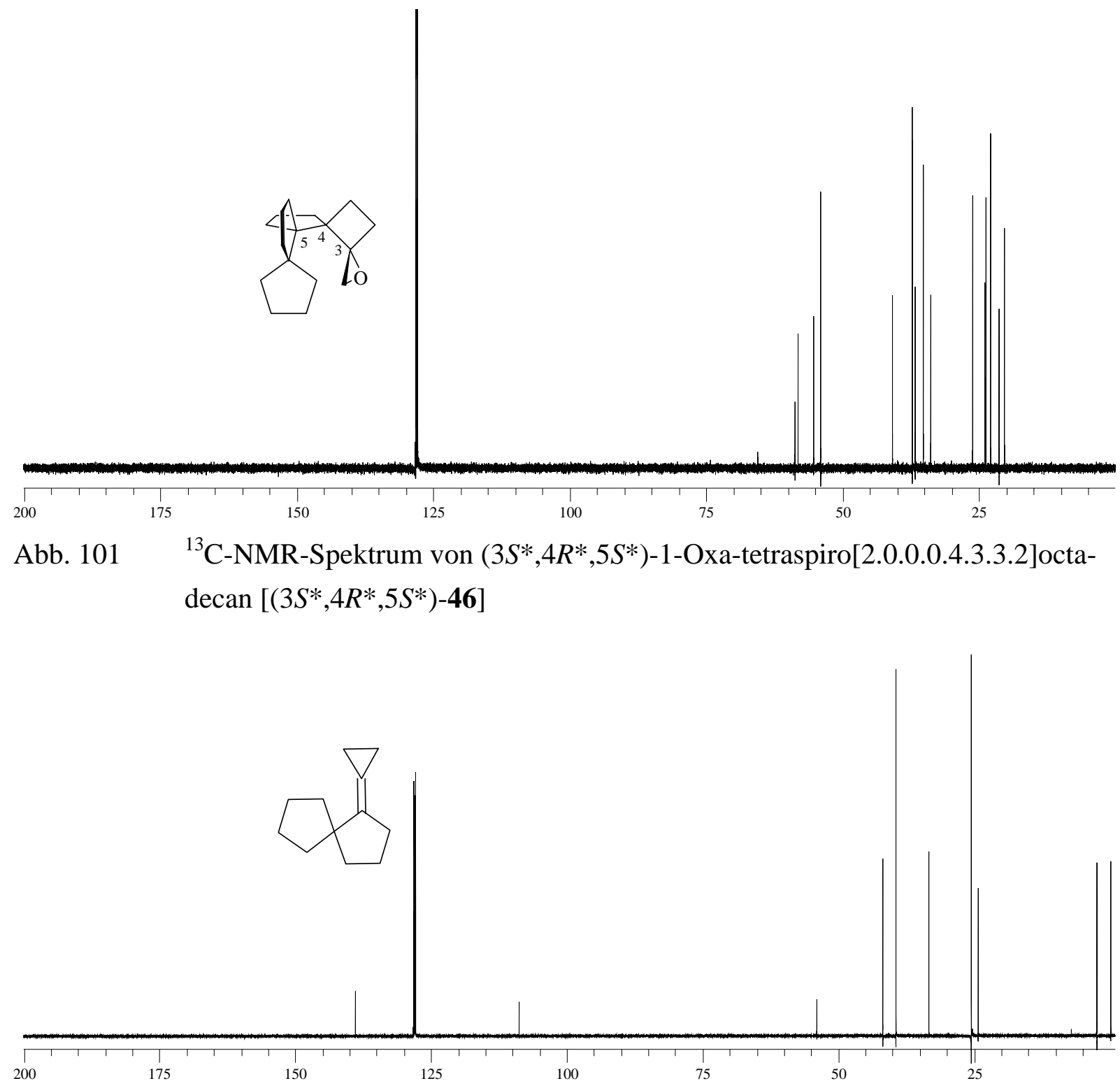

Abb. $102 \quad{ }^{13}$ C-NMR-Spektrum von Cyclopropyliden-spiro[4.4]nonan (49)

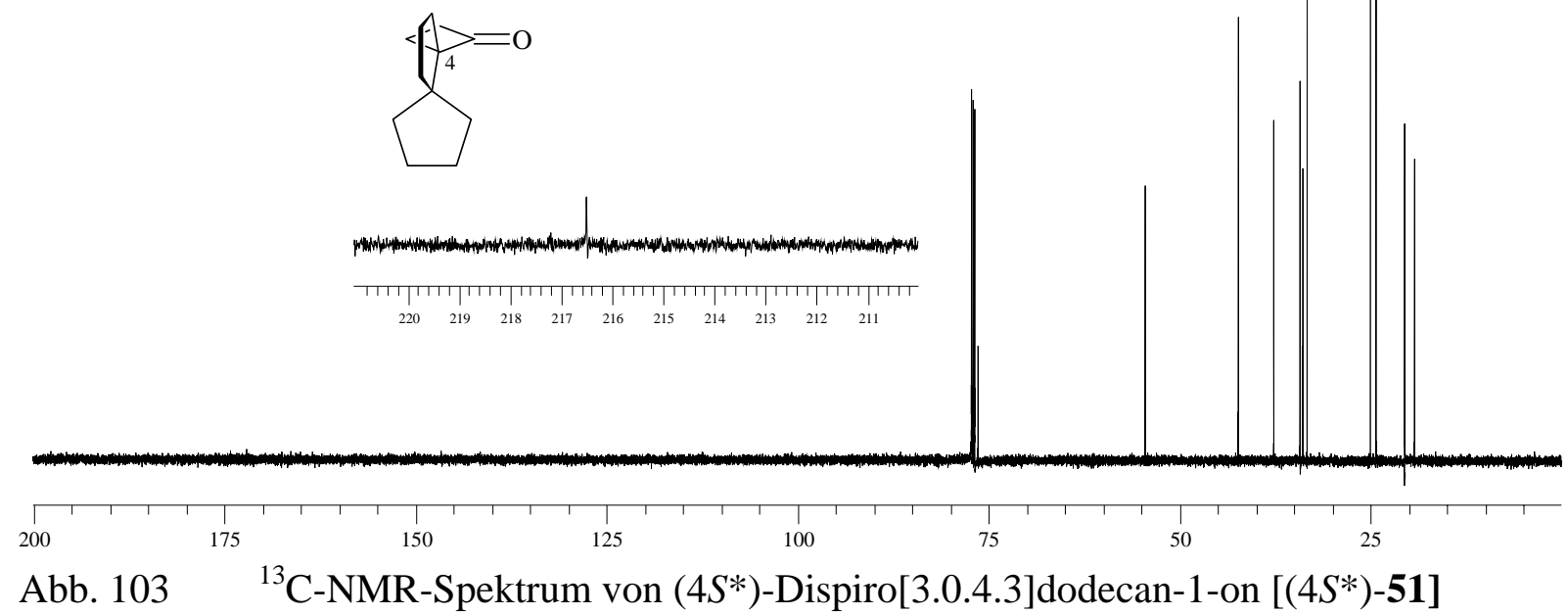




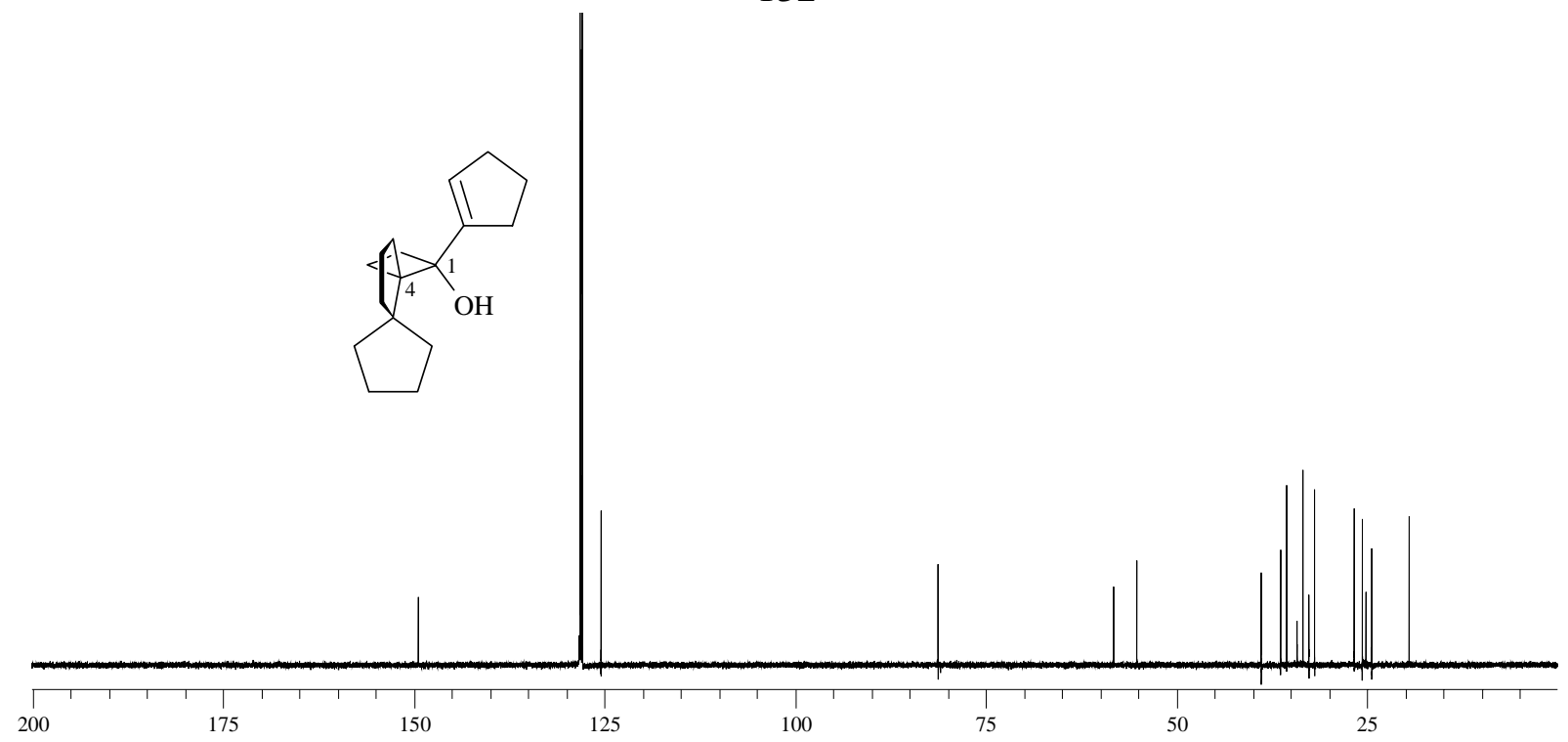

Abb. $104 \quad{ }^{13}$ C-NMR-Spektrum von $\left(1 R^{*}, 4 S^{*}\right)-1-C y c l o p e n t-1-e n y l-d i s p i r o[3.0 .4 .3]$ dodecan-1-ol $\left[\left(1 R^{*}, 4 S^{*}\right)-52\right]$

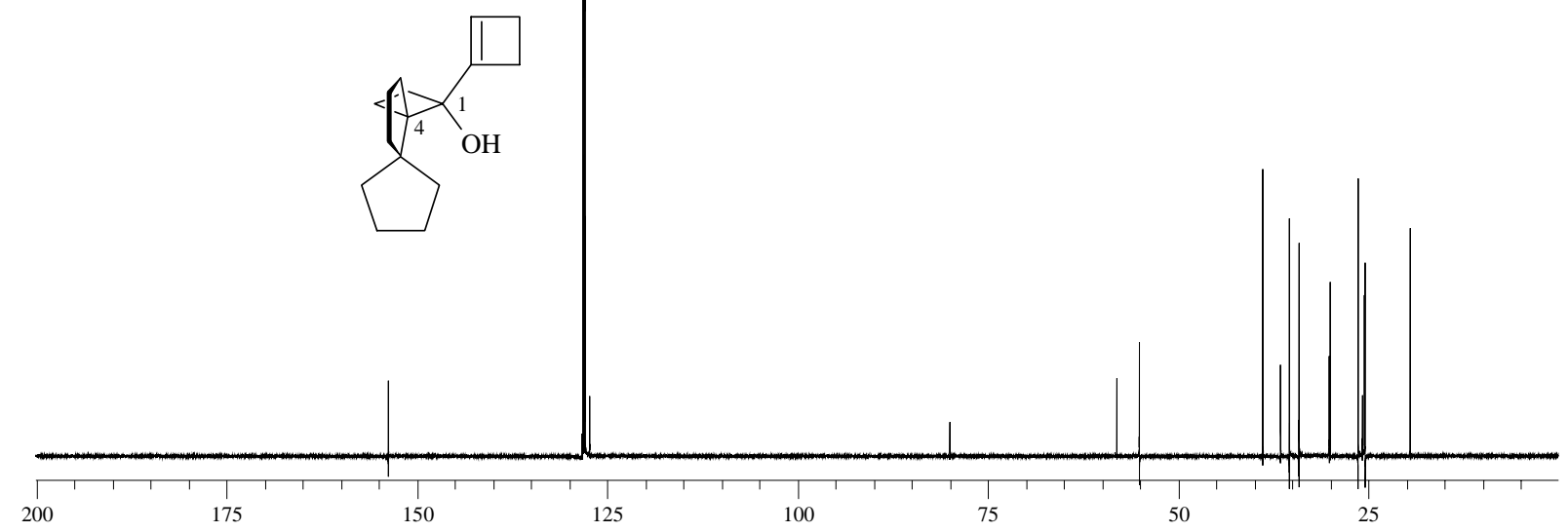

Abb. $105 \quad{ }^{13}$ C-NMR-Spektrum von $\left(1 R^{*}, 4 S^{*}\right)-1-C y c l o b u t-1-e n y l-d i s p i r o[3.0 .4 .3] d o-$

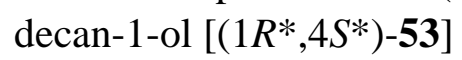

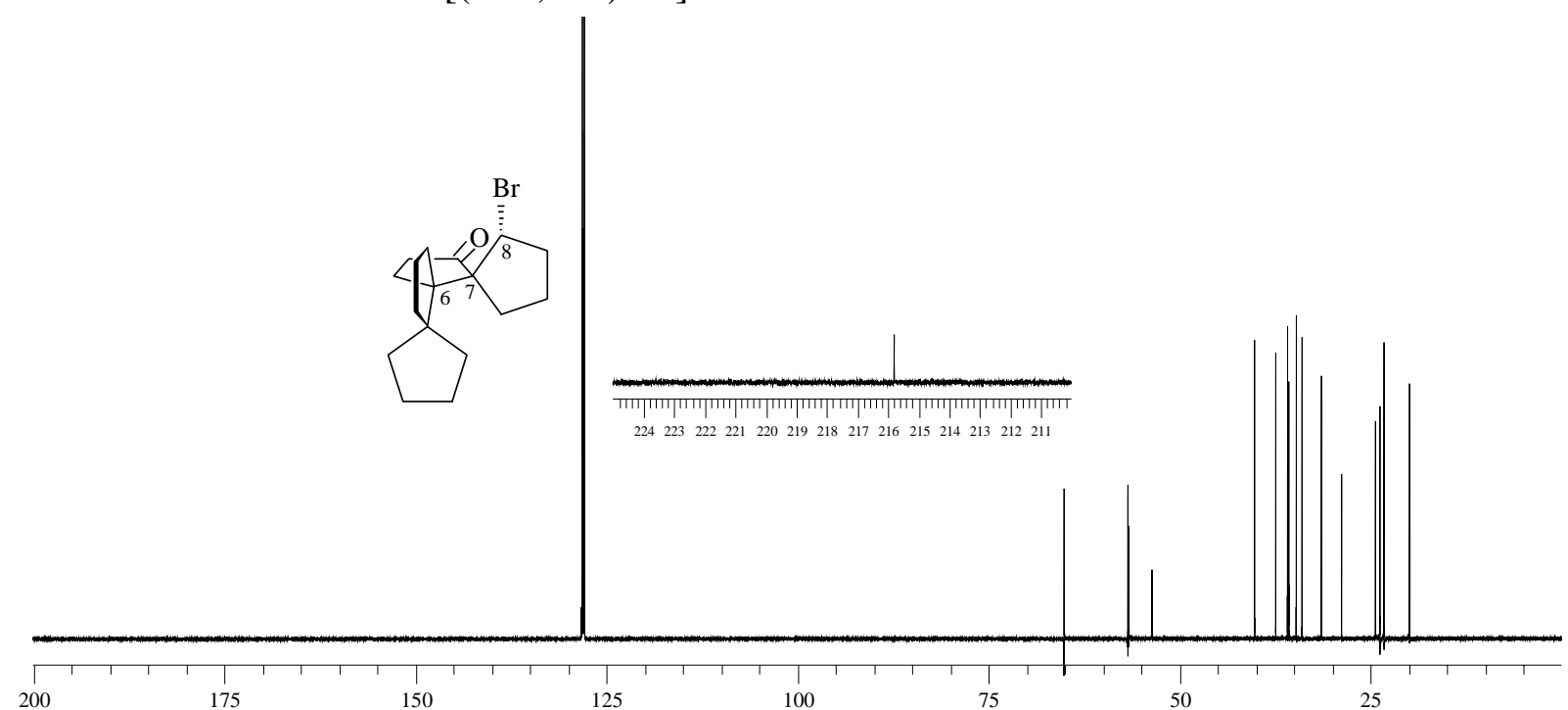

Abb. $106 \quad{ }^{13}$ C-NMR-Spektrum von $\left(6 S^{*}, 7 S^{*}, 8 R^{*}\right)$-8-Brom-trispiro[4.0.0.4.3.3] heptadecan-12-on [(6S*,7S*,8R*)-54] 


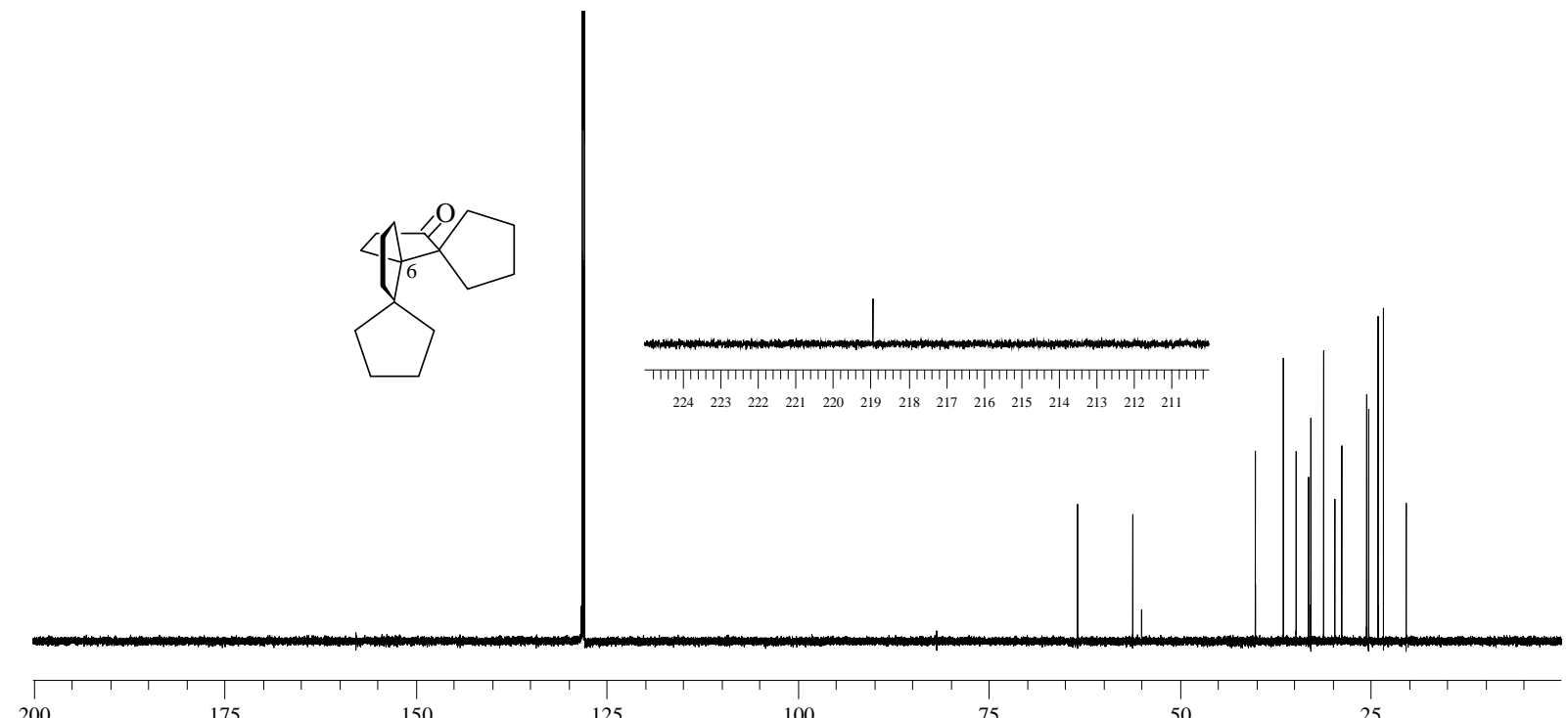

Abb. $107 \quad{ }^{13}$ C-NMR-Spektrum von (6S*)-Trispiro[4.0.0.4.3.3]heptadecan-12-on [(6S*)-

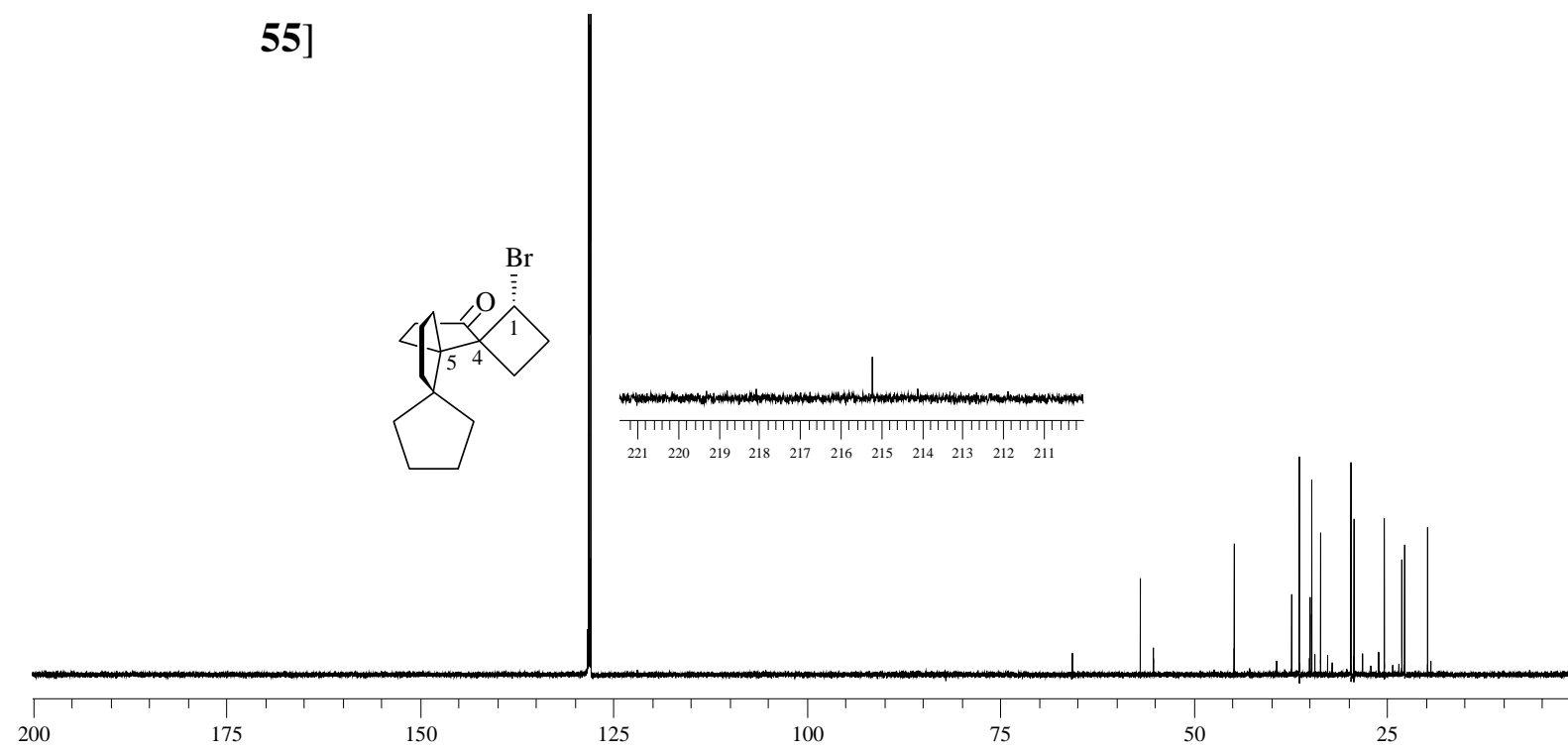

Abb. $108 \quad{ }^{13} \mathrm{C}-\mathrm{NMR}-\mathrm{Spektrum}$ von $\left(1 R^{*}, 4 S^{*}, 5 S^{*}\right)-1$-Brom-trispiro[3.0.0.4.3.3] hexadecan-16-on [(1R*,4S*,5S*)-56]

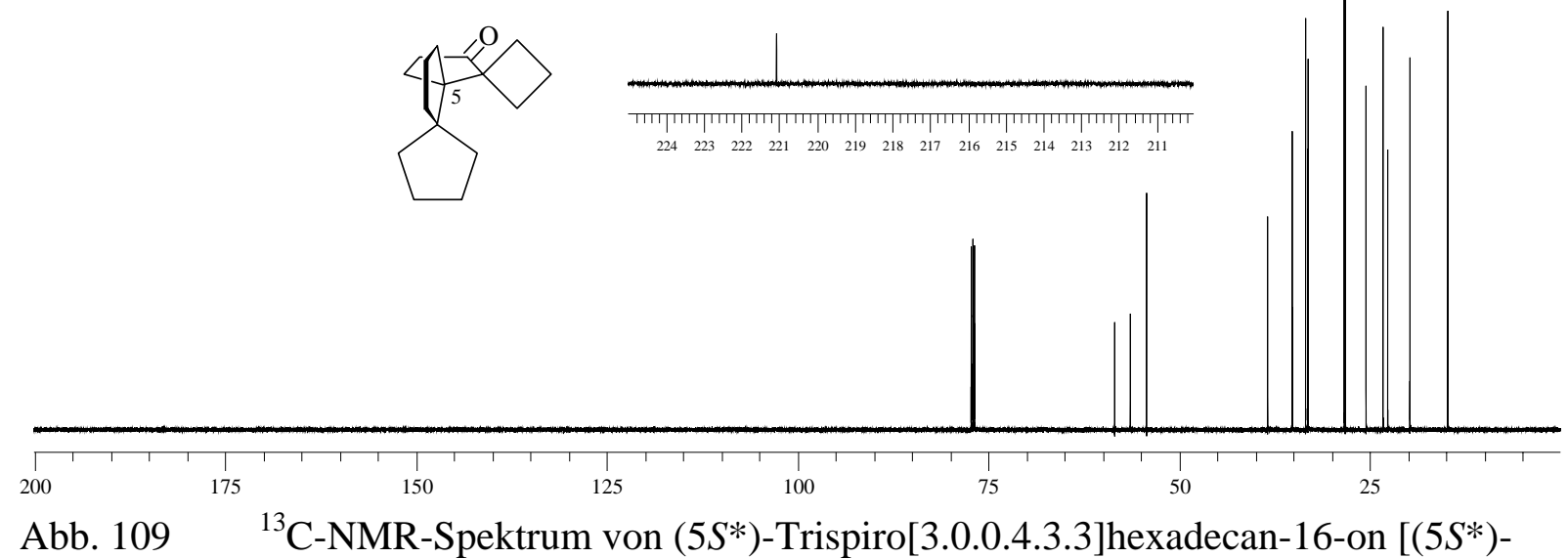
57] 


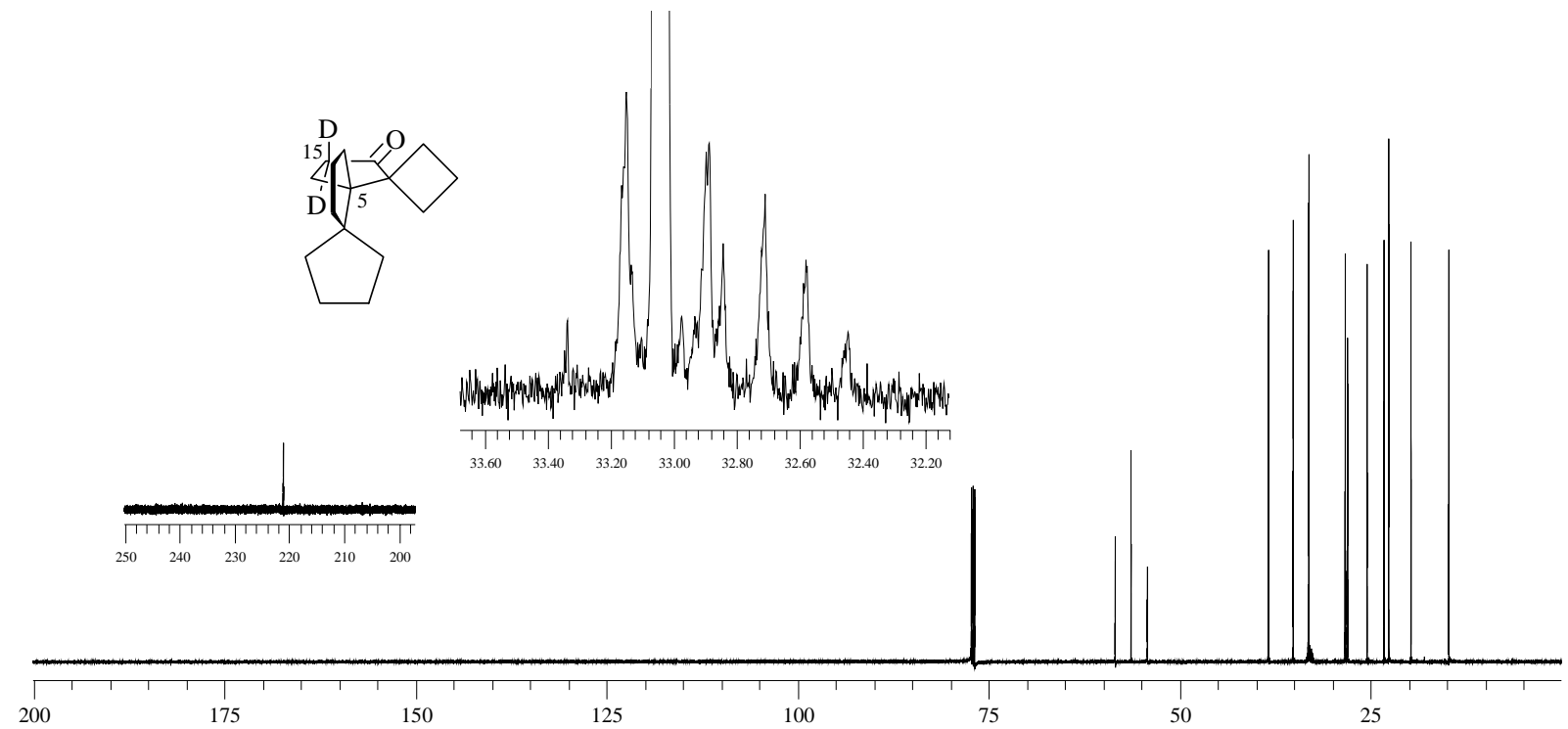

Abb. $110 \quad{ }^{13}$ C-NMR-Spektrum von [15,15- $\left.\mathrm{D}_{2}\right]-\left(5 S^{*}\right)$-Trispiro[3.0.0.4.3.3]hexadecan-16on $\left\{\left[15,15-D_{2}\right]-\left(5 S^{*}\right)-57\right\}$

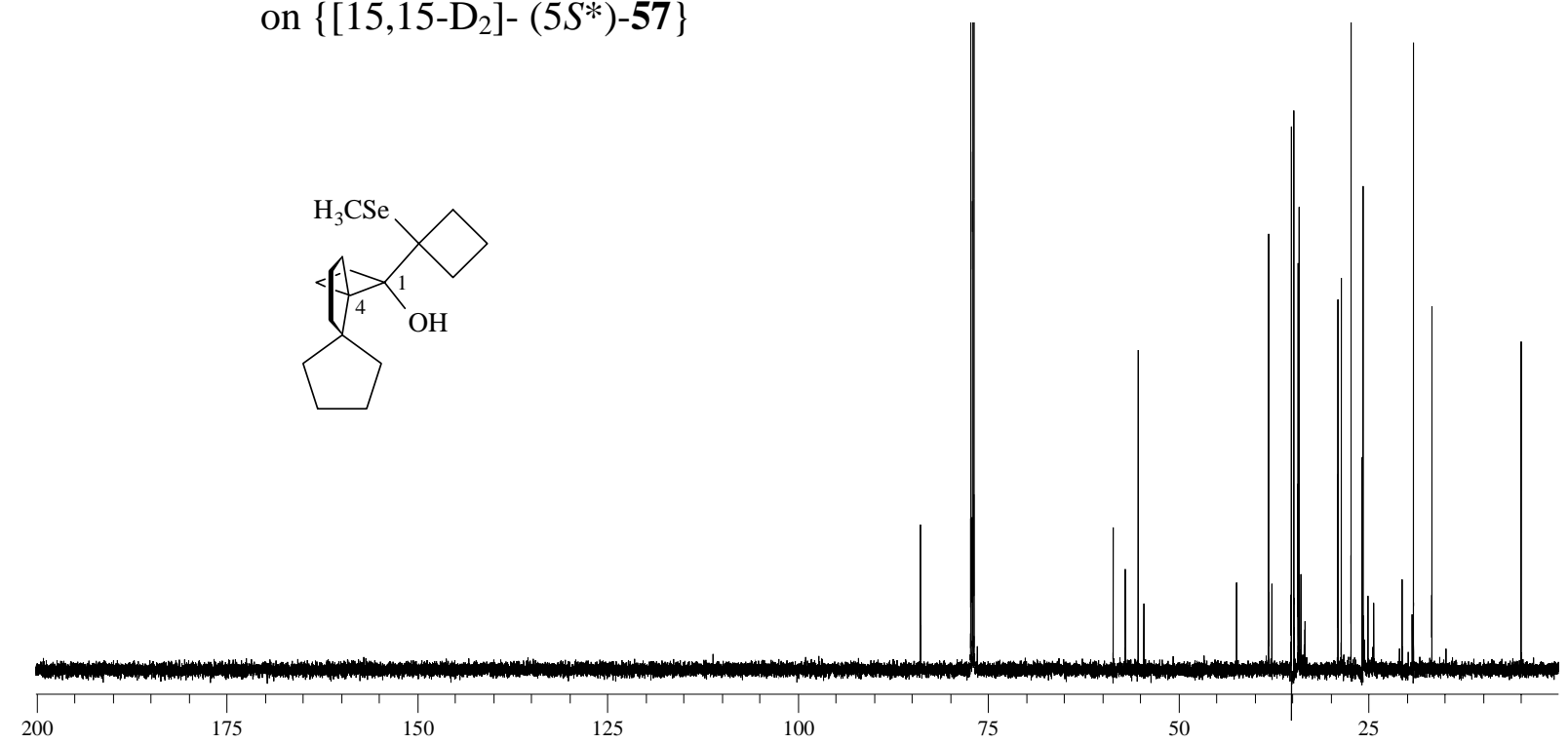

Abb. $111{ }^{13}$ C-NMR-Spektrum von $\left(1 S^{*}, 4 S^{*}\right)-1-\left[\left(1^{\prime}-\right.\right.$ Methylselanyl)cyclobutyl)]dispiro[3.0.4.3]dodecan-1-ol [(1S*,4S*)-58]

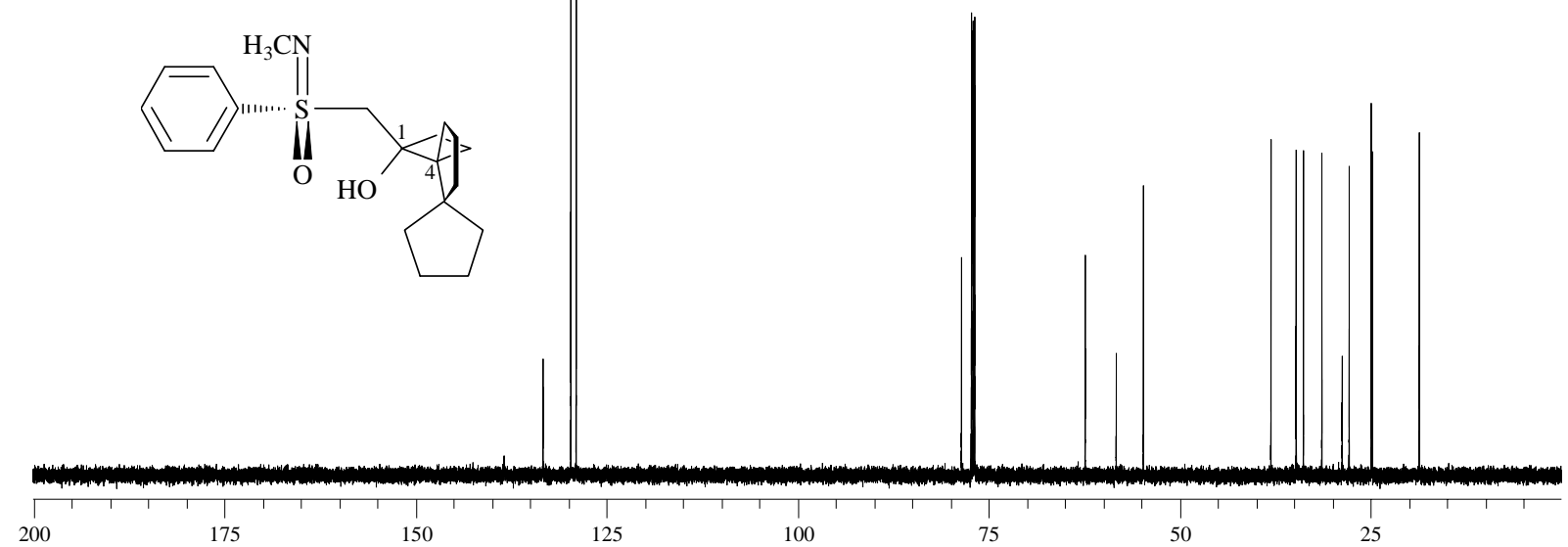

Abb. $112 \quad{ }^{13}$ C-NMR-Spektrum von (SS,1R,4R)-(+)-[(N-Methyl-S-phenylsulfonimidoyl)methyl]-dispiro[3.0.4.3]dodecan-1-ol [(SS,1R,4R)-(+)-59] 

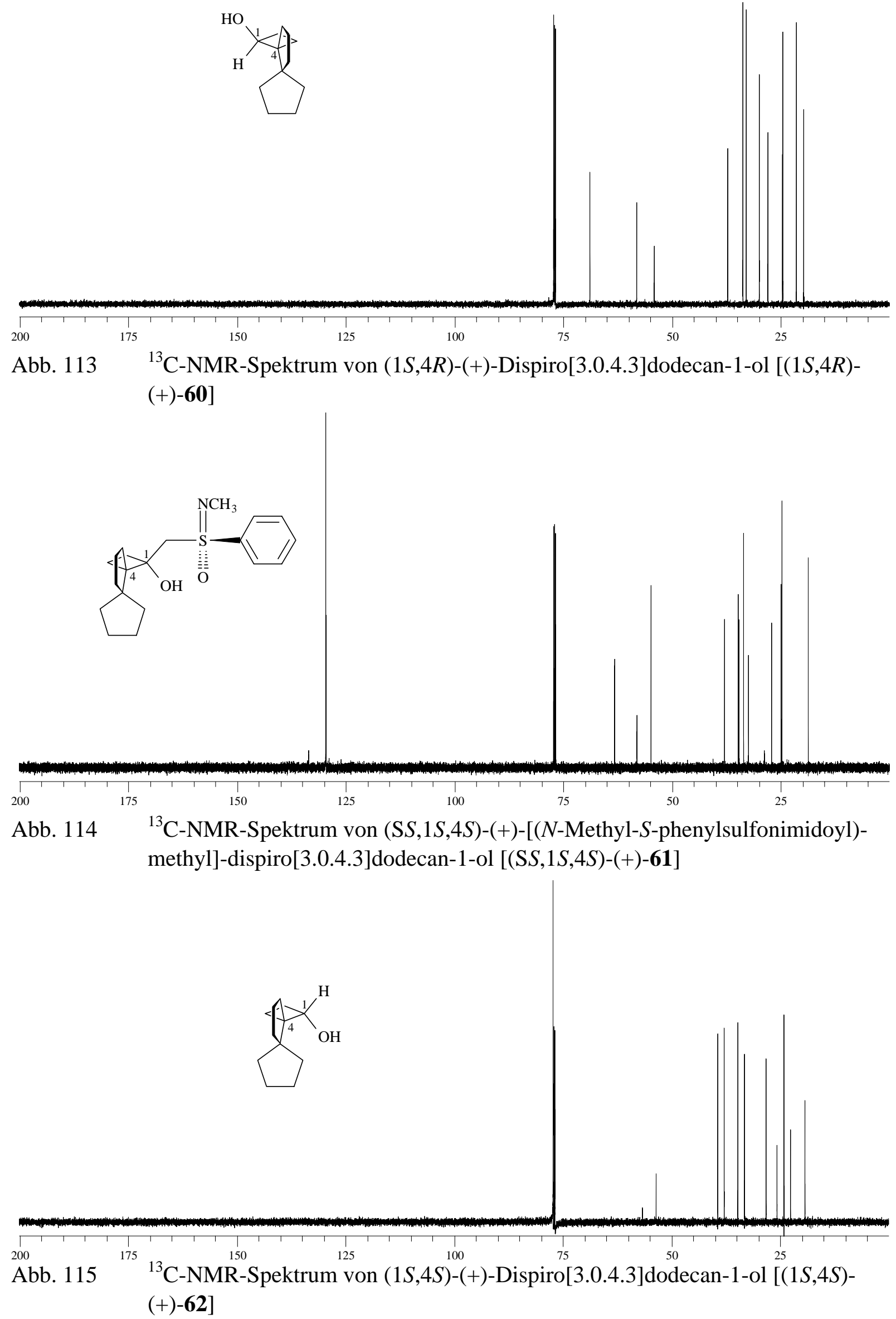

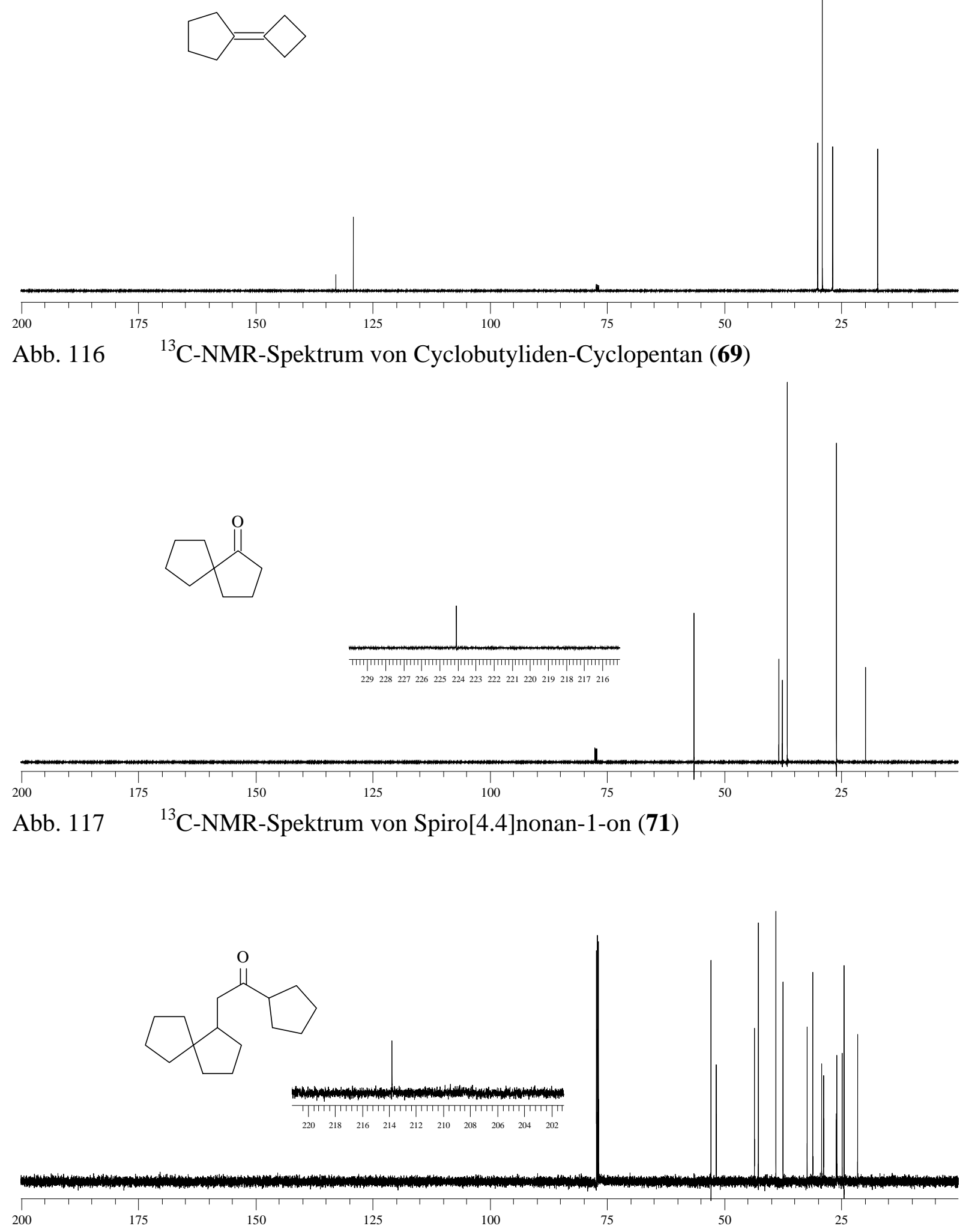

Abb. $118 \quad{ }^{13}$ C-NMR-Spektrum von 1-Cyclopentyl-2-spiro[4.4]non-1-yl-ethanon (75) 

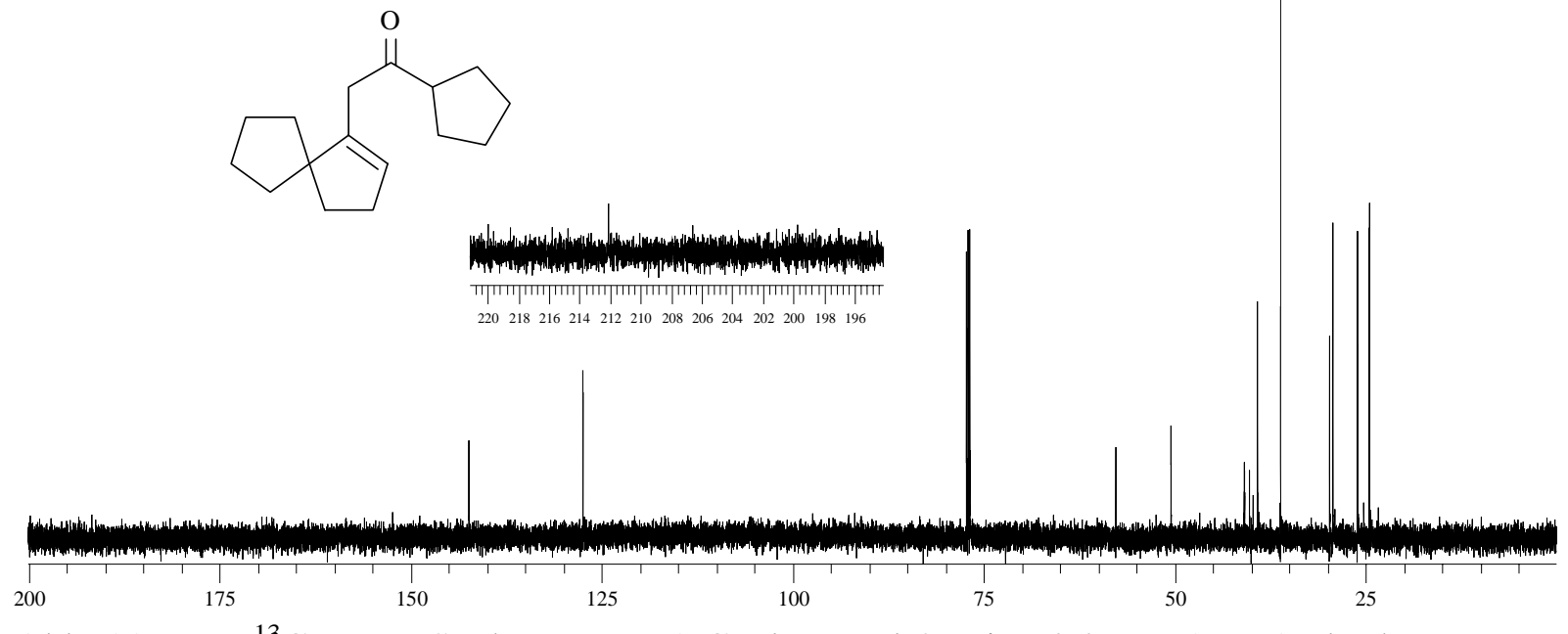

Abb. $119 \quad{ }^{13}$ C-NMR-Spektrum von 1-Cyclopentyl-2-spiro[4.4]non-1-en-1-yl-ethanon (76)
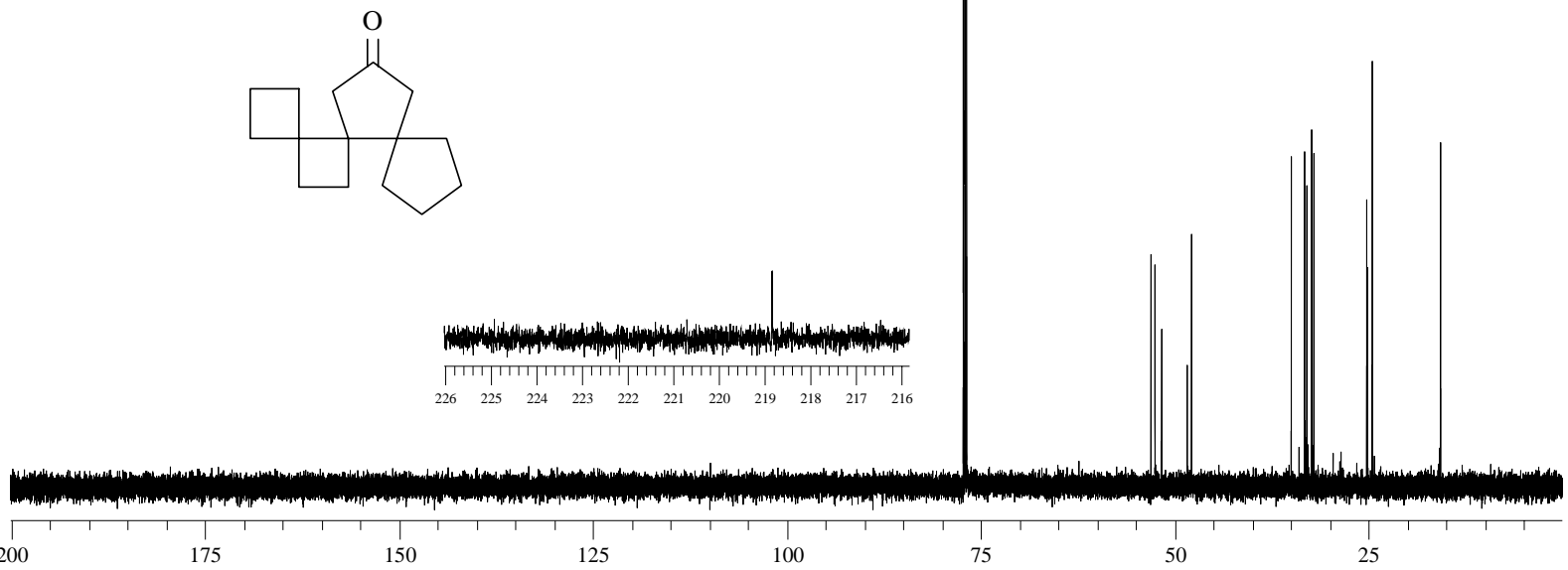

Abb. $120 \quad{ }^{13}$ C-NMR-Spektrum von (5R*)-Trispiro[3.0.0.4.3.2]tetradecan-12-on (77)

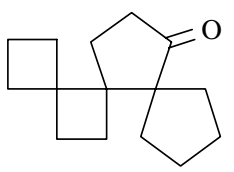

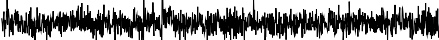

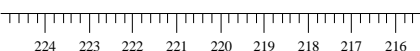

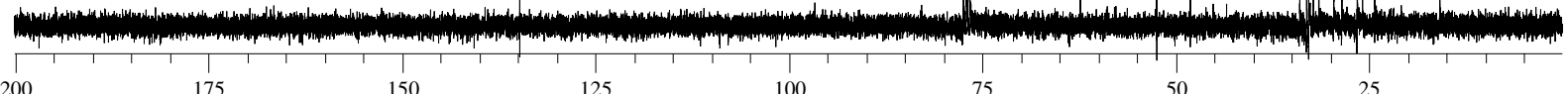

Abb. $121 \quad{ }^{13}$ C-NMR-Spektrum von (5R*)-Trispiro[3.0.0.4.3.2]tetradecan-11-on (78) 

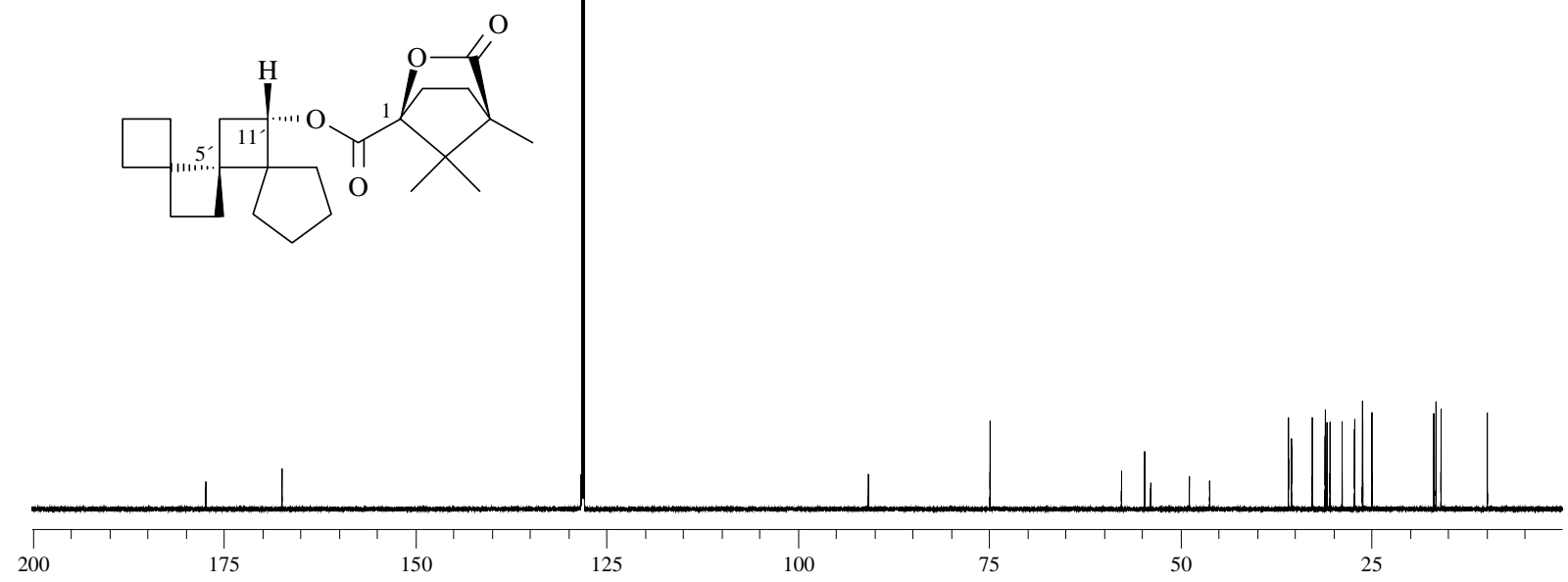

Abb. $122 \quad{ }^{13}$ C-NMR-Spektrum von (1S)-(+)-4,7,7-Trimethyl-3-oxo-2-oxa-bicyclo[2.2.1]heptan-1-carbonsäure( $\left.5^{\prime} S, 11^{\prime} S\right)$-trispiro[3.0.0.4.2.2]tetradecan-11-yl-ester $\left[\left(1 S, 5^{\prime} S, 11^{\prime} S\right)-(+)-79\right]$
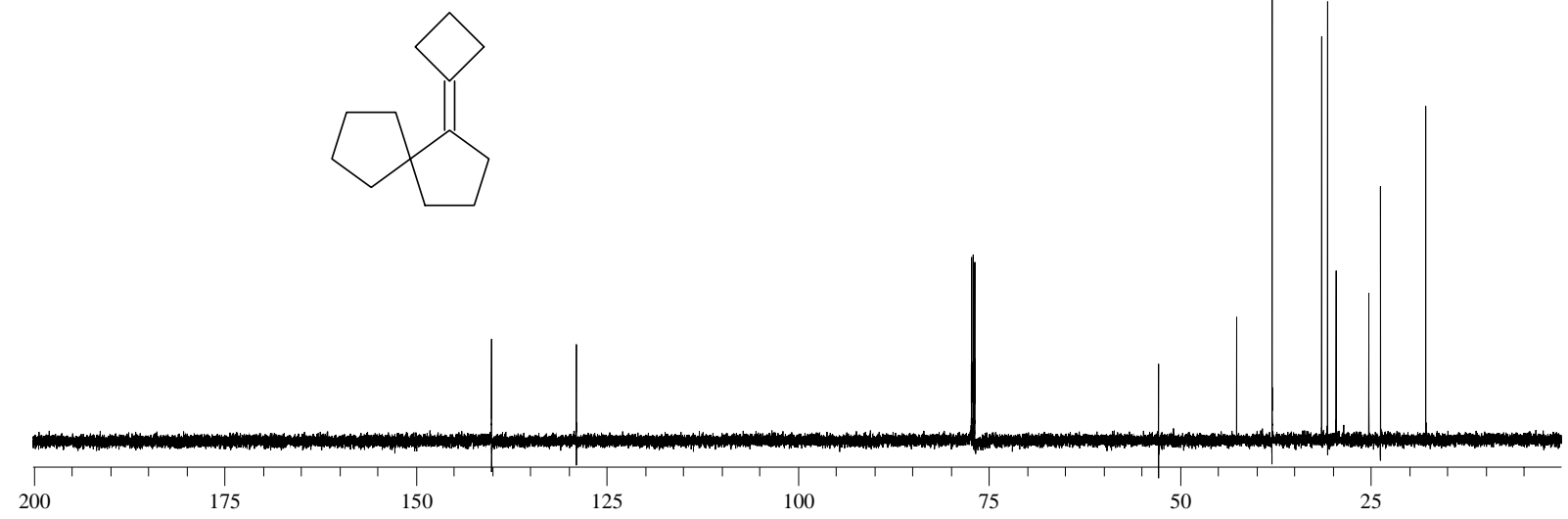

Abb. $123 \quad{ }^{13}$ C-NMR-Spektrum von Cyclobutyliden-spiro[4.4]nonan (85)

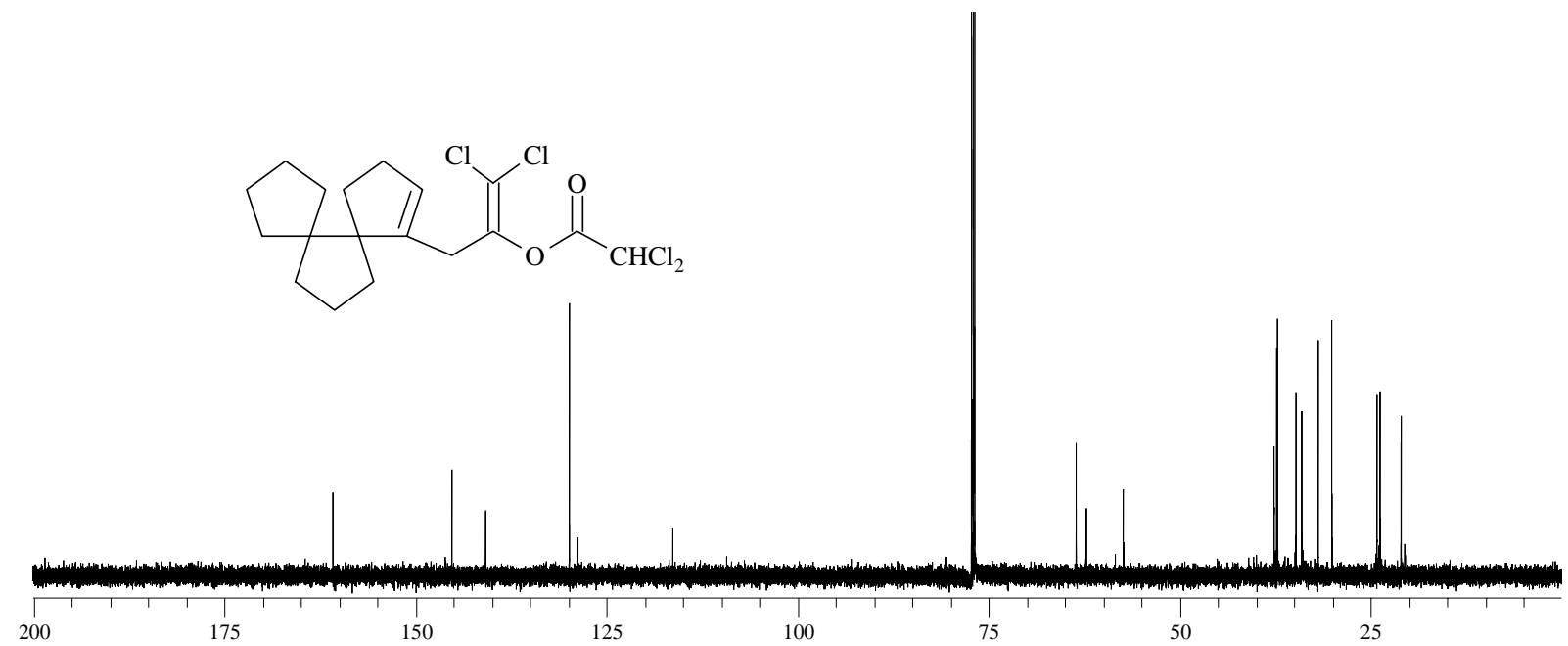

Abb. $124 \quad{ }^{13}$ C-NMR-Spektrum von Dichloressigsäure-2,2-dichlor-1-dispiro[4.0.4.3]tridec-1-en-1-yl-methyl-vinyl-ester (94) 


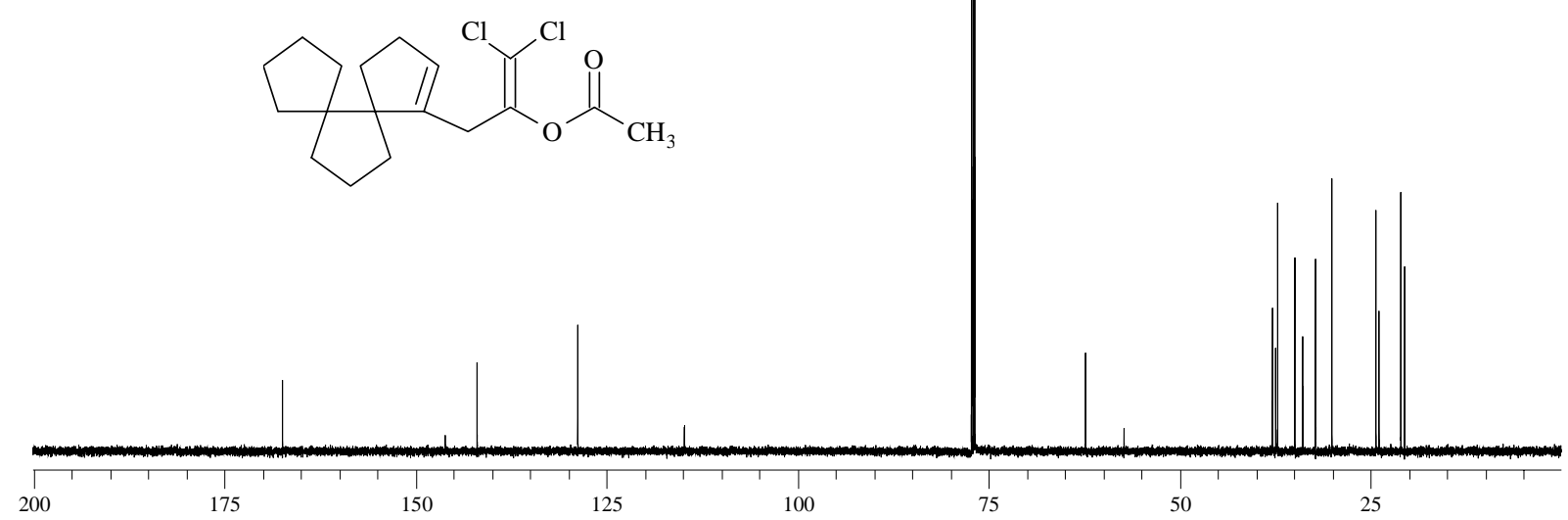

Abb. $125 \quad{ }^{13}$ C-NMR-Spektrum von Essigsäure-2,2-dichlor-1-dispiro[4.0.4.3]tridec-1-en1-yl-methyl-vinyl-ester (95)

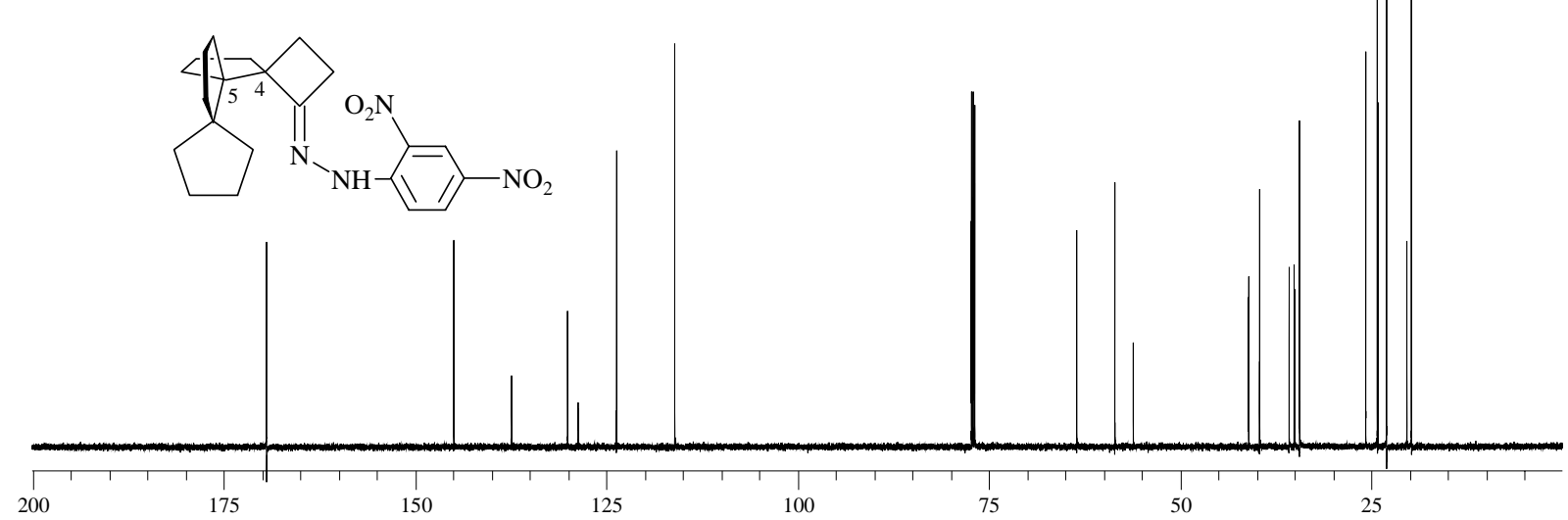

Abb. $126 \quad{ }^{13} \mathrm{C}-\mathrm{NMR}-\mathrm{Spektrum}$ von $\left(4 R^{*}, 5 S^{*}\right)$-N-Trispiro[3.0.0.4.3.3]hexadec-1-yliden$\mathrm{N}^{\prime}$-2,4-dinitrophenyl-hydrazin $\left[\left(4 R^{*}, 5 S^{*}\right)-\mathbf{9 8}\right]$

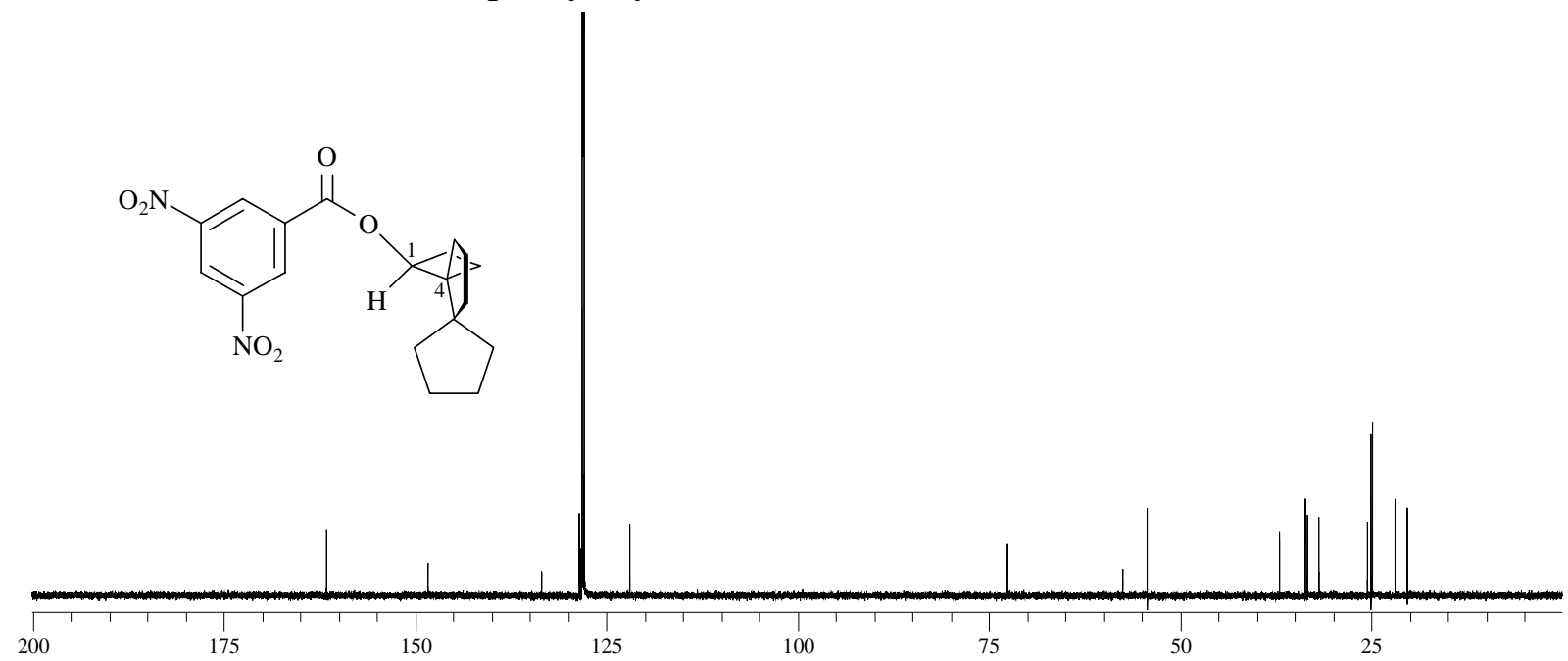

Abb. $127 \quad{ }^{13}$ C-NMR-Spektrum von (1S,4R)-(+)-3,5-Dinitrobenzoesäure-dispiro[3.0.4.3]dodec-1-yl-ester [(1S,4R)-(+)-104] 


\section{ABBILDUNGSVERZEICHNIS}

Abb. 1: $\quad$ Kristallstruktur von (1S)-(+)-4,7,7-Trimethyl-3-oxo-2-oxa-bicyclo[2.2.1]heptan-1-carbonsäure(5'S,11'S)-trispiro[3.0.0.4.2.2]tetradecan-11-yl-ester $\left[\left(1 S, 5^{\prime} S, 11^{\prime} S\right)-(+)-79\right]$

Abb. 2: $\quad$ Kristallstruktur von $\left(4 R^{*}, 5 S^{*}\right)$-N-Trispiro[3.0.0.4.3.3]hexadec-1-yliden-N'-2,4dinitrophenyl-hydrazin [(4R*,5S*)-98]

Abb. 3: $\quad$ Kristallstruktur von $\left(6 S^{*}, 7 S^{*}, 8 R^{*}\right)$-8-Brom-trispiro[4.0.0.4.3.3] on $\left[\left(6 S^{*}, 7 S^{*}, 8 R^{*}\right)-54\right]$

Abb. 4: $\quad$ Kristallstruktur von (SS,1R,4R)-(+)-[(N-Methyl-S-phenylsulfonimidoyl)methyl]-dispiro[3.0.4.3]dodecan-1-ol [(SS,1R,4R)-(+)-59]

Abb. 5: $\quad$ Kristallstruktur von (SS,1S,4S)-(+)-[(N-Methyl-S-phenylsulfonimidoyl)methyl]-dispiro[3.0.4.3]dodecan-1-ol [(SS,1S,4S)-(+)-61]

Abb. 6: $\quad$ Kristallstruktur von (1S,4R)-(+)-3,5-Dinitrobenzoesäure-dispiro[3.0.4.3]dodec1-yl-ester [(1S,4R)-(+)-104]

Abb. 7: $\quad$ Bildungsenthalpien (in kcal/mol) aller Konformationen von (P)-7, (5R)-13, (5S)-14, (5R)-15 und $(P)-\mathbf{8}$ bis 3 kcal über dem absoluten Minimum.

Abb. 8: $\quad$ IR-Spektrum von Trispiro[4.0.0.4.3.3]heptadecan (8)

Abb. 9: $\quad$ IR-Spektrum von (5R*)-Trispiro[3.0.0.4.2.2] tetradecan (13)

Abb. 10: $\quad$ IR-Spektrum von (5R*)-Trispiro[3.0.0.4.3.2] pentadecan (14)

Abb. 11: $\quad$ IR-Spektrum von (5S*)-Trispiro[3.0.0.4.3.3] hexadecan [(5S*)-15]

Abb. 12: $\quad$ IR-Spektrum von 1-(Cyclopentylcarbonyl)-piperidin (23)

Abb. 13: $\quad$ IR-Spektrum von (5R*)-Trispiro[3.0.0.4.2.2]tetradecan-11-on (24)

Abb. 14: $\quad$ IR-Spektrum von $(5 S, 11 S)-(+)$-Trispiro[3.0.0.4.2.2]tetradecan-11-ol [(5S,11S)(+)-28)]

Abb. 15: $\quad$ IR-Spektrum von $(5 R, 11 S)-(+)-T r i s p i r o[3.0 .0 .4 .2 .2]$ tetradecan-11-ol [(5R,11S)-(+)-29)]

Abb. 16: $\quad$ IR-Spektrum von Dispiro[3.1.4.3]tridecan-5-on (31)

Abb. 17: $\quad$ IR-Spektrum von 1-Methylen-dispiro[4.0.4.3]tridecan (33)

Abb. 18: $\quad$ IR-Spektrum von $\left(5 S^{*}, 6 S^{*}\right)$-Trispiro[4.0.0.4.3.3]heptadecan-2-on [(5S*,6 $\left.S^{*}\right)$ 35]

Abb. 19: $\quad$ IR-Spektrum von $\left(1 S^{*}, 5 S^{*}\right)$-1-(1-Ethoxy-vinyl)-dispiro[4.0.4.3]tridecan-1-ol [(1S*,5S*)-41]

Abb. 20: IR-Spektrum von (1S*,5S*)-1-(1-Ethoxy-cyclopropyl)-dispiro[4.0.4.3]tridecan1-ol [(1S*,5S*)-42]

Abb. 21: IR-Spektrum von $\left(4 R^{*}, 5 S^{*}\right)$-Trispiro[3.0.0.4.3.3] $\quad$ hexadecan-1-on [( $\left.4 R^{*}, 5 S^{*}\right)$ 43] 
Abb. 22: $\quad$ IR-Spektrum von $\left(4 R^{*}, 5 S^{*}\right)-1-M e t h y l e n-t r i s p i r o[3.0 .0 .4 .3 .3]$ hexadecan $\left[\left(4 R^{*}, 5 S^{*}\right)-44\right]$

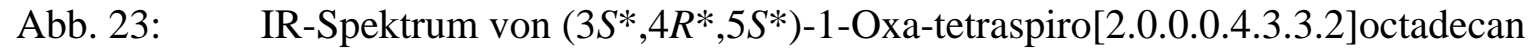
$\left[\left(3 S^{*}, 4 R^{*}, 5 S^{*}\right)-46\right]$

Abb. 24: $\quad$ IR-Spektrum von Cyclopropyliden-spiro[4.4]nonan (49)

Abb. 25: $\quad$ IR-Spektrum von (4S*)-Dispiro[3.0.4.3]dodecan-1-on [(4S*)-51]

Abb. 26: $\quad$ IR-Spektrum von $\left(1 R^{*}, 4 S^{*}\right)$-1-Cyclopent-1-enyl-dispiro[3.0.4.3]dodecan-1-ol $\left[\left(1 R^{*}, 4 S^{*}\right)-52\right]$

Abb. 27: $\quad$ IR-Spektrum von $\left(1 R^{*}, 4 S^{*}\right)$-1-Cyclobut-1-enyl-dispiro[3.0.4.3]dodecan-1-ol $\left[\left(1 R^{*}, 4 S^{*}\right)-53\right]$

Abb. 28: $\quad$ IR-Spektrum von $\left(6 S^{*}, 7 S^{*}, 8 R^{*}\right)$-8-Brom-trispiro[4.0.0.4.3.3]heptadecan-12-on $\left[\left(6 S^{*}, 7 S^{*}, 8 R^{*}\right)-54\right]$

Abb. 29: $\quad$ IR-Spektrum von (6S*)-Trispiro[4.0.0.4.3.3] heptadecan-12-on [(6S*)-55]

Abb. 30: IR-Spektrum von $\left(1 R^{*}, 4 S^{*}, 5 S^{*}\right)$-1-Brom-trispiro[3.0.0.4.3.3]hexadecan-16-on $\left[\left(1 R^{*}, 4 S^{*}, 5 S^{*}\right)-56\right]$

Abb. 31: IR-Spektrum von (5S*)-Trispiro[3.0.0.4.3.3] $\quad$ Ihexadecan-16-on [(5S*)-57]

Abb. 32: $\quad$ IR-Spektrum von (SS,1R,4R)-(+)-[( $N$-Methyl-S-phenylsulfonimidoyl)methyl]dispiro[3.0.4.3]dodecan-1-ol [(SS,1R,4R)-(+)-59]

Abb. 33: $\quad$ IR-Spektrum von (1S,4R)-(+)-Dispiro[3.0.4.3]dodecan-1-ol [(1S,4R)-(+)-60]

Abb. 34: $\quad$ IR-Spektrum von (SS,1S,4S)-(+)-[(N-Methyl-S-phenylsulfonimidoyl)methyl]dispiro[3.0.4.3]dodecan-1-ol [(SS,1S,4S)-(+)-61]

Abb. 35: $\quad$ IR-Spektrum von (1S,4S)-(+)-Dispiro[3.0.4.3]dodecan-1-ol [(1S,4S)-(+)-62]

Abb. 36: IR-Spektrum von 1-Cyclopentyl-2-spiro[4.4]non-1-yl-ethanon (75)

Abb. 37: $\quad$ IR-Spektrum von 1-Cyclopentyl-2-spiro[4.4]non-1-en-1-yl-ethanon (76)

Abb. 38: $\quad$ IR-Spektrum von (5R*)-Trispiro[3.0.0.4.3.2]tetradecan-12-on (77)

Abb. 39: $\quad$ IR-Spektrum von (5R*)-Trispiro[3.0.0.4.3.2] tetradecan-11-on (78)

Abb. 40: $\quad$ IR-Spektrum von (1S)-(+)-4,7,7-Trimethyl-3-oxo-2-oxa-bicyclo[2.2.1]heptan1-carbonsäure(5'S,11'S)-trispiro[3.0.0.4.2.2]tetradecan-11-yl-ester $\left[\left(1 S, 5^{\prime} S, 11^{\prime} S\right)-(+)-79\right]$

Abb. 41: $\quad$ IR-Spektrum von Cyclobutyliden-spiro[4.4]nonan (85)

Abb. 42: $\quad$ IR-Spektrum von Essigsäure-2,2-dichlor-1-dispiro[4.0.4.3]tridec-1-en-1-ylmethyl-vinyl-ester (95)

Abb. 43: IR-Spektrum von $\left(4 R^{*}, 5 S^{*}\right)$-N-Trispiro[3.0.0.4.3.3] $\quad$ hexadec-1-yliden- $\mathrm{N}^{\prime}-2,4-$ dinitrophenyl-hydrazin [(4R*,5S*)-98]

Abb. 44: $\quad$ IR-Spektrum von (1S,4R)-(+)-3,5-Dinitrobenzoesäure-dispiro[3.0.4.3]dodec-1yl-ester $[(1 S, 4 R)-(+)-104]$

Abb. 45: $\quad{ }^{1} \mathrm{H}-\mathrm{NMR}-\mathrm{Spektrum}$ von Trispiro[4.0.0.4.3.3] heptadecan (8)

Abb. 46: $\quad{ }^{1}$ H-NMR-Spektrum von (5R*)-Trispiro[3.0.0.4.2.2] tetradecan (13)

Abb. 47: $\quad{ }^{1} \mathrm{H}-\mathrm{NMR}-\mathrm{Spektrum}$ von (5R*)-Trispiro[3.0.0.4.3.2]pentadecan (14) 
Abb. 48: $\quad{ }^{1}$ H-NMR-Spektrum von (5S*)-Trispiro[3.0.0.4.3.3]hexadecan [(5S*)-15]

Abb 49: $\quad{ }^{1}$ H-NMR-Spektrum von 1-(Cyclopentylcarbonyl)-piperidin (23)

Abb. 50: $\quad{ }^{1}$ H-NMR-Spektrum von (5R*)-Trispiro[3.0.0.4.2.2]tetradecan-11-on (24)

Abb. 51: $\quad{ }^{1}$ H-NMR-Spektrum von (5S,11S)-(+)-Trispiro[3.0.0.4.2.2]tetradecan-11-ol [(5S,11S)-(+)-28)]

Abb. 52: $\quad{ }^{1} \mathrm{H}-\mathrm{NMR}-\mathrm{Spektrum}$ von (5R,11S)-(+)-Trispiro[3.0.0.4.2.2]tetradecan-11-ol [(5R,11S)-(+)-29)]

Abb. 53: $\quad{ }^{1}$ H-NMR-Spektrum von Dispiro[3.1.4.3]tridecan-5-on (31)

Abb. 54: $\quad{ }^{1}$ H-NMR-Spektrum von 1-Methylen-dispiro[4.0.4.3]tridecan (33)

Abb. 55: $\quad{ }^{1} \mathrm{H}-\mathrm{NMR}-\mathrm{Spektrum}$ von (5S*,6S*)-Trispiro[4.0.0.4.3.3] [(5S*,6S*)-35]

Abb. 56: $\quad{ }^{1} \mathrm{H}-\mathrm{NMR}-\mathrm{Spektrum}$ von $\left(1 S^{*}, 5 S^{*}\right)-1-(1-E t h o x y-v i n y l)-d i s p i r o[4.0 .4 .3]$ tridecan1-ol [(1S*,5S*)-41]

Abb. 57: $\quad{ }^{1}$ H-NMR-Spektrum von (1S*,5S*)-1-(1-Ethoxy-cyclopropyl)-dispiro[4.0.4.3]tridecan-1-ol [(1S*,5S*)-42]

Abb. 58: $\quad{ }^{1} \mathrm{H}-\mathrm{NMR}-\mathrm{Spektrum}$ von $\left(4 R^{*}, 5 S^{*}\right)$-Trispiro[3.0.0.4.3.3] hexadecan-1-on [(4R*,5S*)-43]

Abb. 59: $\quad{ }^{1} \mathrm{H}-\mathrm{NMR}-\mathrm{Spektrum}$ von $\left(4 R^{*}, 5 S^{*}\right)$-1-Methylen-trispiro[3.0.0.4.3.3] hexadecan $\left[\left(4 R^{*}, 5 S^{*}\right)-44\right]$

Abb. 60: $\quad{ }^{1} \mathrm{H}-\mathrm{NMR}-\mathrm{Spektrum}$ von $\left(3 S^{*}, 4 R^{*}, 5 S^{*}\right)-1-O x a-t e t r a s p i r o[2.0 .0 .0 .4 .3 .3 .2]$ octadecan $\left[\left(3 S^{*}, 4 R^{*}, 5 S^{*}\right)-46\right]$

Abb. 61: $\quad{ }^{1}$ H-NMR-Spektrum von Cyclopropyliden-spiro[4.4]nonan (49)

Abb. 62: $\quad{ }^{1}$ H-NMR-Spektrum von (4S*)-Dispiro[3.0.4.3]dodecan-1-on [(4S*)-51]

Abb. 63: $\quad{ }^{1} \mathrm{H}-\mathrm{NMR}-\mathrm{Spektrum}$ von $\left(1 R^{*}, 4 S^{*}\right)$-1-Cyclopent-1-enyl-dispiro[3.0.4.3]dodecan-1-ol [(1R*,4S*)-52]

Abb. 64: $\quad{ }^{1} \mathrm{H}-\mathrm{NMR}-\mathrm{Spektrum}$ von $\left(1 R^{*}, 4 S^{*}\right)$-1-Cyclobut-1-enyl-dispiro[3.0.4.3]dodecan1-ol $\left[\left(1 R^{*}, 4 S^{*}\right)-53\right]$

Abb. 65: $\quad{ }^{1} \mathrm{H}-\mathrm{NMR}-\mathrm{Spektrum}$ von $\left(6 S^{*}, 7 S^{*}, 8 R^{*}\right)$-8-Brom-trispiro[4.0.0.4.3.3]heptadecan-12-on [(6S*,7S*,8R*)-54]

Abb. 66: $\quad{ }^{1} \mathrm{H}-\mathrm{NMR}-\mathrm{Spektrum}$ von (6S*)-Trispiro[4.0.0.4.3.3]heptadecan-12-on [(6S*)55]

Abb. 67: $\quad{ }^{1} \mathrm{H}-\mathrm{NMR}-\mathrm{Spektrum}$ von $\left(1 R^{*}, 4 S^{*}, 5 S^{*}\right)-1-B r o m-t r i s p i r o[3.0 .0 .4 .3 .3]$ hexadecan16-on $\left[\left(1 R^{*}, 4 S^{*}, 5 S^{*}\right)-56\right]$

Abb. 68: $\quad{ }^{1}$ H-NMR-Spektrum von (5S*)-Trispiro[3.0.0.4.3.3] hexadecan-16-on [(5S*)-57]

Abb. 69: $\quad{ }^{1}$ H-NMR-Spektrum von $\left(1 S^{*}, 4 S^{*}\right)-1-\left[\left(1^{\prime}\right.\right.$-Methylselanyl)cyclobutyl)]dispiro[3.0.4.3]dodecan-1-ol [(1S*,4S*)-58]

Abb. 70: $\quad{ }^{1} \mathrm{H}-\mathrm{NMR}-\mathrm{Spektrum}$ von (SS,1R,4R)-(+)-[(N-Methyl-S-phenylsulfonimidoyl)methyl]-dispiro[3.0.4.3]dodecan-1-ol [(SS,1R,4R)-(+)-59] 
Abb. 71: $\quad{ }^{1}$ H-NMR-Spektrum von (1S,4R)-(+)-Dispiro[3.0.4.3]dodecan-1-ol [(1S,4R)(+)-60]

Abb. 72: $\quad{ }^{1}$ H-NMR-Spektrum von (SS,1S,4S)-(+)-[(N-Methyl-S-phenylsulfonimidoyl)methyl]-dispiro[3.0.4.3]dodecan-1-ol [(SS,1S,4S)-(+)-61]

Abb. 73: $\quad{ }^{1}$ H-NMR-Spektrum von $(1 S, 4 S)-(+)-D i s p i r o[3.0 .4 .3]$ dodecan-1-ol [(1S,4S)-(+)62]

Abb. 74: $\quad{ }^{1} \mathrm{H}-\mathrm{NMR}-\mathrm{Spektrum}$ von Cyclobutyliden-Cyclopentan (69)

Abb. 75: $\quad{ }^{1}$ H-NMR-Spektrum von Spiro[4.4]nonan-1-on (71)

Abb. 76: $\quad{ }^{1}$ H-NMR-Spektrum von 1-Cyclopentyl-2-spiro[4.4]non-1-yl-ethanon (75)

Abb. 77: $\quad{ }^{1}$ H-NMR-Spektrum von 1-Cyclopentyl-2-spiro[4.4]non-1-en-1-yl-ethanon (76)

Abb. 78: $\quad{ }^{1} \mathrm{H}-\mathrm{NMR}-\mathrm{Spektrum}$ von (5R*)-Trispiro[3.0.0.4.3.2] tetradecan-12-on (77)

Abb. 79: $\quad{ }^{1} \mathrm{H}-\mathrm{NMR}-\mathrm{Spektrum}$ von (5R*)-Trispiro[3.0.0.4.3.2] tetradecan-11-on (78)

Abb. 80: $\quad{ }^{1}$ H-NMR-Spektrum von (1S)-(+)-4,7,7-Trimethyl-3-oxo-2-oxa-bicyclo[2.2.1]heptan-1-carbonsäure(5'S,11'S)-trispiro[3.0.0.4.2.2]tetradecan-11-yl-ester [(1S,5'S,11'S)-(+)-79]

Abb. 81: $\quad{ }^{1} \mathrm{H}-\mathrm{NMR}-\mathrm{Spektrum}$ von Cyclobutyliden-spiro[4.4]nonan (85)

Abb. 82: $\quad{ }^{1}$ H-NMR-Spektrum von Dichloressigsäure-2,2-dichlor-1-dispiro[4.0.4.3]tridec1-en-1-yl-methyl-vinyl-ester (94)

Abb. 83: $\quad{ }^{1}$ H-NMR-Spektrum von Essigsäure-2,2-dichlor-1-dispiro[4.0.4.3]tridec-1-en-1yl-methyl-vinyl-ester (95)

Abb. 84: $\quad{ }^{1} \mathrm{H}-\mathrm{NMR}-\mathrm{Spektrum}$ von $\left(4 R^{*}, 5 S^{*}\right)-\mathrm{N}-\mathrm{Trispiro}[3.0 .0 .4 .3 .3]$ hexadec-1-yliden$\mathrm{N}^{\prime}$-2,4-dinitrophenyl-hydrazin [(4R*,5S*)-98]

Abb. 85: $\quad{ }^{1} \mathrm{H}-\mathrm{NMR}-\mathrm{Spektrum}$ von $(1 S, 4 R)-(+)-3,5-$ Dinitrobenzoesäure-dispiro[3.0.4.3]dodec-1-yl-ester [(1S,4R)-(+)-104]

Abb. 86: $\quad{ }^{13}$ C-NMR-Spektrum von Trispiro[4.0.0.4.3.3] heptadecan (8)

Abb. 87: $\quad{ }^{13} \mathrm{C}-\mathrm{NMR}-\mathrm{Spektrum}$ von (5R*)-Trispiro[3.0.0.4.2.2] tetradecan (13)

Abb. 88: $\quad{ }^{13}$ C-NMR-Spektrum von (5R*)-Trispiro[3.0.0.4.3.2]pentadecan (14)

Abb. 89: $\quad{ }^{13}$ C-NMR-Spektrum von (5S*)-Trispiro[3.0.0.4.3.3] hexadecan [(5S*)-15]

Abb. 90: $\quad{ }^{13}$ C-NMR-Spektrum von 1-(Cyclopentylcarbonyl)-piperidin (23)

Abb. 91: $\quad{ }^{13}$ C-NMR-Spektrum von (5R*)-Trispiro[3.0.0.4.2.2]tetradecan-11-on (24)

Abb. 92: $\quad{ }^{13}$ C-NMR-Spektrum von $(5 S, 11 S)-(+)-T r i s p i r o[3.0 .0 .4 .2 .2]$ tetradecan-11-ol [(5S,11S)-(+)-28)]

Abb. 93: $\quad{ }^{13}$ C-NMR-Spektrum von $(5 R, 11 S)-(+)-T r i s p i r o[3.0 .0 .4 .2 .2]$ tetradecan-11-ol [(5R,11S)-(+)-29)]

Abb. 94: $\quad{ }^{13}$ C-NMR-Spektrum von Dispiro[3.1.4.3]tridecan-5-on (31)

Abb. 95: $\quad{ }^{13} \mathrm{C}-\mathrm{NMR}-S p e k t r u m$ von 1-Methylen-dispiro[4.0.4.3]tridecan (33)

Abb. 96: $\quad{ }^{13} \mathrm{C}-\mathrm{NMR}-\mathrm{Spektrum}$ von $\left(5 S^{*}, 6 S^{*}\right)$-Trispiro[4.0.0.4.3.3] heptadecan-2-on $\left[\left(5 S^{*}, 6 S^{*}\right)-35\right]$ 
Abb. 97: $\quad{ }^{13}$ C-NMR-Spektrum von (1S*,5S*)-1-(1-Ethoxy-vinyl)-dispiro[4.0.4.3]tridecan-1-ol [(1S*,5S*)-41]

Abb. 98: $\quad{ }^{13}$ C-NMR-Spektrum von (1S*,5S*)-1-(1-Ethoxy-cyclopropyl)-dispiro[4.0.4.3]tridecan-1-ol [(1S*,5S*)-42]

Abb. 99: $\quad{ }^{13}$ C-NMR-Spektrum von $\left(4 R^{*}, 5 S^{*}\right)$-Trispiro[3.0.0.4.3.3] hexadecan-1-on [(4R*,5S*)-43]

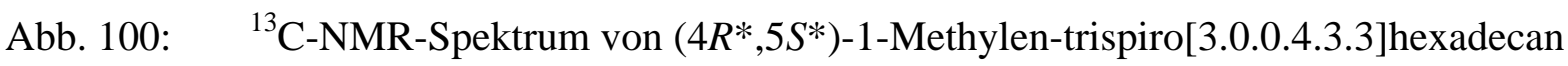
$\left[\left(4 R^{*}, 5 S^{*}\right)-44\right]$

Abb. 101: $\quad{ }^{13}$ C-NMR-Spektrum von $\left(3 S^{*}, 4 R^{*}, 5 S^{*}\right)$-1-Oxa-tetraspiro[2.0.0.0.4.3.3.2]octadecan $\left[\left(3 S^{*}, 4 R^{*}, 5 S^{*}\right)-46\right]$

Abb. 102: $\quad{ }^{13}$ C-NMR-Spektrum von Cyclopropyliden-spiro[4.4]nonan (49)

Abb. 103: $\quad{ }^{13}$ C-NMR-Spektrum von (4S*)-Dispiro[3.0.4.3]dodecan-1-on [(4S*)-51]

Abb. 104: $\quad{ }^{13}$ C-NMR-Spektrum von $\left(1 R^{*}, 4 S^{*}\right)$-1-Cyclopent-1-enyl-dispiro[3.0.4.3]dodecan-1-ol [(1R*,4S*)-52]

Abb. 105: $\quad{ }^{13}$ C-NMR-Spektrum von $\left(1 R^{*}, 4 S^{*}\right)$-1-Cyclobut-1-enyl-dispiro[3.0.4.3]dodecan-1-ol [(1R*,4S*)-53]

Abb. 106: $\quad{ }^{13} \mathrm{C}-\mathrm{NMR}-\mathrm{Spektrum}$ von $\left(6 S^{*}, 7 S^{*}, 8 R^{*}\right)$-8-Brom-trispiro[4.0.0.4.3.3]heptadecan-12-on [(6S*,7S*,8R*)-54]

Abb. 107: $\quad{ }^{13}$ C-NMR-Spektrum von (6S*)-Trispiro[4.0.0.4.3.3] heptadecan-12-on [(6S*)55]

Abb. 108: $\quad{ }^{13} \mathrm{C}-\mathrm{NMR}-\mathrm{Spektrum}$ von $\left(1 R^{*}, 4 S^{*}, 5 S^{*}\right)-1$-Brom-trispiro[3.0.0.4.3.3] hexadecan-16-on [(1R*,4S*,5S*)-56]

Abb. 109: $\quad{ }^{13}$ C-NMR-Spektrum von (5S*)-Trispiro[3.0.0.4.3.3] hexadecan-16-on [(5S*)57]

Abb. 110: $\quad{ }^{13}$ C-NMR-Spektrum von [15,15- $\left.\mathrm{D}_{2}\right]-\left(5 S^{*}\right)$-Trispiro[3.0.0.4.3.3]hexadecan-16on $\left\{\left[15,15-\mathrm{D}_{2}\right]-\left(5 S^{*}\right)-57\right\}$

Abb. 111: $\quad{ }^{13}$ C-NMR-Spektrum von $\left(1 S^{*}, 4 S^{*}\right)-1-\left[\left(1^{\prime}\right.\right.$-Methylselanyl)cyclobutyl)]dispiro[3.0.4.3]dodecan-1-ol [(1S*,4S*)-58]

Abb. 112: $\quad{ }^{13} \mathrm{C}-\mathrm{NMR}-\mathrm{Spektrum}$ von (SS,1R,4R)-(+)-[(N-Methyl-S-phenylsulfonimidoyl)methyl]-dispiro[3.0.4.3]dodecan-1-ol [(SS,1R,4R)-(+)-59]

Abb. 113: $\quad{ }^{13}$ C-NMR-Spektrum von $(1 S, 4 R)-(+)-D i s p i r o[3.0 .4 .3]$ dodecan-1-ol [(1S,4R)(+)-60]

Abb. 114: $\quad{ }^{13}$ C-NMR-Spektrum von (SS,1S,4S)-(+)-[(N-Methyl-S-phenylsulfonimidoyl)methyl]-dispiro[3.0.4.3]dodecan-1-ol [(SS,1S,4S)-(+)-61]

Abb. 115: $\quad{ }^{13}$ C-NMR-Spektrum von $(1 S, 4 S)-(+)$-Dispiro[3.0.4.3]dodecan-1-ol [(1S,4S)$(+)-62]$

Abb. 116: $\quad{ }^{13}$ C-NMR-Spektrum von Cyclobutyliden-Cyclopentan (69)

Abb. 117: $\quad{ }^{13}$ C-NMR-Spektrum von Spiro[4.4]nonan-1-on (71)

Abb. 118: $\quad{ }^{13}$ C-NMR-Spektrum von 1-Cyclopentyl-2-spiro[4.4]non-1-yl-ethanon (75) 
Abb. 119: $\quad{ }^{13}$ C-NMR-Spektrum von 1-Cyclopentyl-2-spiro[4.4]non-1-en-1-yl-ethanon (76)

Abb. 120: $\quad{ }^{13}$ C-NMR-Spektrum von (5R*)-Trispiro[3.0.0.4.3.2]tetradecan-12-on (77)

Abb. 121: $\quad{ }^{13}$ C-NMR-Spektrum von (5R*)-Trispiro[3.0.0.4.3.2]tetradecan-11-on (78)

Abb. 122: $\quad{ }^{13}$ C-NMR-Spektrum von (1S)-(+)-4,7,7-Trimethyl-3-oxo-2-oxa-bicyclo[2.2.1]heptan-1-carbonsäure(5'S,11'S)-trispiro[3.0.0.4.2.2] tetradecan-11-yl-ester [(1S,5'S,11'S)-(+)-79]

Abb. 123: $\quad{ }^{13}$ C-NMR-Spektrum von Cyclobutyliden-spiro[4.4]nonan (85)

Abb. 124: $\quad{ }^{13}$ C-NMR-Spektrum von Dichloressigsäure-2,2-dichlor-1-dispiro[4.0.4.3]tridec-1-en-1-yl-methyl-vinyl-ester (94)

Abb. 125: $\quad{ }^{13}$ C-NMR-Spektrum von Essigsäure-2,2-dichlor-1-dispiro[4.0.4.3]tridec-1-en1-yl-methyl-vinyl-ester (95)

Abb. 126: $\quad{ }^{13}$ C-NMR-Spektrum von $\left(4 R^{*}, 5 S^{*}\right)-N-T r i s p i r o[3.0 .0 .4 .3 .3]$ hexadec-1-yliden$\mathrm{N}^{\prime}$-2,4-dinitrophenyl-hydrazin [(4R*,5S*)-98]

Abb. 127: $\quad{ }^{13}$ C-NMR-Spektrum von (1S,4R)-(+)-3,5-Dinitrobenzoesäure-dispiro[3.0.4.3]dodec-1-yl-ester [(1S,4R)-(+)-104] 


\section{LITERATURVERZEICHNIS}

(1) de Meijere, A.; Khlebnikov, A. F.; Kostikov, R. R.; Kozhuskov, S. I.; Schreiner, P. R.; Wittkopp, A.; Yufit, D. S. Angew. Chem., Int. Ed. Engl. 1999, 38, 3474-3477.

(2) de Meijere, A.; Khlebnikov, A. F.; Kozhuskov, S. I.; Kostikov, R. R.; Schreiner, P. R.; Rinderspacher, C.; Menzel, H.; Yufit, D. S.; Howard, J. A. K. Chem. Eur. J. 2002, 828-842.

(3) de Meijere, A.; F. Khlebnikov, A. F.; Kozhuskov, S. I.; Miyazawa, K.; Frank, D.; Schreiner, P. R.; Rinderspacher, C.; Yufit, D. S.; Howard, J. A. K. Angew. Chem., Int. Ed. Engl. 2004, 43, 6553-6557.

(4) Fitjer, L.; Gerke, R.; Weiser, J.; Bunkoczi, G.; Debreczeni, J. E. Tetrahedron 2003, 59, 4443-4449.

(5) Fitjer, L.; Kanschik, A.; Gerke, R. Tetrahedron 2004, 60, 1205-1213.

(6) Gange, D.; Magnus, P.; Bass, L.; Arnold, E. V.; Clardy, J. J. Am. Chem. Soc. 1980, 102, 2134-2135.

(7) Trost, B. M.; Shi, Y. J. Am. Chem. Soc. 1991, 113, 701-703.

(8) Trost, B. M.; Shi, Y. J. Am. Chem. Soc. 1993, 115, 9431-9438.

(9) Prof. Dr. Grimme, S., Privatmitteilung.

(10) Gaini-Rahimi, S.; Steeneck, C.; Meyer, I.; Fitjer, L.; Pauer, F.; Noltemeyer, M. Tetrahedron 1999, 55, 3905-3916.

(11) Vergleiche die Spannungsenergien (kcal/mol) von Cyclobutan (26.9) und Cyclopentan (7.2): Schleyer, P. v. R.; William, J. E., Blanchard, K. R. J. Am. Chem. Soc. 1970, 92, 2377-2386.

(12) Für Umlagerungen cyclisch 1,1-verknüpfter Polycyclobutane, siehe: (a) Wehle, D.; Schormann, N.; Fitjer, L., Chem. Ber. 1988, 121, 2171-2177. (b) Quabeck, U.; Fitjer, L. Angew. Chem., Int. Ed. Engl. 1987, 26, 1023-1025.

(13) Sprague, J. T.; Tai, J. C.; Yuh, Y.; Allinger, N. L.; J. Comput. Chem. 1987, 8, 581603.

(14) Zu Reduktionen von Cyclobutanonen mit Bäckerhefe, siehe: (a) Newton, R. F.; Paton, J.; Reynolds, D. P.; Young, S.; Roberts, S. M. J. Chem. Soc. Chem. Commun. 1979, 908-909. (b) Dawson, M. J.; Lawrence, G. C.; Lilley, G.; Todd, M.; Noble, D.; Green, S. M.; Roberts, S. M.; Wallace, T. W.; Newton, R. F.; Carter, M. C.; Hallet, P.; Paton, J.; Reynolds, D. P.; Young, S. J. Chem. Soc., Perkin Trans. 1 1983, 2119-2125. (c) Lowe, G.; Swain, S. J. Chem. Soc., Perkin Trans. 1 1985, 391-398. (d) Kertesz, D. J.; Kluge, A. F. J. Org. Chem. 1988, 53, 4962-4968.

(15) Brown, H. C.; Chandrasekharan, J.; Ramachandran, P. V. J. Org. Chem. 1986, 51, 3394-3396.

(16) Corey, E. J.; Suggs, J. W. Tetrahedron Lett. 1975, 16, 2647-2650. 
(17) Übersicht: Todd, D. Org. React. 1948, 4, 378-422.

(18) Huang-Minlon J. Am. Chem. Soc. 1946, 68, 2487-2488.

(19) Christol, H.; Vanel, R. Bull. Soc. Chim. Fr. 1968, 4, 1398-1402.

(20) Cargill, R. L.; Foster, A. M. J. Org. Chem. 1970, 35, 1971-1972.

(21) Xiong, H.; Rieke, R. D. Tetrahedron Lett. 1991, 32, 5269-5272.

(22) Rieke, R. D.; Xiong, H. J. Org. Chem. 1992, 57, 6560-6565.

(23) Kakiuchi, K.; Okada, H.; Kanehisa, N.; Kai, Y.; Kurosawa, H. J. Org. Chem. 1996, 61, 2972-2979.

(24) Bak, D. A.; Brady, W. T. J. J. Org. Chem. 1979, 44, 107-110.

(25) Übersicht: Gutsche, C. D. Org. React. 1954, 8, 365-429.

(26) Fitjer, L.; Rissom, B.; Kanschik, A.; Egert, E. Tetrahedron 1994, 50, 10879-10892.

(27) Trost, B. M.; Bogdanowicz, M. J. J. Am. Chem. Soc. 1973, 95, 5298-5307.

(28) Trost, B. M.; Bogdanowicz, M. J. J. Am. Chem. Soc. 1973, 95, 5321-5334.

(29) Bogdanowicz, M. J.; Trost, B. M. Org. Synth. 1974, 54, 27-32.

(30) Trost, B. M.; Keeley, D. E.; Arndt, H. C.; Rigby, J. H.; Bogdanowicz, M. J. J. Am. Chem. Soc. 1977, 99, 3080-3087.

(31) Trost, B. M.; Keeley, D. E.; Arndt, H. C.; Bogdanowicz, M. J. J. Am. Chem. Soc. 1977, 99, 3088-3100.

(32) Crandall, J. K.; Conover, W. W. J. Org. Chem. 1978, 43, 3533-3535.

(33) Utimoto, K.; Tamura, M.; Sisido, K. Tetrahedron 1973, 29, 1169-1171.

(34) van de Heuvel, C. J. M.; Hofland, A.; van Velzen, J. C.; Steinberg, H.; de Boer, T. J. Rec. Trav. Chim. Pays-Bas 1984, 103, 233-240.

(35) Baldwin, J. E.; Höfle, G. A.; William Lever Jr., O. J. Am. Chem. Soc. 1974, 96, 71257127.

(36) Fitjer, L.; Quabeck, U. Synth. Commun. 1985, 15, 855-864.

(37) Leriverend, M. L.; Leriverend, P.; Normant, H. Compt. Rend. 1975, 280, 791-792.

(38) Leriverend, M. L.; Leriverend, P. Chem. Ber. 1976, 109, 3492-3495.

(39) Trost, B. M.; Latimer, L. H. J. Org. Chem. 1978, 43, 1031-1040.

(40) Wang, B. M.; Song, Z. L.; Fan, C. A.; Tu, Y. Q.; Chen, W. M. Synlett 2003, 14971499.

(41) Neumann, H.; Seebach, D. Chem. Ber. 1978, 111, 2785-2812.

(42) Nordvik, T.; Brinker, U. H. J. Org. Chem. 2003, 68, 9394-9399.

(43) Fitjer, L.; Steeneck, C.; Gaini-Rahimi, S.; Schroeder, U.; Justus, K.; Puder, P.; Dittmer, M.; Hassler, C.; Weiser, J.; Noltemeyer, M.; Teichert, M. J. Am. Chem. Soc. 1998, 120, 317-328.

(44) Clarembeau, M.; Cravador, A.; Dumont, W.; Hevesi, L.; Krief, A. Tetrahedron 1985, 41, 4793-4812.

(45) Krief, A. Tetrahedron 1980, 36, 2531-2540.

(46) Barton, D. H. R.; Ives, D. A. J.; Thomas, B. R. J. Chem. Soc. (London) 1955, 2056. 
(47) Johnson, C. R.; Zeller, J. R. J. Am. Chem. Soc. 1982, 104, 4021-4023.

(48) Johnson, C. R.; Zeller, J. R. Tetrahedron 1984, 40, 1225-1233.

(49) Le, N. A.; Jones Jr., M.; Bickelhaupt, F.; de Wolf, W. H. J. Am. Chem. Soc. 1989, 111, 8491-8493.

(50) Mayer, R.; Wenschuh, G.; Töpelmann, W. Chem. Ber. 1958, 91, 1616-1619.

(51) Krapcho, A. P.; Jr. Jahngen, E. G. E. J. Org. Chem. 1974, 39, 1650-1653.

(52) Fitjer, L.; Quabeck, U. Synthesis 1987, 299-300.

(53) de Boer, T. J.; Backer, H. J. Org. Synth. 1963, Coll. Vol. 4, 943-946.

(54) Taylor, E. C.; Chiang, C.; McKillop, A. Tetrahedron Lett. 1977, 1827-1830.

(55) Villieras, J.; Perriot, P.; Normant, J. F. Synthesis 1979, 968-970.

(56) Aoyama, T.; Shioiri, T. Synthesis 1988, 228-229.

(57) Prelog, V. Pure Appl. Chem. 1964, 9, 119-130.

(58) Yamago, S.; Nakamura, E. Tetrahedron 1989, 45, 3081-3088.

(59) Mundy, B. P.; Otzenberger, R. D. J. Org. Chem. 1973, 38, 2109-2110.

(60) Wohl, R. A. J. Org. Chem. 1973, 38, 3862-3864.

(61) Fitjer, L. Angew. Chem., Int. Ed. Engl. 1976, 15, 763-764.

(62) Fitjer, L. Chem. Ber. 1982, 115, 1047-1060.

(63) McMannus, S. P.; Ortiz, M.; Abramovitch, R. A. J. Org. Chem. 1981, 46, 336-342.

(64) Mousseron, M.; Jacquier, R.; Christol, H. Bull. Soc. Chim. Fr. 1957, 346.

(65) Imamoto, T.; Takiyama, N.; Nakamura, K.; Hatajima, T.; Kamiya, J. J. Am. Chem. Soc. 1989, 111, 4392-4398.

(66) Schöllkopf, U.; Hänßle, P. Liebigs Ann. Chem. 1972, 763, 208-210.

(67) Denis, J. M.; Girard, C.; Conia, J. M. Synthesis 1972, 549-551.

(68) Leriverend, M. L. Compt. Rend. 1974, 279, 755-757.

(69) Weiser, J.; Holthausen, M. C.; Fitjer, L. J. Comput. Chem. 1997, 18, 1264-1281.

(70) Allinger, N. L.; Yuh, Y. H.; Lii, J.-H. J. Am. Chem. Soc. 1989, 111, 8551-8556.

(71) Patt, S. L.; Shoolery, J. J. Magn. Res 1982, 46, 535-539.

(72) Day, A. C.; Whiting, M. C. Org. Synth. 1972; 50, 3-6.

(73) Krieger, H.; Ruotsalainen, H.; Montin, J. Chem. Ber. 1966, 99, 3715-3717.

(74) Zimmermann, D.; Ottinger, R.; Reisse, J. Org. Magn. Res. 1974, 6, 346-354.

(75) Horner, L.; Christmann, A.; Chem. Ber. 1963, 96, 388-398.

(76) Abell, P. I.; Chiao, C. J. Am. Chem. Soc. 1960, 82, 3610-3613. 


\section{DANKSAGUNG}

Meinem Lehrer, Herrn Prof. Dr. L. Fitjer, danke ich ganz herzlich für die Überlassung des interessanten Themas, die fortwährende Unterstützung, seine vielfältigen Anregungen und sofortige Beantwortung all meiner Fragen.

Für ihre Unterstützungen im Labor und im Computerraum, ihre Geduld und ihr Verständnis für meine ständig wechselhafte Laune während dieser Arbeit danke ich besonders Herrn Ralf Gerke, Herrn Dr. P. Grzeganek und Frau Uta Kaiser.

Dem ganzen Team der NMR-Abteilung danke ich ganz herzlich für die unkonventionelle Bereitstellung von Geräten und Messzeiten.

Dank an Herrn Dr. Frauendorf und Frau G. Udvarnoki für die Aufnahme der Massenspektren.

Für die Bearbeitung der Elementaranalysen danke ich Herrn F. Hambloch.

Mark danke ich für die tröstenden Worte und die Übernahme vieler privaten Aufgabe während der Erstellung dieser Arbeit.

Mein größter Dank gilt meinen Eltern für ihr Vertrauen und ihre Unterstützung während der gesamten Studienzeit. 



\section{LEBENSLAUF}

Am 30.09.1974 wurde ich, Tien Widjaja, in Surabaya, Indonesien als Tochter von Imelda Djuana und Oentoeng Setya Budi geboren. Von 1980-1986 besuchte ich die Grundschule Petra in Surabaya, von 1986-1992 das Gymnasium Petra in Surabaya, wo ich 1992 das Abitur abschloss. Von 1992-1993 besuchte ich das Studienkolleg der Universität Hannover und erwarb 1993 die deutsche Hochschulzugangsberechtigung. Im Wintersemester 1993 nahm ich das Chemiestudium an der Georg-August-Universität in Göttingen auf. Im Juni 1999 bestand ich dort die Diplomvorprüfung im Fach Chemie. Unter der Leitung von Prof. Dr. P. R. Schreiner begann ich im September 2001 in seinem Arbeitskreis in Department of Chemistry, University of Georgia in Athens, USA und im Arbeitskreis von Prof. Dr. A. de Meijere am Organisch Chemischen Institut in Göttingen, mit meiner Diplomarbeit über das Thema „Phasentransferkatalysierte Halogenierungen von Kohlenwasserstoffen mit Bicyclo[2.2.2]octan-Grundgerüst“ und legte im November 2002 die Diplomprüfung im Fach Chemie ab. Seit März 2003 arbeite ich im Arbeitskreis von Prof. Dr. L. Fitjer an meiner Dissertation. 